\title{
Dynamic Interest-Rate modelling in Incomplete Markets
}

\author{
PhD. Thesis
}

Jesús Pérez Colino

Departamento de Estadística

Thesis Supervisors:

Francisco Javier Nogales

Universidad Carlos III de Madrid

Winfried Stute

Giessen University

\section{Universidad Carlos III de Madrid}




\title{
Dynamic Interest-Rate modelling in Incomplete Markets by Jesús Pérez Colino
}

\author{
Submitted to the Departamento de Estadística \\ on November, 2008, in partial fulfillment of the \\ requirements for the degree of \\ Doctor of Philosophy
}

\begin{abstract}
In the first chapter, a new kind of additive process is proposed. Our main goal is to define, characterize and prove the existence of the LIBOR additive process as a new stochastic process. This process will be defined as a piecewise stationary process with independent increments, continuous in probability but with discontinuous trajectories, and having "càdlàg" sample paths. The proposed process is specifically designed to derive interest-rates modelling because it allows us to introduce a jump-term structure as an increasing sequence of Lévy measures. In this paper we characterize this process as a Markovian process with an infinitely divisible, selfsimilar, stable and self-decomposable distribution. Also, we prove that the Lévy-Khintchine characteristic function and Lévy-Itô decomposition apply to this process. Additionally we develop a basic framework for density transformations. Finally, we show some examples of LIBOR additive processes.

A no-arbitrage framework to model interest rates with credit risk, based on the LIBOR additive process, and an approach to price corporate bonds in incomplete markets, is presented in the second chapter. We derive the no-arbitrage conditions under different conditions of recovery, and we obtain new expressions in order to estimate the probabilities of default under risk-neutral measure. Additionally, we study both the approximation of a continuous-time model by a sequence of discrete-time models with credit risk, and the convergence of price processes (in terms of the triplets) under a framework that allows the practitioner a multiple set of models (semimartingale) and credit conditions (migration and default).

Finally, in the third chapter, we introduce a d-dimensional LIBOR additive process to model the forward LIBOR market model with different credit ratings. Additionally, we expose the risk-neutral and forward-neutral dynamic of forward LIBOR rates. Additionally, we introduce a new calibration methodology for the LIBOR market model driven by LIBOR additive processes. The calibration of the continuous part is based on a semidefinite programming (convex) problem and the calibration of the Lévy measure is proposed using a non-parametric (non linear least-square with a regularization term) calibration.
\end{abstract}

Thesis Supervisor: Francisco Javier Nogales

Title: Associate Professor (UC3M)

Thesis Supervisor: Winfried Stute

Title: Professor (Giessen University) 


\section{Contents}

1 New stochastic processes to model interest rates: LIBOR additive processes $\quad 6$

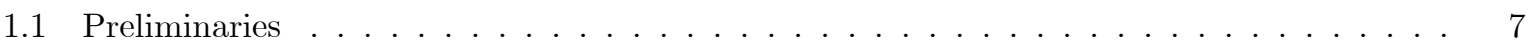

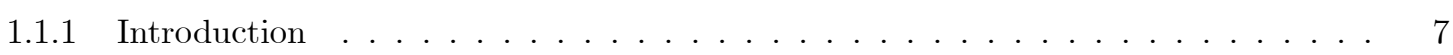

1.1.2 Some frequently used notation and terminology $\ldots \ldots \ldots \ldots \ldots$

1.1.3 Basics about Lévy Processes $\ldots \ldots \ldots \ldots \ldots \ldots$

1.2 Definition and Properties of LIBOR Additive Processes . . . . . . . . . . . . . . 14

1.2 .1 The LIBOR additive process $\ldots \ldots \ldots \ldots \ldots \ldots$

1.2 .2 Properties of LIBOR additive processes $\ldots \ldots \ldots \ldots \ldots \ldots$

1.3 Existence of LIBOR additive processes $\ldots \ldots \ldots \ldots \ldots \ldots \ldots \ldots \ldots$

1.4 Characterization of LIBOR additive processes $\ldots \ldots \ldots \ldots \ldots \ldots \ldots \ldots$

1.5 Infinitesimal generators of LIBOR additive processes $\ldots \ldots \ldots \ldots \ldots \ldots$

1.5.1 Strongly continuous contraction semigroup and infinitesimal generator . . . . . 25

1.5.2 Infinitesimal generators of LIBOR additive processes $\ldots \ldots \ldots \ldots \ldots$

1.6 The Lévy-Itô decomposition of LIBOR additive processes . . . . . . . . . . . . . . . 28

1.7 Applications to sample-function properties: continuity, jumping times and increasingness . 31

1.8 Density Transformations of LIBOR additive processes $\ldots \ldots \ldots \ldots \ldots$

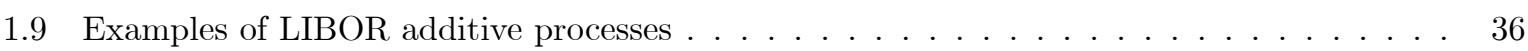

1.9.1 The non-homogeneous Poisson Process . . . . . . . . . . . . . . . 36

1.9.2 The non-homogeneous Compound Poisson Process . . . . . . . . . . . . . 36

1.9.3 The non-homogeneous Gamma Process . . . . . . . . . . . . . . . 38

1.9.4 The non-homogeneous Inverse Gaussian Process . . . . . . . . . . . . . . . 38 
1.9.5 The non-homogeneous Generalized Inverse Gaussian Process ........ 39

1.9.6 The non-homogeneous $\alpha$-Stable Process . . . . . . . . . . . . . . . . . . . . . . 40

1.9.7 The non-homogeneous Tempered Stable Process . . . . . . . . . . . . . . . . 41

1.9.8 The non-homogeneous Variance Gamma Process . . . . . . . . . . . . . . . . . . . 42

1.9.9 The non-homogeneous Normal Inverse Gaussian Process . . . . . . . . . . . . . . . 44

1.9.10 The non-homogeneous CGMY Process . . . . . . . . . . . . . . . . . . . . 45

1.9.11 The non-homogeneous Meixner Process . . . . . . . . . . . . . . . . . 47

1.9.12 The non-homogeneous Generalized Hyperbolic Process . . . . . . . . . . . . . . . . 47

\section{Reaction-Additive systems to modelling Corporate Bonds: no-arbitrage and weak convergence conditions $\quad 449$}

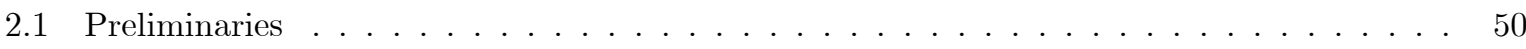

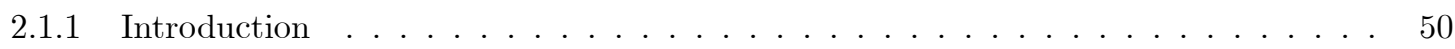

2.1.2 Basic Assumptions for the risk-free Interest Rates model. . . . . . . . . . . . . . . 51

2.1.3 Basic Assumptions for the Credit Risk Model . . . . . . . . . . . . . . . . 52

2.2 The building-blocks for Interest-Rate modelling . . . . . . . . . . . . . . . . . . . . 58

2.2.1 Risk-free Bond Market Structure . . . . . . . . . . . . . . . . . . . 58

2.2.2 Conditional Corporate-Bond market structure . . . . . . . . . . . . . . . . 61

2.2.3 Corporate-Bond Market Structure with Credit Migration and Default . . . . . . 63

2.3 Absence of arbitrage and dynamics under a martingale measure . . . . . . . . . . . 68

2.3.1 Absence of arbitrage condition in a corporate-bond market with fractional recovery of market value . . . . . . . . . . . . . . . . . . . . . . 69

2.3.2 Absence of arbitrage in a corporate-bond market with fractional recovery of treasury 72

2.3.3 Absence of arbitrage in a corporate-bond market with fractional recovery of par value 73

2.4 Corporate-Bond valuation in a Reaction-Additive system . . . . . . . . . . . . . . 75

2.4.1 Classical and Viscosity solutions for reaction-additive systems under local regularity 76

2.4.2 Markov property and uniqueness . . . . . . . . . . . . . . . 78

2.4.3 Sample-paths in the Skorohod space $D_{\left[0, T^{\star}\right]}\left(\mathbb{R}^{d}, \mathcal{K}\right) \ldots \ldots \ldots$. . . . . . . 79

2.5 Uniform Weak Convergence in the Reaction-Additive system . . . . . . . . . . . . . . 84

2.5.1 Introduction to Weak Convergence . . . . . . . . . . . . . . . . 85 
2.5.2 Relative compactness in the space $D_{\left[0, T^{*}\right]}\left(\mathbb{R}^{d}, \mathcal{K}\right) \ldots \ldots \ldots \ldots \ldots$

2.5.3 Weak Convergence in $D_{\left[0, T^{\star}\right]}\left(\mathbb{R}^{d}, \mathcal{K}\right) \ldots \ldots \ldots \ldots \ldots$

2.5.4 Previsibility conditions for weak convergence of $G^{C}$ in the space $D_{\left[0, T^{\star}\right]}\left(\mathbb{R}^{d}, \mathcal{K}\right) \ldots 97$

3 LIBOR Market Model driven by LIBOR additive processes: Pricing and Calibration113

3.1 Preliminaries . . . . . . . . . . . . . . . . . . . . . . . . . 114

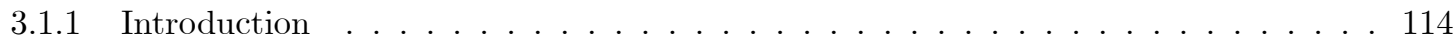

3.1.2 Basic assumption for the LIBOR additive model with credit transitions . . . . . 115

3.1.3 The LIBOR market model: a quick review . . . . . . . . . . . . . . . . . . . 118

3.2 The LIBOR Market Model driven by a LIBOR additive process . . . . . . . . . . . . . . 125

3.2.1 General framework: an introduction to the extended LMM . . . . . . . . . . . 125

3.2.2 No-arbitrage conditions under the risk-neutral measure f . . . . . . . . . . 128

3.3 LIBOR additive model calibration to swaptions markets $\ldots \ldots \ldots \ldots \ldots \ldots$

3.3.1 An Introduction to the two-steps calibration for the LIBOR additive process . . 134

3.3.2 First-step: semidefinite programming to calibrate the continuous Market model . . 136

3.3.3 Second-step: the jump-part calibration . . . . . . . . . . . . . . . . . . . . . . 149

3.3 .4 Numerical Performance . . . . . . . . . . . . . . . . . . . . . . . . . 153 
Chapter 1

New stochastic processes to model interest rates: LIBOR additive processes 


\subsection{Preliminaries}

\subsubsection{Introduction}

The story of modelling financial markets with stochastic processes began in 1900 with the study of Louis Bachelier (1900). He modelled stocks as a Brownian motion with drift. However, the model had many imperfections, including, for example, negative stock prices. It was 65 years before another, more appropriate, model was suggested by Samuelson (1965): geometric Brownian motion. Eight years later Black and Scholes (1973) and Merton (1973) demonstrated how to price European options based on the geometric Brownian motion. This stock-price model is now called Black-Scholes model, for which Scholes and Merton received the Nobel Prize for Economics in 1997.

However, it has become clear that this option-pricing model is inconsistent with option data. Implied volatility models can do better, but fundamentally, these consist of the wrong building blocks. To improve on the performance of the Black-Scholes model, Lévy models were proposed in the late 1980s and in the early 1990s, when there was some need for models taking into account of different stylized features of the market.

On the other hand, traditionally, interest rates models for Treasury bonds or Corporate bonds, in the literature, are mainly models based on Brownian motion although it is known that real-life financial markets provide a different structural and statistical behavior than that implied by these models. Some of these interest rates models have been created thinking in Black-Scholes framework, but they found a great number of inconveniences. Also in this field, Lévy processes are proposed as an appropriate tool to increase the accuracy of interest rates models. However, the nature of random sources in bond markets is different from equity markets:

1. Modelling a term structure is completely different from modelling a simple equity. There exists a collection of 'correlated' bonds that generate a 'multifactorial' term structure. In other words, we have a stochastic surface that is strongly linked to some no arbitrage conditions, completely different to the no-arbitrage conditions that appear in equity markets.

2. Derivative markets for interest rates (caps/floors and swaptions markets) quote differently 'atthe-money' volatilities according to the different maturities inside of the term structure (forward volatilities). While Brownian or Lévy processes offer nice features in terms of analytical tractability, the constraints of independence and stationarity of their increments prove to be very restrictive for this market.

3. Also, these derivative markets quote, for each maturity, a different volatility for every strike ('inthe-money' or 'out-of-the-money' options). Lévy models allow for calibration to implied volatility patterns for a single maturity but fail to reproduce option prices correctly over a range of different maturities. In addition, the existence of this term structure of 'volatility smiles' have a huge impact in order to price not only plain vanilla interest rate options but also exotic options.

In the First Chapter ${ }^{1}$ of the present thesis, we present a stochastic process that is specifically designed to represent the random sources that appear in this market, and we develop some basic tools that any interest rate model needs in order to reproduce the risk neutral dynamics. This Chapter is organized as follows.

\footnotetext{
${ }^{1}$ This work has been developed under the direction of Javier Nogales and Winfried Stute. I am extremely grateful to them. I also benefited greatly in my work from discussions with C. Rogers, W. Runggaldier, Rama Cont, David Nualart and Henryk Gzyl. Comments and suggestions are welcome, all errors are my own.

Contact email: jpcolino@gmail.com
} 
- In Section 2 we introduce the stochastic processes of interest together with their main distributional properties (infinite divisibility, self-similarity, stability and self-decomposability).

- Section 3 introduces the characterization of these processes, using or adapting the well-known Lévy-Khintchine formula to this framework.

- In Section 4 we propose some limit theorems that will be developed in detail in Chapter 2 of this thesis.

- Section 5 is mainly devoted to the existence of this new process, mainly under the framework for Markovian processes.

- In Section 6 we adapt the Lévy-Itô decomposition, and we deduce some interesting applications that appear in Section $\%$.

- Probably, Section 8 is the most important section in order to build models using this process. Here, we expose the two possibilities that any financial engineering has in order to find the risk-neutral measures: time-changes (subordination) and changes of measure.

- Finally Section 9 is devoted to expose different examples of non-homogeneous processes that can be used as a LIBOR additive process.

\subsubsection{Some frequently used notation and terminology}

A probability space $(\Omega, \mathcal{F}, \mathbb{P})$ is a triplet of a set $\Omega$, a family $\mathcal{F}$ of subsets of $\Omega$, and a mapping $\mathbb{P}$ from $\mathcal{F}$ into $\mathbb{R}^{+}$satisfying the following conditions:

(1) $\Omega \in \mathcal{F}, \emptyset \in \mathcal{F}(\emptyset$ is the empty set $)$

(2) If $A_{n} \in \mathcal{F}$ for $n=1,2, \ldots$, then $\bigcup_{n=1}^{\infty} A_{n}$ and $\bigcap_{n=1}^{\infty} A_{n}$ are in $\mathcal{F}$

(3) If $A \in \mathcal{F}$ then $A^{c} \in \mathcal{F}\left(A^{c}\right.$ is $\Omega \backslash A$, the complement of $\left.A\right)$

(4) $0 \leq \mathbb{P}[A] \leq 1, \mathbb{P}[\Omega]=1$ and $\mathbb{P}[\emptyset]=0$

(5) If $A_{n} \in \mathcal{F}$ for $n=1,2, \ldots$ and they are disjoint (that is $A_{n} \cap A_{m}=\emptyset$ for $n \neq m$ ) then

$$
\mathbb{P}\left[\bigcup_{n=1}^{\infty} A_{n}\right]=\sum_{n=1}^{\infty} \mathbb{P}\left[A_{n}\right]
$$

In terminology of measure theory, a probability space is a measure space with total measure 1 . In general, if $\mathcal{F}$ is a family of subsets of $\Omega$ satisfying (1), (2), and (3) then $\mathcal{F}$ is called a $\sigma$-algebra on $\Omega$. The pair $(\Omega, \mathcal{F})$ is called a measurable space. Very often in this work, we have $\mathcal{F}=\mathcal{F}_{\infty}=\vee_{t \geq 0} \mathcal{F}_{t}$ where $\left(\mathcal{F}_{t}\right)_{t>0}$ is a non-decreasing and right-continuous family of $\sigma$-algebras (in other words, $\mathcal{F}_{s} \subset \mathcal{F}_{t}$ for $s \leq t$, and $\left.\overline{\mathcal{F}}_{t}=\cap_{s>t} \mathcal{F}_{s}\right)$. In such a situation a filtered probability space $(\Omega, \mathcal{F}, \mathbb{P})$ is called a stochastic basis . During the whole work we denote by $\mathcal{B}\left(\mathbb{R}^{d}\right)$ the collection of all Borel subsets of $\mathbb{R}^{d}$, called the Borel $\sigma$-algebra. It is the $\sigma$-algebra generated by the open sets in $\mathbb{R}^{d}$ (that is, the smallest $\sigma$-algebra that contains all open sets in $\left.\mathbb{R}^{d}\right)$. A real-valued function $f(x)$ on $\mathbb{R}^{d}$ is called measurable if it is $\mathcal{B}\left(\mathbb{R}^{d}\right)$ measurable.

A mapping $X$ from $\Omega$ into $\mathbb{R}^{d}$ is an $\mathbb{R}^{d}$-valued random variable (or random variable on $\mathbb{R}^{d}$ ) if it is $\mathcal{F}$-measurable, that is $\{\omega: X(\omega) \in B\}$ is in $\mathcal{F}$ for each $B \in \mathcal{B}\left(\mathbb{R}^{d}\right)$. The distribution of an $\mathbb{R}^{d}$-valued random variable $X$ is denoted by $\mu=\mathbb{P}_{X}$ or $\mathcal{L}(X)$. Furthermore, $\hat{\mu}(z)$ is the characteristic function of a distribution $\mu$ defined as

$$
\hat{\mu}(z)=\int_{\mathbb{R}^{d}} \exp \{i\langle z, x\rangle\} \mu(d x) \quad, z \in \mathbb{R}^{d}
$$


and $\psi_{\mu}(z)$ is the cumulant of $\mu$, that is, the continuous function with $\psi_{\mu}(0)=0$ such that $\hat{\mu}(z)=$ $\exp \left(\psi_{\mu}(z)\right)$. The characteristic function of the distribution $\mathbb{P}_{X}$ of a random variable $X$ on $\mathbb{R}^{d}$ is denoted by $\hat{\mathbb{P}}_{X}(z)$ and given as

$$
\begin{aligned}
\hat{\mathbb{P}}_{X}(z) & =\int_{\mathbb{R}^{d}} \exp \{i\langle z, x\rangle\} \mathbb{P}_{X}(d x) \\
& =\mathbb{E}[\exp \{i\langle z, X\rangle\}], \quad \text { with } z \in \mathbb{R}^{d}
\end{aligned}
$$

A sequence of probability measures $\mu_{n}, n=1,2, \ldots$ converges to a probability measure $\mu$, written as

$$
\mu_{n} \rightarrow \mu \quad \text { as } n \rightarrow \infty,
$$

if for every bounded continuous function $f$

$$
\int_{\mathbb{R}^{d}} f(x) \mu_{n}(d x) \rightarrow \int_{\mathbb{R}^{d}} f(x) \mu(d x) \quad \text { as } n \rightarrow \infty
$$

When $\mu$ and $\mu_{n}$ are bounded measures, the convergence $\mu_{n} \rightarrow \mu$ is defined in the same way. When $\left\{\mu_{t}\right\}$ are probability measures, we say that

$$
\mu_{s} \rightarrow \mu_{t} \quad \text { as } s \rightarrow t
$$

if for every bounded continuous function $f(x)$

$$
\int_{\mathbb{R}^{d}} f(x) \mu_{s}(d x) \rightarrow \int_{\mathbb{R}^{d}} f(x) \mu_{t}(d x) \quad \text { as } s \rightarrow t .
$$

This is equivalent to saying that $\mu_{s} \rightarrow \mu_{t}$ for every sequence $s$ that tends to $t$.

We say that $B$ is a $\mu$-continuity set if the boundary of $B$ has $\mu$-measure 0 . The convergence $\mu_{n} \rightarrow \mu$ is equivalent to the condition that $\mu_{n}(B) \rightarrow \mu(B)$ for every $\mu$-continuity set $B \in \mathcal{B}\left(\mathbb{R}^{d}\right)$. by

The convolution $\mu$ of two distributions $\mu_{1}$ and $\mu_{2}$ on $\mathbb{R}^{d}$ denoted by $\mu=\mu_{1} * \mu_{2}$ is a distribution defined

$$
\mu(B)=\iint_{\mathbb{R}^{d} \times \mathbb{R}^{d}} 1_{B}(x+y) \mu_{1}(d x) \mu_{2}(d y)
$$

The convolution operation is commutative and associative. If $X_{1}$ and $X_{2}$ are independent random variables on $\mathbb{R}^{d}$ with distributions $\mu_{1}$ and $\mu_{2}$, respectively, then $X_{1}+X_{2}$ has distribution $\mu_{1} * \mu_{2}$. The $n$-fold convolution of $\mu$ is denoted by $\mu^{n *}$. A probability measure $\mu$ on $\mathbb{R}^{d}$ is infinitely divisible if, for any positive integer $n$, there is a probability measure $\mu_{(n)}$ on $\mathbb{R}^{d}$ such that $\mu=\mu_{(n)}^{n *}$.

A family $\left\{X_{t}: t \geq 0\right\}$ of random variables on $\mathbb{R}^{d}$ with parameter $t \in[0, \infty)$ defined on a common probability space is called a stochastic process. For any fixed $0 \leq t_{1}<t_{2}<\ldots<t_{n}$,

$$
\mathbb{P}\left[X\left(t_{1}\right) \in B_{1}, \ldots, X\left(t_{n}\right) \in B_{n}\right]
$$

determines a probability measure on $\mathcal{B}\left(\left(\mathbb{R}^{d}\right)^{n}\right)$. The family of probability measures over all choices of $n$ and $t_{1}, t_{2}, \ldots, t_{n}$ is called the system of finite dimensional distributions of $\left\{X_{t}\right\}$. Given two stochastic processes $\left\{X_{t}, t \geq 0\right\}$ and $\left\{Y_{t}, t \geq 0\right\},\left\{X_{t}\right\} \stackrel{d}{=}\left\{Y_{t}\right\}$ means that $X$ and $Y$ are identical in law or have a common system of finite-dimensional marginals. A stochastic process $\left\{Y_{t}\right\}$ is called a modification of a stochastic process $\left\{X_{t}\right\}$ if $\mathbb{P}\left[X_{t}=Y_{t}\right]=1$ for $t \in[0, \infty)$.

We say that $X$ is a semimartingale if it is an adapted process $\left(\mathcal{F}_{t}\right.$-measurable for every $\left.t \in[0, \infty)\right)$ 
such that $X_{t}=X_{0}+M_{t}+V_{t}$ for each $t \geq 0$, where $X_{0}$ is finite-valued and $\mathcal{F}_{0}$-measurable, $\left\{M_{t}, t \geq 0\right\}$ is a local martingale (for a localizing sequence of stopping times $\left(T_{n}\right), M_{n}$ is an adapted process, integrable for each $n \in \mathbb{N}_{+}$and $M_{n-1}=\mathbb{E}\left(M_{n} / \mathcal{F}_{n-1}\right)<\infty$ a.s $)$ and where $\left\{V_{t}, t \geq 0\right\}$ is an adapted process of finite variation.

Sometimes it will be necessary to work on the path space of "càdlàg" (i.e. right-continuous with left limits) semimartingales. For $I=\left[0, T^{\star}\right] \subseteq[0, \infty)$ with $T^{\star}>0$, we denote by $\mathbb{D}=\mathbb{D}\left(\mathbb{R}^{d}, I\right)$ the Skorohod space of all càdlàg functions $\alpha: I \rightarrow \mathbb{R}^{d}$. For $I=\mathbb{R}_{+}=[0, \infty)$ we denote by $\mathcal{D}_{t}^{0}\left(\mathbb{R}^{d}\right)$ the $\sigma$-field generated by all mappings $\alpha \mapsto \alpha(s)$ for $s \leq t$, and $\mathcal{D}_{t}\left(\mathbb{R}^{d}\right)=\cap_{s>t} \mathcal{D}_{s}^{0}\left(\mathbb{R}^{d}\right)$. If $X$ is a semimartingale on $(\Omega, \mathcal{F}, \mathbb{P})$ such that $X \in \mathbb{D}\left(\mathbb{R}^{d}, I\right)$ we denote by $X(t)$ the value of $X$ at time $t$, and by $X(t-)$ its left-hand limit at time $t$ (with $X(0-)=X(0)$ by convention) and $\triangle X(t)=X(t)-X(t-)$.

We will use, during the whole work, the definitions as outlined in Sato (1999) of infinite divisibility of processes, self-decomposability (of distributions), self-similarity and stability of processes, Lévy processes, additive processes, increasing or decreasing processes. We use ':=' to mean 'is defined to be equal to'. In particular we set $\mathbb{R}_{+}:=[0, \infty), \mathbb{R}_{+}^{d}:=[0, \infty)^{d}, \mathbb{Z}_{+}:=\mathbb{Z} \cap \mathbb{R}_{+}, \mathbb{Q}_{+}:=\mathbb{Q} \cap \mathbb{R}_{+}$. Elements of $\mathbb{R}^{d}$ are column vectors. If $f$ and $g$ are real numbers or real-valued functions, we define $f \vee g:=\max (f, g)$, $f \wedge g:=\min (f, g), f^{+}:=f \vee 0, f^{-}:=(-f) \vee 0$. In particular, we have $f=f^{+}-f^{-}$and $|f|=f^{+}+f^{-}$. For $x=\left(x_{j}\right)_{1 \leq j \leq d}$ and $y=\left(y_{j}\right)_{1 \leq j \leq d}$ in $\mathbb{C}^{d}$ we write $\langle x, y\rangle=\sum_{j=1}^{d} x_{j} y_{j}$.

\subsubsection{Basics about Lévy Processes}

The aim of this preliminary section is to give a brief introduction to the theory of Lévy processes. Inclusion of this material is justified not only because Brownian motion or Poisson processes are Lévy processes, but also because an additive process will be defined later as a nonhomogeneous Lévy process. Additionally, Lévy processes also provide one of the most important examples of Markov processes and semimartingales.

This preliminary section is an attempt to gather some basic and typical results to describe several main directions of this chapter. It is not intended to give a systematic presentation of the most important results or to explain how to prove them; for these purposes one would need many more pages. A more comprehensive picture of the present knowledge can be obtained from the two books Bertoin (1996) and Sato (1999).

Definition 1 An $\mathbb{R}^{d}$-valued stochastic process $X=\left\{X_{t}: t \geq 0\right\}$ is a family of $\mathbb{R}^{d}$-valued random variables $X_{t}(\omega)$ with parameter $t \in[0, \infty)$ defined on a probability space $(\Omega, \mathcal{F}, \mathbb{P})$.

Definition 2 An $\mathbb{R}^{d}$-valued stochastic process $X=\left\{X_{t}: t \geq 0\right\}$ is called a Lévy process in law on $\mathbb{R}^{d}$ or d-dimensional Lévy process, if the following four properties are satisfied:

(L1) $X$ starts at the origin, $X_{0}=0$ a.s. (almost surely)

(L2) $X$ has independent increments, that is, for any $n \geq 1$ and $0 \leq t_{0}<t_{1}<\ldots<t_{n}$, the random variables $X_{t_{0}}, X_{t_{1}}-X_{t_{0}}, X_{t_{2}}-X_{t_{1}}, \ldots, X_{t_{n}}-X_{t_{n-1}}$ are independent.

(L3) $X$ is stochastically continuous, that is, for any $\varepsilon>0, P\left[\left|X_{t+s}-X_{t}\right|>\varepsilon\right] \rightarrow 0$ as $s \rightarrow 0$, for all $t \geq 0$.

(L4) $X$ is time homogeneous (or stationary), that is, the distribution of $\left\{X_{s+t}-X_{s}: t \geq 0\right\}$ does not depend on $s$ 
Notice that if $X$ is a stochastically continuous process with independent and stationary increments (a Lévy process in law), there exists a càdlàg version of $X$ with the same properties called Lévy process (cf. He, Wang and Yan (1992), Theorem 2.68). Therefore a Lévy process can be defined as a $d$ dimensional stochastic process starting in 0 with càdlàg paths and independent and stationary increments under $\mathbb{P}$ (if there is no ambiguity about the measure involved) (cf. Bertoin (1996)).

Proposition 3 If $X$ is a Lévy process, then $X(t)$ is infinitely divisible for each $t \geq 0$.

Proof. To see this, let $t_{i}=i t / m$ with $i=0,1, \ldots, m$ and some $t \geq 0$. Let $\mu=\mathbb{P}_{X_{t}}$ and $\mu_{m}=\mathbb{P}_{X_{t_{i}}-X_{t_{i-1}}}^{(m)}$ which is independent of $i$ by temporal homogeneity. Then $\mu=\mu_{(m)}^{m *}$, since

$$
X_{t}=\left(X_{t_{1}}^{(m)}-X_{t_{0}}^{(m)}\right)+\ldots+\left(X_{t_{m}}^{(m)}-X_{t_{m-1}}^{(m)}\right)
$$

is a sum of $m$ independent identically distributed random variables.

Recall that the characteristic function of a distribution $\mu$ on $\mathbb{R}^{d}$ is defined by $\hat{\mu}(z)=\int_{\mathbb{R}^{d}} e^{i\langle z, x\rangle} \mu(d x)$, $z \in \mathbb{R}^{d}$. Also remember that here $\langle z, x\rangle=\sum_{j=1}^{d} z_{j} x_{j}$, the Euclidean inner product of $z=\left(z_{j}\right)$ and $x=\left(x_{j}\right)$ in $\mathbb{R}^{d}$. Thus $|x|=\langle x, x\rangle^{1 / 2}$.

Proposition 4 If $X$ is a Lévy process, then $\hat{\mu}_{X_{t}}(z)=\exp \{t \cdot \Psi(z)\}$ for each $z \in \mathbb{R}^{d}, t \geq 0$ and where $\Psi(z)$ is the Lévy exponent.

Proof. For the sake of clarity, define $\hat{\mu}_{z}(t)=\hat{\mu}_{X_{t}}(z)$. Then for all $s \geq 0$

$$
\begin{aligned}
\hat{\mu}_{z}(t+s) & =\mathbb{E}\left(e^{i\left\langle z, X_{t+s}\right\rangle}\right) \\
& =\mathbb{E}\left(e^{i\left\langle z, X_{t+s}-X_{s}\right\rangle} e^{i\left\langle z, X_{s}\right\rangle}\right) \\
& =\mathbb{E}\left(e^{i\left\langle z, X_{t+s}-X_{s}\right\rangle}\right) \mathbb{E}\left(e^{i\left\langle z, X_{s}\right\rangle}\right) \\
& =\hat{\mu}_{z}(t) \hat{\mu}_{z}(s)
\end{aligned}
$$

Notice that using ( $L 1)$ in Definition 2 (about Lévy processes) we have $\hat{\mu}_{z}(0)=1$ and by $(L 3)$ the map $t \rightarrow \hat{\mu}_{z}(t)$ is continuous.

However the unique solution for $\hat{\mu}_{z}(t+s)=\hat{\mu}_{z}(t) \hat{\mu}_{z}(s)$ and $\hat{\mu}_{z}(0)=1$ is given by $\hat{\mu}_{z}(t)=\exp \{t \cdot \Psi(z)\}$ where $\Psi: \mathbb{R}^{d} \rightarrow \mathbb{C}$ (see e.g. Bingham et al. (1987) pp.4-6). Notice that $X(1)$ is infinitely divisible and $\Psi$ is the Lévy exponent. The result follows.

The following three theorems are fundamental. For their proofs see the monographs Doob (1953), Loève (1955), Breiman (1968), Gihman and Skorohod (1975), Kallenberg (1997) or Sato (1999).

Theorem 5 If $\mu$ is an infinitely divisible distribution on $\mathbb{R}^{d}$, then there exists, uniquely in law, a Lévy process in law $\left\{X_{t}\right\}$ such that $\mathcal{L}\left(X_{1}\right)=\mu$.

Theorem 6 If $\left\{X_{t}\right\}$ is a Lévy process in law on $\mathbb{R}^{d}$, then there is a Lévy process $\left\{X_{t}^{\prime}\right\}$ on $\mathbb{R}^{d}$ such that $\left\{X_{t}^{\prime}\right\}$ is a modification of $\left\{X_{t}\right\}$, that is $X_{t}^{\prime}=X_{t}$ a.s. for every $t \geq 0$.

Theorem 7 (Lévy-Khintchine representation) If $\mu$ is infinitely divisible, then

$$
\hat{\mu}(z)=\exp \left[i\langle\gamma, z\rangle-\frac{1}{2}\langle z, A z\rangle+\int_{\mathbb{R}^{d}}\left(e^{i\langle z, x\rangle}-1-i\langle z, x\rangle 1_{\{|x| \leq 1\}}(x)\right) v(d x)\right]
$$


where $A$ is a symmetric nonnegative-definite $d \times d$ matrix, $\gamma \in \mathbb{R}^{d}$, $v$ is a measure on $\mathbb{R}^{d}$ satisfying $v(\{0\})=0$ and $\int_{\mathbb{R}^{d}}\left(|x|^{2} \wedge 1\right) v(d x)<\infty$.

The representation (1.1) by $A, \gamma$ and $v$ is unique. Conversely, for any choice of $A, \gamma$ and $v$ satisfying the conditions above, there exists an infinitely divisible distribution $\mu$ having characteristic function (1.1).

It follows that the Lévy process $\left\{X_{t}\right\}$ corresponding to $\mu$, by Proposition 4 and Theorem 5 , has characteristic function

$$
\mathbb{E}\left[e^{i\left\langle z, X_{t}\right\rangle}\right]=\exp \left[t \cdot\left(i\langle\gamma, z\rangle-\frac{1}{2}\langle z, A z\rangle+\int_{\mathbb{R}^{d}}\left(e^{i\langle z, x\rangle}-1-i\langle z, x\rangle 1_{\{|x| \leq 1\}}(x)\right) v(d x)\right)\right]
$$

Notice that we can define $\hat{\mu}_{t}(z)=\exp \{t \cdot \Psi(z)\}$ for each $t \geq 0$, where $\Psi(z)$ is the Lévy exponent. We call $(\gamma, A, v)$ the generating triplet, $A$ the Gaussian covariance matrix, and $v$ the Lévy measure of $\mu$. However, $\gamma$ does not have any intrinsic meaning, since its value depends on the choice of $i\langle z, x\rangle 1_{\{|x| \leq 1\}}(x)$ of the integrand in (1.1) as a term to make it $v$-integrable.

If $v=0$ then $\mu$ is Gaussian. If $A=0$ then we say that $\mu$ is purely non-Gaussian. If $d=1$ then $A$ is a nonnegative real number called Gaussian variance. If $v$ satisfies $\int_{|x| \leq 1}|x| v(d x)<\infty$ then (1.1) may be written as

$$
\hat{\mu}(z)=\exp \left[i\left\langle\gamma_{0}, z\right\rangle-\frac{1}{2}\langle z, A z\rangle+\int_{\mathbb{R}^{d}}\left(e^{i\langle z, x\rangle}-1\right) v(d x)\right]
$$

with some $\gamma_{0} \in \mathbb{R}^{d}$. This $\gamma_{0}$ is called the drift. If $v$ satisfies $\int_{|x|>1}|x| v(d x)<\infty$ then (1.1) can be written as

$$
\hat{\mu}(z)=\exp \left[i\left\langle\gamma_{1}, z\right\rangle-\frac{1}{2}\langle z, A z\rangle+\int_{\mathbb{R}^{d}}\left(e^{i\langle z, x\rangle}-1-i\langle z, x\rangle 1_{\{|x| \leq 1\}}(x)\right) v(d x)\right]
$$

with some $\gamma_{1} \in \mathbb{R}^{d}$, called the center.

Brownian motion is a Lévy process with $A=$ identity matrix, $v=0$ and $\gamma=0$, and a Poisson process with intensity $\lambda>0$ is a Lévy process on $\mathbb{R}$ with $A=0, \gamma=0$ and $v=\lambda \delta_{1}$, where we denote by $\delta_{a}$ the distribution concentrated at $a$. A Lévy process on $\mathbb{R}^{d}$ with $A=0, \gamma=0$ and $v\left(\mathbb{R}^{d}\right)<\infty$ is called compound Poisson process. The $\Gamma$-process with parameter $q>0$ is a Lévy process on $\mathbb{R}$ corresponding to the exponential distribution $\mu$ with mean $1 / q$; that is $A=0, \gamma=0$ and $v(d x)=$ $1_{(0, \infty)}(x) x^{-1} e^{-q x} d x$. This $\mu$ is not a compound Poisson distribution, because $v$ has total mass $\infty$.

Any Lévy process $\left\{X_{t}\right\}$ is a Markov process and allows for a càdlàg version. Let us define two other processes: $X_{-}=\left(X_{t-}\right)_{t \in \mathbb{R}_{+}}$and $\Delta X=\left(X_{t}-X_{t-}\right)_{t \in \mathbb{R}_{+}}$.

Theorem 8 If $X$ is a Lévy process, then for fixed $t>0, \Delta X_{t}=0$ (a.s).

Proof. Let $\left(t_{(m)}, m \in \mathbb{N}_{+}\right)$be a sequence in $\mathbb{R}_{+}$with $t_{(m)} \uparrow t$ as $m \rightarrow \infty$. Then since $X$ has càdlà paths, $\lim _{m \rightarrow \infty} X\left(t_{(m)}\right)=X\left(t_{-}\right)$. However by stochastic continuity the sequence $\left(X\left(t_{(m)}\right), m \in \mathbb{N}_{+}\right)$ converges in probability to $X(t)$ and so has a subsequence that converges almost surely to $X(t)$. The result follows by uniqueness of limits.

Notice that Theorem 8 is equivalent to the fact that any process with càdlàg paths and stationary and 
independent increments has no fixed times of discontinuity ${ }^{2}$ (cf. Jacod and Shiryaev (1987), II.4.3).

The probabilistic meaning of the Lévy-Khintchine representation is explained by the following result.

Theorem 9 (Lévy-Itô decomposition of sample functions) Let $\left\{X_{t}\right\}$ be a Lévy process on $\mathbb{R}^{d}$ with the characteristic triplet $(\gamma, A, v)$. For any $G \in B_{(0, \infty) \times \mathbb{R}^{d}}$ let $J(G)=J(G, \omega)$ be the number of jumps at time $s$ with height $X_{s}(\omega)-X_{s-}(\omega)$ such that $\left(s, X_{s}(\omega)-X_{s-}(\omega)\right) \in G$. Then $J(G)$ has a Poisson distribution with mean $\mu(G)$. If $G_{1}, \ldots, G_{n}$ are disjoint, then $J\left(G_{1}\right), \ldots, J\left(G_{n}\right)$ are independent. We can define a.s.,

$$
\begin{aligned}
X_{t}^{1}(\omega)= & \lim _{\varepsilon \downarrow 0} \int_{(0, t] \times\{\varepsilon<|x| \leq 1\}}\{x J(d(s, x), \omega)-x \mu(d(s, x))\} \\
& +\int_{(0, t] \times\{|x|>1\}} x J(d(s, x), \omega)
\end{aligned}
$$

where the convergence on the right-hand side is uniform in $t$ in any finite time interval a.s. The process $\left\{X_{t}^{1}\right\}$ is a Lévy process with the triplet $(0,0, v)$. Let

$$
X_{t}^{2}(\omega)=X_{t}(\omega)-X_{t}^{1}(\omega)
$$

Then $\left\{X_{t}^{2}\right\}$ is a Lévy process continuous in $t$ a.s. with the characteristic triplet $(A, \gamma, 0)$. The two processes $\left\{X_{t}^{1}\right\}$ and $\left\{X_{t}^{2}\right\}$ are independent.

In general, we may call $\left\{X_{t}^{1}\right\}$ and $\left\{X_{t}^{2}\right\}$ the jump part and the continuous part of $\left\{X_{t}\right\}$, respectively, but the sum of the jumps actually diverges a.s. if $\int_{|x| \leq 1}|x| v(d x)=\infty$ and we need the centering term $-x v(d(x))$, the so-called compensator, in order to achieve the convergence. Notice also that an important result from the Lévy-Itô decomposition is the relationship between Lévy processes and semimartingales. Using directly the Lévy-Itô decomposition we can conclude that every Lévy process is a semimartingale (cf. Jacod and Shiryaev (1987), Corollary II.4.19.).

\footnotetext{
${ }^{2}$ Recall that $\mathrm{t}$ is called fixed time of discontinuity of a process $X$ if $P\left[\triangle X_{t} \neq 0\right]>0$
} 


\subsection{Definition and Properties of LIBOR Additive Processes}

\subsubsection{The LIBOR additive process}

In this section we define a stochastic process, the LIBOR additive process, that will drive the riskneutral dynamics of instantaneous forward rates with independent but piecewise stationary increments (this means that it is stationary inside of each time interval, usually 6 or 12 months, defined by the tenor of the LIBOR rate). We will see that this generalization allows taking into account deterministic time inhomogeneities: the parameters describing the local behavior will now be time-dependent but nonrandom. Therefore, all definitions below will be applied to the time interval $t \in I=\left[0, T^{\star}\right] \subset \mathbb{R}_{+}$, where $T^{\star}$ is a fixed time horizon with $T^{\star}>0$. Also, let $\mathcal{T}$ be the family of all finite subsets of $I \subset \mathbb{R}_{+}$. For a predetermined collection of dates $J=\left\{T_{0}, T_{1}, \ldots, T_{n}\right\} \in \mathcal{T}$, such that $0=T_{0}<T_{1}<\ldots<T_{n}=T^{\star}$ with $J \subset I$, let $\delta_{j}=T_{j+1}-T_{j}$ denote the length or "tenor" of the $j$-th interval.

Definition 10 A stochastic process $G=\left\{G_{t}: 0 \leq t \leq T^{\star}\right\}$ on $\mathbb{R}^{d}$ is a LIBOR additive process in law if the following conditions are satisfied:

$(L A P 1) G_{0}=0$ a.s.

(LAP2) $G$ is a process with the independent increment property, i.e., for any choice of $m \in \mathbb{N}^{+}$and $0 \leq t_{1} \leq \ldots \leq t_{m} \leq T^{\star}$, the variables $G\left(t_{1}\right), G\left(t_{2}\right)-G\left(t_{1}\right), \ldots, G\left(t_{m}\right)-G\left(t_{m-1}\right)$ are independent.

(LAP3) $G$ is stochastically continuous or continuous in probability (but it may have discontinuous trajectories).

(LAP4) There exist $0=T_{0}<T_{1} \ldots<T_{n}=T^{\star}$, such that $G$ is a process with piecewise stationary increments, or temporally homogeneous in $\left[T_{j}, T_{j+1}\right)$, for any $j=0,1, \ldots, n-1$.

Definition 11 A stochastic process $\left\{G_{t}: 0 \leq t \leq T^{\star}\right\}$ on $\mathbb{R}^{d}$ is a LIBOR additive process if it satisfies $(L A P 1)$ to $(L A P 4)$ and if, additionally, there is $\Omega_{0} \in \mathcal{F}$ with $P\left[\Omega_{0}\right]=1$ such that, for every $\omega \in$ $\Omega_{0}, G_{t}(\omega)$ is right-continuous in $t \geq 0$ and has left-limits in $t>0$ ("càdlàg" process).

Notice that, according to Section 1.3, a Lévy process is defined as a stochastic process with stationary independent increments which is continuous in probability (but may have discontinuous trajectories). While Lévy processes offer nice features in terms of analytical tractability, the constraints of stationarity of their increments prove to be rather restrictive.

- The first advantage of our approach is that it allows for preserving the tractability of Lévy processes while enabling us to model the whole range of cap/floors or swaptions volatilities across strikes and maturities.

- A second advantage is that the property of piecewise stationarity is the usual performance that we suppose in the discretised version of the LIBOR market model where the tenor structure plays a relevant role, and it will be a key issue in our calibration procedure and credit risk modelling.

\subsubsection{Properties of LIBOR additive processes}

In this section, our aim is to briefly describe some relevant probabilistic properties ${ }^{3}$ of this stochastic process, and specifically, some properties related with the infinite divisibility of its distribution, self-

\footnotetext{
${ }^{3}$ Theoretical and empirical justification of the financial relevance for these properties can be found in Carr, Geman, Madan and Yor (2002).
} 
similarity, stability and self-decomposability.

\section{Infinite divisibility of LIBOR additive processes}

Recall that, for any $n \in \mathbb{N}_{+}$, we denoted by $\mu^{n *}$ or $\mu^{n}$ the $n$-fold convolution of a probability measure $\mu$ with itself, that is

$$
\mu^{n}=\mu^{n *}=\underbrace{\mu * \ldots * \mu}_{n \text { times }}
$$

$\mu$ on $\mathbb{R}^{d}$ is an infinitely divisible probability measure if, for any positive integer $n$, there is a probability measure $\mu_{(n)}$ on $\mathbb{R}^{d}$ such that $\mu=\mu_{(n)}^{n *}$, or in other words, $\mu$ can be expressed as the $n$-th convolution power of $\mu_{(n)}$. Equivalently, in terms of random variables, we say that $X$ is infinitely divisible if for all $n \in \mathbb{N}_{+}$, there exist i.i.d. random variables $X_{1}^{(n)}, \ldots, X_{n}^{(n)}$ such that

$$
X \stackrel{d}{=} X_{1}^{(n)}+\ldots+X_{n}^{(n)}
$$

Proposition 12 The following statements are equivalent:

1. $X$ is infinitely divisible

2. $\mu_{X}$ has a convolution $n$-th root that is itself the law of a random variable for each $n \in \mathbb{N}$

3. $\hat{\mu}_{X}(z)$ has an $n$-th root that is itself a characteristic function of a random variable for each $n \in \mathbb{N}$.

Proof. $(1) \Longrightarrow(2)$ The common law of the $X_{j}^{(n)}$ is the required convolution $n$-th root

$(2) \Longrightarrow(3)$ Let $X^{(n)}$ be a random variable with law $\left(\mu_{X}\right)^{1 / n}$ then we have for each $z \in \mathbb{R}^{d}$ :

$$
\begin{aligned}
\hat{\mu}_{X}(z) & =\int \ldots \int e^{i\left(z, x_{1}+\ldots+x_{n}\right)}\left(\mu_{X}\right)^{1 / n}\left(d x_{1}\right) \ldots\left(\mu_{X}\right)^{1 / n}\left(d x_{n}\right) \\
& =\left(\hat{\mu}_{X}(z)^{(1 / n)}\right)^{n}
\end{aligned}
$$

where $\hat{\mu}_{X}(z)^{(1 / n)}=\int_{\mathbb{R}^{d}} e^{i\left(z, x_{j}\right)}\left(\mu_{X}\right)^{1 / n}\left(d x_{j}\right)$ and the required result follows.

$(3) \Longrightarrow(1)$ Choose $X_{1}^{(n)}, \ldots, X_{n}^{(n)}$ to be independent copies of the given random variable, then we have

$$
\mathbb{E}\left(e^{i(z, X)}\right)=\mathbb{E}\left(e^{i\left(z, X_{1}^{(n)}\right)}\right) \cdot \ldots \cdot \mathbb{E}\left(e^{i\left(z, X_{n}^{(n)}\right)}\right)=\mathbb{E}\left(e^{i\left(z, X_{1}^{(n)}+\ldots+X_{n}^{(n)}\right)}\right)
$$

Now, let us extend the concept of infinite divisibility to stochastic processes.

Definition 13 A stochastic process $X=\left\{X_{t}: t \geq 0\right\}$ on $\mathbb{R}^{d}$ is infinitely divisible if all finitedimensional marginals of $X$ are infinitely divisible, that is, for any choice of distinct $t_{1}, \ldots, t_{m} \in\left[0, T^{\star}\right]$ with $m \in \mathbb{N}_{+},\left(X_{t_{j}}\right)_{1 \leq j \leq m}$ is infinitely divisible. Here $\left(X_{t_{j}}\right)_{1 \leq j \leq m}$ is an $\mathbb{R}^{m d}$-valued random variable.

Notice that it is not difficult to check that a Lévy process is an infinitely divisible process (see Proposition 3 in Section 1.1.3) due to the homogeneity property. 
The LIBOR additive process does not preserve the homogeneity property anymore (see (LAP4) in Definition 10) as in the Lévy case. Actually, it is only a piecewise stationary process. However, we can attempt to prove the infinite divisibility property using independent increments (see (LAP2) in Definition 10) and stochastic-continuity property (LAP3), following a similar reasoning as in Sato (1999). It is based on one of the fundamental limit theorems on sums of independent random variables, conjectured by Kolmogorov and proved by Khintchine.

Definition 14 A double sequence of random variables $\left\{Z_{i}^{(n)}: i=1,2, \ldots, r_{n} ; n=1,2, \ldots\right\}$ in $\mathbb{R}^{d}$ is called a null array if for each fixed $n, Z_{1}^{(n)}, Z_{2}^{(n)}, \ldots, Z_{r_{n}}^{(n)}$ are independent and if, for any $\varepsilon>0$,

$$
\lim _{n \rightarrow \infty} \max _{1 \leq i \leq r_{n}} \mathbb{P}\left[\left|Z_{i}^{(n)}\right|>\varepsilon\right]=0
$$

The sums $S_{n}=\sum_{i=1}^{r_{n}} Z_{i}^{(n)}, n=1,2, \ldots$, are called the row sums.

Theorem 15 (Khintchine (1937) ) Let $\left\{Z_{i}^{(n)}\right\}$ be a null array in $\mathbb{R}^{d}$ with row sums $S_{n}$. If, for some $b_{n} \in \mathbb{R}^{d}, n=1,2, \ldots$, the distributions of $S_{n}-b_{n}$ converge to a distribution $\mu$, then $\mu$ is infinitely divisible (id).

Proof. cf. Khintchine (1937)

Lemma 16 The LIBOR additive process in law $G=\left\{G_{t}: t \geq 0\right\}$ is uniformly stochastically continuous on any finite interval $\left[0, T^{\star}\right]$, that is, for every $\varepsilon>0$ and $\eta>0$, there is $\delta>0$ such that, if $s$ and $t$ are in $\left[0, T^{\star}\right]$ and satisfy $|s-t|<\delta$, then $\mathbb{P}\left[\left|G_{s}-G_{t}\right|>\varepsilon\right]<\eta$.

Proof. Fix $\varepsilon>0$ and $\eta>0$. From property $(L A P 3)$ in Definition 10, we have that for any $t$ there is $\delta_{t}>0$ such that

$$
\mathbb{P}\left[\left|G_{s}-G_{t}\right|>\varepsilon / 2\right]<\eta / 2 \text { for }|s-t|<\delta_{t}
$$

Let $I_{t}=\left(t-\delta_{t} / 2, t+\delta_{t} / 2\right)$, then $\left\{I_{t}: t \in\left[0, T^{\star}\right]\right\}$ covers the interval $\left[0, T^{\star}\right]$

Hence there is a finite subcovering $\left\{I_{t_{j}}: j=1, \ldots, n\right\}$ of $\left[0, T^{\star}\right]$.

Let $\delta$ be the minimum of $\delta_{t_{j}} / 2, j=1, \ldots, n$. If $|s-t|<\delta$ and $s, t \in\left[0, T^{\star}\right]$ then $t \in I_{t_{j}}$ for some $j$. Hence $\left|s-t_{j}\right|<\delta_{t_{j}}$, and

$$
\mathbb{P}\left[\left|G_{s}-G_{t}\right|>\varepsilon\right] \leq \mathbb{P}\left[\left|G_{s}-G_{t_{j}}\right|>\varepsilon / 2\right]+\mathbb{P}\left[\left|G_{t}-G_{t_{j}}\right|>\varepsilon / 2\right]<\eta
$$

And finally, we can state the result that we look for.

Theorem 17 If $G=\left\{G_{t}: t>0\right\}$ is a LIBOR additive process in law on $\mathbb{R}^{d}$, then for every $t$ the distribution of $G_{t}$ is infinitely divisible.

Proof. Fix a time interval $[0, t]$ with $t>0$. Let $t_{i}^{(n)}=i t / n$ for $i=0,1,2, \ldots, n$ and $n=1,2, \ldots$ Set

$$
Z_{i}^{(n)}=G\left(t_{i}^{(n)}\right)-G\left(t_{i-1}^{(n)}\right) \quad \text { for } i=1,2, \ldots, n
$$


Let us recall that Khintchine's theorem shows that $G(t)$ is infinitely divisible if $\left\{\left\{Z_{i}^{(n)}\right\}_{i=1}^{n}\right\}_{n=1}^{\infty}$ is null-array. To prove that $Z_{i}^{(n)}$ is a null array (with $r_{n}=n$ ) we use the uniform stochastic continuity from Lemma $16:$ when $n \rightarrow \infty$

$$
\max _{1 \leq i \leq n} \mathbb{P}\left\{\left|Z_{i}^{(n)}\right|>\varepsilon\right\} \leq \sup _{1 \leq i \leq n} \mathbb{P}\left[\left|G\left(t_{i}^{(n)}\right)-G\left(t_{i-1}^{(n)}\right)\right|>\varepsilon\right] \underset{n \rightarrow \infty}{\rightarrow} 0
$$

Therefore $\left\{\left\{Z_{i}^{(n)}\right\}_{i=1}^{n}\right\}_{n=1}^{\infty}$ is a null-array. Hence we can apply Theorem 15 or Khintchine's Theorem with $\mu=\mathbb{P}_{G_{t}}$ and $b_{n}=0$.

Remark 18 Notice the strong relationship that exists between the concepts of independent increments and stochastic continuity with infinitely divisible distributions. These concepts will be helpful to work not only with the concept of efficient financial markets, but also with limit law distributions class (see Theorem 25).

\section{Stability and self-decomposability of LIBOR additive process}

If $\left\{W_{t}: t \geq 0\right\}$ is the Brownian motion on $\mathbb{R}^{d}$ then for any $a>0$ the process $\left\{W_{a t}: t \geq 0\right\}$ is identical in law with the process $\left\{a^{1 / 2} W_{t}: t \geq 0\right\}$. This means that any change of the time scale for the Brownian motion has the same effect as some change of the spatial scale. This property is usually called selfsimilarity. There are many self-similar Lévy processes other than the Brownian motion. They constitute an important class called strictly stable processes.

Roughly speaking, stable processes are Lévy processes for which a change of time scale has the same effect as a change of spatial plus a linear drift. In other words, they are invariant in distribution under an appropriate scaling of time and space. They are important in probability because of their connection to limit theorems (see Lamperti (1962)) and they are of great interest in financial modelling.

In this subsection, we define both concepts and some extensions, and we determine the conditions for the self-similarity of LIBOR additive processes.

Definition 19 Let $\mu$ be an infinitely divisible probability measure on $\mathbb{R}^{d}$. It is called stable if, for any $a>0$, there are $b>0$ and $c \in \mathbb{R}^{d}$ such that

$$
\hat{\mu}(z)^{a}=\hat{\mu}(b z) e^{i\langle c, z\rangle}
$$

It is strictly stable if, for any $a>0$, there is $b>0$ such that

$$
\hat{\mu}(z)^{a}=\hat{\mu}(b z)
$$

Definition 20 Let $\left\{G_{t}: t \geq 0\right\}$ be a LIBOR additive process on $\mathbb{R}^{d}$. It is called a stable or strictly stable process if the distribution of $G_{t}$ at $t=1$ is stable or strictly stable, respectively.

Definition 21 Let $\left\{X_{t}: t \geq 0\right\}$ be a stochastic process on $\mathbb{R}^{d}$. It is called self-similar if, for any $a>0$, there is $b>0$ such that

$$
\left\{X_{a t}: t \geq 0\right\} \stackrel{d}{=}\left\{b X_{t}: t \geq 0\right\}
$$

It is called broad-sense self-similar if, for any $a>0$ there is $b>0$ and a function $c(t)$ from $[0, \infty)$ to $\mathbb{R}^{d}$ such that

$$
\left\{X_{a t}: t \geq 0\right\} \stackrel{d}{=}\left\{b X_{t}+c(t): t \geq 0\right\}
$$


Now let us consider the Generalized Central Limit problem. Let $Z_{1}, Z_{2}, \ldots$ be an independent, identically distributed sequence of random variables. Let $S^{(m)}$ be defined as the sum of $m$ of these independent identically distributed random variables. We are interested in the case where there exists the following relationship

$$
\lim _{m \rightarrow \infty} P\left(b^{(m)} S^{(m)}+c^{(m)} \leq x\right)=P(X \leq x) \text { for all } x \in \mathbb{R}^{d}
$$

Theorem 22 A probability measure $\mu$ on $\mathbb{R}^{d}$ is stable if and only if there is a random walk $\left\{S^{(m)}\right\}, b^{(m)}>$ 0 and $c^{(m)} \in \mathbb{R}^{d}$ such that $\mathbb{P}_{b^{(m)} S^{(m)}+c^{(m)}} \rightarrow \mu$ as $m \rightarrow \infty$. And in particular, it is strictly stable if each $c^{(m)}=0$.

Proof. cf. Sato (1999) Theorem 15.7.

It is immediate to see that it is only possible to talk about stability of the LIBOR additive process inside of the interval $\left[T_{j}, T_{j+1}\right)$ with $j=0, \ldots, m$, where the increments are identically distributed. But we can generalize the definition of a stable process if we weaken the conditions on the process in the central limit theorem by requiring these to be independent but no longer necessarily identically distributed. This is the case of the LIBOR additive process, in which case the limiting process is called self-decomposable.

Definition 23 Let $\mu$ be a probability measure on $\mathbb{R}^{d}$. It is called self-decomposable if for any $c \in(0,1)$ there is a probability measure $\rho^{(c)}$ on $\mathbb{R}^{d}$ such that

$$
\hat{\mu}(z)=\hat{\mu}(c z) \hat{\rho}^{(c)}(z)
$$

It is called semi-selfdecomposable if there are some $c \in(0,1)$ and some infinitely divisible probability measure $\rho^{(c)}$ satisfying (1.10).

Definition 24 A stochastic process $X=\left\{X_{t}: t \geq 0\right\}$ on $\mathbb{R}^{d}$ is self-decomposable if all finite-dimensional marginals of $X$ are self-decomposable that is, for any choice of distinct $t_{1}, \ldots, t_{m} \in\left[0, T^{\star}\right],\left(X_{t_{j}}\right)_{1 \leq j \leq m}$ is self-decomposable. Here $\left(X_{t_{j}}\right)_{1 \leq j \leq m}$ is an $\mathbb{R}^{m d}$-valued random variable.

The class of self-decomposable distributions is obtained as a class of limit distributions described below.

Theorem $25(i)$ Let $\left\{X_{i}^{(m)}: i=1,2, \ldots, m\right\}$ be independent random variables on $\mathbb{R}^{d}$ and $S^{(m)}=\sum_{i=1}^{m} X_{i}^{(m)}$. Let $\mu$ be a probability measure on $\mathbb{R}^{d}$. Suppose that there are $b^{(m)}>0$ and $c^{(m)} \in \mathbb{R}^{d}$ for $m=1,2, \ldots$ such that

$$
\mathbb{P}_{b^{(m)} S^{(m)}+c^{(m)}} \underset{m \uparrow \infty}{\longrightarrow} \mu
$$

and that $t^{4}$

$$
\left\{b^{(m)} X_{i}^{(m)}: i=1, \ldots, m ; m=1,2, \ldots\right\} \text { is a null array }
$$

or equivalently

$$
\lim _{m \uparrow \infty} \max _{1 \leq i \leq m} \mathbb{P}\left\{\left|b^{(m)} X_{i}^{(m)}\right|>\varepsilon\right\}=0
$$

Then $\mu$ is self-decomposable.

(ii) For any self-decomposable distribution $\mu$ on $\mathbb{R}^{d}$ we can find $\left\{X_{i}^{(m)}\right\}$ independent, $b^{(m)}>0$ and $c^{(m)} \in \mathbb{R}^{d}$ satisfying (1.11) and (1.12).

\footnotetext{
${ }^{4}$ See Definition 14.
} 
Proof. cf. Sato (1999) Theorem 15.3

Remark 26 Limit laws are probably the best explanation for the wide-spread use of the Gaussian law in the study of financial markets. The self-decomposable laws are limit laws and this is also their appeal. Notice the relationship that appears between the concepts of independent increments and stochastic continuity with self-decomposable distributions.

Theorem 27 If $G=\left\{G_{t}: t>0\right\}$ is a LIBOR additive process in law on $\mathbb{R}^{d}$ then for every $t$, the distribution of $G_{t}$ is self-decomposable.

Proof. Fix a time interval $[0, t]$ with $t>0$. Let $t_{i}^{(n)}=i t / n$ for $i=1,2, \ldots, n$ and $n=1,2, \ldots$ Let us define $Z_{i}^{(n)}$ as

$$
Z_{i}^{(n)}=G\left(t_{i}^{(n)}\right)-G\left(t_{i-1}^{(n)}\right) \quad \text { for } i=1,2, \ldots, n
$$

Let us recall that $\left\{\left\{Z_{i}^{(n)}\right\}_{i=1}^{n}\right\}_{n=1}^{\infty}$ is a null-array, by Definition 14 and uniform stochastic continuity property given in Lemma 16. The assertion follows as in the proof of Theorem 17 . Therefore, by direct application of Theorem 25, we conclude that the LIBOR additive process in law has a selfdecomposable distribution.

Theorem 28 A stochastic process $X=\left\{X_{t}: t \geq 0\right\}$ on $\mathbb{R}^{d}$ is self-decomposable if and only if for every $c \in(0,1)$,

$$
X \stackrel{d}{=} c X^{\prime}+U^{(c)}
$$

where $X^{\prime}=\left\{X_{t}^{\prime}: t \geq 0\right\}$ is a version of $X$, and $U^{(c)}=\left\{U_{t}^{(c)}: t \geq 0\right\}$ is a stochastic process on $\mathbb{R}^{d}$ and $X^{\prime}$ and $U^{(c)}$ are independent. The law of $U^{(c)}$ is uniquely determined by $c$ and the law of $X$, and $U^{(c)}$ is an infinitely divisible process.

\section{Proof. cf. Barndorff-Nielsen, Maejima and Sato (2006).}

Theorem 29 Let $G$ be a self-decomposable LIBOR additive process on $\mathbb{R}^{d}$, then for every $c \in(0,1)$ the process $U^{(c)}$ can be chosen to be a LIBOR additive process.

Proof. Notice that the LIBOR additive process $G$ is a self-decomposable, according with Theorem 27. Let us denote $\mu_{t}=\mathcal{L}\left(G_{t}\right)$ and $\mu_{s, t}=\mathcal{L}\left(G_{t}-G_{s}\right)$ for $0 \leq s \leq t$. According to Theorem 28, fix $c \in(0,1)$ and denote $U_{t}=U_{t}^{(c)}, \rho_{t}=\mathcal{L}\left(U_{t}\right)$, and $\rho_{s, t}=\mathcal{L}\left(U_{t}-U_{s}\right)$ for $0 \leq s \leq t$.

Then

$$
\hat{\mu}_{t}(z)=\hat{\mu}_{s}(z) \hat{\mu}_{s, t}(z)
$$

where

$$
\begin{aligned}
\hat{\mu}_{t}(z) & =\hat{\mu}_{t}(c z) \hat{\rho}_{t}(z) \\
\hat{\mu}_{s, t}(z) & =\hat{\mu}_{s, t}(c z) \hat{\rho}_{s, t}(z)
\end{aligned}
$$

Notice that $\hat{\rho}_{s, t} \rightarrow 1$ when $s \downarrow t$ or $t \uparrow s$. It follows that $U=U^{(c)}$ is stochastically continuous (property $(L A P 3)$ in Definition 10$)$. Obviously $U_{0}=0$ a.s (LAP1).

In order to prove that $U$ is a LIBOR additive process in law, according to Definition 10, additionally we need the independent increments $(L A P 2)$ and piecewise stationary property $(L A P 4)$. 
Let $0=t_{0}<t_{1}<\ldots<t_{n}=T^{\star}$ and $z_{1}, \ldots, z_{n} \in \mathbb{R}^{d}$ and $z_{n+1}=0$, then

$$
\begin{aligned}
\mathbb{E}\left[\exp \left(i \sum_{j=1}^{n}\left\langle z_{j}, U_{t_{j}}-U_{t_{j-1}}\right\rangle\right)\right] & =\mathbb{E}\left[\exp \left(i \sum_{j=1}^{n}\left\langle z_{j}-z_{j+1}, U_{t_{j}}\right\rangle\right)\right] \\
& =\mathbb{E}\left[\exp \left(i \sum_{j=1}^{n}\left\langle z_{j}-z_{j+1}, G_{t_{j}}\right\rangle\right)\right] / \mathbb{E}\left[\exp \left(i \sum_{j=1}^{n}\left\langle z_{j}-z_{j+1}, c G_{t_{j}}^{\prime}\right\rangle\right)\right] \\
& =\mathbb{E}\left[\exp \left(i \sum_{j=1}^{n}\left\langle z_{j}, G_{t_{j}}-G_{t_{j-1}}\right\rangle\right)\right] / \mathbb{E}\left[\exp \left(i \sum_{j=1}^{n}\left\langle z_{j}, c G_{t_{j}}^{\prime}-c G_{t_{j-1}}^{\prime}\right\rangle\right)\right] \\
& =\prod_{j=1}^{n} \mathbb{E}\left[\exp \left(i\left\langle z_{j}, G_{t_{j}}-G_{t_{j-1}}\right\rangle\right)\right] / \prod_{j=1}^{n} \mathbb{E}\left[\exp \left(i\left\langle z_{j}, c G_{t_{j}}^{\prime}-c G_{t_{j-1}}^{\prime}\right\rangle\right)\right] \\
& =\prod_{j=1}^{n} \mathbb{E}\left[\exp \left(i\left\langle z_{j}, U_{t_{j}}-U_{t_{j-1}}\right\rangle\right)\right]
\end{aligned}
$$

This shows $(L A P 2)$ and $(L A P 4)$, therefore $U$ is a LIBOR additive process in law. 


\subsection{Existence of LIBOR additive processes}

The aim of this section is to provide a simple proof of the existence of the LIBOR additive process according to the definition given in section 1.2.1 (Definition 10). A direct construction in the Skorohod space is described in the current section, and it is based directly on the fact that the LIBOR additive process, in terms of trajectories, can be observed as a piecewise stationary Lévy process. Therefore the existence of the Lévy process guarantees the existence of LIBOR additive process in the Skorohod space.

Recall that we are given a time interval $\left[0, T^{\star}\right]$ with $T^{\star}$ fixed. Also, notice that, given $n \in \mathbb{N}_{+}$, we have a predetermined collection of fixed dates $0=T_{0}<T_{1}<\ldots<T_{n}=T^{\star}$. Let us define $\eta$ as a right-continuous function $\eta:\left[0, T_{n}\right] \rightarrow\{0,1, \ldots, n\}$ by taking $\eta(t)$ to be $\eta(t)=\sup \left\{0 \leq i \leq n: T_{i} \leq t\right\}$.

Let $G^{(j)}$, with $0 \leq j \leq n$, be a family of $n+1$ independent Lévy processes such that $G^{(j)}$ has the triplet $\left(\gamma_{j}, A_{j}, v_{j}\right)$. Set for $0 \leq t \leq T^{\star}$

$$
G(t)=\sum_{j=0}^{\eta(t)}\left(G^{(j)}\left(T_{j+1} \wedge t\right)-G^{(j)}\left(T_{j}\right)\right)
$$

Then $G(t)$ has characteristic function, due to the independence of the $G^{(j)}$ 's:

$$
e^{T_{1} \psi_{1}(z)+\left(T_{2}-T_{1}\right) \psi_{2}(z)+\ldots+\left(t-T_{\eta(t)}\right) \psi_{\eta(t)}(z)}=e^{\sum_{j \leq \eta(t)}\left(\left(T_{j+1} \wedge t\right)-T_{j}\right) \psi_{j}(z)}
$$

where $\psi_{j}(z)$ is the Lévy exponent of $G^{(j)}$ (see Theorem 31 for further details). Since $G^{(j)}$ has sample paths in the Skorohod space, it immediately follows from the above construction that the same is true for $G$. Let us prove that $G$ is a LIBOR additive process.

Theorem 30 Let $\left\{G_{t}, t \geq 0\right\}$ be a stochastic process on $\mathbb{R}^{d}$, defined by $(1.15)$. Then $G_{t}$ is a LIBOR additive process.

Proof. Notice that to prove that $G$ is a LIBOR additive process, we just need to prove properties $(L A P 1)$ to $(L A P 4)$ in Definition 10 as properties of $G$. (LAP1) is obvious taking into account property $(L 1)$ in Definition 2 of Lévy process in law. To prove $(L A P 2)$ in Definition 10, notice that using the property $(L 2)$ in Definition 2, we have that for any $s, t \in\left[0, T^{\star}\right]$ belonging to the same sub-interval $T_{j} \leq s \leq t<T_{j+1}$ :

$$
G_{t}-G_{s}=G_{t}^{(j)}-G_{s}^{(j)}
$$

is identical with the increment of $G^{(j)}$ that has independent increments by $(L 2)$ in Definition 2 of Lévy process in law. If $s<T_{j} \leq t<T_{j+1}$ are in adjacent intervals, then

$$
\begin{aligned}
G_{t}-G_{s} & =G_{t}-G_{T_{j}}^{(j)}+G_{T_{j}}^{(j)}-G_{s} \\
& =G_{t}^{(j+1)}-G_{T_{j}}^{(j+1)}+\left(G_{T_{j}}^{(j)}-G_{T_{j-1}}^{(j)}\right)-\left(G_{s}^{(j)}-G_{T_{j-1}}^{(j)}\right) \\
& =\left(G_{t}^{(j+1)}-G_{T_{j}}^{(j+1)}\right)+\left(G_{T_{j}}^{(j)}-G_{s}^{(j)}\right)
\end{aligned}
$$

Hence this increment may be decomposed into two independent increments over adjacent intervals. These two facts finally lead to the independence property of the adjacent increments and hence to increments in general.

Since each $G$ has no fixed discontinuities (Theorem 8), the stochastic continuity (LAP3) is guaranteed, and finally $(L A P 4)$ is obvious using (1.15). 


\subsection{Characterization of LIBOR additive processes}

The following results recall the representation of characteristic functions of infinitely divisible distributions, briefly shown in Section 1.1.3. The Lévy-Khintchine formula was obtained on $\mathbb{R}$ around 1930 by De Finetti and Kolmogorov in special cases, and then mentioned by Lévy (1934) in the general case. It was immediately extended to $\mathbb{R}^{d}$. This theorem is essential to the whole theory, and a simpler proof was given by Khintchine (1937) and Gnedenko and Kolmogorov (1954). Here we show a detailed version, however, the proof is omitted.

Theorem 31 (Lévy-Khintchine) (i) If $\mu$ is an infinitely divisible distribution on $\mathbb{R}^{d}$, then its characteristic function $\hat{\mu}(z)$ has the form

$$
\hat{\mu}(z)=\exp [\psi(z)]
$$

where the Lévy exponent $\psi(z)$ with $z \in \mathbb{R}^{d}$ equals

$$
\psi(z)=i\langle\gamma, z\rangle-\frac{1}{2}\langle z, A z\rangle+\int_{\mathbb{R}^{d}}\left(e^{i\langle z, g\rangle}-1-i\langle z, g\rangle 1_{\{|g| \leq 1\}}\right) v(d g)
$$

and where $A$ is a symmetric nonnegative-definite $d \times d$ matrix, $\gamma \in \mathbb{R}^{d}, v$ is a Radon measure on $\mathbb{R}^{d} \backslash\{0\}$ and $g \in \mathbb{R}^{d}$ satisfying

$$
v(\{0\})=0 \text { and } \int_{\mathbb{R}^{d}}\left(|g|^{2} \wedge 1\right) v(d g)<\infty
$$

(ii) The representation of $\hat{\mu}(z)$ in $(i)$ by $A, v$, and $\gamma$ is unique.

(iii) Conversely, if $A$ is a symmetric nonnegative-definite $d \times d$ matrix, $v$ is a measure satisfying (1.22) and $\gamma \in \mathbb{R}^{d}$, then there exists an infinitely divisible distribution $\mu$ whose characteristic function is given by (1.20).

Proof. cf. Sato (1999) Theorem 8.1.

Definition 32 We call $(A, v, \gamma)$ in Theorem 31 the generating triplet of $\mu$. The $A$ and the $v$ are called, respectively, the Gaussian covariance matrix and the Lévy measure of $\mu$. When $A=0, \mu$ is called purely non-gaussian.

Corollary 33 If $\mu$ has the generating triplet $(A, v, \gamma)$, then $\mu^{t}$ has the generating triplet $(t A, t v, t \gamma)$.

Now, let $G=\left\{G_{t} ; t \geq 0\right\}$ be the LIBOR additive process with a given tenor structure $0=T_{0}<T_{1}<$ $\ldots<T_{n}=T^{\star}$ with $T^{\star}$ fixed. Let us recall that, given a set of $n$-Lévy processes, the LIBOR additive process can be constructed as $G_{t}=\sum_{j=0}^{\eta(t)}\left(G^{(j)}\left(T_{j+1} \wedge t\right)-G^{(j)}\left(T_{j}\right)\right)$ (in 1.15). Let us call $\mu^{(j)}$ as the distribution (or law) associated to the Lévy process $G^{(j)}$, and additionally, let us define $\mu_{T_{j}, T_{j+1}}^{(j)}$ and $\hat{\mu}_{T_{j}, T_{j+1}}^{(j)}$ as the distribution and characteristic function respectively associated to the increment $\left(G^{(j)}\left(T_{j+1}\right)-G^{(j)}\left(T_{j}\right)\right)$. The next theorem shows how the distribution function of $G_{t}$, is characterized by the sequence of triplets $\left\{\left(A_{j}, v_{j}, \gamma_{j}\right)\right\}_{j \leq \eta(t)}$.

Theorem 34 Ad-dimensional process $G=\left\{G_{t} ; t \geq 0\right\}$ is a LIBOR additive process if and only if it is a semimartingale admitting a sequence of triplets $\left\{\left(A_{j}, v_{j}, \gamma_{j}\right)\right\}_{j \leq \eta(t)}$ such that for all $t \in \mathbb{R}^{+}$and 
$z \in \mathbb{R}^{d}$ we have

$$
\hat{\mu}_{t}(z)=\prod_{j \leq \eta(t)} \hat{\mu}_{T_{j}, t \wedge T_{j+1}}^{(j)}(z)
$$

where $\left(A_{j}, \nu_{j}, \gamma_{j}\right)$ is the triplet associated with the characteristic function of $\mu_{T_{j}, T_{j+1}}$, for any $j=$ $0,1, \ldots, \eta(t)$.

Proof. For any $t \in\left[0, T^{\star}\right]$ and given a tenor structure $0=T_{0}<T_{1} \ldots<T_{n}=T^{\star}$, then with $G_{t}=\sum_{j \leq \eta(t)}\left(G^{(j)}\left(T_{j+1} \wedge t\right)-G^{(j)}\left(T_{j}\right)\right)$ and using the independent increments property we have

$$
\begin{aligned}
& \mathbb{E}\left[f\left(G_{T_{0}}, \ldots, G_{T_{\eta(t)}}, G_{t}\right)\right] \\
= & \int \ldots \int f\left(g_{0}, g_{0}+g_{1}, \ldots, g_{0}+\ldots+g_{\eta(t)}\right) \mu^{(0)}\left(d g_{0}\right) \times \mu^{(1)}\left(d g_{1}\right) \times \ldots \times \mu^{(\eta(t))}\left(d g_{\eta(t)}\right)
\end{aligned}
$$

for any bounded measurable function $f$. Let $z_{1}, \ldots, z_{n} \in \mathbb{R}^{d}$ and

$$
f\left(g_{0}, g_{1}, \ldots, g_{\eta(t)}\right)=\exp \left(i \sum_{j=0}^{\eta(t)}\left\langle z_{j}, g_{j+1}-g_{j}\right\rangle\right)
$$

Therefore

$$
\begin{aligned}
\hat{\mu}_{t}(z) & =\mathbb{E}\left[\exp \left(i \sum_{j=0}^{\eta(t)}\left\langle z_{j}, g_{j+1}-g_{j}\right\rangle\right)\right] \\
& =\int \ldots \int\left[\exp \left(i \sum_{j=0}^{\eta(t)}\left\langle z_{j}, g_{j+1}-g_{j}\right\rangle\right)\right] \mu_{0}^{(0)}\left(d g_{0}\right) \times \mu_{T_{0}, T_{1}}^{(1)}\left(d g_{1}\right) \times \ldots \times \mu_{T_{\eta(t)}, t}^{(\eta(t))}\left(d g_{\eta(t)}\right) \\
& =\prod_{j \leq \eta(t)} \int \ldots \int \exp \left(i\left\langle z_{j}, g_{j+1}-g_{j}\right\rangle\right) \mu_{T_{j}, T_{j+1} \wedge t}^{(j)}\left(d g_{j}\right) \\
& =\prod_{j \leq \eta(t)} \mathbb{E}\left(\exp \left[i z\left(G_{t \wedge T_{j+1}}^{(j)}-G_{T_{j}}^{(j)}\right)\right]\right)
\end{aligned}
$$

and using Lévy-Khintchine Theorem 31.i) we have

$$
\begin{aligned}
\hat{\mu}_{t}(z) & =\exp \left[\sum_{j \leq \eta(t)}\left(t \wedge T_{j+1}-T_{j}\right) \Psi_{j}(z)\right] \\
& =\prod_{j \leq \eta(t)} \hat{\mu}_{T_{j}, t \wedge T_{j+1}}^{(j)}(z)
\end{aligned}
$$

where

$$
\begin{aligned}
\hat{\mu}_{T_{j}, T_{j+1}}^{(j)}(z) & =\mathbb{E}\left[\exp \left(i z\left(G_{T_{j+1}}^{(j)}-G_{T_{j}}^{(j)}\right)\right)\right] \\
& =\exp \left[\left(T_{j+1}-T_{j}\right) \psi_{j}(z)\right]
\end{aligned}
$$

and

$$
\psi_{j}(z)=i\left\langle\gamma_{j}, z\right\rangle-\frac{1}{2}\left\langle z, A_{j} z\right\rangle+\int_{\mathbb{R}^{d}}\left(e^{i\langle z, g\rangle}-1-i\langle z, g\rangle 1_{\{|g| \leq 1\}}\right) v_{j}(d g)
$$

The sufficient condition came as a direct consequence of (iii) in the Lévy-Khintchine theorem. 
Remark 35 Let $G=\left\{G_{t}: t>0\right\}$ be a LIBOR additive process. Notice that, given a tenor structure $0=T_{0}<T_{1}<\ldots<T_{n}=T^{\star}$ with $T^{\star}$ fixed, and a set of $n$ infinitely divisible measures $\left\{\mu_{0}, \mu_{1}, \ldots, \mu_{n}\right\}$ associated to this tenor structure, then, for any $s, t \in\left[0, T^{\star}\right]$ with $s<t, \mu_{s, t}$, the distribution of $G_{t}-G_{s}$, is uniquely determined in law by its sequence of triplets $\left\{\left(A_{j}, v_{j}, \gamma_{j}\right)\right\}_{\eta(s) \leq j \leq \eta(t)}$ (as a direct consequence from Theorem 31, ii) and Theorem 34). 


\subsection{Infinitesimal generators of LIBOR additive processes}

Here we turn our attention to the infinitesimal generator of the LIBOR additive process which will play an essential role in Chapter 2. This section is an attempt to gather some basic and typical results in order to introduce some of the results that appear in Chapter 2. It is not intended to give a systematic presentation of the most important results or to explain how to prove them; for these purposes the reader can find a more comprehensive picture in Ethier and Kurtz (1986) or Sato (1999).

This section has been divided in two subsections:

- In the first, we review a number of basic definitions and theorems related with the infinitesimal generator of its transition semigroup. Basically, the aim of this preliminary section is to give a brief introduction to the theory of semigroups of linear operators.

- In the second subsection, we apply directly these definitions to the LIBOR additive process. Further developments can be found in Chapter 2.

\subsubsection{Strongly continuous contraction semigroup and infinitesimal generator}

Let $\mathbb{B}$ be a real (or complex) Banach space. That is, $\mathbb{B}$ is a vector space over the real (or complex) scalar field equipped with a mapping $\|f\|$ from $\mathbb{B}$ into $\mathbb{R}$, called the norm, satisfying

$$
\begin{array}{lll}
\text { (1) }\|a f\|=|a|\|f\| & \text { for } f \in \mathbb{B}, a \in \mathbb{R}(\text { or } a \in \mathbb{C}) \\
\text { (2) } \quad\|f+g\| \leq\|f\|+\|g\| & \text { for } f, g \in \mathbb{B} \\
\text { (3) }\|f\|=0 & \text { if and only if } f=0
\end{array}
$$

such that if a sequence $\left\{f_{n}\right\}$ in $\mathbb{B}$ satisfies $\lim _{n, m \rightarrow \infty}\left\|f_{n}-f_{m}\right\|=0$, then there is $f \in \mathbb{B}$ with $\lim _{n \rightarrow \infty}\left\|f_{n}-f\right\|=$ 0 .

A linear operator $P$ in $\mathbb{B}$ is a mapping from a linear subspace $\mathcal{D}(P)$ of $\mathbb{B}$ into $\mathbb{B}$ such that

$$
P(a f+b g)=a P f+b P g \quad \text { for } f, g \in \mathcal{D}(P) \quad a, b \in \mathbb{R}(\text { or } a, b \in \mathbb{C})
$$

The set $\mathcal{D}(P)$ is called the domain of $P$.

A linear operator $P$ is called bounded if $\mathcal{D}(P)=\mathbb{B}$ and $\sup _{\|f\| \leq 1}\|P f\|$ called the norm of $P$ and denoted by $\|P\|$, is finite. A linear operator $P$ with $\mathcal{D}(P)=\mathbb{B}$ is bounded if and only if $P$ is continuous in the sense that $f_{n} \rightarrow f$ implies $P f_{n} \rightarrow P f$.

A linear operator $P$ is said to be closed if $f_{n} \in \mathcal{D}(P), f_{n} \rightarrow f$ and $P f_{n} \rightarrow g$ imply $f \in \mathcal{D}(P)$ and $P f=g$, in other words, if the graph of $P,\{(f, P f): f \in \mathcal{D}(P)\}$ is a closed set in $\mathbb{B} \times \mathbb{B}$. The set $\{P f: f \in \mathcal{D}(P)\}$ called the range of $P$, is denoted by $\mathcal{R}(P)$. The identity operator on $\mathbb{B}$ is denoted by $I$. A subset $\mathcal{D}_{1}$ of $\mathbb{B}$ is said to be dense in $\mathbb{B}$ if, for any $f \in \mathbb{B}$, there is a sequence $\left\{f_{n}\right\}$ in $\mathcal{D}_{1}$ such that $f_{n} \rightarrow f$.

Definition 36 A family $\left\{P_{t}: t \geq 0\right\}$ of bounded linear operator on $\mathbb{B}$ is called a strongly continuous semigroup if

$$
\begin{aligned}
& \text { (1) } P_{t} P_{s}=P_{t+s} \quad \text { for } t, s \in[0, \infty) \\
& \text { (2) } P_{0}=I \\
& \text { (3) } \lim _{t \downarrow 0} P_{t} f=f \quad \text { for any } f \in \mathbb{B}
\end{aligned}
$$


It is called a strongly continuous contraction semigroup if, moreover,

$$
\left\|P_{t}\right\| \leq 1
$$

Definition 37 The infinitesimal generator L of a strongly continuous contraction semigroup $\left\{P_{t}\right\}$ is defined by

$$
L f=\lim _{t \downarrow 0} \frac{1}{t}\left(P_{t} f-f\right)
$$

with $\mathcal{D}(L)$ being the set of $f$ such that the right-hand side of (1.26) exists.

One of the major theorem of the theory of semigroups of operators is as follows. It was independently proved by Hille (1948) and Yosida (1948), and the proof can be found in Ethier and Kurtz (1986).

Theorem 38 (i) If $L$ is the infinitesimal generator of a strongly continuous semigroup $\left\{P_{t}\right\}$, then $L$ is closed, $\mathcal{D}(L)$ is dense and, for any $q>0, \mathcal{R}(q I-L)=\mathbb{B}, q I-L$ is one-to-one, $\left\|(q I-L)^{-1}\right\| \leq 1 / q$, and

$$
(q I-L)^{-1} f=\int_{0}^{\infty} e^{-q t} P_{t} f d t \quad \text { for } f \in \mathbb{B}
$$

(ii) The infinitesimal generator determines the semigroup. That is, two strongly continuous contraction semigroups coincide if their infinitesimal generators coincide.

(iii) If a linear operator $L$ in $\mathbb{B}$ has a dense domain $\mathcal{D}(L)$ and, for any $q>0, \mathcal{R}(q I-L)=\mathbb{B}, q I-L$ is one-to-one, $\left\|(q I-L)^{-1}\right\| \leq 1 / q$, then $L$ is the infinitesimal generator of a strongly continuous semigroup on $\mathbb{B}$.

Proof. cf. Ethier and Kurtz (1986)

\subsubsection{Infinitesimal generators of LIBOR additive processes}

Let $C_{0}=C_{0}\left(\mathbb{R}^{d}\right)$ be the real Banach space of continuous functions $f$ from $\mathbb{R}^{d}$ into $\mathbb{R}$ satisfying $\lim _{|x| \rightarrow \infty} f(x)=0$ with norm $\|f\|=\sup _{x}|f(x)|$. Let $C_{0}^{n}$ be the set of $f \in C_{0}$ such that $f$ is $n$ times differentiable and the partial derivatives of $f$ with order $\leq n$ belong to $C_{0}$.

Suppose now that $\left\{G_{t}\right\}$ is a LIBOR additive process on $\mathbb{R}^{d}$ and the transition function $\mathbb{P}_{0, t}\left(g_{0}, B\right)$ is defined by

$$
\mathbb{P}_{0, t}\left(g_{0}, B\right):=\mu_{t}\left(B-g_{0}\right)
$$

for $t \geq 0, g_{0} \in \mathbb{R}^{d}$ and $B \in \mathcal{B}\left(\mathbb{R}^{d}\right)$

Define, for $f \in C_{0}$,

$$
\begin{aligned}
\left(\mathbb{P}_{t} f\right)\left(g_{0}\right) & =\int_{\mathbb{R}^{d}} f(g) \mathbb{P}_{t}\left(g_{0}, d g\right) \\
& =\int_{\mathbb{R}^{d}} f\left(g_{0}+g\right) \mu_{t}(d g) \\
& =\mathbb{E}\left[f\left(g_{0}+G_{t}\right)\right]
\end{aligned}
$$

Theorem 39 The family of operators $\left\{\mathbb{P}_{t}: t \geq 0\right\}$ defined above from a LIBOR additive process $\left\{G_{t}\right\}$ on $\mathbb{R}^{d}$ is a strongly continuous semigroup on $C_{0}\left(\mathbb{R}^{d}\right)$ with norm $\left\|\mathbb{P}_{t}\right\|=1$. Let L be its infinitesimal generator. 
Then

$$
\begin{aligned}
L f(g)= & \frac{1}{2} \sum_{n, m=1}^{d}\left(a_{\eta(t)}(n, m)\right) \frac{\partial^{2} f}{\partial g_{n} \partial g_{m}}(g)+\sum_{n=1}^{d}\left(\gamma_{\eta(t)}(n)\right) \frac{\partial f}{\partial g_{n}}(g) \\
& +\int_{\mathbb{R}^{d}}\left(f(g+x)-f(g)-\sum_{n=1}^{d} x_{n} \frac{\partial f}{\partial g_{n}}(g) 1_{|x| \leq 1}(x)\right) v_{\eta(t)}(d x)
\end{aligned}
$$

for $f \in C_{0}^{2}$, where $\left\{\left(\gamma_{\eta(t)}, A_{\eta(t)}, v_{\eta(t)}\right)_{t \geq 0}\right\}$ is the generating triplet of $\left\{G_{t}\right\}$ with $t \geq 0$ and $A_{\eta(t)}=$ $\left(a_{\eta(t)}(n, m)\right)_{n, m \leq d}, \gamma_{\eta(t)}=\left(\gamma_{\eta(t)}(n)\right)_{n \leq d}$

Proof. Notice that, according with Definition 37, the infinitesimal generator of the LIBOR additive process is the Lévy infinitesimal generator and the proof in. Sato (1999) Theorem 31.5 applies here (see also in Barles, Buckdahn and Pardoux (1997) Theorem 3.4. or Nualart and Schoutens (2001) for Lévy processes, or Pardoux, Pradeilles and Rao (1997) in the no-homogenous case). 


\subsection{The Lévy-Itô decomposition of LIBOR additive processes}

In the present subsection we exhibit the canonical representation for multidimensional semimartingales, and for practical purposes, we introduce here the Lévy-Itô decomposition of sample functions. This decomposition expresses sample functions of a LIBOR additive process as a sum of two independent parts; a continuous part and a part expressible as a compensated sum of independent sums. This decomposition was conceived by Paul Lévy (1934) using a direct analysis of the paths of Lévy processes, and formulated and proved by Kiyosi Itô (1942) using many pages. However there are many proofs available in the literature.

Let us recall that we assume a stochastic basis $(\Omega, \mathbb{G}, \mathbb{P})$ equipped with the "usual" filtration $\mathbb{G}=\left(\mathcal{G}_{t}\right)_{t \in\left[0, T^{*}\right]}$ which satisfies the "usual conditions". Additionally, let us consider an auxiliary measurable space $(E, \mathcal{E})$ which we assume to be a Blackwell ${ }^{5}$ space. Further

$$
E=[0, \infty) \times\left(\mathbb{R}^{d} \backslash\{0\}\right)=[0, \infty) \times D_{0, \infty}
$$

where $D_{0, \infty}=\mathbb{R}^{d} \backslash\{0\}$. Recall that we defined a time interval $\left[0, T^{\star}\right]$ with $T^{\star}$ fixed. Also, notice that we have a predetermined collection of fixed dates $0=T_{0}<T_{1}<\ldots<T_{n}=T^{\star}$. Recall also that $\eta$ is a right-continuous function $\eta:\left[0, T_{n}\right] \rightarrow\{0,1, \ldots, n\}$ by taking $\eta(t)$ to be $\eta(t)=\sup \left\{i \geq 0: T_{i} \leq t\right\}$.

A marked point $\varepsilon$ in $E$ (usually $E=\mathbb{R}^{d}$ ) is denoted by $\varepsilon=(s, x)$ with $s \in(0, \infty)$ and $x \in D_{0, \infty}$. The Borel $\sigma$-algebra of $E$ is denoted by $\mathcal{E}=\mathcal{B}(E)$. Let us define a random measure on $\mathbb{R}_{+} \times E$ as a family $v=(v(\omega ; d t, d g): \omega \in \Omega)$ of nonnegative measures on $\left(\mathbb{R}_{+} \times E, \mathcal{R}_{+} \times \mathcal{E}\right)$ satisfying $v(\omega ;\{0\} \times E)=0$ identically. Hence, the integral of $\delta(\varepsilon)$ with respect to a measure $v$ on $E$ is written as

$$
\int_{E} \delta(\varepsilon) v(d \varepsilon)=\int_{(0, \infty) \times D_{(0, \infty)}} \delta(s, x) v(d s, d x)
$$

Now we formulate the Lévy-Itô decomposition for the LIBOR additive process as the main theorem of this subsection ${ }^{6}$.

Theorem 40 (Lévy-Itô 1) Let $G=\left\{G_{t}: t \geq 0\right\}$ be a LIBOR additive process on $\mathbb{R}^{d}$ defined on a stochastic basis $(\Omega, \mathcal{G}, \mathbb{P})$ with the system of generating triplets $\left\{\left(\gamma_{\eta(t)}(t), A_{\eta(t)}, v_{\eta(t)}\right)_{t \geq 0}\right\}$ and define the measure $\mu_{\eta(t)}$ on $E$ by $\mu((0, t] \times B)=\mu_{\eta(t)}(B)$ for $B \in \mathcal{B}\left(\mathbb{R}^{d}\right)$. Using $\Omega_{0}$ from Definition 11 of LIBOR additive process, for $B \in \mathcal{B}(E)$

$$
\mu_{\eta(t)}(B, \omega)= \begin{cases}\sharp\left\{t:\left(t, G_{t}(\omega)-G_{t-}(\omega)\right) \in B\right\} & \text { for } \omega \in \Omega_{0} \\ 0 & \text { for } \omega \notin \Omega_{0}\end{cases}
$$

Then the following holds:

(i) $\left\{\mu_{\eta(t)}(B): B \in \mathcal{B}(E)\right\}$ is an integer-valued random measure (Poisson) on $E$ and $v_{\eta(t)}$ is a predictable random measure namely the compensator of the random measure $\mu_{\eta(t)}(B)$ associated to the jumps of $G$.

\footnotetext{
${ }^{5}$ In all the sequel, $E$ will actually be $\mathbb{R}_{+}^{d}$ or $\mathbb{R}^{d}$, or at most a Polish spaces with its Borel $\sigma$-fields

${ }^{6}$ For the sake of simplicity, let us define $D_{a, b}=D(a, b]=\left\{x \in \mathbb{R}^{d}: a<|x| \leq b\right\}$ and $D_{a, \infty}=D(a, \infty]=$ $\left\{x \in \mathbb{R}^{d}: a<|x|<\infty\right\}$
} 
(ii) There is $\hat{\Omega} \in \mathcal{F}$ with $\mathbb{P}[\hat{\Omega}]=1$ such that, for any $\omega \in \hat{\Omega}$

$$
\begin{aligned}
\hat{G}_{t}(\omega)= & \lim _{\varepsilon \downarrow 0} \int_{s \in(0, t], x \in D(\varepsilon, 1]}\left\{x \mu_{\eta(t)}(d(s, x), \omega)-x v_{\eta(t)}(d(s, x))\right\} \\
& +\int_{s \in(0, t], x \in D(1, \infty)} x \mu_{\eta(t)}(d(s, x), \omega)
\end{aligned}
$$

is defined for all $t \in[0, \infty)$ and the convergence is uniform in $t$ on any bounded interval. The process $\left\{G_{t}^{1}\right\}$ is a LIBOR additive process on $\mathbb{R}^{d}$ with $\left\{\left(0, v_{\eta(t)}, 0\right)\right\}$ as the system of generating triplets.

(iii) Define

$$
\tilde{G}_{t}(\omega)=G_{t}(\omega)-\hat{G}_{t}(\omega) \quad \text { for } \omega \in \Omega_{1}
$$

There is $\tilde{\Omega} \in \mathcal{F}$ with $\mathbb{P}[\tilde{\Omega}]=1$ such that, for any $\omega \in \tilde{\Omega}, \tilde{G}_{t}^{2}(\omega)$ is continuous in $t$. The process $\left\{\tilde{G}_{t}^{2}\right\}$ is a LIBOR additive process on $\mathbb{R}^{d}$ with $\left\{\left(\gamma_{\eta(t)}(t), A_{\eta(t)}, 0\right)_{t \geq 0}\right\}$ as a system of generating triplets

(iv) The two processes $\left\{\hat{G}_{t}\right\}$ (jump part) and $\left\{\tilde{G}_{t}\right\}$ (continuous part) are independent.

Proof. cf. Sato (1999) Section 20

Theorem 41 (Lévy-Itô 2) Suppose that the LIBOR additive process $G=\left\{G_{t}: t \geq 0\right\}$ in the last Theorem satisfies $\int_{|x| \leq 1}|x| v_{\eta(t)}(d x)<\infty$ for all $t>0$. Let $\gamma_{0}(t)$ be the drift of $G_{t}$. Then, there is $\hat{\Omega} \in \mathcal{F}$ with $\mathbb{P}[\hat{\Omega}]=1$ such that, for any $\omega \in \hat{\Omega}$

$$
\hat{G}_{t}(\omega)=\int_{(0, t] \times D(0, \infty)} x \mu_{\eta(t)}(d(s, x), \omega)
$$

is defined for all $t \geq 0$. The process $\left\{\hat{G}_{t}\right\}$ is a LIBOR additive process on $\mathbb{R}^{d}$ such that

$$
\mathbb{E}\left[e^{i\left\langle z, \hat{G}_{t}\right\rangle}\right]=\exp \left[\int_{\mathbb{R}^{d}}\left(e^{i\langle z, x\rangle}-1\right) v_{\eta(t)}(d x)\right]
$$

Define

$$
\tilde{G}_{t}(\omega)=G_{t}(\omega)-\hat{G}_{t}(\omega) \quad \text { for } \omega \in \Omega_{3}
$$

Then, for any $\omega \in \tilde{\Omega} \cap \hat{\Omega}, \tilde{G}_{t}(\omega)$ is continuous in $t$ and $\left\{\tilde{G}_{t}\right\}$ is an LIBOR additive process on $\mathbb{R}^{d}$ such that

$$
\mathbb{E}\left[e^{i\left\langle z, \tilde{G}_{t}\right\rangle}\right]=\exp \left[-\frac{1}{2}\left\langle z, A_{\eta(t)} z\right\rangle+i\left\langle\gamma_{\eta(t)}(t), z\right\rangle\right]
$$

The two processes $\left\{\hat{G}_{t}\right\}$ (jump part) and $\left\{\tilde{G}_{t}\right\}$ (continuous part) are independent.

Proof. cf. Sato (1999) Section 20

Theorems 40 and 41 are called the Lévy-Itô decomposition for the LIBOR additive process.

As we have already mentioned, several proofs of the Lévy-Itô theorem exist and they are very well known in the literature, even for additive processes (Sato (1999)). The simplest proof begins, first, with the construction of the Poisson random measures. Second, given any LIBOR additive process $\left\{G_{t}\right\}_{t \geq 0}$ 
we use its system of generating triplets $\left\{\left(\gamma_{\eta(t)}(t), A_{\eta(t)}, v_{\eta(t)}\right)_{t \geq 0}\right\}$ in order to construct an additive process $\left\{Y_{t}\right\}_{t \geq 0}$ such that $\left\{Y_{t}\right\}_{t \geq 0} \stackrel{d}{=}\left\{G_{t}\right\}_{t \geq 0}$ and $\left\{Y_{t}\right\}_{t \geq 0}$ has the Lévy-Itô decomposition. Third, using the facts that $\left\{G_{t}\right\}_{t>0}$ and $\left\{Y_{t}\right\}_{t>0}$ induce an identical probability measure on the Skorohod space $\mathbb{D}=\mathbb{D}\left([0, \infty), \mathbb{R}^{d}\right)$ of right continuous paths with left limits with the $\sigma$-algebra $\mathcal{F}_{D}$ generated by the Borel cylinder sets and that all relevant quantities are $\mathcal{F}_{D}-$ measurable, we can prove that $\left\{G_{t}\right\}_{t \geq 0}$ also has the Lévy-Itô decomposition. 


\subsection{Applications to sample-function properties: continuity, jump- ing times and increasingness}

From the Lévy-Itô decomposition we can deduce many sample function properties of LIBOR additive processes. Following Sato (1999), we devote this subsection to studying fundamental properties of sample functions of processes with independent increments and piecewise stationarity, such as continuity, jumping times, and increasingness.

Theorem 42 (Continuity) Sample functions of $\left\{G_{t}\right\}_{t \geq 0}$ are continuous a.s. if and only if $v_{\eta(t)}=0$ for every $t \in\left[0, T^{\star}\right]$

Proof. By Theorem 40 (Lévy-Itô 1) the number of jumping times satisfying $\left|G_{t}-G_{t_{-}}\right| \in D_{\varepsilon, \infty}$ has mean $t \int_{|g|>\varepsilon} v_{\eta_{(t)}}(d g)$. Hence the number of jumps is 0 a.s. if and only if $v_{\eta_{(s)}}=0$ for every $s \in[0, t]$.

Theorem 43 (Jumping times) If $v_{\eta(t)}\left(\mathbb{R}^{d}\right)=\infty$ for every $t \in\left[0, T^{\star}\right]$, then, almost surely, jumping times are countable and dense in $[0, \infty)$. If $0<v_{\eta(t)}\left(\mathbb{R}^{d}\right)<\infty$ for every $t \in\left[0, T^{\star}\right]$ then, almost surely, jumping times are infinitely many and countable in increasing order, and the first jumping time $T(\omega)$ has exponential distribution with mean $1 / v_{\eta(t)}\left(\mathbb{R}^{d}\right)$.

Proof. Countability of jumps is a consequence of right-continuity with left-limits. For $\varepsilon>0$ and $\omega \in \Omega_{0}$ let $T_{\varepsilon}(\omega)$ be the first time that $G_{t}(\omega)$ jumps with size $>\varepsilon$. Let $T_{\varepsilon}(\omega)=\infty$ if $G_{t}(\omega)$ does not have any jump with size $>\varepsilon$. Since $T_{\varepsilon}(\omega) \leq t$ is equivalent to if $\int_{(0, t] \times(\varepsilon, \infty)} \mu_{\eta(t)}(d(s, z), \omega) \geq 1$,

$$
\mathbb{P}\left[T_{\varepsilon} \leq t\right]=1-\exp \left[-t \int_{D_{\varepsilon, \infty}} v_{\eta(t)}(d g)\right]
$$

by Theorem 40 (Lévy-Itô 1 ). Hence, if $\int_{D_{\varepsilon, \infty}} v_{\eta(t)}(d g)=c>0$, then $T_{\varepsilon}$ has exponential distribution with mean $1 / c$.

Suppose that $v_{\eta(t)}\left(\mathbb{R}^{d}\right)=\infty$ for every $t \in\left[0, T^{\star}\right]$, then $\lim _{\varepsilon \downarrow 0} P\left[T_{\varepsilon} \leq t\right]=1$ for any $t>0$, and hence $\lim _{\varepsilon \downarrow 0} T_{\varepsilon}=0$ a.s. Hence there is $E_{0} \in \mathcal{F}$ with $P\left[E_{0}\right]=1$ such that for any $\omega \in E_{0}$ the time 0 is a limiting point of jumping times of $G_{t}(\omega)$, and for any $s>0$ there is $E_{s} \in \mathcal{F}$ with $P\left[E_{s}\right]=1$ such that for any $\omega \in E_{s}$ the set of jumping times $s$ as a limiting point from the right. Consider $E=\cap_{s \in \mathbb{Q}^{+}} E_{s}$. Jumping times are dense in $[0, \infty)$ for any $\omega \in E$.

Now, suppose that $0<v_{\eta(t)}\left(\mathbb{R}^{d}\right)<\infty$ for every $t \in\left[0, T^{\star}\right]$. By Theorem 40 (Lévy-Itô 1$), \mu_{\eta(t)}$ has a Poisson distribution with mean $t v_{\eta(t)}\left(\mathbb{R}^{d}\right)$ and $\mu_{\eta(t)}<\infty$ a.s. Hence the jumping times are enumerable in increasing order. The first jumping time $T$ has exponential distribution with mean $1 / v_{\eta(t)}\left(\mathbb{R}^{d}\right)$ because $P[T \leq t]=P[\mu(t) \geq 1]=1-e^{-t v_{\eta(t)}\left(\mathbb{R}^{d}\right)}$. It follows that $T(\omega)<\infty$ a.s. Let $T^{(s)}$ be the first jumping time after $s$, hence $T^{(s)}<\infty$ a.s. Hence there are infinitely many jumps, a.s.

Notice that in the case of $0<v_{\eta(t)}\left(\mathbb{R}^{d}\right)<\infty$ we can actually say more: if we denote the nth jumping time by $U_{n}(\omega)$ and $U_{0}(\omega)=0$, then $\left\{U_{n}-U_{n-1}: n \in \mathbb{N}\right\}$ constitutes independent identically distributed random variables, each distributed with mean $1 / v_{\eta(t)}\left(\mathbb{R}^{d}\right)$ and $\lim _{n \rightarrow \infty} U_{n}(\omega)=\infty$ a.s. To see this note that $\left\{\mu_{\eta(t)}\right\}$ is a Poisson process with parameter $v_{\eta(t)}\left(\mathbb{R}^{d}\right)$.

Definition 44 A LIBOR additive process $\left\{Z_{t}\right\}_{t \geq 0}$ is said to be increasing if $Z_{t}(\omega)$ is increasing as a function of $t$, a.s. 
Theorem 45 (Increasingness) Let $d=1$. A LIBOR additive process $\left\{Z_{t}\right\}_{t \geq 0}$ on $\mathbb{R}$ is increasing if and only if $A_{\eta(t)}=0, \quad \int_{(-\infty, 0)} v_{\eta(t)}(d z)=0, \quad \int_{(0, \infty)} z \cdot v_{\eta(t)}(d z)<\infty$ and $\gamma_{\eta(t)} \geq 0$, for every $t \in\left[0, T^{\star}\right]$.

Proof. The 'only if' part follows from the fact that $\int_{(-\infty, 0)} v_{\eta(t)}(d z)=0$ implies $\mu_{\eta(t)}((0, t] \times(-\infty, 0))=$ 0 and therefore $\left\{Z_{t}\right\}_{t \geq 0}$ does not have negative jumps. Hence, by the last theorem we have that

$$
Z_{t}=t \cdot \gamma_{\eta(t)}+\int_{(0, t] \times(0, \infty)} z \cdot \mu_{\eta(t)}(d(s, z)) \text { a.s. for every } t \in\left[0, T^{\star}\right]
$$

because the continuous part $\tilde{Z}_{t}=t \cdot \gamma_{\eta(t)}$ and this shows that $\left\{Z_{t}\right\}_{t \geq 0}$ is increasing.

And the 'if' part follows from the fact that since $\left\{Z_{t}\right\}_{t \geq 0}$ has no negative jumps, we have that $v_{\eta(t)}((-\infty, 0))=0$ for every $t \in\left[0, T^{\star}\right]$. Since an increasing function remains increasing after a finite number of its jumps are deleted, we have $Z(t)-Z_{\varepsilon}(t) \geq 0$, hence

$$
\begin{aligned}
Z^{\prime}(t) & =\lim _{\varepsilon \downarrow 0} Z_{\varepsilon}(t) \\
& =\int_{(0, t] \times(0, \infty)} z \cdot \mu_{\eta(t)}(d(s, z))
\end{aligned}
$$

exists and is bounded above by $Z(t)$.

Hence, we have that the generating function or Laplace transform of its distribution is

$$
\begin{aligned}
\mathbb{E}\left[e^{-u Z_{\varepsilon}(t)}\right] & =\exp \left[t \int_{(\varepsilon, \infty)}\left(e^{-u z}-1\right) \cdot v_{\eta(t)}(d z)\right] \\
& =\exp \left[t \int_{(\varepsilon, \infty)}\left(e^{-u z}-1+u z 1_{(0,1]}(z)\right) \cdot v_{\eta(t)}(d z)-t u \int_{(\varepsilon, 1]} z \cdot v_{\eta(t)}(d z)\right]
\end{aligned}
$$

for $u>0$.

As $\varepsilon \downarrow 0, \mathbb{E}\left[e^{-u Z_{\varepsilon}(t)}\right]$ tends to $\mathbb{E}\left[e^{-u Z^{\prime}(t)}\right]$ which is positive, and

$$
\int_{(\varepsilon, \infty)}\left(e^{-u z}-1+u z 1_{(0,1]}(z)\right) \cdot v_{\eta(t)}(d z)
$$

tends to the integral over $(0, \infty)$ which is finite.

Hence, we have that $\int_{(0, \infty)} z \cdot v_{\eta(t)}(d z)<\infty$, and directly by application of Theorem 41 (Lévy-Itô 2) we have that $Z(t)=\hat{Z}(t)+\tilde{Z}(t)$, where the jump part $\hat{Z}(t)=Z^{\prime}(t)$ and the continuous part $\tilde{Z}(t)$ has the generating system of triplets $\left(A_{\eta(t)}, 0, \gamma_{\eta(t)}\right)$. But $\tilde{Z}(t)=Z(t)-Z^{\prime}(t) \geq 0$ and therefore $A_{\eta(t)}=0$ and $\gamma_{\eta(t)} \geq 0$ for every $t \in\left[0, T^{\star}\right]$.

According to the last theorem, notice that a LIBOR additive process on $\mathbb{R}$ generated by $\left\{\left(\gamma_{\eta(t)}(t), A_{\eta(t)}, v_{\eta(t)}\right)\right\}$ with $A_{\eta(t)}=0, v_{\eta(t)}((-\infty, 0))=0$ and $\int_{(0,1]} z \cdot v_{\eta(t)}(d z)=\infty$ for every $t \in\left[0, T^{\star}\right]$, has positive jumps only, does not have Brownian-like part, but it is fluctuating, not increasing, no matter how large $\gamma$ is. An explanation is that such a process can exist only with infinitely strong drift in the negative direction, which cancels the divergence of the sum of jumps; but it causes a random continuous motion in the negative direction. It is clear that an increasing LIBOR additive process will not have negative jumps, but also the drift has to be positive or zero, for every interval in $I$. 


\subsection{Density Transformations of LIBOR additive processes}

In this subsection we cite the most important results from Jacod and Shiryaev (1987) Chapter III, Bjork, Kabanov and Runggaldier (1997) and Sato (1999) Chapter 6, concerning Girsanov's theorem and the explicit computation of density processes of absolutely continuous probability measures. This subsection mainly serves the purpose of preparation for the usual change of measure for financial models driven by LIBOR additive processes.

Roughly speaking, the basic idea is the following. Let $G$ be a semimartingale on some stochastic basis $(\Omega, \mathcal{F}, \mathbb{P})$. Then it is well known that the class of semimartingales is invariant with respect to equivalent transformation of measure, or in other words, $G$ remains a semimartingale on $(\Omega, \mathcal{F}, \mathbb{Q}$ ) (see Rogers and Williams (1987) IV.38) where $\mathbb{Q}$ is locally absolutely continuous to $\mathbb{P}$. This change of measure can be described by two sequences $\beta_{i}$ and $Y_{i}$ (we will give an explicit expression later) called Girsanov quantities, in the sense that the density process $Z$ of $\mathbb{Q}$ with respect to $\mathbb{P}$ can be expressed via $\beta_{i}$ and $Y_{i}$.

As usual, we assume that two measures $\mathbb{P}$ and $\mathbb{Q}$ on a common measurable space $(\Omega, \mathbb{G})$ are called mutually absolutely continuous or equivalent measures, written $\mathbb{P} \approx \mathbb{Q}$ if the collection $\left\{B \in \mathcal{G}_{t}: \mathbb{P}(B)=0\right\}$ is identical with $\left\{B \in \mathcal{G}_{t}: \mathbb{Q}(B)=0\right\}$. The Radon-Nikodym derivative of $\mathbb{Q}$ with respect to $\mathbb{P}$ is denoted by $\frac{d \mathbb{Q}}{d \mathbb{P}}$. If $\mathbb{P} \approx \mathbb{Q}$ then $\frac{d \mathbb{Q}}{d \mathbb{P}}$ is positive and finite $\mathbb{P}$-almost everywhere.

Let us start with the following useful theorems about stochastic exponentials, and nice-versions of triplets characteristics for the LIBOR additive process.

Theorem 46 Let $G=\left\{G_{t}: t \geq 0\right\}$ be a LIBOR additive process and consider the stochastic differential equation

$$
d Z=Z_{-} d G, \quad Z_{0}=1
$$

This equation has a unique (up to indistinguishability) "càdlàg" adapted solution, called the stochastic exponential of $G$, which is a semimartingale and is denoted by $\mathcal{E}(G)$. Explicitly

$$
\mathcal{E}(G)_{t}=\exp \left(G_{t}-\frac{1}{2}\langle\tilde{G}\rangle_{t}\right) \prod_{s \leq t}\left(1+\hat{G}_{s}\right) e^{-\hat{G}_{s}}
$$

where $\tilde{G}_{t}$ is the continuous part in $t$, and $\hat{G}_{t}$ is the jump part of $G_{t}$. If we define $\tau:=\inf \left\{t \geq 0: \hat{G}_{t}=-1\right\}$ then $\mathcal{E}(G) \neq 0$ on $[0, \tau)$, and $\mathcal{E}(G)=0$ on $[\tau, \infty)$.

Proof. cf. Jacod and Shiryaev (1987) Theorem I.4.61

Theorem 47 Let $G=\left\{G_{t}: t \geq 0\right\}$ be a LIBOR additive process on $\mathbb{R}^{d}$. Then there exist a "niceversion" of the triplet characteristics for $G$ which is of the form

$$
\left\{\begin{array}{l}
A_{\eta_{(t)}}=\left(A_{\eta_{(t)}},(i, j)\right)_{i, j \leq d}=\sum_{j \leq \eta(t)} \int_{T_{j-1}}^{T_{j} \wedge t} \hat{a}_{\eta_{(t)}}(i, j) \cdot d H_{\eta_{(t)}}(\omega, t) \\
v_{\eta_{(t)}}(\omega ; d t, d g)=K_{\omega, t}(d g) \cdot d H_{\eta_{(t)}}(\omega) \\
\gamma_{\eta_{(t)},(i)}=\sum_{j \leq \eta(t)} \int_{T_{j-1} \wedge t}^{T_{j} \wedge t} b_{\eta_{(t)}}(i) \cdot d H_{\eta_{(t)}}(\omega, t)
\end{array}\right.
$$

where $H_{\eta_{(t)}}$ is a real-valued predictable, increasing and locally integrable process, $\hat{\gamma}_{\eta_{(t)}}(t)=\left(b_{\eta_{(t)}}(i)\right)_{1 \leq i \leq d}$ is an $\mathbb{R}^{d}$-valued predictable process, $\hat{A}_{\eta_{(t)}}=\left(\hat{a}_{\eta_{(t)}}(i, j)\right)_{1 \leq i, j \leq d}$ is a predictable process with values in the set of all symmetric nonnegative definite $d \times d$ matrices, and $K_{\omega, t}(d g)$ is a transition kernel from 
$\left(\Omega \times \mathbb{R}_{+}, \mathbb{P}\right)$ into $\left(\mathbb{R}^{d}, \mathcal{B}^{d}\right)$ which satisfies for any $t \in\left[0, T^{\star}\right]$

$$
K_{\omega, t}(\{0\})=0 \text { and } \int_{\mathbb{R}^{d}}\left(|g|^{2} \wedge 1\right) K_{\omega, t}(d g) \leq 1
$$

Proof. cf. Jacod and Shiryaev (1987) Proposition II.2.9.

Theorem 48 (Girsanov's theorem for semimartingales) Assume that $\mathbb{P}$ and $\mathbb{Q}$ are (locally) equivalent measures, and let $G=\left\{G_{t}: t \geq 0\right\}$ be a LIBOR additive process (piecewise homogeneous semimartingale) with $\mathbb{P}$-characteristics $\left.\left(A_{\eta_{(t)}}, \nu_{\eta_{(t)}}, \gamma_{\eta_{(t)}}(t)\right)\right|_{\mathbb{P}}$. Let $\hat{a}_{\eta_{(t)}}$ and $H_{\eta_{(t)}}$ be the processes of the "nice-version" from Theorem 47 for every $\eta_{(t)}=0,1, \ldots, n$ and $t \in\left[0, T^{\star}\right]$, and let $\mathbb{P} \approx \mathbb{Q}$. Then there exists a sequence $\left(Y_{\eta_{(t)}}, \beta_{\eta_{(t)}}\right)$ where $Y_{\eta_{(t)}}$ is a $\mathbb{P}$-measurable nonnegative function and $\beta_{\eta_{(t)}}=\left(\beta_{\eta_{(t)}}(i)\right)_{i \leq d}$ is a sequence of predictable processes, for any $t \in\left[0, T^{\star}\right]$ satisfying

$$
\begin{aligned}
& \int\left(Y_{\eta_{(t)}}-1\right) c(g) d v_{\eta_{(t)}}^{\mathbb{P}}<\infty \\
& \sum_{j \leq \eta(t)} \int_{T_{j-1} \wedge}^{T_{j} \wedge t}\left|\hat{a}_{\eta_{(t)}} \beta_{\eta_{(t)}}\right| d H_{\eta_{(t)}}<\infty \\
& \sum_{j \leq \eta(t)} \int_{T_{j-1}}^{T_{j} \wedge t}\left(\beta_{\eta_{(t)}}^{\prime} \hat{a}_{\eta_{(t)}} \beta_{\eta_{(t)}}\right) d H_{\eta_{(t)}}<\infty
\end{aligned}
$$

$\mathbb{Q}$-a.s. for any $t \in\left[0, T^{\star}\right]$ and such that a version of the characteristics of $G$ relative to $\mathbb{Q}$ are $\left(A_{\eta_{(t)}}^{\mathbb{Q}}, \nu_{\eta_{(t)}}^{\mathbb{Q}}, \gamma_{\eta_{(t)}}^{\mathbb{Q}}(t)\right)$, such that

$$
\left\{\begin{array}{l}
A_{\eta_{(t)}}^{\mathbb{Q}}=A_{\eta_{(t)}}^{\mathbb{P}} \\
v_{\eta_{(t)}}^{\mathbb{Q}}=Y_{\eta_{(t)}}^{\mathbb{P}} \cdot v_{\eta_{(t)}}^{\mathbb{P}} \\
\gamma_{\eta_{(t)}}^{\mathbb{Q}}(t)=\gamma_{\eta_{(t)}}^{\mathbb{P}}+\sum_{j \leq \eta(t)} \int_{T_{j-1}}^{T_{j} \wedge t}\left|\hat{a}_{j} \beta_{j}\right| d H_{j}+\int_{\mathbb{R}}\left(Y_{\eta_{(t)}}-1\right) c(g) v_{\eta_{(t)}}^{\mathbb{P}}(d g)
\end{array}\right.
$$

Proof. cf. Jacod and Shiryaev (1987) Theorem III.3.24

Definition 49 The quantities $\beta_{j}$ and $Y_{j}$ for any $j=0, \ldots, \eta(t)$ from Theorem 48 are called Girsanov quantities of $\mathbb{Q}$ with respect to $\mathbb{P}$ relative to $G$, or simply Girsanov quantities of $\mathbb{Q}$.

Remark 50 Notice that the $Y_{\eta_{(t)}}$ describe how the jump distribution of $G$ change when we pass from $\mathbb{P}$ to $\mathbb{Q}$, and $\beta_{\eta_{(t)}}$ together with $Y_{\eta_{(t)}}$ determines the changes in drift. On the other hand, notice that the Girsanov quantities are not unique: from the uniqueness of $v_{\eta_{(t)}}^{\mathbb{P}}$ and $v_{\eta_{(t)}}^{\mathbb{Q}}$ we only get uniqueness of $Y_{\eta_{(t)}}$ on supp $v_{\eta_{(t)}}^{\mathbb{P}}$. And with the uniqueness of $\gamma_{\eta_{(t)}}^{\mathbb{Q}}(t)$ and $\gamma_{\eta_{(t)}}^{\mathbb{P}}$ we only get the uniqueness of $\hat{a}_{\eta_{(t)}} \beta_{\eta_{(t)}}$ for fixed $\hat{a}_{\eta_{(t)}}$, for any $t \in\left[0, T^{\star}\right]$.

Example 51 Let $W$ be a standard $\mathbb{R}^{d}$-valued $\mathbb{P}$-Brownian motion and let $A$ be a $d \times d$ matrix. Set $G:=A \cdot W$ and take $\mathbb{P} \approx \mathbb{Q}$ with Girsanov quantities $\beta$ and $Y$ relative to $G$. Then $\tilde{G}=G$, so $\langle G\rangle_{t}=A A^{\prime} t$, and the $\mathbb{P}$-characteristic triplet of $G$ are given by

$$
\begin{aligned}
A_{t}^{\mathbb{P}} & =a t \\
\nu_{t}^{\mathbb{P}} & =0 \\
\gamma^{\mathbb{P}}(t) & =0
\end{aligned}
$$


where $a:=A A^{\prime}$ is the covariance matrix of $G$. By Theorem 48 we get the $\mathbb{Q}$-characteristic triplet of $G$ by

$$
\begin{aligned}
A_{t}^{\mathbb{Q}} & =a t \\
\nu_{t}^{\mathbb{Q}} & =0 \\
\gamma^{\mathbb{Q}}(t) & =\int_{0}^{t}\left(a \beta_{s}\right) d s
\end{aligned}
$$

Note that $G$ remains a Lévy process under $\mathbb{Q}$ if and only if $\beta$ is deterministic and independent of time. In this case, $G$ is a linear transformation of a standard Brownian motion with constant drift, i.e. $G_{t}=$ $A W_{t}^{\mathbb{Q}}+a \beta t$. 


\subsection{Examples of LIBOR additive processes}

In the following sections we briefly list a number of popular processes that can be studied as special cases of the LIBOR additive processes or non-homogeneous Lévy processes. We pay special attention to their density function, their characteristic function, their characteristic triplet together with some other properties.

\subsubsection{The non-homogeneous Poisson Process}

Given a tenor structure $0=T_{0}<T_{1} \ldots<T_{n}=T^{\star}$, the non-homogeneous Poisson process is the simplest LIBOR additive process we can think of. It is based on the Poisson $\left(\lambda_{j}\right)_{j=1 \ldots n}$ distribution which has

$$
\hat{\mu}_{j}(z)=\exp \left[\lambda_{j}(\exp [i z]-1)\right]
$$

as a characteristic function for any $j=0,1, \ldots, n$.

Like an ordinary Poisson process it has independent increments and these increments are Poisson distributed, but increments over different intervals of equal length can have different means. In particular, the number of jumps in an interval $\left(T_{j}, T_{j+1}\right]$ has a Poisson distribution with mean $\Lambda\left(T_{j+1}\right)-\Lambda\left(T_{j}\right)$ where

$$
\Lambda(t)=\sum_{j \leq \eta(t)} \int_{T_{j}}^{t \wedge T_{j+1}} \lambda_{j}(s) d s
$$

Therefore, the Poisson distribution lives on the nonnegative integers $\{0,1,2, \ldots\}$, such that

$$
\mathbb{P}\left(G_{t}=g\right)=\frac{e^{-\Lambda(t)} \Lambda(t)^{g}}{g !}
$$

Since the in-homogeneous Poisson $\left(\mu_{j}\right)_{j=1 \ldots n}$ distribution is infinitely divisible we can define an inhomogeneous Poisson process as the process that starts at zero, has independent increments property, is stochastically continuous and has piecewise stationary increments. The in-homogeneous Poisson process turns out to be an increasing pure jump process, with jump sizes always equal to 1 . This means that the additive process triplet is given by

$$
\left[0,0, \lambda_{\eta(t)} \delta(1)\right]
$$

where $\delta(1)$ denotes the Dirac measure at point 1.

\subsubsection{The non-homogeneous Compound Poisson Process}

Let $N=\left\{N_{t}, t \geq 0\right\}$ be a non-homogeneous Poisson process with intensity parameters $\left(\lambda_{j}\right)_{j=1 \ldots n}$ and let $J_{k}$ be independent and identically distributed (i.i.d.) random variables independent of $N$ and following a law, $\mu_{J}$ say, with characteristic function $\hat{\mu}_{J}(z)$. Then we say that $G=\left\{G_{t}: t \geq 0\right\}$ is a non-homogeneous Compound Poisson process if

$$
G_{t}=\sum_{k=1}^{N_{t}} J_{k}, t \geq 0
$$

The value of the process at time $t, G_{t}$, is a sum of $N_{t}$ random numbers with law $\mu_{J}$. Notice that the ordinary non-homogeneous Poisson process corresponds to the case where $J_{k}, k=1,2, \ldots$ i.e. have law $\mu_{J}$ degenerate at the point 1. 
Let us write, for a Borel set $A$, the distribution function of the law $\mu_{J}$ as follows

$$
\mathbb{P}\left(J_{k} \in A\right)_{t}=\frac{v_{\eta(t)}(A)}{\lambda_{\eta(t)}}
$$

where $v_{\eta(t)}(\mathbb{R})=\lambda_{\eta(t)}<\infty$ with $v_{\eta(t)}(\{0\})=0$. Then the characteristic function of $G_{t}$ is given by

$$
\begin{aligned}
\mathbb{E}\left[\exp \left(i z G_{t}\right)\right] & =\prod_{j \leq \eta(t)} \mathbb{E}\left(\exp \left[i z\left(G_{t \wedge T_{j+1}}-G_{T_{j}}\right)\right]\right) \\
& =\prod_{j \leq \eta(t)} \exp \left(\left(t \wedge T_{j+1}-T_{j}\right) \int_{\mathbb{R}}\left(e^{i z g}-1\right) v_{j}(d g)\right) \\
& =\prod_{j \leq \eta(t)} \exp \left(\left(t \wedge T_{j+1}-T_{j}\right) \lambda_{j}\left(\mu_{J}(z)-1\right)\right)
\end{aligned}
$$

From this we can easily obtain the characteristic triplet

$$
\left[\int_{-1}^{+1} g v_{\eta(t)}(d g), 0, v_{\eta(t)}(d g)\right]
$$
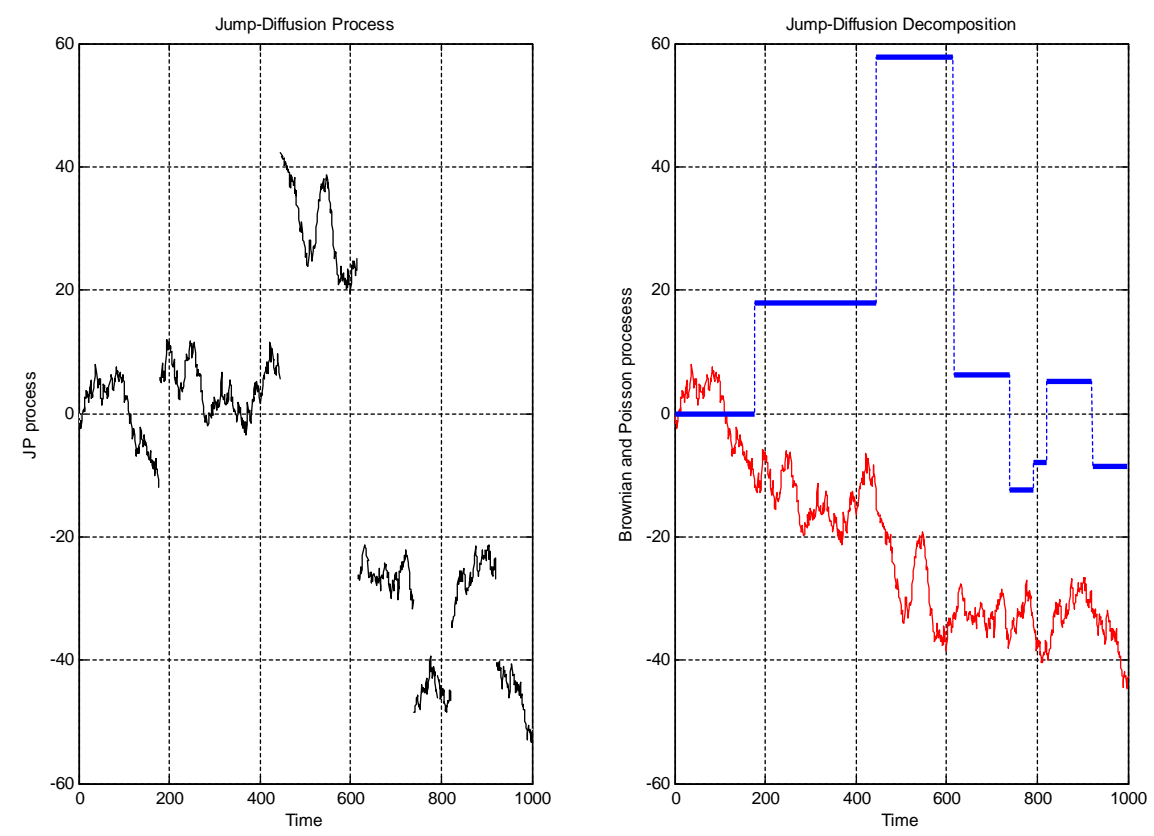

Figure (1): Sample-path of a jump-diffusion process, with the Lévy-Itò decomposition

The non-homogeneous compound Poisson process is usually introduced in the simulation of jumpdiffusion sample paths on a fixed grid is shown in Figure (1). On the left side, the figure shows us two independent sample paths, the usual continuous-part given by a Brownian motion, and the jump-part simulated using a compound Poisson process. Both together, by application of Lévy-Itô Theorem 42, form the jump-diffusion sample-path that appear in the right part of Figure (1). Efficient algorithms to simulate Poisson, exponential and Gaussian processes can be found in Press et al. (1992). Different methods of approximation of the small jumps are discussed in Schoutens (2003). 


\subsubsection{The non-homogeneous Gamma Process}

Here the non-homogeneous Gamma process $G=\left\{G_{t}: t \geq 0\right\}$ with parameters $a_{j}, b_{j}>0$ for every $j=0,1, \ldots, n$, is defined as the stochastic process which starts at zero and has independent increments, is stochastically continuous and has independent Gamma distributed increments inside of each interval for every $j=0,1, \ldots, n$.

The density function of the non-homogeneous Gamma distribution $\left(a_{j}, b_{j}\right)$ with $a_{j}>0$ and $b_{j}>0$ for every $j=0,1, \ldots, n$ is given by

$$
\mu_{j}\left(g ; a_{j}, b_{j}\right)=\frac{b_{j}^{a_{j}}}{\Gamma\left(a_{j}\right)} g^{a_{j}-1} \exp \left(-g b_{j}\right), \text { with } g>0
$$

Notice that this function has a semi-heavy (right) tail.

The characteristic function is given by

$$
\hat{\mu}_{j}\left(z ; a_{j}, b_{j}\right)=\left(1-\frac{i z}{b_{j}}\right)^{-a_{j}}
$$

and obviously, it is infinitely divisible. The characteristic triplet of a non-homogeneous Gamma process is given by

$$
\left[\frac{a_{j}\left(1-\exp \left(b_{j}\right)\right)}{b_{j}}, 0, a_{j} \frac{\exp \left(-b_{j} g\right) 1_{\{g>0\}} d g}{g}\right]
$$

The most common method of simulation for Gamma processes can be found in Marsaglia and Tsang (2000).

\subsubsection{The non-homogeneous Inverse Gaussian Process}

Let $T^{(a, b)}$ be the first time a standard Brownian motion with drift $b>0$ reaches the positive level $a>0$. It is well known that this random time follows the so-called Inverse Gaussian $I G(a, b)$ law. The $I G$ distribution is infinitely divisible. Hence we can define the $I G$ process $G=\left\{G_{t}: t \geq 0\right\}$ with parameters $a_{j}, b_{j}>0$ for any $j=0,1, \ldots, n$ as the process which starts at zero, is stochastically continuous and has independent $I G$ distributed increments, homogeneous only inside of each interval for every $j=0,1, \ldots, n$ such that for any $t \in\left[0, T^{\star}\right]$

$$
\begin{aligned}
\mathbb{E}\left(\exp \left[i z G_{t}\right]\right) & =\prod_{j \leq \eta(t)} \mathbb{E}\left(\exp \left[i z\left(G_{t \wedge T_{j+1}}-G_{T_{j}}\right)\right]\right) \\
& =\prod_{j \leq \eta(t)} \exp \left[-a_{j}\left(t \wedge T_{j+1}-T_{j}\right)\left(\sqrt{-2 i z+b_{j}^{2}}-b_{j}\right)\right]
\end{aligned}
$$

The density function of the $I G\left(a_{j}, b_{j}\right)$, for any $j=0,1, \ldots, n$ is explicitly known:

$$
\mu_{j}\left(g ; a_{j}, b_{j}\right)=\frac{a_{j}}{\sqrt{2 \pi}} \exp \left(a_{j} b_{j}\right) g^{-3 / 2} \exp \left(-\frac{1}{2}\left(\frac{a_{j}^{2}}{g}+b_{j}^{2} g\right)\right)
$$

and the Lévy measure associated to the $I G\left(a_{j}, b_{j}\right)$ law is given by

$$
v_{j}(d g)=\frac{a_{j}}{\sqrt{2 \pi}} g^{-3 / 2} \exp \left(-\frac{1}{2} b_{j}^{2} g\right) 1_{\{g>0\}} d g
$$


The first component of the characteristic triplet equals

$$
\gamma_{j}=\frac{a_{j}}{b_{j}}\left(2 \Phi\left(b_{j}\right)-1\right)
$$

where $\Phi(x)$ is the Normal distribution function.

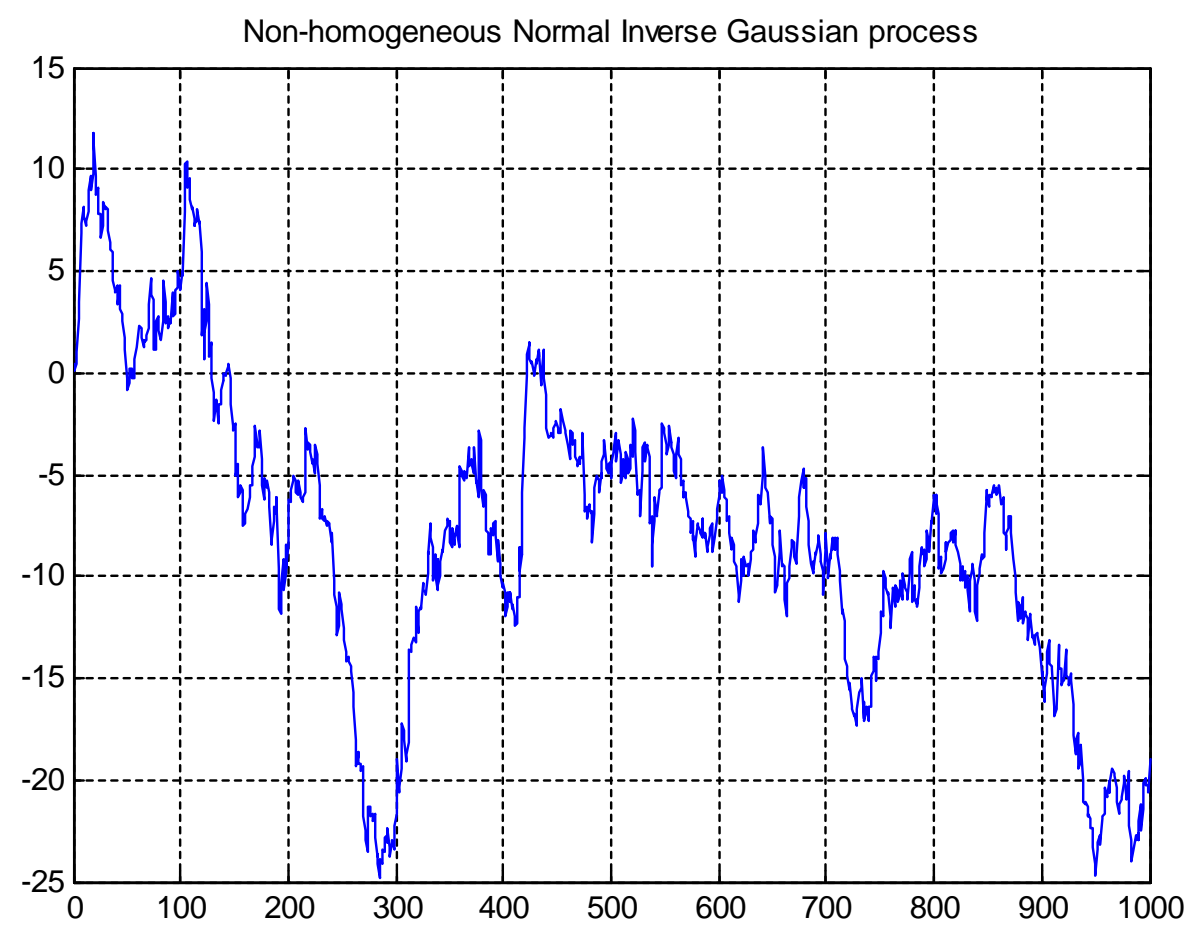

Figure (2): Non-homogeneous IG process with parameters $\left(a_{j}, 0\right)$.

Figure (2) represents a non-homogeneous inverse Gaussian process with parameters $\left(a_{j}, 0\right)$ where $a_{j}$ goes from $a_{0}=1$ to $a_{1000}=2$. Simulation algorithm can be found in Prause (1997).

\subsubsection{The non-homogeneous Generalized Inverse Gaussian Process}

The Inverse Gaussian $I G(a, b)$ law can be generalized to what is called the Generalized Inverse Gaussian distribution $G I G(a, b)$. This distribution on the positive half-line is given in terms of its density function

$$
\mu(g ; \lambda, a, b)=\frac{(b / a)^{\lambda}}{2 K_{\lambda}(a b)} g^{\lambda-1} \exp \left(-\frac{1}{2}\left(\frac{a^{2}}{g}+b^{2} g\right)\right)
$$

The parameters $\lambda, a$ and $b$ are such that $\lambda \in \mathbb{R}$ while $a$ and $b$ are both nonnegative and not simultaneously 0 .

The characteristic function is given by

$$
\hat{\mu}(k ; \lambda, a, b)=\frac{1}{K_{\lambda}(a b)}\left(1-2 i k / b^{2}\right)^{\lambda / 2} K_{\lambda}\left(a b \sqrt{1-2 i k / b^{-2}}\right)
$$

where $K_{\lambda}(g)$ denotes the modified Bessel function with the index $\lambda$. 
Hence we can define the non-homogeneous $G I G$ process as a stochastic process that starts at zero, is continuous in probability with independent increments, and is piecewise stationary or stationary inside each interval $\left[T_{j}, T_{j+1}\right]$ for $j=0,1, \ldots, n$. According to Barndorff-Nielsen and Shephard (2001), it has an infinitely divisible distribution with the following Lévy measure

$$
v_{j}(d g)=\frac{\exp \left(-\frac{1}{2} b_{j} g\right)}{g}\left(a_{j}^{2} \int_{0}^{\infty} \exp (-g z) h_{j}(z) d z+\max \left\{0, \lambda_{j}\right\}\right) d g
$$

where

$$
h_{j}(z)=\left(\pi^{2} a_{j} z\left(J_{|\lambda|}^{2}\left(a_{j} \sqrt{2 z}\right)+N_{|\lambda|}^{2}\left(a_{j} \sqrt{2 z}\right)\right)\right)^{-1}
$$

and where $J$ and $N$ are Bessel functions. Simulation algorithm can be found in Prause (1997).

\subsubsection{The non-homogeneous $\alpha$-Stable Process}

Since Mandelbrot (1963) introduced the $\alpha$-stable distribution to model the empirical distribution of asset prices, the $\alpha$-stable distribution became a popular alternative to the normal distribution which has been rejected by numerous empirical studies that have found financial return series to be heavy-tailed and possibly skewed.

More explicitly, we can define the non-homogeneous $\alpha$-stable process as a stochastic process that starts at zero, is continuous in probability with independent increments, and is piecewise stationary or stationary inside each interval $\left[T_{j}, T_{j+1}\right]$ for $j=0,1, \ldots, n$ with a Lévy measure of the form

$$
v_{j}(d g)=\frac{A}{g^{\alpha_{j}+1}} 1_{\{g>0\}} d g+\frac{B}{g^{\alpha_{j}+1}} 1_{\{g<0\}} d g
$$

for some positive constants $A$ and $B^{7}$. The characteristic function of a real-valued non-homogeneous stable random variable $G$ has the form

$$
\hat{\mu}_{j}(z)= \begin{cases}\exp \left\{-\sigma_{j}^{\alpha_{j}}|z|^{\alpha_{j}}\left(1-i \beta_{j} \operatorname{sgn} z \tan \frac{\pi \alpha_{j}}{2}\right)+i \theta z\right\}, & \text { if } \alpha \neq 1 \\ \exp \left\{-\sigma_{j}|z|\left(1+i \beta_{j} \frac{2}{\pi} \operatorname{sgn} z \log |z|\right)+i \theta z\right\}, & \text { if } \alpha=1\end{cases}
$$

where $\alpha_{j} \in(0,2], \sigma_{j} \geq 0, \beta_{j} \in[-1,1]$ and $\theta \in \mathbb{R}$,

\footnotetext{
${ }^{7}$ Note the link between between $\alpha$-stable processes and TS processes. A tempered stable process (TS) is usually obtained by taking a one-dimensional stable process and multiplying the Lévy measure with a decreasing exponential on each half of the real axis.
} 


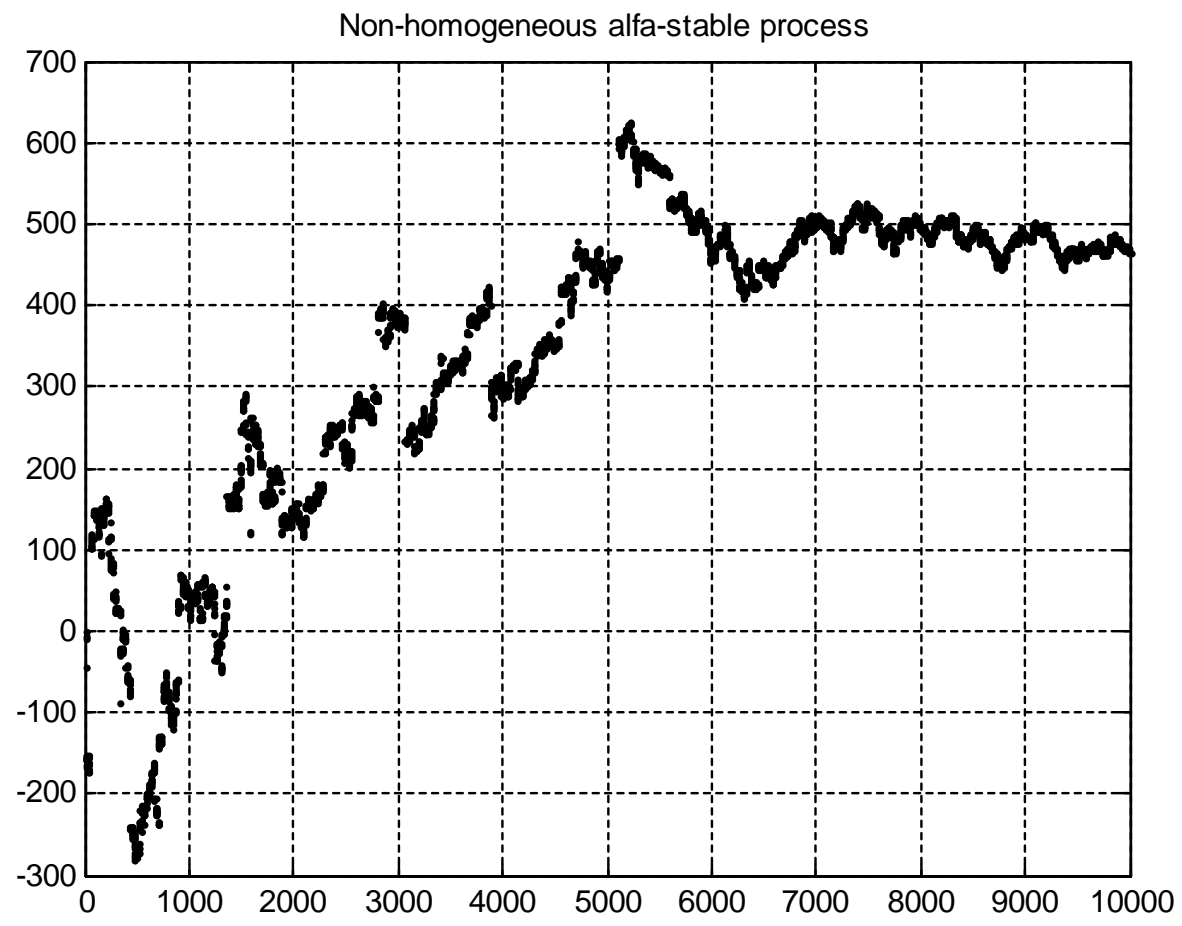

Figure(3): Non-homogeneous $\alpha$-stable process, where $\alpha$ goes from 1 to 2 (Brownian case)

Chambers, Mallows and Stuck (1976) describe a simulation method for generating $\alpha$-stable processes with a set of admissible parameters, and also provide a list of Fortran programs to simulate this process. Figure (3) shows us a sample path of a non-homogeneous $\alpha$-stable process where $\alpha$ moves uniformly with the time from 1 to 2 (Brownian case).

\subsubsection{The non-homogeneous Tempered Stable Process}

The class of the Tempered Stable (TS) distributions was proposed by Tweedie (1984) and Koponen (1995), but this class of distributions may be generalized to the so called class of Modified Stable distributions due to Barndorff-Nielsen and Shephard (2003) and Rosinski (2006). The distribution function is not available in closed form but the characteristic function of the Tempered Stable (TS) distribution law $T S(\lambda, a, b)$ with $a>0, b \geq 0$ and $0>\lambda>1$, is given by

$$
\hat{\mu}(z ; \lambda, a, b)=\exp \left(a b-a\left(b^{1 / \lambda}-2 i z\right)^{\lambda}\right)
$$

We can define the non-homogeneous Tempered Stable $(T S)$ process $G=\left\{G_{t}: t \geq 0\right\}$ as the process which starts at zero, has independent increments and is stochastically continuous with piecewise stationary increments. It has an infinitely divisible distribution and, from the characteristic function, we can derive the Lévy measure of the non-homogeneous TS process

$$
v_{j}(d g)=a_{j} 2^{\lambda} \frac{\lambda}{\Gamma(1-\lambda)} g^{-\lambda-1} \exp \left(-\frac{1}{2} b_{j}^{1 / \lambda} g\right) 1_{\{g>0\}} d g
$$

The process is a subordinator and has infinite activity. The first term of the characteristic triplet is 
given by

$$
\gamma_{j}=a_{j} 2^{\lambda} \frac{\lambda}{\Gamma(1-\lambda)} \int_{0}^{1} g^{-\lambda} \exp \left(-\frac{1}{2} b_{j}^{1 / \lambda} g\right) d g
$$

As we have mentioned in the case of the $\alpha$-stable case, neither the density function nor specific random number generators are available. In order to simulate other techniques are available in the literature. The most common method is based on the so-called rejection-method by Rosinski (2002).

\subsubsection{The non-homogeneous Variance Gamma Process}

The class of Variance Gamma $(V G)$ distributions was introduced by Madan and Seneta (1990) and Madan and Milne (1991) as a model for stock returns, and it generates a finite variation process with infinite but relatively low activity of small jumps. The $V G$ process proposed in Madan et al. (1998) is obtained by evaluating arithmetic Brownian motion with drift $\theta$ and volatility $\sigma$ at a random time given by a gamma process having a mean rate per unit time of 1 and a variance rate of $v$. Specifically, we have

$$
G_{t}^{V G}(\sigma, v, \theta)=\theta G_{t}^{v}+\sigma W_{G_{t}^{v}}
$$

where $G_{t}^{v}$ is the gamma process with mean rate 1 and variance rate $v$, independent of $W$.
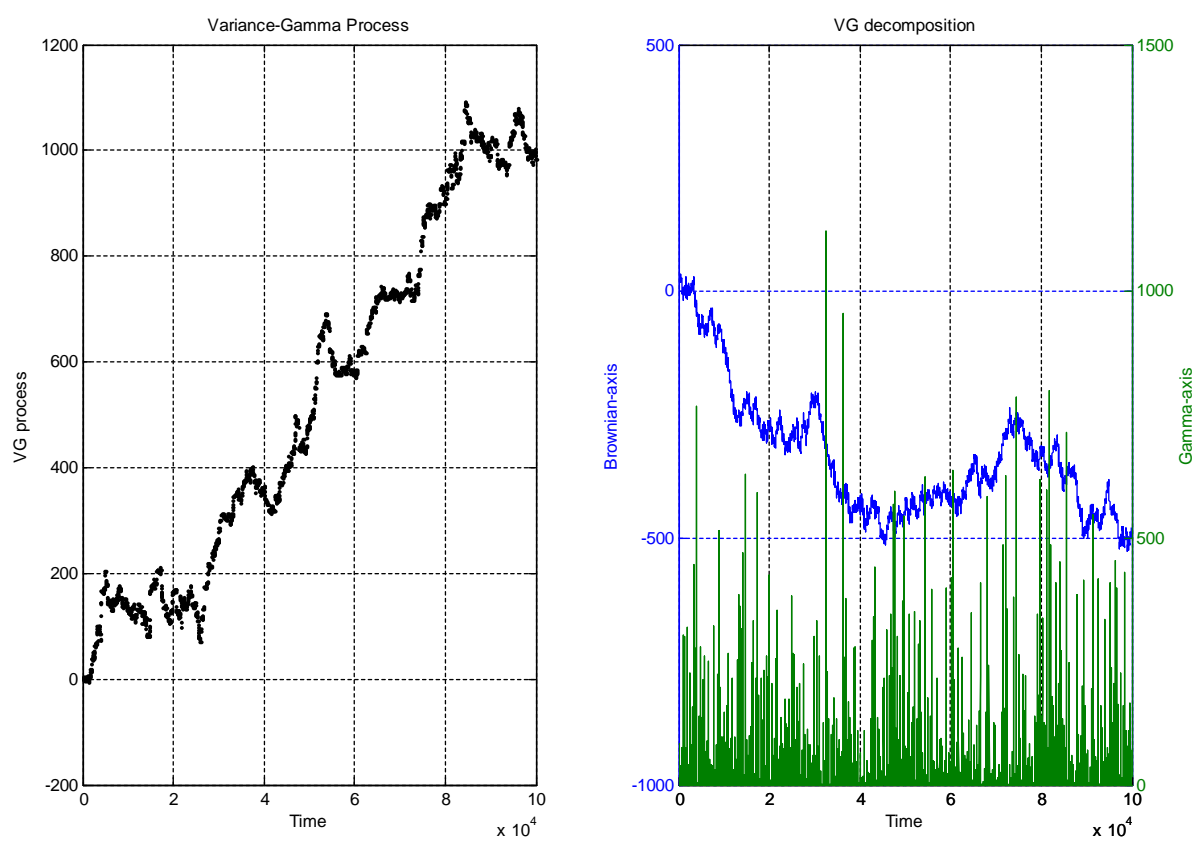

Figure (4): Simulation of a VG Process as a Time-Changed Brownian Motion

Figure (4) shows this composition of processes on the right side, and the resulting process $G_{t}(\sigma, v, \theta)$ (on the left side) is a pure jump process with infinite activity that has two additional parameters, providing control over skewness and kurtosis, respectively.

The characteristic function of the $V G(\sigma, v, \theta)$ law is easily evaluated as

$$
\hat{\mu}(z ; \sigma, v, \theta)=\left(1-i z \theta v+\frac{1}{2} \sigma^{2} v z^{2}\right)^{-1 / v}
$$


Hence, we can define the non-homogeneous Variance Gamma $(V G)$ process $\left\{G_{t}: t \geq 0\right\}$ as the process that starts at zero, has independent and piecewise stationary increments, with the following characteristic function

$$
\begin{aligned}
\mathbb{E}\left(\exp \left[i z G_{t}\right]\right) & =\prod_{j \leq \eta(t)} \mathbb{E}\left(\exp \left[i z\left(G_{t \wedge T_{j+1}}-G_{T_{j}}\right)\right]\right) \\
& =\prod_{j \leq \eta(t)}\left(1-i z \theta_{j} v_{j}+\frac{1}{2} \sigma_{j}^{2} v_{j} z^{2}\right)^{-\left(t \wedge T_{j+1}-T_{j}\right) / v_{j}}
\end{aligned}
$$

The Lévy density for the variance gamma process may be derived directly from the Lévy-Khintchine theorem. Alternatively, one may exploit the representation of the variance gamma process as the difference of two independent gamma processes. It is shown in Carr et al. (2002) that this characterization leads to the following Lévy measure

$$
v_{j}(d g)= \begin{cases}C_{j} \exp \left(G_{j} g\right)|g|^{-1} d g & g<0 \\ C_{j} \exp \left(G_{j} g\right)|g|^{-1} d g & g>0\end{cases}
$$

for any $j=0,1, \ldots, n$ where

$$
\begin{aligned}
C_{j} & =1 / v_{j}>0 \\
G_{j} & =\left(\sqrt{\frac{1}{4} \theta_{j}^{2} v_{j}^{2}+\frac{1}{2} \sigma_{j}^{2} v_{j}}-\frac{1}{2} \theta_{j} v_{j}\right)^{-1}>0 \\
M_{j} & =\left(\sqrt{\frac{1}{4} \theta_{j}^{2} v_{j}^{2}+\frac{1}{2} \sigma_{j}^{2} v_{j}}+\frac{1}{2} \theta_{j} v_{j}\right)^{-1}>0
\end{aligned}
$$

With this parametrization, we are implicitly assuming that the non-homogeneous VG process is expressed as the difference of two independent non-homogeneous Gamma processes, where $G^{(1)}$ is a Gamma processes with parameters $a_{j}=C_{j}$ and $b_{j}=M_{j}$, whereas $G^{(2)}$ is an independent Gamma process with $a_{j}^{\prime}=C_{j}^{\prime}$ and $b_{j}^{\prime}=G_{j}^{\prime}$. Figure (5) shows how these two mentioned gamma processes (right side) can generated the sample-path for the variance gamma process (left side).
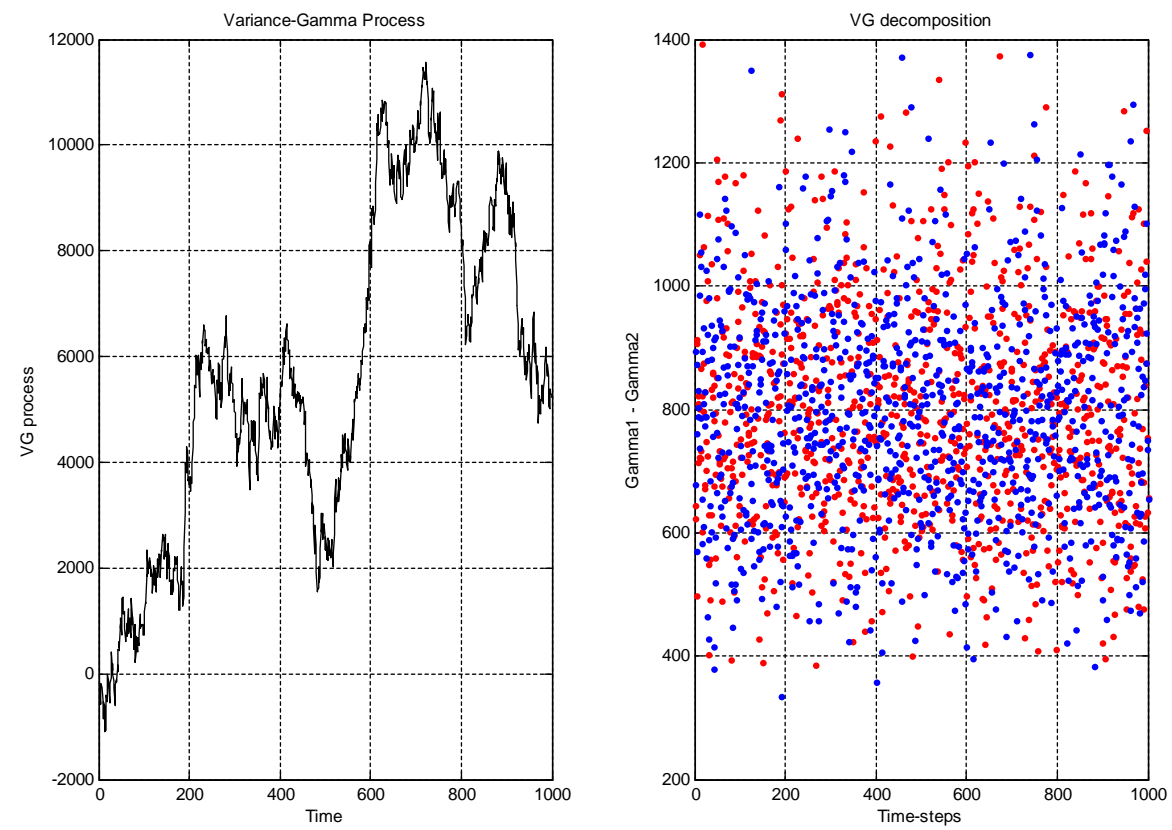
Figure (5): Simulation of a VG Process as the Difference of Two Gamma Processes

On the other hand, the Lévy measure has infinite mass, and hence a VG process has infinitely many jumps in any finite interval. Since

$$
\int_{-1}^{1}|g| v_{j}(d g)<\infty
$$

a VG process has paths of finite variation. A VG process has no Brownian motion component and its characteristic triplet is given by $\left[\gamma_{j}, 0, v_{j}(d g)\right]$ where

$$
\gamma_{j}=\frac{-C_{j}\left(G_{j}\left(\exp \left(-M_{j}\right)-1\right)-M_{j}\left(\exp \left(-G_{j}\right)-1\right)\right)}{M_{j} G_{j}}
$$

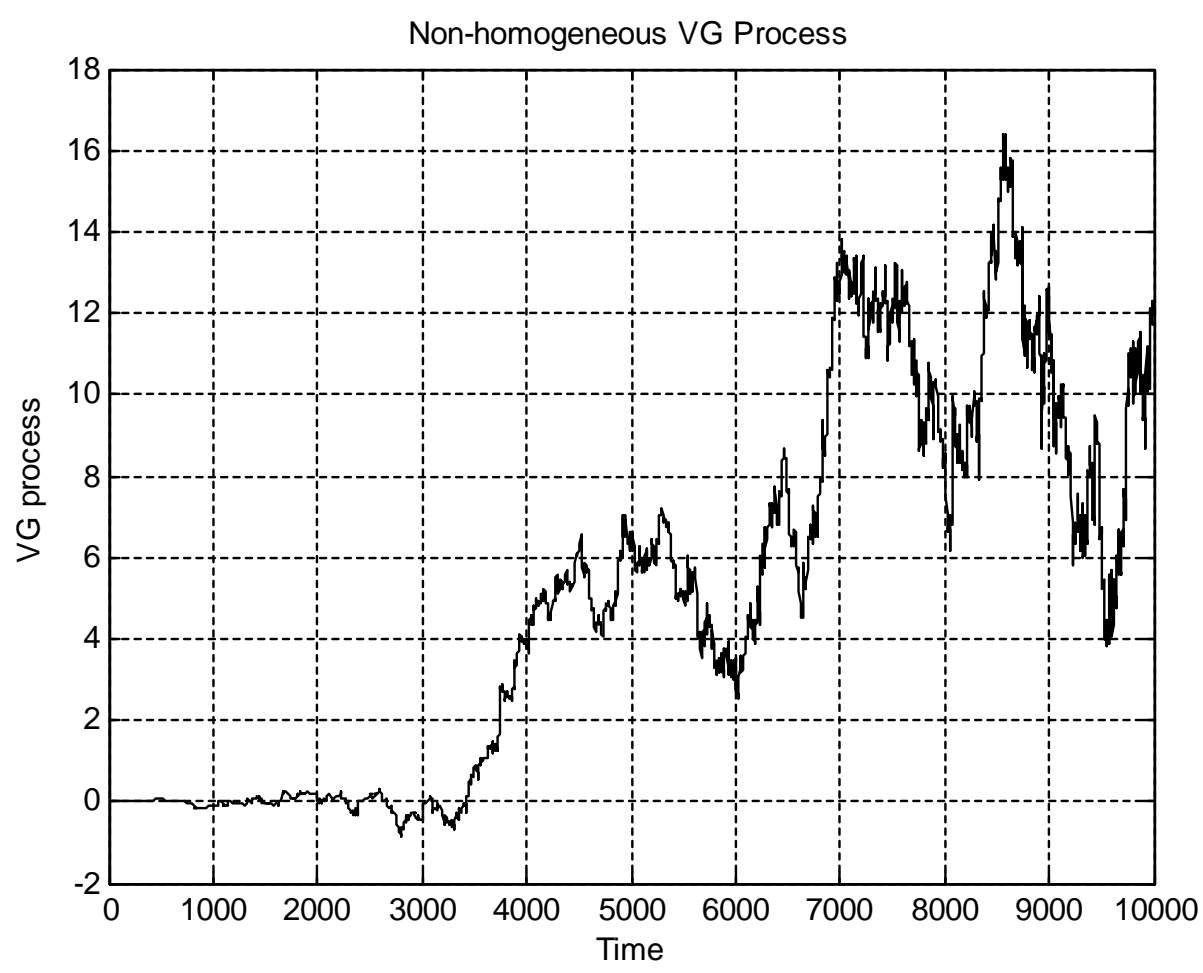

Figure (6): Non-homogeneous Variance Gamma Process.

\subsubsection{The non-homogeneous Normal Inverse Gaussian Process}

Following Barndorff-Nielsen (1995), the Normal Inverse Gaussian (NIG) distribution with parameters $\alpha>0,-\alpha<\beta<\alpha$ and $\delta>0, N I G(\alpha, \beta, \delta)$ has characteristic function

$$
\hat{\mu}(z ; \alpha, \beta, \delta)=\exp \left(-\delta\left(\sqrt{\alpha^{2}-(\beta+i z)^{2}}-\sqrt{\alpha^{2}-\beta^{2}}\right)\right)
$$

and we can define the non-homogeneous NIG process $\left\{G_{t}: t \geq 0\right\}$ as a process with $G_{\{0\}}=0$ a.s. with independent NIG distributed increments, continuous in probability and piecewise stationary. 
The Lévy measure for the NIG process is given by

$$
v_{j}(d g)=\frac{\delta_{j} \alpha_{j}}{\pi} \frac{\exp \left(\beta_{j} g\right) K_{1}\left(\alpha_{j}|g|\right)}{|g|} d g
$$

where $K_{\lambda}(g)$ denotes the modified Bessel function of the third kind with index $\lambda$.

A NIG process has no Brownian component and its Lévy triplet is given by $\left[0, \gamma_{j}, v_{j}(d g)\right]$ where

$$
\gamma_{j}=\frac{2 \delta_{j} \alpha_{j}}{\pi} \int_{0}^{1} \sinh \left(\beta_{j} g\right) K_{1}\left(\alpha_{j} g\right) d g
$$
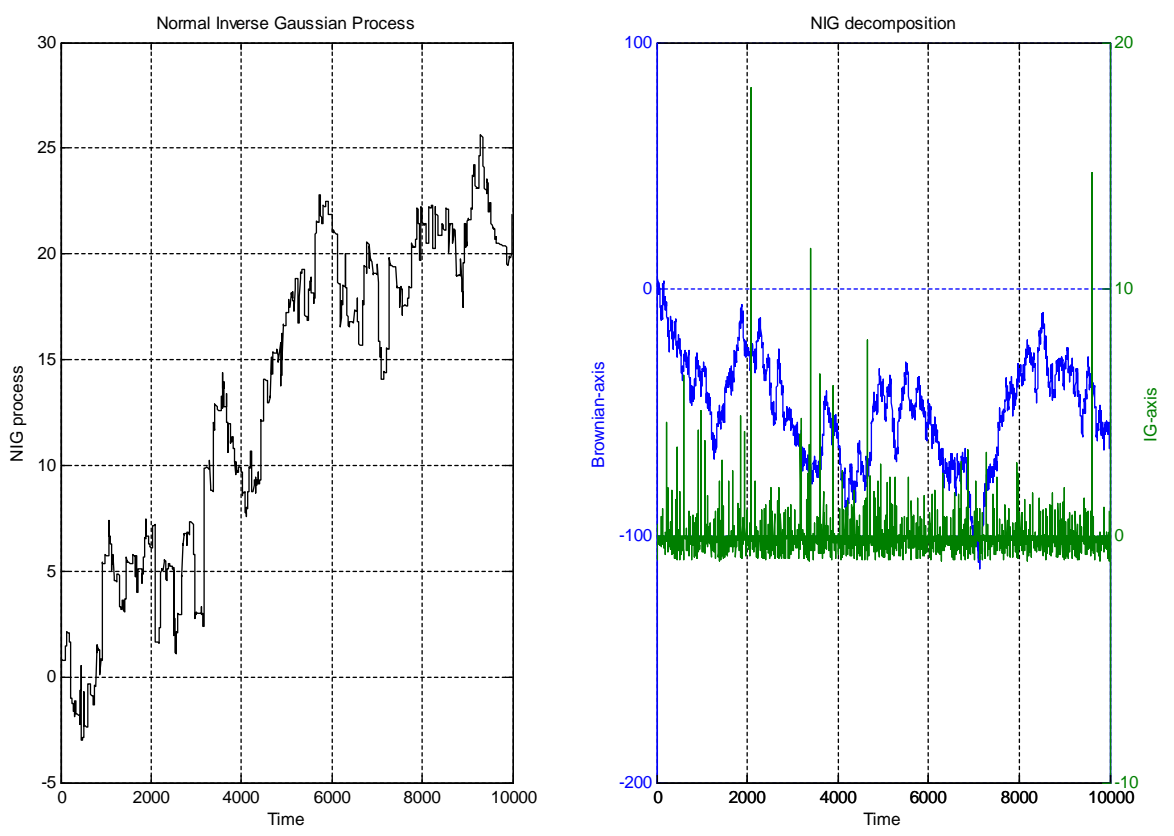

Figure (7): Simulation of a NIG process as a Time-Changed Brownian Motion

As in the VG case, we can also simulate an NIG process as a time-changed Brownian motion. Figure (7) shows a path of an NIG process (left side) obtained by sampling a standard Brownian motion and an IG process (right graph).

\subsubsection{The non-homogeneous CGMY Process}

In order to obtain a more flexible process than the Variance Gamma process, that has finite or infinite activity and infinite variation, the additional parameter $Y$ was introduced by Carr, Madan, Geman and Yor (2002). Later, in Carr et al. (2003), this four-parameter distribution was generalized to a six-parameter case, however we present here the first case, the $\operatorname{CGMY}(C, G, M, Y)$ distribution, with characteristic function

$$
\hat{\mu}(z ; C, G, M, Y)=\exp \left(C \Gamma(-Y)\left((M-i z)^{Y}-M^{Y}+(G+i z)^{Y}-G^{Y}\right)\right)
$$




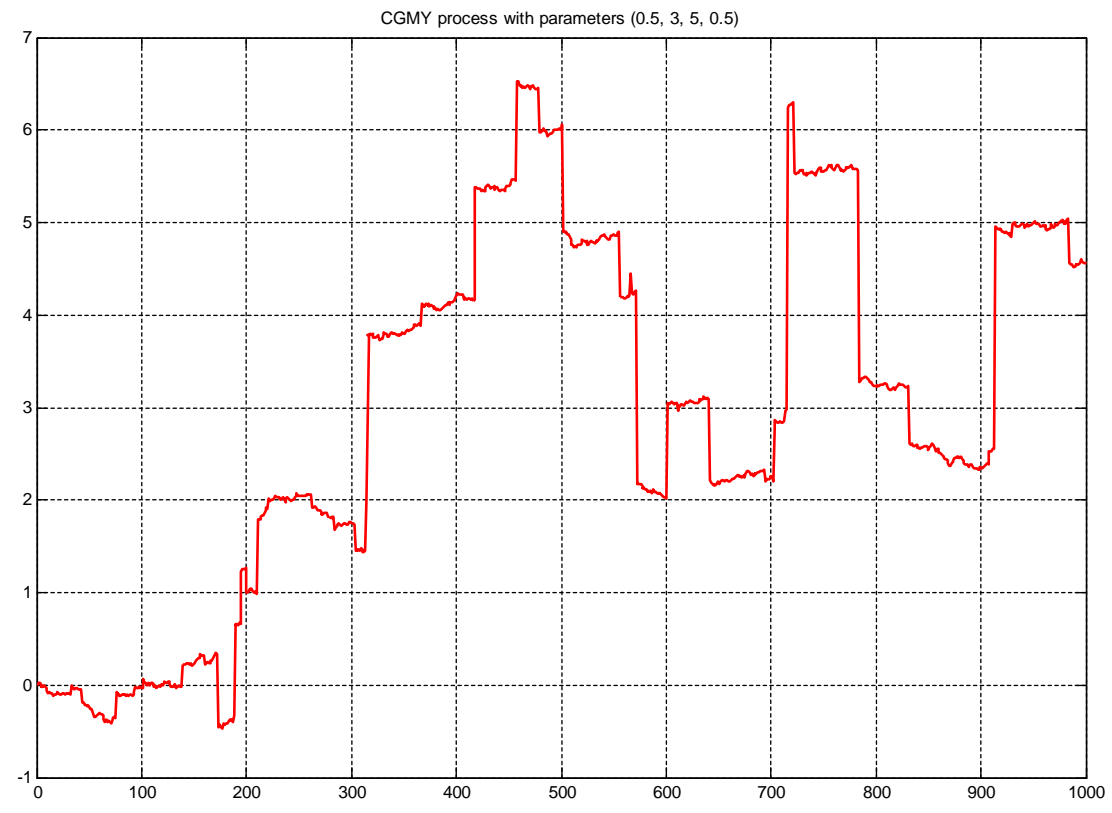

Figure (8): Simulation of a CGMY process

Based on this distribution, we can define a non-homogeneous CGMY process $\left\{G_{t}: t \geq 0\right\}$ as the process that starts at zero, has independent and piecewise stationary increments, with the following characteristic function

$$
\begin{aligned}
\mathbb{E}\left(\exp \left[i z G_{t}\right]\right) & =\prod_{j \leq \eta(t)} \mathbb{E}\left(\exp \left[i z\left(G_{t \wedge T_{j+1}}-G_{T_{j}}\right)\right]\right) \\
& =\prod_{j \leq \eta(t)} \hat{\mu}\left(k ; C_{j}, G_{j}, M_{j}, Y_{j}\right)^{\left(t \wedge T_{j+1}-T_{j}\right)} \\
& =\prod_{j \leq \eta(t)} \hat{\mu}\left(k ;\left(t \wedge T_{j+1}-T_{j}\right) C_{j}, G_{j}, M_{j}, Y_{j}\right) \\
& =\prod_{j \leq \eta(t)} \exp \left(C_{j}\left(t \wedge T_{j+1}-T_{j}\right) \Gamma\left(-Y_{j}\right)\left(\left(M_{j}-i z\right)^{Y_{j}}-M_{j}^{Y_{j}}+\left(G_{j}+i z\right)^{Y_{j}}-G_{j}^{Y_{j}}\right)\right)
\end{aligned}
$$

The CGMY process is a pure jump process with triplet $\left[\gamma_{j}, 0, v_{j}(d g)\right]$, that is, it contains no Brownian part. The path behavior is determined by the $Y_{j}$ parameter, which has a value restricted to $Y_{j}<2$ for every $j=0,1, \ldots, n$. If $Y_{j}<0$ the paths have finite jumps in any finite interval; if not, the paths have infinitely many jumps in any finite time interval, i.e. the process has infinite activity. Moreover, if the $Y_{j} \in[1,2)$, the process is of infinite variation.

The Lévy measure for the nonhomogeneous CGMY process is given by

$$
v_{j}(d g)= \begin{cases}C_{j} \exp \left(G_{j} g\right)|-g|^{-1-Y} d g & g<0 \\ C_{j} \exp \left(-G_{j} g\right)|g|^{-1-Y} d g & g>0\end{cases}
$$

and the first parameter of the characteristic triplet equals

$$
\gamma_{j}=C_{j}\left(\int_{0}^{1} \exp \left(-M_{j} g\right) g^{-Y_{j}} d g-\int_{-1}^{0} \exp \left(G_{j} g\right) g^{-Y_{j}} d g\right)
$$




\subsubsection{The non-homogeneous Meixner Process}

The Meixner process was introduced in Schoutens and Teugels (1998), Schoutens (2000) and Grigelionis (1999) later suggested that it may serve for fitting stock returns. This application to finance was worked out in Schoutens (2001, 2002). The density of the Meixner distribution $(\operatorname{Meixner}(\alpha, \beta, \delta))$ is given by

$$
\mu(g ; \alpha, \beta, \delta)=\frac{(2 \cos (\beta / 2))^{2 \delta}}{2 \alpha \pi \Gamma(2 d)} \exp \left(\frac{\beta g}{\alpha}\right)\left|\Gamma\left(\delta+\frac{i g}{\alpha}\right)\right|^{2}
$$

where $\alpha>0,-\pi<\beta<\pi, \delta>0$. The characteristic function of the Meixner $(\alpha, \beta, \delta)$ distribution is given by

$$
\hat{\mu}(z ; \alpha, \beta, \delta)=\left(\frac{\cos (\beta / 2)}{\cosh ((\alpha z-i \beta) / 2)}\right)^{2 \delta}
$$

Hence we can define the Meixner process $\left\{G_{t}: t \geq 0\right\}$ as the process that starts at zero, has independent and piecewise stationary increments, with a distribution given by the Meixner distribution function Meixner $\left(\alpha_{j}, \beta_{j}, \delta_{j} t\right)$.

According to Grigelionis (1999), this non-homogeneous Meixner process has no Brownian part while the pure jump part is governed by the Lévy measure

$$
v_{j}(d g)=\delta_{j} \frac{\exp \left(\beta_{j} g / \alpha_{j}\right)}{g \sinh \left(\pi g / \alpha_{j}\right)} d g
$$

The first parameter in the characteristic triplet equals

$$
\gamma_{j}=\alpha_{j} \delta_{j} \tan \left(\beta_{j} / 2\right)-2 \delta_{j} \int_{1}^{\infty} \frac{\sinh \left(\beta_{j} g / \alpha_{j}\right)}{\sinh \left(\pi g / \alpha_{j}\right)} d g
$$

This process has infinite variation due to $\int_{-1}^{+1}|g| v_{j}(d g)=\infty$, for any $j=0,1, \ldots, n$.

\subsubsection{The non-homogeneous Generalized Hyperbolic Process}

The Generalized Hyperbolic $(G H)$ distributions were introduced by Barndorff-Nielsen (1977) as a model for the grain-size distribution of wind-blown sand. In order to use this distribution in financial modelling, two subclasses of the $G H$ distribution appear in 1995. Eberlein and Keller (1995) used the Hyperbolic distribution and in the same year Barndorff-Nielsen (1995) proposed the NIG as a special case of a $G H$ distribution.

Following Barndorff-Nielsen (1977) the Generalized Hyperbolic $(G H)$ distribution $G H(\alpha, \beta, \delta, v)$ is defined through its characteristic function

$$
\hat{\mu}(k ; \alpha, \beta, \delta, v)=\left(\frac{\alpha^{2}-\beta^{2}}{\alpha^{2}-(\beta+i z)^{2}}\right)^{v / 2} \frac{K_{v}\left(\delta \sqrt{\alpha^{2}-(\beta+i z)^{2}}\right)}{K_{v}\left(\delta \sqrt{\alpha^{2}-\beta^{2}}\right)}
$$

where $K_{v}$ is the modified Bessel function, $\alpha$ and $\beta$ determine the shape of the distribution and $\delta$ is the scale of the parameter.

The density of the $G H(\alpha, \beta, \delta, v)$ distribution is given by

$$
\mu(g ; \alpha, \beta, \delta, v)=a(\alpha, \beta, \delta, v)\left(\delta^{2}+g^{2}\right)^{\frac{v}{2}-\frac{1}{4}} K_{v-\frac{1}{2}}\left(\alpha \sqrt{\delta^{2}+g^{2}}\right) \exp (\beta g)
$$


with

$$
a(\alpha, \beta, \delta, v)=\frac{\left(\alpha^{2}-\beta^{2}\right)^{v / 2}}{\sqrt{2 \pi} \alpha^{v-1 / 2} \delta^{v} K_{v}\left(\delta \sqrt{\alpha^{2}-\beta^{2}}\right)}
$$

where

$$
\begin{aligned}
& \delta \geq 0, \quad|\beta|<\alpha \quad \text { if } \quad v>0 \\
& \delta>0, \quad|\beta|<\alpha \quad \text { if } \quad v=0 \\
& \delta>0, \quad|\beta| \leq \alpha \quad \text { if } \quad v<0
\end{aligned}
$$

and using this distribution and characteristic function, we can define a non-homogeneous GH process $\left\{G_{t}: t \geq 0\right\}$ as the process that starts at zero, has independent and piecewise stationary increments, and where the distribution of $G_{t}$ has characteristic function

$$
\begin{aligned}
\mathbb{E}\left(\exp \left[i z G_{t}\right]\right) & =\prod_{j \leq \eta(t)} \mathbb{E}\left(\exp \left[i z\left(G_{t \wedge T_{j+1}}-G_{T_{j}}\right)\right]\right) \\
& =\prod_{j \leq \eta(t)} \hat{\mu}_{j}\left(g ; \alpha_{j}, \beta_{j}, \delta_{j}, v_{j}\right)^{\left(t \wedge T_{j+1}-T_{j+1}\right)}
\end{aligned}
$$

It is an infinite variation process without Gaussian part (in the general case). The Lévy measure $v_{j}(d g)$ is known, but the expression is rather complicate as it involves integrals of special functions. 
Chapter 2

Reaction-Additive systems to modelling Corporate Bonds: no-arbitrage and weak convergence conditions 


\subsection{Preliminaries}

\subsubsection{Introduction}

Notoriously, works in mathematical finance should reflect the market reality, and they have to be comprehensible for practitioners. Unfortunately, the ones which are realistic are not necessarily comprehensible and those comprehensible are not necessarily realistic.

But both are needed. Usually the trade-off between reality and simplicity in modelling is not easy to break, and unfortunately, the mathematics of finance are not effortless, and much market practice is based on soft or partial use of these tools, working and pricing with models that do not reflect in a complete manner what is actually going on.

Basically the main goal of this Second Chapter $^{1}$ is to develop a sufficiently wide model for corporate bonds with credit risk, and develop a set of mathematical tools and results that would allow the practitioner to simplify this framework and conditions in order to implement these models according to the specific needs of the market (with or without continuity and with or without jumps, with or without credit migration or under different types of default).

But additionally, we recognize that it is true that continuous-time models and pricing rules, presented in this chapter, are often too complex to handle. Therefore it is convenient to both discretize time and space and show that the discretization is good in the sense that the discretized models and pricing rules converge to the continuous-time model as the discretization steps tend to zero. An additional goal of this chapter is the treatment of this aspect of mathematical finance. We study both the approximation of a continuous-time model by a sequence of discrete-time models with credit risk, and the convergence of price processes under a framework that provides the practitioner a multiple set of models and credit conditions.

Therefore, in this Second Chapter, we present basically two results:

- First, a new framework to model interest rates with credit risk and a new approach to price corporate bonds with credit migration, under risk-neutral probability. We derive the non-arbitrage conditions under different conditions of recovery, and we obtain new expressions in order to estimate the probabilities of default under risk-neutral measure.

- Second, we investigate the conditions of weak convergence in incomplete markets, and more specifically, we study the weak convergence conditions of a composition of processes, between a semimartingale and an additional process that represent the untradeable risk factor (credit risk or credit migration). Obviously, these results have direct applications to our model.

This chapter is organized as follows:

- In Section 1 we introduce the basics such as definitions and technical notation that will be used during the whole chapter. Additionally, we expose here the different assumptions about the dynamics of forward rate models under a semimartingale framework.

\footnotetext{
${ }^{1}$ This work has been developed under the direction of Javier Nogales and Winfried Stute. I am extremely grateful to them. I also benefited greatly in my work from discussions with seminar participants at Statistical Lab. at University of Cambridge (Oct.2006), participants at IV Bachelier Congress in Tokyo (2006), and very specially with Rama Cont, David Nualart, C. Rogers, W. Runggaldier and T. Schmidt. Their advice, suggestions and assistance has been key in the development of this work. Comments and suggestions are welcome, all errors are my own.

Contact email: jpcolino@gmail.com
} 
- Section 2 is devoted to develop the basic expressions for corporate bonds under different recovery frameworks, basically extending the results of Heath, Jarrow and Morton (1992) to our framework.

- In Section 3 we obtain the no-arbitrage expressions for each model and we derive a risk-neutral form for the probability of default.

- Section 4 is mainly devoted to show some relevant results related with the reaction-additive system. Firstly, we expose very briefly different methodologies that guarantee the existence of a solution to our system. Second, assuming the existence of this solution, we show the Markov property and uniqueness, and finally we prove that this solution has sample-paths in the Skorohod space.

- Finally, Section 5 analyzes the conditions for weak convergence of this system in the Skorohod space, but not only from the theoretical point of view but also we obtain the conditions in terms of the sequence of triplets that characterize the distributions.

\subsubsection{Basic Assumptions for the risk-free Interest Rates model.}

We will consider processes on a complete stochastic basis $(\Omega, \mathcal{G}, \mathbb{P})$. Let $G=\left\{G_{t} ; t \geq 0\right\}$ be the LIBOR additive process with a given tenor structure $0=T_{0}<T_{1}<\ldots<T_{n}=T^{\star}$ with $T^{\star}$ fixed, according to the definitions given in the previous chapter.

Now, the LIBOR additive process $G$ is introduced here as a source of uncertainty in our model. Notice that the trajectories of this process belong to the Skorohod space $\mathcal{D}$. We can associate with $G_{t}$ a random measure of its jumps, denoted by $\mu_{\eta(t)}$ for any $t \in\left[0, T^{\star}\right], A \in \mathcal{B}\left(\mathbb{R}^{d}\right)$ and $t \in\left[T_{\eta(t)}, T_{\eta(t)+1}\right)$. Actually, set

$$
\mu_{\eta(t)}([0, t], A)=\sum_{0<s \leq t} 1_{A}(\triangle G(s))
$$

and let us introduce the measure $v_{\eta(t)}$ as

$$
v_{\eta(t)}(A)=\mathbb{E}\left(\mu_{\eta(t)}([0, t], A)\right)
$$

is called the Lévy measure of the process $G$.

The Lévy-Khintchine formula (Theorem 31) and Theorem 34 in the previous Chapter, have shown that the characteristic function has the form:

$$
\begin{aligned}
\hat{\mu}_{t}(z) & =\mathbb{E}(\exp [i\langle z, G(t)\rangle]) \\
& =\prod_{j \leq \eta(t)} \mathbb{E}\left(\exp \left[i\left\langle z,\left(G_{t \wedge T_{j+1}}-G_{T_{j}}\right)\right\rangle\right]\right) \\
& =\exp \left[\sum_{j \leq \eta(t)}\left(t \wedge T_{j+1}-T_{j}\right) \psi_{j}(z)\right]
\end{aligned}
$$

with $z \in \mathbb{R}^{d}$ and

$$
\psi_{j}(z)=i\left\langle\gamma_{j}, z\right\rangle-\frac{1}{2}\left\langle z, A_{j} z\right\rangle+\int_{\mathbb{R}^{d}}\left(e^{i\langle z, g\rangle}-1-i\langle z, g\rangle 1_{\{|g| \leq 1\}}\right) v_{j}(d g), \quad j=0,1, \ldots, n
$$

and where $A_{j}$ is a symmetric nonnegative-definite $d \times d$ matrix, $\gamma_{j} \in \mathbb{R}^{d}, v_{j}$ is the mentioned Lévy measure on $\mathbb{R}^{d} \backslash\{0\}$ and $g \in \mathbb{R}^{d}$ satisfying

$$
v_{j}(\{0\})=0 \quad \text { and } \quad \int_{\mathbb{R}^{d}}\left(|g|^{2} \wedge 1\right) v_{j}(d g)<\infty
$$

for any $j=1, \ldots, n$. 
Under these two conditions, and using Jacod and Shiryaev (2003, II.2.29), $G$ is a special semimartingale and $G$ has a well known Lévy-Itô decomposition or canonical representation (assuming jumps bounded by a truncation constant):

$$
\begin{aligned}
G(t)= & G(0)+\sum_{j \leq \eta(t)} \int_{T_{j}}^{t \wedge T_{j+1}} \alpha_{j}(s) d s+\sum_{j \leq \eta(t)} \int_{T_{j}}^{t \wedge T_{j+1}} \sigma_{j}(s) W(d s) \\
& +\sum_{j \leq \eta(t)} \int_{T_{j}}^{t \wedge T_{j+1}} \int_{|x| \leq 1} x\left(v_{j}-\mu_{j}\right)(d s, d x)
\end{aligned}
$$

where $\alpha_{\eta(s)} \in \mathbb{R}^{d}, W$ is a standard d-dimensional Wiener process with values in $\mathbb{R}^{d}$ and $\sigma_{\eta(t)}$ is a measurable version of the square-root of the diagonal elements of a symmetric nonnegative-definite d-dimensional matrix $A_{\eta(t)}=\left(\sigma_{\eta(t)}(i, j)\right)_{i, j \leq d}$.

Let $r(t), t \geq 0$ be the short rate process. If at moment 0 one puts into the bank account 1 unit, then at moment $t$ one has

$$
B_{t}=\exp \left[\int_{0}^{t} r(s) d s\right]
$$

Let $B(t, T)$ be the market price at moment $t$ of a bond paying 1 unit at maturity time $T$. The forward rate $f(t, T)$ curve is a function defined for $t \leq T<T^{\star}$ and such that

$$
B\left(t, T_{i}\right)=\exp \left[-\int_{t}^{T_{i}} f(t, s) d s\right]
$$

We postulate here the following dynamic for the forward rates

$$
d f(t, T)=\alpha_{\eta(t)}(t, T) d t+\sigma_{\eta(t)}(t, T) d W_{t}+\int_{E} h(t, T, x) \mathbf{1}_{\{|x| \leq 1\}}\left(\mu-v_{\eta(t)}\right)(d t, d x)
$$

Notice that the usual short rate is defined as $r(t)=f(t, t)$.

\subsubsection{Basic Assumptions for the Credit Risk Model}

In this section, we mainly focus on corporate (defaultable) bond featuring two different issues,

- first, the dynamic of defaultable instantaneous forward rates in incomplete markets, which are specified through the Heath, Jarrow and Morton (1992) model, driven by a LIBOR additive processes,

- and second, we additionally assume that the credit migration is modelled by a SDE driven by a multivariate marked point process.

In order to achieve this aim, we have to establish some assumptions that will be applied during the whole work.

\section{Assumptions related with the Credit Risk dynamic}

1. Given a fixed horizon date $T^{\star} \in \mathbb{R}_{+}$, let us assume that our continuous-time financial economy 'lives' on a "sufficiently rich" stochastic basis $(\Omega, \mathbb{F}, \mathbb{P})$ endowed with the filtration $\mathbb{F}=\left(\mathcal{F}_{t}\right)_{t \in\left[0, T^{\star}\right]}$. 
Notice that in our case, a "sufficiently rich" stochastic basis is one such that the filtration is generated by two stochastic processes $G$ and $C$

$$
\mathcal{F}_{t}=\sigma\left\{G_{s}, C_{s} ; 0 \leq s \leq t\right\}
$$

which satisfies the "usual conditions". Notice that we can define the embedded filtration $\mathcal{G}_{t} \subseteq \mathcal{F}_{t}$ such that

$$
\mathcal{G}_{t}=\sigma\left\{W_{s}, v([0, s] \times E) ; 0 \leq s \leq t, E \in \mathcal{B}\left(\mathbb{R}^{d}\right)\right\}
$$

and additionally, we can define as well a second embedded filtration $\mathcal{C}_{t} \subseteq \mathcal{F}_{t}$ such that

$$
\mathcal{C}_{t}=\sigma\left\{C_{s} ; 0 \leq s \leq t\right\}
$$

Henceforth, we can define $\mathcal{F}_{t}$ as the original full filtration such that

$$
\mathcal{F}_{t}=\mathcal{G}_{t} \vee \mathcal{C}_{t}=\sigma\left\{\mathcal{G}_{s}, \mathcal{C}_{s} ; 0 \leq s \leq t\right\}
$$

with respect to which all processes are adapted. In following subsections, assumptions about the nature of $G$ and $C$ are detailed.

Additionally to these three filtrations, we also have three smaller filtrations $\mathbb{F}^{\prime}, \mathbb{G}^{\prime}$ and $\mathbb{C}^{\prime}$ that will be called observed filtrations such that $\mathcal{F}_{t}^{\prime}=\mathcal{G}_{t}^{\prime} \vee \mathcal{C}_{t}^{\prime}$ and $\mathcal{F}_{t}^{\prime} \subseteq \mathcal{F}_{t}$. They are originated directly from the observed time series of market prices and notice that implicitly, we are assuming different notions of equivalent martingale measures, according to which filtration we are interested in.

2. We assume that the process $G$ is a LIBOR additive process. According to the results obtained in Chapter I, this process has an infinitely divisible and self-decomposable distribution and it admits the Lévy-Khintchine formula and the Lévy-Itô decomposition.

3. On the other hand, we are assuming also that the credit quality of corporate debt is represented by the random variable $C$ categorized into a finite number of (mutually disjoint) credit rating classes (credit classes, for short). Each credit class is represented by one of $m+1 \in \mathbb{N}^{+}$elements of a finite state space, say $\mathcal{K}=\left\{0, \frac{1}{m}, \frac{2}{m}, \ldots, \frac{m-1}{m}, 1\right\}$ (state space). By convention, the state 1 is always assumed to correspond to the default event. In addition, the states are ordered so that the state 0 represents the highest ranking, whereas the state $\frac{m-1}{m}$ represents the lowest ranking.

Let us define the credit migration process by $C_{t}$ for any $0 \leq t \leq T^{\star}$ as a random variable on $(\Omega, \mathcal{C}, \mathbb{P})$ adapted to the filtration $\mathcal{C}=\left(\mathcal{C}_{t}\right)_{t \in\left[0, T^{\star}\right]}$. Let us assume that the dynamic of this process can be defined by the following stochastic differential equation with values in $\left[0, T^{\star}\right] \times \mathcal{K}$

$$
d C_{t}=\sum_{a, b \in \mathcal{K}}(b-a) 1_{\left\{C_{t-}=a\right\}} d N_{a b}(t), \quad C_{0} \in \mathcal{K} \backslash\{1\}
$$

where both the $m+1$-vector point process $N_{t}=\left(N_{a, 0}(t), \ldots, N_{a, \frac{m-1}{m}}(t), N_{a, 1}(t)\right)$, such that

$$
N_{a b}(t) \text { has }(\mathbb{P}, \mathcal{C}) \text {-intensity } \lambda_{a b}(t) \text { for } a, b \in \mathcal{K}
$$

where $\lambda_{a b}:\left[0, T^{\star}\right] \times \mathbb{R}^{d} \rightarrow[0, \infty]$ are bounded functions with bounded gradients.

4. The double sequence $\left(\tau_{k}, C_{\tau_{k}}\right)_{k \geq 1}$ is called a $\mathcal{C}$-adapted multivariate marked point process ${ }^{2}$. Notice that the $\tau_{k}$ 's form a sequence of stopping times that define the moments of time that the credit rate $C$ changes. More explicitly, for any $k \in \mathbb{N}_{+}$the random variable (random stopping time) $\tau_{k}$ will be defined as

$$
\left\{\begin{array}{l}
\tau_{0}:=0 \\
\tau_{k}:=\inf \left\{t>\tau_{k-1} / C_{t} \neq C_{\tau_{k-1}}\right\} \wedge T^{\star} \\
\tau_{k}^{\star}:=\inf \left\{t>\tau_{k-1} / C_{t}=1\right\} \wedge T^{\star}
\end{array}\right.
$$

\footnotetext{
${ }^{2}$ See Liptser and Shiryaev (1989) 3.4 p.168 or Brémaud (1981) 2.1 p.19
} 
and it represents the time of the $k^{t h}$ jump or transition for $C$. Therefore, $\tau_{k}: \Omega \rightarrow \mathbb{R}_{+}$is a nonnegative random variable defined in the probability space $(\Omega, \mathcal{F}, \mathbb{P})$. For convenience, we assume for any $k \in \mathbb{N}_{+}$that $\mathbb{P}\left\{\tau_{k}=0\right\}=0$ and hence $\mathbb{P}\left\{\tau_{k}>0\right\}=1$.

Notice that the default time $\tau_{k}^{\star}$ is the first moment when the rating process hits the state 1 or reaches the state of default. Default is, by definition, an absorbing state and sometimes, and for the sake of simplicity, it will appear as $\tau^{\star}$.

5. The usual approach to continuous-time Markov chains is based on transition semigroups, and the principal mathematical object is then the infinitesimal generator. The transition semigroup is the continuous-time analogue of the iterates of the transition matrix in discrete time.

Given an initial rating $C_{0}$ of a defaultable bond, as in the discrete case, the future changes in its ratings are described by a $\mathcal{K}$ valued stochastic process $C_{t}$ referred to as the migration process under the real-world probability $\mathbb{P}$ that follows a continuous-time homogeneous $\mathbb{C}$-Markov chain, with the transition semigroup $P$ of the following form:

$$
\mathbb{P}(t)=\left[p_{a b}(t)\right]_{a, b \in \mathcal{K}}, \text { with } 0<t \leq T^{\star}
$$

where

$$
p_{a b}(t):=\mathbb{P}\left(C_{t+s}=b \mid C_{s}=a\right) \text { for every } s, t \in\left[0, T^{\star}\right]
$$

In a credit risk framework, we shall postulate that the default state $C(t)=1$ is absorbing, i.e. $p_{1,1}(t)=1$ or equivalently $p_{1, b}(t)=0$ for any $b \in \mathcal{K} \backslash\{1\}$.

On the other hand, it is also very well-known that the right-hand side continuity at time $t=0$ of $P(\cdot)$ implies the right-hand side differentiability at $t=0$. More specifically, the following finite limit exists for every $a, b \in \mathcal{K}$ and equals

$$
\lambda_{a b}:=\lim _{t \downarrow 0} \frac{p_{a b}(t)-p_{a b}(0)}{t}=\lim _{t \downarrow 0} \frac{p_{a b}(t)-\delta_{a b}}{t} .
$$

Observe that for every $a \neq b$ we have $\lambda_{a b} \geq 0$, and $\lambda_{a a}=-\sum_{a=0, a \neq b}^{1} \lambda_{a b}$. The matrix $\Lambda:=$ $\left[\lambda_{a b}\right]_{0 \leq a, b \leq 1}$ is called the infinitesimal generator matrix for a Markov chain associated with $P(\cdot)$. Since each entry of $\lambda_{a b}$ of the matrix $\Lambda$ can be shown to represent the intensity of transition from the state $a$ to the state $b$, the infinitesimal generator matrix $\Lambda$ is also commonly known as the intensity matrix.

\section{Assumptions related with the stochastic process}

1. Let us define the LIBOR additive process with the credit rating $G_{t}^{C}$ as a $\mathcal{F}_{t}$-adapted LIBOR additive process that is also a function of the credit state such that

$$
G_{t}^{C}=G(t, C(t))
$$

Notice that $\left(G_{t}\right)_{t \geq 0}$ is a $\mathcal{G}_{t^{-}}$adapted LIBOR additive process ${ }^{3}$ on $\mathbb{R}^{d}$, and $\left(C_{t}\right)_{t \geq 0}$ is a $\mathcal{C}_{t^{-}}$ adapted multivariate point process on $\mathcal{K}$. We have in mind a map from $\mathcal{D}^{d} \times \mathcal{D}$ into $\mathcal{D}^{d}$, where $\mathcal{D}^{d}=\mathcal{D}\left(\mathbb{R}^{d}, I\right)$, with $I=\left[0, T^{\star}\right] \subset \mathbb{R}^{+}$, is a d-dimensional Skorohod space and $\mathcal{D}=\mathcal{D}(\mathcal{K}, I)$ is also a Skorohod space, with $\mathcal{K}=\left\{0, \frac{1}{m}, \frac{2}{m}, \ldots, \frac{m-1}{m}, 1\right\}$ as a finite space in $[0,1]$, and $t \in\left[0, T^{\star}\right]$. For the sake of clarity, in the future the final d-dimensional Skorohod space will be denoted as $D_{\left[0, T^{\star}\right]}\left(\mathbb{R}^{d}, \mathcal{K}\right)$. Several proofs and developments in this context will be provided in sections $(2.4)$ and (2.5) in this chapter.

2. We will take for granted the structure of infinitely divisible distributions on $\mathbb{R}^{d}$, and in particular the Lévy-Khintchine formula. We have seen that $\left(G_{t}^{c}\right)_{t \geq 0}$ is a LIBOR additive process

\footnotetext{
${ }^{3}$ See Section 1.2. in Chapter 1 for the definitions and properties of LIBOR additive processes
} 
on $\mathbb{R}^{d}$, and for every $c \in \mathcal{K} \backslash\{1\}$ given, and for every $t, G_{t}^{c}$ has an infinitely divisible distribution, and the driving process $G_{t}^{c}$ has a triplet characteristic $\left(\gamma_{j}^{c}(t), A_{j}^{c}, \nu_{j}^{c}\right)_{j>0}$, that is connected with the mentioned canonical (Lévy-Itô) decomposition of $G^{c}$ for any $c \in \mathcal{K} \backslash\{1\}$ in the following manner:

$$
\begin{aligned}
G(t, c)= & G_{t}^{c} \\
= & G_{0}^{c}+\sum_{j \leq \eta(t)} \int_{T_{j}}^{t \wedge T_{j+1}} \alpha_{j}(u, c) d u+\sum_{j \leq \eta(t)} \int_{T_{j}}^{t \wedge T_{j+1}} \sigma_{j}(u, c) d W_{u} \\
& +\sum_{j \leq \eta(t)} \int_{T_{j}}^{t \wedge T_{j+1}} \int_{\mathbb{R}^{d}} \delta(u, x)\left(\mu_{j}^{c}-v_{j}^{c}\right)(d u, d x)
\end{aligned}
$$

where $\sigma_{\eta(t)}(u, c) d W_{t}$ is the continuous martingale part of $G_{t}^{c}$ and, on the other hand, $v_{\eta(t)}^{c}$ is the random measure associated with the jumps of $G_{t}^{c}$, and $\mu_{\eta(t)}^{c}(d u, d x)=d u F_{\eta(t)}^{c}(d x)$ is its (non-random) compensator.

Notice that $W_{t}$ is the usual standard $d$-dimensional Brownian motion and $\sigma_{\eta(t)}(u, c)$ is a $d$ dimensional vector that is the diagonal of the square-root of the symmetric nonnegative-definite matrix $A_{\eta(t)}^{c}$;

Also we had assumed that the process $G^{c}$ has jumps bounded by a constant $h=1$, however this truncation function can be any $h \in \mathbb{R}^{+}$by replacing $G^{c}$ by $G^{c} / h$ (which has jumps bounded by $1)$ and $\gamma_{\eta(t)}^{c}(t)$ by $h \gamma_{\eta(t)}^{c}(t)$, so the $\left(\mathcal{G}_{t}\right)^{c}$ and the rates are unchanged. It is clear that any martingale solution will depend on the choice of the truncation function. In the sequel we fix one truncation function and sometimes do not mention the dependence of the characteristics on this truncation function.

\section{Assumptions related with the forward rates with credit risk}

1. Let us define $f(t, T, c)$ as the instantaneous defaultable forward rates at time $t \in[0, T]$ for any $T<T^{\star}$ and for every $c \in \mathcal{K} \backslash\{1\}$. It corresponds to the rate that one can contract for a time $t$, on a loan with credit risk $c$ that begins at date $T$ and is returned an instant later. It is usually defined by

$$
f(t, T, c)=-\frac{\partial \log B(t, T, c)}{\partial T}
$$

where $B(t, T, c)$ is the value in $t$ of a zero-coupon bond conditional to the credit rate $c$ until maturity $T$, or in other words

$$
B(t, T, c):=\left.B\left(t, T, C_{t}\right)\right|_{C_{t}=c} \text { for every } c \in \mathcal{K} \backslash\{1\}
$$

and therefore, the conditional zero-coupon bond with maturity $T$ and the credit rate $c$ follows

$$
B(t, T, c)=\exp \left\{-\int_{t}^{T} f(t, s, c) d s\right\}
$$

2. We assume that the evolution of this forward rate is driven by a $d$-dimensional LIBOR additive process for a given credit rate $c \in \mathcal{K} \backslash\{1\}$ that admits the Lévy-Itô decomposition, such that the dynamics of the instantaneous forward rate $f(t, T, c)$ given the credit rating $c \in \mathcal{K} \backslash\{1\}$ in 
$t \leq T \in I$, under the real-world probability $\mathbb{P}$, which we assume as follows ${ }^{4}$ :

$$
d f(t, T, c)=\alpha_{\eta(t)}(t, T, c) d t+\sigma_{\eta(t)}(t, T, c) d W_{t}+\int_{E} \delta(t, T, x)\left(\mu_{\eta(t)}^{c}-v_{\eta(t)}^{c}\right)(d t, d x)
$$

when $t \leq T \in I$ where $\eta(t)=\sup \left\{j \geq 0: T_{j} \leq t\right\}$ with $j=0,1, \ldots, n$ (for the sake of clarity, we will denote this by the generic index $j$ ), $W_{t}$ is a $d$-dimensional standard Wiener process in $\mathbb{R}^{d}$ and it is identical for any $c \in \mathcal{K} \backslash\{1\}, \mu_{\eta(t)}^{c}$ is a random measure for a given credit rating $c \in \mathcal{K} \backslash\{1\}$ such that $\mu \in \mathbb{N}^{+}$with the compensator $v_{\eta(t)}^{c}(d t, d x)$.

Notice that a forward rate is not a financial asset issued by a company that has the probabilities of default, therefore $c:=C_{t} \mid \mathcal{F}_{t} \in \mathcal{K} \backslash\{1\}$ for every $t \in[0, T], T \in I$. This means that modelling these forward rates, we are not considering the probabilities of credit migration that usually appear in a specific corporate bond valuation.

On the other hand, we are implicitly assuming that $(f(t, T, c))_{c \in \mathcal{K} \backslash\{1\}}$ is a sequence of semimartingales, because $G^{c}$ is a semimartingale, and also the risk-free forward rate $f(t, T, 0):=f(t, T)$ is another semimartingale.

3. Let us make some assumptions on the coefficients. Basically the functions $\alpha_{j}: \Omega \times\left[0, T^{\star}\right] \times\left[0, T^{\star}\right] \rightarrow$ $\mathbb{R}$ and $\sigma_{j}: \Omega \times\left[0, T^{\star}\right] \times\left[0, T^{\star}\right] \rightarrow \mathbb{R}_{+}$for any $j=0,1, \ldots, n$ and both are $\mathcal{R}_{+} * \mathcal{B}\left(\left[0, T^{\star}\right]\right)$-measurable. The coefficient $\delta: \Omega \times \mathbb{R}^{r} \times\left[0, T^{\star}\right] \times\left[0, T^{\star}\right]$ is $\mathcal{R}_{+} * \mathcal{B}\left(\left[\mathbb{R}^{r}\right]\right) * \mathcal{B}\left(\left[0, T^{\star}\right]\right)$ measurable as well, and all the coefficients cited previously are finite for all times $t$, and fixed $T \geq t$, or in other words

$$
\begin{aligned}
& \sum_{j \leq \eta(t)} \int_{T_{j}}^{t \wedge T_{j+1}}\left(\sum_{\eta(u) \leq j \leq \eta(T)} \int_{t \vee T_{j}}^{T_{j+1} \wedge T}\left|\alpha_{j}(u, s, c)\right| d s\right) d u<\infty \\
& \sum_{j \leq \eta(t)} \int_{T_{j}}^{t \wedge T_{j+1}}\left(\sum_{\eta(u) \leq j \leq \eta(T)} \int_{t \vee T_{j}}^{T_{j+1} \wedge T}\left|\sigma_{j}(u, s, c)\right|^{2} d s\right) d u<\infty
\end{aligned}
$$

and

$$
\sum_{j \leq \eta(t)} \int_{T_{j}}^{t \wedge T_{j+1}} \int_{E}\left(\sum_{\eta(u) \leq j \leq \eta(T)} \int_{t \vee T_{j}}^{T_{j+1} \wedge T}|\delta(u, s, x)|^{2} d s\right) v_{j}^{c}(d u, d x)<\infty
$$

Notice that all coefficients are equal to zero for $T<t$, and we are assuming that $E=\mathbb{R}^{d}$. Also to abbreviate the formulae we will use $\tilde{\mu}_{j}^{c}:=v_{j}^{c}-\mu_{j}^{c}$ where $c \in \mathcal{K} \backslash\{1\}$, at this moment, is a fixed credit rate.

4. Additionally, for every $s, t, T \in\left[0, T^{\star}\right]$ and $s, t \leq T$, and $c \in \mathcal{K} \backslash\{1\}$ such that there is a constant $\tilde{C}<\infty$ and

$$
\begin{aligned}
\left|\alpha_{j}(s, T, c)-\alpha_{j}(t, T, c)\right| & \leq \tilde{C}|s-t| \\
\left|\sigma_{j}(s, T, c)-\sigma_{j}(t, T, c)\right| & \leq \tilde{C}|s-t| \\
|\delta(u, s, x)-\delta(u, t, x)| & \leq \tilde{C}|s-t|
\end{aligned}
$$

then the Equation (2.10) will admit a unique (strong) solution (see Fujiwara and Kunita (1989), Tang and Li (1994) or Protter (2004) Theorem V.6).

5. By definition $r_{t}^{c}=f(t, t, c)$ is the instantaneous spot rate or simply the spot rate given a credit rating (called in the literature also as short-rate). Also let us define the concept of instantaneous spread rate, as

$$
s\left(t, T_{j}, c\right):=f\left(t, T_{j}, c\right)-f\left(t, T_{j}, 0\right)
$$

\footnotetext{
${ }^{4}$ Notice that if $c=0$ we are considering the risk-free or default-free case.
} 


\section{Assumptions related with the Corporate Bond dynamic}

1. We consider a continuous-time trade economy for every $t$ inside the trading interval $\left[0, T^{\star}\right]$ with a fixed $T^{\star}>0$. Assume the existence of a (frictionless) continuous-time bond market where a set of assets $B\left(t, T, C_{t-}\right)$ stand for the price at time $t \leq T \leq T^{\star}$ of a zero-coupon bond with rating $C_{t-} \in \mathcal{K} \backslash\{1\}$, maturity at time $T \leq T^{\star}$ and recovery-rate $q$ in case of default.

2. For the sake of simplicity, let us select a subset of $n$-corporate bonds with maturity $T_{i}$ with $i=$ $0,1, \ldots, n$ and $T_{i} \leq T^{\star}$ for every $i=0,1, \ldots, n$. Notice that a (frictionless) market for $T_{i}$-corporate bonds with rating $C_{t-} \in \mathcal{K} \backslash\{1\}$ generate a family of bond prices for $i=0,1, \ldots, n$ with the same rating $C_{t-}$. It basically means a finite family of strictly positive real-valued adapted processes $B\left(t, T_{i}, C_{t-}\right)$, with $t \in\left[0, T_{i}\right]$, and the terminal (par) value at maturity $B\left(T_{i}, T_{i}, C_{t-}\right)=1$ for every $T_{i} \in\left[0, T^{\star}\right]$ given a $C_{t-} \in \mathcal{K} \backslash\{1\}$. Let us assume that the price process of a defaultable bond with credit migrations and fractional recovery should satisfy

$$
B\left(t, T_{i}, C_{t}\right)=\mathbb{E}_{\mathbb{Q}_{T_{i}}}\left(B\left(t, T_{i}, 0\right) 1_{\left\{\tau^{\star}>T_{i}\right\}}+q B^{\star} 1_{\left\{\tau^{\star} \leq T_{i}\right\}} \mid \mathcal{G}_{t}\right)
$$

where $\mathbb{Q}_{T_{i}}$ is the forward martingale measure for the date $T_{i}$, for every $i=0,1, \ldots, n$, with $0 \leq t \leq T_{i}$, and $q$ can be defined as the recovery rate or the fractional part of $B^{\star}$ that the investor will recover in case of default, such that $q \in[0,1]$. Notice that this structure of bond maturities is the time structure that mark the tenor structure in the LIBOR additive process.

3. Additionally, the value of the bond in case of default can be defined as

$$
B^{\star}:=\left\{\begin{array}{lll}
=B\left(\tau^{\star}, T_{i}, C_{\tau_{k-1}}\right) & \rightarrow & \text { market value } \\
=B\left(\tau^{\star}, T_{i}, 0\right) & \rightarrow & \text { treasury value } \\
=1 & \rightarrow & \text { par value }
\end{array}\right.
$$

4. On the other hand notice that if $C_{t-} \in \mathcal{K} \backslash\{1\}$, we shall interpret $B\left(t, T_{i}, C_{t-}\right)$ as the pre-default value of a $T_{i}$-maturity zero-coupon corporate bond, or more formally

$$
\begin{aligned}
B\left(t, T_{i}, C_{t-}\right) & =B\left(t, T_{i}, 0\right) \cdot \exp \left(-\int_{t}^{T_{j}} s\left(t, u, C_{t-}\right) d u\right) \\
& =B\left(t, T_{i}\right) \cdot S\left(t, T_{i}, C_{t-}\right)
\end{aligned}
$$

where $s\left(t, u, C_{t-}\right)$ is the instantaneous spread rate (see expression 2.14). 


\subsection{The building-blocks for Interest-Rate modelling}

Given the mentioned conditions in the previous section, here we characterize the functional forms of the dynamic of corporate bonds, when the LIBOR additive process is driving the forward rates dynamic, and more specifically, when we consider different frameworks in credit risk. Basically the term structure model is based on an exogenous specification of the dynamics of instantaneous, continuously compounded forward rates $f(t, T, c)$. Our aim in this section is to recover the functional form of corporate bonds from (2.10) for different frameworks of credit risk.

This section is organized as follows:

- Subsection 1 is devoted to develop the simplest case, the risk-free case, following closely Bjork et al. (1997) and Eberlein et al. (2006) for the Heath, Jarrow and Morton approach (1992), but now, introducing the LIBOR additive process.

- Basically the next subsections are extensions of the first one, in the sense that we include different credit risk frameworks for the corporate bond. Therefore, subsection 2 includes the credit risk but without the possibility to have credit migration between different rates.

- And it is in subsection 3 where we introduce the credit migration and we obtain specific functional forms for corporate bonds with these characteristics.

\subsubsection{Risk-free Bond Market Structure}

In this subsection, we introduce some well-known results due to Bjork, Di Masi, Kabanov and Runggaldier (1997) for risk-free bonds, that will be extended later for different credit-risk frameworks. Basically, here, we present the functional expression for the discounted default-free bond when the forward rates are driven by a LIBOR additive process.

According to the assumptions shown in section 2.1.4, it is easy to conclude that we have to consider a model of the dynamics of the default-free forward curve with the following SDE:

$$
\begin{aligned}
d f(t, T, 0) & =d f(t, T) \\
& =\alpha_{\eta(t)}(t, T) d t+\sigma_{\eta(t)}(t, T) d W_{t}+\int_{\mathbb{R}^{r}} \delta_{\eta(t)}(t, x, T)\left(v_{\eta(t)}-\mu_{\eta(t)}\right)(d t, d x)
\end{aligned}
$$

Basically, this model is an extension of the discretized Heath, Jarrow and Morton (1992) model, where the dynamic of forward rates include jumps, as appear in Bjork, Kabanov and Runggaldier (1997) and Bjork, Di Masi, Kabanov and Runggaldier (1997).

Henceforth, we can define the price of a discounted default-free zero-coupon bond as

$$
\begin{aligned}
Z\left(t, T_{i}\right) & =\frac{B\left(t, T_{i}\right)}{B_{t}} \\
& =\exp \left\{-\int_{0}^{t} r(s) d s-\int_{t}^{T_{i}} f(t, s) d s\right\}
\end{aligned}
$$

for any $0 \leq t \leq T_{i}$, with $T_{i} \in\left[0, T^{\star}\right]$, and $i=0,1, \ldots, n$. 
Proposition 52 The discounted bond price process $Z\left(t, T_{i}\right)$ has the form

$$
\begin{aligned}
Z\left(t, T_{i}\right)=Z\left(0, T_{i}\right) \exp \quad & \left\{\sum_{j \leq \eta(t)} \int_{T_{j}}^{t \wedge T_{j+1}} \tilde{a}_{j}\left(u, T_{i}\right) d u+\sum_{j \leq \eta(t)} \int_{T_{j}}^{t \wedge T_{j+1}} b_{j}\left(u, T_{i}\right) d W_{u}\right. \\
& \left.+\sum_{j \leq \eta(t)} \int_{T_{j}}^{t \wedge T_{j+1}} \int_{\mathbb{R}^{d}} h\left(u, x, T_{i}\right) \tilde{\mu}_{j}(d u, d x)\right\}
\end{aligned}
$$

and satisfies the linear stochastic differential equation

$$
\begin{aligned}
\frac{d Z\left(t, T_{i}\right)}{Z\left(t-, T_{i}\right)}= & \tilde{a}_{\eta(t)}\left(t, T_{i}\right) d t+b_{\eta(t)}\left(t, T_{i}\right) d W_{t}+\int_{\mathbb{R}^{d}} h\left(t, x, T_{i}\right) \tilde{\mu}_{\eta(t)}(d t, d x) \\
& +\int_{\mathbb{R}^{d}}\left(e^{h\left(t, x, T_{i}\right)}-1-h\left(t, x, T_{i}\right)\right) v_{\eta(t)}(d t, d x)
\end{aligned}
$$

with

$$
\tilde{a}_{\eta(t)}\left(t, T_{i}\right)=a_{\eta(t)}\left(t, T_{i}\right)+\frac{1}{2}\left|b_{\eta(t)}\left(t, T_{i}\right)\right|^{2}
$$

and

$$
\begin{aligned}
a_{\eta(t)}\left(t, T_{i}\right) & =-\sum_{\eta(t) \leq j \leq i-1} \int_{T_{j} \vee t}^{T_{j+1}} \alpha_{j}(t, s) d s \\
b_{\eta(t)}\left(t, T_{i}\right) & =-\sum_{\eta(t) \leq j \leq i-1} \int_{T_{j} \vee t}^{T_{j+1}} \sigma_{j}(t, s) d s \\
h\left(t, x, T_{i}\right) & =-\int_{t}^{T_{j}} \delta(t, x, s) d s
\end{aligned}
$$

Proof. This proof follows the same ideas as in Heath, Jarrow and Morton (1992), Brace, Gatarek and Musiela (1997), Bjork et al. (1997), Glasserman and Kou (1997) or Musiela and Rutkowski (2004).

Notice that according to the assumptions mentioned in section 2.1.3 we have

$$
\begin{aligned}
B\left(t, T_{i}\right)= & \exp \left\{-\int_{t}^{T_{i}} f(t, s) d s\right\} \\
= & \exp \left\{-\int_{t}^{T_{i}} f(0, s) d s\right. \\
& -\sum_{\eta(u) \leq j \leq i-1} \int_{T_{j} \vee t}^{T_{j+1}}\left(\sum_{j \leq \eta(t)} \int_{T_{j}}^{t \wedge T_{j+1}} \alpha_{j}(u, s) d u\right) d s \\
& -\sum_{\eta(u) \leq j \leq i-1} \int_{T_{j} \vee t}^{T_{j+1}}\left(\sum_{j \leq \eta(t)} \int_{T_{j}}^{t \wedge T_{j+1}} \sigma_{j}(u, s) d W_{u}\right) d s \\
& \left.-\sum_{\eta(u) \leq j \leq i-1} \int_{T_{j} \vee t}^{T_{j+1}}\left(\sum_{j \leq \eta(t)} \int_{T_{j}}^{t \wedge T_{j+1}} \int_{\mathbb{R}^{d}} \delta_{j}(u, x, s) 1_{\{|x| \leq 1\}} \tilde{\mu}_{j}(d u, d x)\right) d s\right\}
\end{aligned}
$$


whence using the stochastic version of Fubini's theorem (see Protter (1995) Theorem IV.4.45) we have

$$
\begin{aligned}
\ln B\left(t, T_{i}\right)= & -\int_{t}^{T_{i}} f(t, s) d s \\
= & -\int_{t}^{T_{i}} f(0, s) d s \\
& -\sum_{j \leq \eta(t)} \int_{T_{j}}^{t \wedge T_{j+1}}\left(\sum_{\eta(u) \leq j \leq i-1} \int_{T_{j} \vee t}^{T_{j+1}} \alpha_{j}(u, s) d s\right) d u \\
& -\sum_{j \leq \eta(t)} \int_{T_{j}}^{t \wedge T_{j+1}}\left(\sum_{\eta(u) \leq j \leq i-1} \int_{T_{j} \vee t}^{T_{j+1}} \sigma_{j}(u, s) d s\right) d W_{u} \\
& -\sum_{j \leq \eta(t)} \int_{T_{j}}^{t \wedge T_{j+1}} \int_{\mathbb{R}^{d}}\left(\sum_{\eta(u) \leq j \leq i-1} \int_{T_{j} \vee t}^{T_{j+1}} \delta_{j}(u, x, s) 1_{\{|x| \leq 1\}} d s\right) \tilde{\mu}_{j}(d u, d x)
\end{aligned}
$$

Splitting the integrals, we obtain

$$
\begin{aligned}
= & \int_{0}^{t} f(0, s) d s-\int_{0}^{T_{i}} f(0, s) d s \\
& +\sum_{j \leq \eta(t)} \int_{T_{j}}^{t \wedge T_{j+1}}\left(\sum_{\eta(u) \leq j \leq \eta(t)} \int_{T_{j} \vee u}^{T_{j+1} \wedge t} \alpha_{j}(u, s) d s-\sum_{\eta(u) \leq j \leq i-1} \int_{T_{j} \vee u}^{T_{j+1}} \alpha_{j}(u, s) d s\right) d u \\
& +\sum_{j \leq \eta(t)} \int_{T_{j}}^{t \wedge T_{j+1}}\left(\sum_{\eta(u) \leq j \leq \eta(t)} \int_{T_{j} \vee u}^{T_{j+1} \wedge t} \sigma_{j}(u, s) d s-\sum_{\eta(u) \leq j \leq i-1} \int_{T_{j} \vee u}^{T_{j+1}} \sigma_{j}(u, s) d s\right) d W_{u} \\
& +\sum_{j \leq \eta(t)} \int_{T_{j}}^{t \wedge T_{j+1}} \int_{\mathbb{R}^{d}}\left(\sum_{\eta(u) \leq j \leq \eta(t)} \int_{T_{j} \vee u}^{T_{j+1} \wedge t} \delta_{j}(u, x, s) 1_{\{|x| \leq 1\}} d s-\sum_{\eta(u) \leq j \leq i-1} \int_{T_{j} \vee u}^{T_{j+1}} \delta_{j}(u, x, s) 1_{\{|x| \leq 1\}} d s\right) \tilde{\mu}_{j}(d u, d x)
\end{aligned}
$$

For the sake of simplicity, let us rename

$$
\begin{aligned}
a_{j}\left(t, T_{i}\right) & :=-\sum_{\eta(t) \leq j \leq i-1} \int_{T_{j} \vee t}^{T_{j+1}} \alpha_{j}(t, s) d s \\
b_{j}\left(t, T_{i}\right): & :=-\sum_{\eta(t) \leq j \leq i-1} \int_{T_{j} \vee t}^{T_{j+1}} \sigma_{j}(t, s) d s \\
h\left(t, x, T_{i}\right): & :=-\int_{t}^{T_{j}} \delta(t, x, s) d s
\end{aligned}
$$

and notice that the sum of the four integrals in the left-hand side of the last equality coincides with the 
expression for the integrated short rate

$$
\begin{aligned}
\int_{0}^{t} r_{s} d s= & \int_{0}^{t} f(0, s) d s \\
& +\sum_{j \leq \eta(t)} \int_{T_{j}}^{t \wedge T_{j+1}}\left(\sum_{\eta(u) \leq j \leq i-1} \int_{T_{j} \vee u}^{T_{j+1}} \alpha_{j}(u, s) d u\right) d s \\
& +\sum_{j \leq \eta(t)} \int_{T_{j}}^{t \wedge T_{j+1}}\left(\sum_{\eta(u) \leq j \leq i-1} \int_{T_{j} \vee u}^{T_{j+1}} \sigma_{j}(u, s) d W_{u}\right) d s \\
& +\sum_{j \leq \eta(t)} \int_{T_{j}}^{t \wedge T_{j+1}}\left(\sum_{\eta(u) \leq j \leq i-1} \int_{T_{j} \vee u}^{T_{j+1}} \int_{X} \delta(u, x, s) 1_{\{|x| \leq 1\}} \tilde{\mu}_{j}(d u, d x)\right) d s
\end{aligned}
$$

Hence we obtain

$$
\begin{aligned}
\ln B\left(t, T_{i}\right)= & \ln B\left(0, T_{i}\right) \\
& +\sum_{j \leq \eta(t)} \int_{T_{j}}^{t \wedge T_{j+1}} a_{j}\left(u, T_{i}\right) d u \\
& +\sum_{j \leq \eta(t)} \int_{T_{j}}^{t \wedge T_{j+1}} b_{j}\left(t, T_{i}\right) d W_{u} \\
& +\sum_{j \leq \eta(t)} \int_{T_{j}}^{t \wedge T_{j+1}} \int_{\mathbb{R}^{d}} h\left(u, x, T_{i}\right) \tilde{\mu}_{j}(d u, d x) \\
& +\int_{0}^{t} r_{s} d s
\end{aligned}
$$

and this proves (2.19). By the Itô formula for semimartingales ${ }^{5}$, we have that

$$
\begin{aligned}
d Z\left(t, T_{i}\right)= & Z\left(t-, T_{i}\right)\left[a_{\eta(t)}\left(u, T_{i}\right) d t+\frac{1}{2}\left|b_{\eta(t)}\left(t, T_{i}\right)\right|^{2} d t+b_{\eta(t)}\left(t, T_{i}\right) d W_{t}\right. \\
& \left.+\int_{\mathbb{R}^{d}} h\left(u, x, T_{i}\right) 1_{\{|x| \leq 1\}} \tilde{\mu}_{\eta(t)}(d t, d x)+\int_{\mathbb{R}^{d}}\left(e^{h\left(u, x, T_{i}\right)}-1-h\left(u, x, T_{i}\right)\right) \mu_{\eta(t)}(d t, d x)\right]
\end{aligned}
$$

whence if we define $\tilde{a}_{\eta(t)}\left(u, T_{i}\right)=a_{\eta(t)}\left(u, T_{i}\right)+\frac{1}{2}\left|b_{\eta(t)}\left(t, T_{i}\right)\right|^{2},(2.20)$ follows.

\subsubsection{Conditional Corporate-Bond market structure}

In this subsection we introduce the credit risk for corporate bonds. Let us go one step further, including the credit rating $c \in \mathcal{K} \backslash\{1\}$ in the model of the dynamics of the instantaneous forward rate $f(t, T, c)$ in $t \leq T \in I$, using the form (2.10) under $\mathbb{P}$,

$$
d f(t, T, c)=\alpha_{\eta(t)}(t, T, c) d t+\sigma_{\eta(t)}(t, T, c) d W_{t}+\int_{\mathbb{R}^{r}} \delta(t, T, x)\left(\mu_{\eta(t)}^{c}-v_{\eta(t)}^{c}\right)(d t, d x)
$$

Additionally, assume that the price of a defaultable bond zero coupon bond with credit rate

\footnotetext{
${ }^{5}$ See Jacod and Shiryaev (1987) Ch.1 (4.57), or Cont and Tankov (2004) Ch. 8
} 
$C_{t_{-}} \in \mathcal{K} \backslash\{1\}$ can be expressed as

$$
B\left(t, T_{i}, C_{t_{-}}\right)=\exp \left\{-\int_{t}^{T_{i}} f\left(t, s, C_{t_{-}}\right) d s\right\}
$$

for any $0 \leq t \leq T_{i}$, with $T_{i} \in\left[t, T^{\star}\right]$, and $i=0,1, \ldots, n$. Notice that this value is the price of a corporate bond conditional that between $t$ and $T_{i}$ there is no possibility of credit migration. This is theoretically possible to define but impossible to find in the real world. However, it is worthy to develop this definition as a basic tool for the next section.

Theorem 53 For any $C_{t_{-}} \in \mathcal{K} \backslash\{1\}$, the discounted defaultable zero coupon bond price process $Z\left(t, T_{i}, C_{t_{-}}\right)$with $0 \leq t \leq T_{i} \leq T^{\star}$, has the form

$$
\begin{aligned}
Z\left(t, T_{i}, C_{t_{-}}\right)=Z\left(0, T_{i}, C_{t_{-}}\right) \exp & \left\{\sum_{j \leq \eta(t)} \int_{T_{j}}^{t \wedge T_{j+1}} \tilde{a}_{j}\left(u, T_{i}, C_{t_{-}}\right) d u+\sum_{j \leq \eta(t)} \int_{T_{j}}^{t \wedge T_{j+1}} b_{j}\left(u, T_{i}, C_{t_{-}}\right) d W_{u}\right. \\
& \left.+\sum_{j \leq \eta(t)} \int_{T_{j}}^{t \wedge T_{j+1}} \int_{\mathbb{R}^{d}} h\left(u, x, T_{i}\right) \tilde{\mu}_{j}^{C_{t_{-}}}(d u, d x)\right\}
\end{aligned}
$$

and satisfies the following linear stochastic differential equation

$$
\begin{aligned}
d\left(Z\left(t, T_{i}, C_{t_{-}}\right)\right)= & Z\left(t_{-}, T_{i}, C_{t_{-}}\right)\left[\tilde{a}_{\eta(t)}\left(t, T_{i}, C_{t_{-}}\right) d t+b_{\eta(t)}\left(t, T_{i}, C_{t_{-}}\right) d W_{t}\right. \\
& +\int_{\mathbb{R}^{d}} h\left(t, x, T_{i}\right) 1_{\{|x| \leq 1\}} \tilde{\mu}_{\eta(t)}^{C_{t_{-}}}(d t, d x) \\
& \left.+\int_{\mathbb{R}^{d}}\left(e^{h\left(t, x, T_{i}\right)}-1-h\left(t, x, T_{i}\right)\right) \mu_{\eta(t)}^{C_{t_{-}}}(d t, d x)\right]
\end{aligned}
$$

with

$$
\tilde{a}_{\eta(t)}\left(t, T_{i}, C_{t_{-}}\right):=a_{\eta(t)}\left(t, T_{i}, C_{t_{-}}\right)+\frac{1}{2}\left|b_{\eta(t)}\left(t, T_{i}, C_{t_{-}}\right)\right|^{2}+s\left(t, T_{i}, C_{t_{-}}\right)
$$

and

$$
\begin{aligned}
a_{\eta(t)}\left(t, T_{i}, C_{t_{-}}\right) & :=-\sum_{\eta(t) \leq j \leq i-1} \int_{T_{j} \vee t}^{T_{j+1}} \alpha_{j}\left(t, s, C_{t_{-}}\right) d s \\
b_{\eta(t)}\left(t, T_{i}, C_{t_{-}}\right) & :=-\sum_{\eta(t) \leq j \leq i-1} \int_{T_{j} \vee t}^{T_{j+1}} \sigma_{j}\left(t, s, C_{t_{-}}\right) d s \\
h\left(t, x, T_{i}\right) & : \quad=-\int_{u}^{T_{i}} \delta(t, x, s) d s
\end{aligned}
$$


Proof. Following the similar procedure as in the proof of Proposition 52 we obtain

$$
\begin{aligned}
\ln B\left(t, T_{i}, C_{t_{-}}\right)= & -\int_{0}^{T_{i}} f\left(0, s, C_{t_{-}}\right) d s \\
& +\sum_{j \leq \eta(t)} \int_{T_{j}}^{t \wedge T_{j+1}} a_{j}\left(u, T_{i}, C_{t_{-}}\right) d u \\
& +\sum_{j \leq \eta(t)} \int_{T_{j}}^{t \wedge T_{j+1}} b_{j}\left(u, T_{i}, C_{t_{-}}\right) d W_{u} \\
& +\sum_{j \leq \eta(t)} \int_{T_{j}}^{t \wedge T_{j+1}} \int_{\mathbb{R}^{d}} h\left(u, x, T_{i}\right) \tilde{\mu}_{j}^{C_{t_{-}}}(d u, d x) \\
& +\sum_{j \leq \eta(t)} \int_{T_{j}}^{t \wedge T_{j+1}} r_{s}^{C_{t_{-}}} d s
\end{aligned}
$$

Additionally if we decompose the defaultable short rate into the risk-free short-rate and short term credit spread such that

$$
\int_{0}^{t} r_{t}^{C_{t_{-}}} d t=\int_{0}^{t} r_{t}^{0} d t+\int_{0}^{t} s\left(t, t, C_{t_{-}}\right) d t
$$

then this proves (2.24). By the Itô formula for semimartingales ${ }^{6}$, we get from that

$$
\begin{aligned}
d Z\left(t, T_{i}, C_{t_{-}}\right)= & Z\left(t, T_{i}, C_{t_{-}}\right)\left[\left(a_{\eta(t)}\left(t, T_{i}, C_{t_{-}}\right)+s\left(t, t, C_{t_{-}}\right)+\frac{1}{2}\left|b_{\eta(t)}\left(t, T_{i}, C_{t_{-}}\right)\right|^{2}\right) d t\right. \\
& +b_{\eta(t)}\left(t, T_{i}, C_{t_{-}}\right) d W_{t} \\
& +\int_{\mathbb{R}^{d}} h\left(t, x, T_{i}\right) 1_{\{|x| \leq 1\}} \tilde{\mu}_{\eta(t)}^{C_{t_{-}}}(d t, d x) \\
& \left.+\int_{\mathbb{R}^{d}}\left(e^{h\left(t, x, T_{i}\right)}-1-h\left(t, x, T_{i}\right)\right) \mu_{\eta(t)}^{C_{t_{-}}}(d t, d x)\right]
\end{aligned}
$$

If we define $\tilde{a}_{\eta(t)}\left(t, T_{i}, C_{t_{-}}\right):=a_{\eta(t)}\left(t, T_{i}, C_{t_{-}}\right)+s\left(t, t, C_{t_{-}}\right)+\frac{1}{2}\left|b_{\eta(t)}\left(t, T_{i}, C_{t_{-}}\right)\right|^{2}$ then (2.25) holds.

\subsubsection{Corporate-Bond Market Structure with Credit Migration and Default}

This subsection is devoted to expose the dynamics of the corporate-bond prices with credit migration, and different structures of recovery in case of default (see assumptions for the credit risk model). Basically here we consider that the price process of a defaultable bond with credit migrations and fractional recovery should satisfy

$$
B\left(t, T_{i}, C_{t}\right)=\mathbb{E}_{\mathbb{Q}_{T_{i}}}\left(B\left(t, T_{i}, 0\right) 1_{\left\{\tau^{\star}>T_{i}\right\}}+q B^{\star} 1_{\left\{\tau^{\star} \leq T_{i}\right\}} \mid \mathcal{G}_{t}\right)
$$

where $\mathbb{Q}_{T_{j}}$ is the forward martingale measure for the date $T_{i}$ for every $i=0,1, \ldots, n$, with $0 \leq t \leq T_{i}$, where $q \in[0,1]$ represents the fractional part of $B^{\star}$ that the investor will recover in case of default (recovery rate). More specifically, here we will consider three possible cases

$$
B^{\star}=\left\{\begin{array}{lll}
=B\left(\tau^{\star}, T_{i}, C_{\tau_{k-1}}\right) & \rightarrow \text { market value } \\
=B\left(\tau^{\star}, T_{i}, 0\right) & \rightarrow \text { treasury value } \\
=1 & \rightarrow \text { par value }
\end{array}\right.
$$

\footnotetext{
Ch. 8

${ }^{6}$ See Jacod and Shiryaev (1987) Ch.1 (4.57), Goll and Kallsen (2000) Lemma A.5, or Cont and Tankov (2004)
} 
In the first case, the investor only can recover a fraction of the market value of the bond just quoted in the moment prior to default. In the second case, we consider the recovery in case of a default, of a fractional part of a different bond, usual a risk-free or treasury bond, and finally, a fractional recovery of the par value of the bond.

\section{Corporate-bond dynamics with fractional recovery of market value}

Consider the price of a defaultable zero-coupon bond with a given recovery rate $^{7}$, i.e.,

$$
B\left(t, T_{i}, C_{t}\right)=\mathbb{E}_{\mathbb{Q}_{T_{i}}}\left(B\left(t, T_{i}, 0\right) 1_{\left\{\tau^{\star}>T_{i}\right\}}+q B\left(\tau^{\star}, T_{i}, C_{\tau_{k-1}}\right) 1_{\left\{\tau^{\star} \leq T_{i}\right\}} \mid \mathcal{G}_{t}\right)
$$

where $\mathbb{Q}_{T_{i}}$ is the forward martingale measure for the date $T_{i}$ for every $i=0,1, \ldots, n$, with $0 \leq t \leq T_{i}, k \in$ $\mathbb{N}_{+}$, and $C_{t} \in \mathcal{K} \backslash\{1\}$ with

$$
C_{t}=C_{0}+\sum_{a, b \in \mathcal{K}} \int_{0}^{t}(b-a) 1_{\left\{C_{s-}=a\right\}} d N_{a b}(s)
$$

where $C_{t-} \in \mathcal{K} \backslash\{1\}$ is the credit rate in the prior to the moment of jump in $t$ (see assumptions in section 2.1.3).

We define the price of a discounted defaultable zero coupon bond as

$$
\begin{aligned}
Z\left(t, T_{i}, C_{t}\right) & =\frac{B\left(t, T_{i}, C_{t}\right)}{B_{t}} \\
& =\mathbb{E}_{\mathbb{Q}_{T_{i}}}\left(Z\left(t, T_{i}, 0\right) 1_{\left\{\tau^{\star}>T_{i}\right\}}+q Z\left(\tau^{\star}, T_{i}, C_{\tau_{k-1}}\right) 1_{\left\{\tau^{\star} \leq T_{i}\right\}} \mid \mathcal{G}_{t}\right)
\end{aligned}
$$

Proposition 54 For any $C_{t-} \in \mathcal{K} \backslash\{1\}$, the discounted defaultable zero coupon bond price process $Z\left(t, T_{i}, C_{t}\right)$ with fractional recovery $q$ of market value on $\left[0, T_{i}\right]$ satisfies the following linear stochastic differential equation

$$
d Z\left(t, T_{i}, C_{t}\right)=d Z\left(t, T_{i}, C_{t-}\right)-l \cdot Z\left(t, T_{i}, C_{t-}\right) \cdot d\left(1_{\left\{C_{t}=1\right\}}\right)
$$

where $l$ is the loss rate $l=1-q$.

Proof. Under the fractional-recovery of market value hypothesis ${ }^{8}$, since $1_{\left\{C_{t}=h\right\}}$ is a process of finite variation, for any $h=0, . ., 1-\frac{1}{m}, 1$, with $m \in \mathbb{N}^{+}$, therefore, an application of Itô's rule yields

$$
d Z\left(t, T_{i}, C_{t}\right)=\sum_{h=0}^{1-\frac{1}{m}}\left[d Z\left(t, T_{i}, C_{t-}\right) 1_{\left\{C_{t-}=h\right\}}+Z\left(t, T_{i}, C_{t-}\right) d\left(1_{\left\{C_{t}=h\right\}}\right)\right]+Z\left(t, T_{i}, C_{t-}\right) \cdot q \cdot d\left(1_{\left\{C_{t}=1\right\}}\right)
$$

Notice that $C_{t}$ is a $\mathcal{C}_{t}$-adapted process. Therefore

$$
\sum_{h=0}^{1-\frac{1}{m}} d Z\left(t, T_{i}, C_{t-}\right) 1_{\left\{C_{t-}=h\right\}}=d Z\left(t, T_{i}, c\right)
$$

\footnotetext{
${ }^{7}$ See details in Schönbucher (2003)

${ }^{8}$ See Duffie and Singleton (1999) for an extensive mathematical work of valuation under a "recovery of market value" framework.
} 
and

$$
\begin{aligned}
\sum_{h=0}^{1-\frac{1}{m}} Z\left(t, T_{i}, C_{t-}\right) d\left(1_{\left\{C_{t}=h\right\}}\right) & =Z\left(t, T_{i}, c\right) \sum_{h=0}^{1-\frac{1}{m}} d\left(1_{\left\{C_{t}=h\right\}}\right) \\
& =-Z\left(t, T_{i}, c\right) d\left(1_{\left\{C_{t}=1\right\}}\right)
\end{aligned}
$$

using the fact that $\sum_{i=0}^{1-\frac{1}{m}} 1_{\left\{C_{t}=i\right\}}=1-1_{\left\{C_{t}=1\right\}}$ whence $\sum_{i=0}^{1-\frac{1}{m}} d\left(1_{\left\{C_{t}=i\right\}}\right)=-d\left(1_{\left\{C_{t}=1\right\}}\right)$.

As a direct result, we have the expression

$$
d Z\left(t, T_{i}, C_{t}\right)=d Z\left(t, T_{i}, c\right)+(q-1) Z\left(t, T_{i}, c\right) d\left(1_{\left\{C_{t}=1\right\}}\right)
$$

and taking into account that $l=1-q$, we proved $(2.28)$.

Theorem 55 For any $C_{t-} \in \mathcal{K} \backslash\{1\}$, the discounted defaultable zero coupon bond price process $Z\left(t, T_{i}, c\right)$ on $\left[0, T_{i}\right]$ follows

$$
\begin{aligned}
\frac{d Z\left(t, T_{i}, C_{t}\right)}{Z\left(t_{-}, T_{i}, C_{t-}\right)}= & \left(a_{j}\left(t, T_{i}, c\right)+s(t, t, c)+\frac{1}{2}\left|b_{j}\left(t, T_{i}, c\right)\right|^{2}\right) d t \\
& +b_{j}\left(t, T_{i}, c\right) d W_{t} \\
& +\int_{\mathbb{R}^{d}} h\left(t, x, T_{i}\right) 1_{\{|x| \leq 1\}} \tilde{\mu}_{j}^{c}(d t, d x) \\
& +\int_{\mathbb{R}^{d}}\left(e^{h\left(t, x, T_{i}\right)}-1-h\left(t, x, T_{i}\right)\right) \mu_{j}^{c}(d t, d x) \\
& -l \cdot d\left(1_{\left\{C_{\tau_{k}}=1\right\}}\right)
\end{aligned}
$$

Proof. Directly, using Theorem 53 and Proposition 54.

\section{Corporate-bond dynamics with fractional recovery of treasury}

In this second case, we are assuming that under this model, the issuer of the corporate bond, in the default case, will pay a fractional part of a risk-free bond with identical maturity, such that

$$
B\left(t, T_{i}, C_{t}\right)=\mathbb{E}_{\mathbb{Q}_{T_{i}}}\left(B\left(t, T_{i}, 0\right) 1_{\left\{\tau^{\star}>T_{i}\right\}}+q B\left(\tau^{\star}, T_{i}, 0\right) 1_{\left\{\tau^{\star} \leq T_{i}\right\}} \mid \mathcal{G}_{t}\right)
$$

Proposition 56 For any $C_{t-} \in \mathcal{K} \backslash\{1\}$, the discounted defaultable zero coupon bond price process $Z\left(t, T_{i}, C_{t}\right)$ with fractional recovery $q$ of treasury-bond value on $\left[0, T_{i}\right]$ satisfies the following linear stochastic differential equation

$$
\begin{aligned}
\frac{d Z\left(t, T_{i}, C_{t}\right)}{Z\left(t_{-}, T_{i}, C_{t-}\right)}= & \left(a_{\eta(t)}\left(t, T_{i}, C_{t-}\right)+s\left(t, t, C_{t-}\right)+\frac{1}{2}\left|b_{\eta(t)}\left(t, T_{i}, C_{t-}\right)\right|^{2}\right) d t \\
& +b_{\eta(t)}\left(t, T_{i}, C_{t-}\right) d W_{t} \\
& +\int_{\mathbb{R}^{d}} h\left(t, x, T_{i}\right) 1_{\{|x| \leq 1\}} \tilde{\mu}_{\eta(t)}^{C_{t-}}(d t, d x) \\
& +\int_{\mathbb{R}^{d}}\left(e^{h\left(t, x, T_{i}\right)}-1-h\left(t, x, T_{i}\right)\right) \mu_{\eta(t)}^{C_{t-}}(d t, d x) \\
& -\left(1-\frac{q}{S\left(t, T_{i}, C_{t-}\right)}\right) \cdot d\left(1_{\left\{C_{\tau_{k}}=1\right\}}\right)
\end{aligned}
$$


where $S\left(t, T_{i}, C_{t-}\right)=\exp \left\{-\int_{t}^{T_{j}} s\left(t, s, C_{t-}\right) d s\right\}$.

Proof. In this case, under the fractional recovery of treasury hypothesis, we have

$$
\begin{aligned}
d Z\left(t, T_{i}, C_{t}\right)= & \sum_{h=0}^{1-\frac{1}{m}}\left[d Z\left(t, T_{i}, C_{t-}\right) 1_{\left\{C_{t-}=h\right\}}+Z\left(t, T_{i}, C_{t-}\right) d\left(1_{\left\{C_{t}=h\right\}}\right)\right] \\
& +B\left(t, T_{i}, 0\right) \cdot q \cdot d\left(1_{\left\{C_{t}=1\right\}}\right)
\end{aligned}
$$

where if we set $j=\eta(t)$ and $c=C_{t-} \in \mathcal{K} \backslash\{1\}$, and using the following expressions (see Proposition 54)

$$
\sum_{h=0}^{1-\frac{1}{m}} d Z\left(t, T_{i}, C_{t-}\right) 1_{\left\{C_{t-}=h\right\}}=d Z\left(t, T_{i}, c\right)
$$

and

$$
\begin{aligned}
\sum_{h=0}^{1-\frac{1}{m}} Z\left(t, T_{i}, C_{t-}\right) d\left(1_{\left\{C_{t}=h\right\}}\right) & =Z\left(t, T_{i}, c\right) \sum_{h=0}^{1-\frac{1}{m}} d\left(1_{\left\{C_{t}=h\right\}}\right) \\
& =-Z\left(t, T_{i}, c\right) d\left(1_{\left\{C_{t}=1\right\}}\right)
\end{aligned}
$$

We obtain the assertion upon using the fact that $\sum_{i=0}^{1-\frac{1}{m}} 1_{\left\{C_{t}=i\right\}}=1-1_{\left\{C_{t}=1\right\}}: \sum_{i=0}^{1-\frac{1}{m}} d\left(1_{\left\{C_{t}=i\right\}}\right)=$ $-d\left(1_{\left\{C_{t}=1\right\}}\right)$.

Therefore we obtain the following expression

$$
\begin{aligned}
d Z\left(t, T_{i}, C_{t}\right)= & d Z\left(t, T_{i}, c\right)-Z\left(t, T_{i}, c\right) d\left(1_{\left\{C_{t}=1\right\}}\right)+\frac{Z\left(t, T_{i}, c\right)}{S\left(t, T_{i}, c\right)} \cdot q \cdot d\left(1_{\left\{C_{t}=1\right\}}\right) \\
= & Z\left(t, T_{i}, c\right)\left[\left(a_{j}\left(t, T_{i}, c\right)+s(t, t, c)+\frac{1}{2}\left|b_{j}\left(t, T_{i}, c\right)\right|^{2}\right) d t\right. \\
& +b_{j}\left(t, T_{i}, c\right) d W_{t}+\int_{\mathbb{R}^{d}} h\left(t, x, T_{i}\right) 1_{\{|x| \leq 1\}} \tilde{\mu}_{j}^{c}(d t, d x) \\
& \left.+\int_{\mathbb{R}^{d}}\left(e^{h\left(t, x, T_{i}\right)}-1-h\left(t, x, T_{i}\right)\right) \mu_{j}^{c}(d t, d x)\right] \\
& -Z\left(t, T_{i}, c\right)\left(1-\frac{q}{S\left(t, T_{i}, c\right)}\right) d\left(1_{\left\{C_{t}=1\right\}}\right)
\end{aligned}
$$

\section{Corporate-bond dynamics with fractional recovery of par value}

Finally, we assume that the issuer of the corporate bond, in the default case, will pay a fractional part of the par value, such that

$$
B\left(t, T_{j}, C_{t}\right)=\mathbb{E}_{\mathbb{Q}_{T_{j}}}\left(B\left(t, T_{j}, 0\right) 1_{\left\{\tau^{\star}>T_{j}\right\}}+q 1_{\left\{\tau^{\star} \leq T_{j}\right\}} \mid \mathcal{G}_{t}\right)
$$

Proposition 57 For any $C_{t-} \in \mathcal{K} \backslash\{1\}$, the discounted defaultable zero coupon bond price process $Z\left(t, T_{i}, C_{t}\right)$ with fractional recovery $q$ of par value on $\left[0, T_{i}\right]$ satisfies the following linear stochastic 
differential equation

$$
\begin{aligned}
\frac{d Z\left(t, T_{i}, C_{t}\right)}{Z\left(t_{-}, T_{i}, C_{t-}\right)}= & \left(a_{\eta(t)}\left(t, T_{i}, C_{t-}\right)+s(t, t, c)+\frac{1}{2}\left|b_{\eta(t)}\left(t, T_{i}, C_{t-}\right)\right|^{2}\right) d t \\
& +b_{\eta(t)}\left(t, T_{i}, C_{t-}\right) d W_{t}+\int_{\mathbb{R}^{d}} h\left(t, x, T_{i}\right) 1_{\{|x| \leq 1\}} \tilde{\mu}_{\eta(t)}^{C_{t-}}(d t, d x) \\
& +\int_{\mathbb{R}^{d}}\left(e^{h\left(t, x, T_{i}\right)}-1-h\left(t, x, T_{i}\right)\right) \mu_{\eta(t)}^{C_{t-}}(d t, d x) \\
& -\left(1-\frac{q}{Z\left(t, T_{i}, C_{t-}\right)}\right) \cdot d\left(1_{\left\{C_{\tau_{k}}=1\right\}}\right)
\end{aligned}
$$

Proof. Similar to the previous Proposition 56. 


\subsection{Absence of arbitrage and dynamics under a martingale mea- sure}

Let us recall that we are given a stochastic basis $(\Omega, \mathbb{F}, \mathbb{P})$ where $\mathbb{P}$ can be interpreted as the real-world probability measure and the original filtration $\mathbb{F}=\left(\mathcal{F}_{t}\right)$ with respect to which all processes are adapted; it is the filtration generated by $G$ and $C$.

Let us define $\mathcal{Q}_{\mathcal{F}}$ as the set of all probability measures $\tilde{\mathbb{P}}$ with $\tilde{\mathbb{P}}_{\mathcal{F}_{t}} \backsim \mathbb{P}_{\mathcal{F}_{t}}$ for all finite $t$ such that all the discounted zero-coupon bond prices process, $Z\left(t, T, C_{t}\right)$, are local $\tilde{\mathbb{P}}$ - martingales for every $T_{i} \in J$ and relative to $\left(\mathcal{G}_{t}\right)$, or in other words

$$
\begin{aligned}
\mathcal{Q}_{\mathcal{F}}: & =\left\{\tilde{\mathbb{P}} \in \mathcal{M}^{1}(\Omega, \mathcal{G}): \tilde{\mathbb{P}}_{\mathcal{G}_{t}} \backsim \mathbb{P}_{\mathcal{G}_{t}} \text { and }\left(Z\left(t, T, C_{t}\right)\right)_{0 \leq t \leq T}\right. \\
& \text { for } \left.C_{t} \in \mathcal{K}=\left\{0, \frac{1}{m}, \frac{2}{m}, \ldots, \frac{m-1}{m}, 1\right\} \text { is a local } \tilde{\mathbb{P}} \text {-martingale for any } 0 \leq t \leq T \in\left[0, T^{\star}\right]\right\}
\end{aligned}
$$

where $\mathcal{M}^{1}(\Omega, \mathcal{G})$ denotes the set of all probability measures on the measurable space $(\Omega, \mathcal{G})$.

We say that a model admits the existence of an equivalent martingale measure property (EMM) if the set $\mathcal{Q}_{\mathcal{F}}$ is non empty, and the economy represented by this model is complete if this martingale measure is unique. Then two questions naturally arise:

1. Can our model be an equilibrium or no-arbitrage model? Or equivalently: Can we find the martingale measure using our model? The answer is "no" unless we have a very special structure for the coefficients of our model. The present section is devoted to show these conditions under different credit risk frameworks.

2. Assuming that there exists an equivalent martingale measure, Is our model complete? or in other words, Is this martingale measure unique? The answer is "no", even if the dimension of the LIBOR market process is one (see Eberlein et al. (2006)) due to the introduction of the credit migration.

This section is basically focused to derive the necessary and sufficient conditions on the forward rate process with credit risk, such that there exists an equivalent martingale measure according to the wellknown theorems of asset-pricing that appear in Harrison and Kreps (1979) and Harrison and Pliska (1981). Basically we generalize the corresponding results of Heath, Jarrow and Morton (1992) and Björk et al. (1997) and we obtain the no-arbitrage expressions for different frameworks of credit risk and we derive a new risk-neutral form for the probability of default. An outline of this section is as follows:

- In subsection 1 we mainly focus to obtain the no-arbitrage conditions when we assume corporate bonds with credit migration and fractional recovery of market value. Under this framework we obtain the necessary conditions to have a discounted corporate bond martingale, and we derive some relevant results and expressions for the forward rate process and probability of default.

- In subsection 2 we derive similar results but when we assume corporate bonds with credit migration and fractional recovery of treasury.

- And identically, in subsection 3 we study how to obtain the equivalent martingale measure in the case of corporate bonds with credit migration and fractional recovery of par value.

In order to construct this set $\mathcal{Q}_{\mathcal{F}}$ we will follow Jacod and Shiryaev (1989), Björk et al. (1997) and Eberlein et al. (2006). Let us consider the sequence of pairs $\left(\beta_{j}, Y_{j}\right)$ such that 
- $\beta_{j}=\left(\beta_{j}^{i^{\prime}}(t)\right)_{i^{\prime} \leq d}$ is a predictable $\mathbb{R}^{d}$-valued process such that

$$
\sum_{j \leq \eta(t)} \int_{T_{j}}^{t \wedge T_{j+1}}\left(\beta_{j}^{\prime} A_{j} \beta_{j}\right) d s<\infty: \text { for any } t \in\left[0, T^{\star}\right] \text { a.s. }
$$

- $Y_{j}=\left(Y_{j}(\omega, t, x)\right)$ is a sequence of $\mathcal{R}^{d}$-measurable $(0, \infty)$-valued function such that

$$
\sum_{j \leq \eta(t)} \int_{T_{j}}^{t \wedge T_{j+1}} \int_{\mathbb{R}^{d}}\left(Y_{j}(s, x)-1\right) d v_{\eta(t)}(d x, d s)<\infty: \text { for } t \in\left[0, T^{\star}\right] \text { a.s. }
$$

Using these definitions, let us formulate a modified 'short' version of Girsanov's Theorem for semimartingales ${ }^{9}$

Theorem 58 Let the sequence of pairs $\left(\beta_{j}, Y_{j}\right)_{j=0,1, \ldots, n}$ be defined as above, and let us define the density process $M$ by

$$
d M_{t}=M_{t} \beta_{\eta(t)} d W_{t}+M_{t-} \int_{\mathbb{R}^{d}}\left(Y_{\eta(t)}(t, x)-1\right)\left(\mu_{\eta(t)}-v_{\eta(t)}\right)(d t, d x)
$$

with $M_{0}=1$ and suppose that for all finite $t$

$$
\mathbb{E}^{\mathbb{P}}\left[M_{t}\right]=1
$$

Then there exists a probability measure $\tilde{\mathbb{P}}$ on $\mathcal{F}$ locally equivalent to $\mathbb{P}$ with

$$
d \tilde{\mathbb{P}}_{t}=M_{t} d \mathbb{P}_{t}
$$

such that:

(i) $\tilde{W}_{t}:=W_{t}-\sum_{j \leq \eta(t)} \int_{T_{j}}^{t \wedge T_{j+1}} \beta_{j}(s) d s$ is a $\tilde{\mathbb{P}}$-Wiener process, and

(ii) $v_{\eta(t)}^{\tilde{\mathbb{P}}}(t, d x)=Y_{\eta(t)}(t, x) \cdot v_{\eta(t)}(t, d x)$ is the $\tilde{\mathbb{P}}$-compensator of $\mu_{\eta(t)}$.

Remark 59 Notice that the real-world probability measure $\mathbb{P}$ itself belongs to $\mathcal{Q}_{\mathcal{F}}$ if we use directly as Girsanov's quantities $\left(\beta_{j}=0, Y_{j}=1\right)$ for any $j=0,1, \ldots, n$, (see Example 51 in Chapter 1$)$.

In the following three subsections, for sake of clarity, we assume directly that $\mathbb{P} \in \mathcal{Q}_{\mathcal{F}}$ or equivalently, using the Girsanov's quantities $\left(\beta_{j}=0, Y_{j}=1\right)$ for any $j=0,1, \ldots, n$.

\subsubsection{Absence of arbitrage condition in a corporate-bond market with frac- tional recovery of market value}

Basically this subsection is devoted to show the main results concerning the existence of an equivalent martingale measure when we assume credit migration with fractional recovery of market value. They generalize the corresponding results of Heath, Jarrow and Morton (1992) and Björk et al. (1997). Let us recall that a model has the equivalent martingale measure property $(\mathrm{EMM})$ if the set $\mathcal{Q}_{\mathcal{F}}$ is not empty.

\footnotetext{
${ }^{9}$ The reader can find an extended and complete version of this Girsanov Theorem for Semimartingales in the Chapter 1 of this thesis (Theorem 48).
} 
Proposition 60 The initial probability measure $\mathbb{P}$ itself belongs to $\mathcal{Q}_{\mathcal{F}}$ if and only if the following two conditions hold, for every $T_{i} \in J$ :

$$
\sum_{j \leq \eta(t)} \int_{T_{j}}^{t \wedge T_{j+1}} \int_{\mathbb{R}^{d}}\left(e^{h\left(t, x, T_{i}\right)}-1-h\left(t, x, T_{i}\right)\right) \mu_{j}^{C_{t}}(d s, d x)<\infty
$$

and

$$
\tilde{a}_{\eta(t)}\left(t, T_{i}, C_{t-}\right)-l \cdot \lambda_{c_{t_{-}, 1}}+\int_{\mathbb{R}^{d}}\left(e^{h\left(t, x, T_{i}\right)}-1-h\left(t, x, T_{i}\right)\right) \mu_{\eta(t)}^{C_{t_{-}}}(d t, d x)=0
$$

for any $t \in\left[0, T_{i}\right]$, and any $C_{t_{-}} \in \mathcal{K} \backslash\{1\}$ where ${ }^{10}$

$$
\tilde{a}_{\eta(t)}\left(t, T_{i}, C_{t-}\right):=a_{\eta(t)}\left(t, T_{i}, C_{t-}\right)+s\left(t, t, C_{t-}\right)+\frac{1}{2}\left|b_{\eta(t)}\left(t, T_{i}, C_{t-}\right)\right|^{2}
$$

Proof. $[\Leftarrow]$ According to Theorem 55 and assuming, for the sake of simplicity, that $j=\eta(t)$

$$
\begin{aligned}
\frac{d Z\left(t, T_{i}, C_{t}\right)}{Z\left(t_{-}, T_{i}, C_{t-}\right)}= & \left(a_{j}\left(t, T_{i}, c\right)+s(t, t, c)+\frac{1}{2}\left|b_{j}\left(t, T_{i}, c\right)\right|^{2}\right) d t \\
& +b_{j}\left(t, T_{i}, c\right) d W_{t} \\
& +\int_{\mathbb{R}^{d}} h\left(t, x, T_{i}\right) 1_{\{|x| \leq 1\}} \tilde{\mu}_{j}^{c}(d t, d x) \\
& +\int_{\mathbb{R}^{d}}\left(e^{h\left(t, x, T_{i}\right)}-1-h\left(t, x, T_{i}\right)\right) \mu_{j}^{c}(d t, d x) \\
& -l \cdot d\left(1_{\left\{C_{\tau_{k}}=1\right\}}\right)
\end{aligned}
$$

Notice that using the Doob-Meyer expression

$$
d\left(1_{\left\{C_{t}=1\right\}}\right)=d M_{1}(t)+\lambda_{C_{t_{-}, 1}} d t
$$

we get

$$
\begin{aligned}
\frac{d Z\left(t, T_{i}, C_{t}\right)}{Z\left(t_{-}, T_{i}, C_{t-}\right)}= & \left(\tilde{a}_{j}\left(t, T_{i}, c\right)-l \cdot \lambda_{C_{t_{-}}, 1}\right) d t \\
& +b_{j}\left(t, T_{i}, c\right) d W_{t} \\
& +\int_{\mathbb{R}^{d}} h\left(t, x, T_{i}\right) 1_{\{|x| \leq 1\}} \tilde{\mu}_{j}^{c}(d t, d x) \\
& +\int_{\mathbb{R}^{d}}\left(e^{h\left(t, x, T_{i}\right)}-1-h\left(t, x, T_{i}\right)\right) \mu_{j}^{c}(d t, d x) \\
& -l \cdot d M_{1}(t)
\end{aligned}
$$

which has a local martingale solution if

$$
\left(\tilde{a}_{j}\left(t, T_{i}, c\right)-l \cdot \lambda_{C_{t_{-}, 1}}\right) d t+\int_{\mathbb{R}^{d}}\left(e^{h\left(t, x, T_{i}\right)}-1-h\left(t, x, T_{i}\right)\right) \mu_{j}^{c}(d t, d x)=0
$$

and

$$
\int_{\mathbb{R}^{d}}\left(e^{h\left(t, x, T_{i}\right)}-1-h\left(t, x, T_{i}\right)\right) \mu_{j}^{c}(d t, d x)<\infty
$$

$[\Rightarrow]$ Let us define the process $M:=\left[Z\left(t_{-}, T_{i}, C_{\tau_{k}}\right)\right]^{-1} Z\left(t, T_{i}, C_{t}\right)$ that is a local martingale. Let $\mu^{M}$ be the jump measure of $M$, and $v^{M}$ be its compensator. According to Jacod and Shiryaev (1989)

\footnotetext{
${ }^{10}$ Notice that if $C_{\tau_{k}}=0$ it means default-free asset and $s_{t}^{0}\left(T_{i}\right)=0$. It is the risk-free bond.
} 
(II.2.29) we have that $\int_{\mathbb{R}^{d}}|x| \wedge|x|^{2} v_{i}^{M}(d x)<\infty$ for finite $t$. Hence, for every $j=0,1, \ldots, n$

$$
\int_{\mathbb{R}^{d}}\left(\left|e^{h\left(t, x, T_{i}\right)}-1\right| \wedge\left|e^{h\left(t, x, T_{i}\right)}-1\right|^{2}\right) v_{j}^{M}(d x)=\int_{\mathbb{R}^{d}}\left(|x| \wedge|x|^{2}\right) v_{j}^{M}(d x)<\infty
$$

Since $\int_{\mathbb{R}^{d}}\left|h\left(t, x, T_{i}\right)\right|^{2} v_{t}(d x)<\infty$ the first condition holds, by virtue of the following inequality

$$
e^{h\left(t, x, T_{i}\right)}-1-h\left(t, x, T_{i}\right) \leq C\left(\left|e^{h\left(t, x, T_{i}\right)}-1\right| \wedge\left|e^{h\left(t, x, T_{i}\right)}-1\right|^{2}+h\left(t, x, T_{i}\right)^{2}\right)
$$

where $C$ is a constant. Using the dynamic of $\left[Z\left(t_{-}, T_{i}, C_{t_{-}}\right)\right]^{-1} Z\left(t, T_{i}, C_{t}\right)$, we infer that $M$ is a local martingale only if the process given by the left hand side is equal to zero.

Remark 61 This is a generalization of the Heath, Jarrow and Morton (1992) drift condition when the credit migration and default are possible. It reveals that in a simple remarkable way, this model can be specified under a (local) martingale measure.

Remark 62 Notice that under this framework, the risk-neutral condition has a direct relationship with the intensity matrix or with the default probabilities in the following sense:

$$
\lambda_{c_{t_{-}, 1}}=\frac{1}{(1-q)}\left[\tilde{a}_{j}\left(t, T_{i}, C_{t-}\right)+\int_{\mathbb{R}^{d}}\left(e^{h\left(t, x, T_{i}\right)}-1-h\left(t, x, T_{i}\right)\right) \mu_{j}^{C^{t_{-}}}(d t, d x)\right]
$$

Additionally, we can obtain, almost directly, the following results:

- The first one (Proposition 63) is related with the risk-neutral dynamics of instantaneous forward rates, and this result will be invariant with respect to the recovery framework we use.

- The second proposition (Proposition 64) basically exposes the dynamics of corporate-bonds with fractional recovery of market value when we impose the risk-neutrality using conditions (2.34) and (2.35).

Proposition 63 Assume that we specify the forward rate dynamics under a martingale measure $\mathbb{P}$ by

$$
d f(t, T, c)=\alpha_{\eta(t)}(t, T, c) d t+\sigma_{\eta(t)}(t, T, c) d W_{t}+\int_{\mathbb{R}^{r}} \delta_{\eta(t)}(t, x, T)\left(\mu_{\eta(t)}^{c}-v_{\eta(t)}^{c}\right)(d t, d x)
$$

Then the following relation holds

$$
\begin{aligned}
\alpha_{\eta(t)}(t, T, c)= & -\sigma_{\eta(t)}(t, T, c)^{\top} b_{\eta(t)}(t, T, c)+s(t, t, c) \\
& +\int_{\mathbb{R}^{d}}\left(e^{h(t, x, T)}-1-h(t, x, T)\right) \mu_{\eta(t)}^{c}(d t, d x)
\end{aligned}
$$

Proof. Since we are working under a martingale measure $\mathbb{P}$ we have by Proposition 60 that

$$
\tilde{a}_{\eta(t)}\left(t, T, C_{t-}\right)-l \cdot \lambda_{c_{t_{-}, 1}}+\int_{\mathbb{R}^{d}}\left(e^{h(t, x, T)}-1-h(t, x, T)\right) \mu_{\eta(t)}^{C_{t_{-}}}(d t, d x)=0
$$

and differentiating this equation with respect $T$ gives us the equation (2.37). 
Proposition 64 The corporate-bond price dynamics under a martingale measure $\mathbb{P}$, and under the fractional recovery of market value hypothesis, will follow the stochastic differential equation

$$
\frac{d B\left(t, T_{i}, C_{t}\right)}{B\left(t_{-}, T_{i}, C_{t_{-}}\right)}=r_{t} d t+b_{j}\left(t, T_{i}, C_{t_{-}}\right) d W_{t}+\int_{\mathbb{R}^{d}} h\left(t, x, T_{i}\right) 1_{\{|x| \leq 1\}} \tilde{\mu}_{j}^{c}(d t, d x)-l \cdot d M_{1}(t)
$$

where $\tilde{\mu}_{j}^{c}$ is the $\mathbb{P}$-compensated Lévy measure, $l=1-q \in(0,1)$ is the portion of the market value that the investor will lose in the default case.

Proof. Similarly to (2.18) we have that

$$
B\left(t, T_{i}, C_{t}\right)=B_{t} Z\left(t, T_{i}, C_{t}\right) .
$$

Then, under the risk-neutral measure,

$$
\begin{aligned}
d B\left(t, T_{j}, C_{t}\right)= & Z\left(t, T_{i}, C_{t}\right) d B_{t}+B_{t} d Z\left(t, T_{i}, C_{t}\right) \\
= & B\left(t_{-}, T_{i}, C_{t_{-}}\right)\left[r_{t} d t+b_{j}\left(t, T_{i}, C_{t_{-}}\right) d W_{t}\right. \\
& \left.+\int_{\mathbb{R}^{d}} h\left(t, x, T_{i}\right) 1_{\{|x| \leq 1\}} \tilde{\mu}_{j}^{c}(d t, d x)-l \cdot d M_{1}(t)\right]
\end{aligned}
$$

\subsubsection{Absence of arbitrage in a corporate-bond market with fractional recov- ery of treasury}

In this second subsection, we basically reproduce the results given in the last subsection, but under fractional recovery of treasury framework.

Proposition 65 The initial probability measure $\mathbb{P}$ itself belongs to $\mathcal{Q}_{\mathcal{F}}$ if and only if the following two conditions hold, for every $T_{i} \in J$ :

$$
\sum_{j \leq \eta(t)} \int_{T_{j}}^{t \wedge T_{j+1}} \int_{\mathbb{R}^{d}}\left(e^{h\left(t, x, T_{i}\right)}-1-h\left(t, x, T_{i}\right)\right) \mu_{j}^{C_{t}}(d t, d x)<\infty
$$

and

$$
\tilde{a}_{j}\left(t, T_{i}, C_{t-}\right)-\left(1-\frac{q}{S\left(t, T_{i}, c\right)}\right) \cdot \lambda_{c_{t_{-}, 1}}+\int_{\mathbb{R}^{d}}\left(e^{h\left(t, x, T_{i}\right)}-1-h\left(t, x, T_{i}\right)\right) \mu_{j}^{C_{t_{-}}}(d t, d x)=0
$$

for any $t \in\left[0, T_{i}\right]$, and any $C_{t_{-}} \in \mathcal{K} \backslash\{1\}$ where ${ }^{11}$

$$
\tilde{a}_{j}\left(t, T_{i}, C_{t-}\right):=a_{j}\left(t, T_{i}, C_{t-}\right)+s\left(t, t, C_{t-}\right)+\frac{1}{2}\left|b_{j}\left(t, T_{i}, C_{t-}\right)\right|^{2}
$$

and

$$
S\left(t, T_{i}, c\right)=\exp \left\{-\int_{t}^{T_{j}} s(t, s, c) d s\right\}
$$

Proof. Basically the proof is the same as for Proposition 60.

${ }^{11}$ Notice that if $C_{\tau_{k}}=0$ it means default-free asset and $s_{t}^{0}\left(T_{i}\right)=0$. It is the risk-free bond. 
Remark 66 Notice how in this case we obtain the following expression for the default intensity, under risk-neutral measure

$$
\lambda_{c_{t_{-}, 1}}=\frac{S\left(t, T_{i}, c\right)}{S\left(t, T_{i}, c\right)-q}\left[\tilde{a}_{j}\left(t, T_{i}, C_{t-}\right)+\int_{\mathbb{R}^{d}}\left(e^{h\left(t, x, T_{i}\right)}-1-h\left(t, x, T_{i}\right)\right) \mu_{j}^{C_{t}}(d t, d x)\right]
$$

Proposition 67 The corporate-bond price dynamics under a martingale measure $\mathbb{P}$, and under the fractional recovery of treasury hypothesis, will follow

$$
\begin{aligned}
\frac{d B\left(t, T_{i}, C_{t}\right)}{B\left(t_{-}, T_{i}, C_{t_{-}}\right)}= & r_{t} d t-\left(1-\frac{q}{S\left(t, T_{i}, c\right)}\right) \cdot d M_{1}(t) \\
& +b_{j}\left(t, T_{i}, C_{t_{-}}\right) d W_{t}+\int_{\mathbb{R}^{d}} h\left(t, x, T_{i}\right) 1_{\{|x| \leq 1\}} \tilde{\mu}_{j}^{c}(d t, d x)
\end{aligned}
$$

where $r_{t}$ is the usual short-rate, $M_{1}(t)$ is the martingale from the Doob-Meyer decomposition of the default indicator and $\tilde{\mu}_{j}^{c}$ is the $\mathbb{P}$-compensated Lévy measure.

Proof. As in Proposition 64.

\subsubsection{Absence of arbitrage in a corporate-bond market with fractional recov- ery of par value}

And finally, in this third subsection, we give the results under the hypothesis that in the case of a default the investor will recover a fractional part of par value .

Proposition 68 The initial probability measure $\mathbb{P}$ itself belongs to $\mathcal{Q}_{\mathcal{F}}$ if and only if the following two conditions hold, for every $T_{i} \in J$ :

$$
\sum_{j \leq \eta(t)} \int_{T_{j}}^{t \wedge T_{j+1}} \int_{\mathbb{R}^{d}}\left(e^{h\left(t, x, T_{i}\right)}-1-h\left(t, x, T_{i}\right)\right) v_{j}^{C_{t}}(d t, d x)<\infty
$$

and

$$
\tilde{a}_{j}\left(t, T_{i}, C_{t-}\right)-\left(1-\frac{q}{Z\left(t, T_{i}, C_{t_{-}}\right)}\right) \cdot \lambda_{c_{t_{-}, 1}}+\int_{\mathbb{R}^{d}}\left(e^{h\left(t, x, T_{i}\right)}-1-h\left(t, x, T_{i}\right)\right) v_{j}^{C_{t_{-}}}(d t, d x)=0
$$

for any $t \in\left[0, T_{i}\right]$, and any $C_{t_{-}} \in \mathcal{K} \backslash\{1\}$ where ${ }^{12}$

$$
\tilde{a}_{j}\left(t, T_{i}, C_{t-}\right):=a_{j}\left(t, T_{i}, C_{t-}\right)+s\left(t, t, C_{t-}\right)+\frac{1}{2}\left|b_{j}\left(t, T_{i}, C_{t-}\right)\right|^{2}
$$

Proof. Basically the proof is the same as for Proposition 60.

Remark 69 Notice how in this case we obtain the following expression for the default intensity, under risk-neutral measure

$$
\lambda_{c_{t_{-}, 1}}=\frac{Z\left(t, T_{i}, c\right)}{Z\left(t, T_{i}, c\right)-q}\left[\tilde{a}_{j}\left(t, T_{i}, C_{t-}\right)+\int_{\mathbb{R}^{d}}\left(e^{h\left(t, x, T_{i}\right)}-1-h\left(t, x, T_{i}\right)\right) v_{j}^{C_{t_{-}}}(d t, d x)\right]
$$

\footnotetext{
${ }^{12}$ Notice that $C_{t}=0$ means default-free bond and consequently the spread $s_{t}^{0}\left(T_{i}\right)=0$.
} 
Proposition 70 The corporate-bond price dynamics under a martingale measure $\mathbb{P}$, and under the fractional recovery of par value hypothesis, will follow

$$
\begin{aligned}
\frac{d B\left(t, T_{i}, C_{t}\right)}{B\left(t_{-}, T_{i}, C_{t_{-}}\right)}= & r_{t} d t-\left(1-\frac{q}{Z\left(t, T_{i}, c\right)}\right) \cdot d M_{1}(t) \\
& +b_{j}\left(t, T_{i}, C_{t_{-}}\right) d W_{t}+\int_{\mathbb{R}^{d}} h\left(t, x, T_{i}\right) 1_{\{|x| \leq 1\}} \tilde{\mu}_{j}^{c}(d t, d x)
\end{aligned}
$$

where $r_{t}$ is the usual short-rate, $M_{1}(t)$ is the martingale from the Doob-Meyer decomposition of the default indicator and $\tilde{\mu}_{j}^{c}$ is the $\mathbb{P}$-compensated Lévy measure.

Proof. As in Proposition 64. 


\subsection{Corporate-Bond valuation in a Reaction-Additive system}

In this section, we turn our attention to the Reaction-Additive system which can be defined as a system of stochastic differential equations (SDEs). The first of these SDEs is based on the bond price process $B$ as a tradeable risky asset which is modelled using a LIBOR additive process. On the other hand, the second SDE models the non-tradeable factor of uncertainty (credit migration) which is represented by a finite-state process $C$ driven by a multivariate point process.

The present section is mainly devoted to show how due to this system of stochastic equations we can obtain a realistic sample-path for corporate bond prices, driven by the composition of these two processes. In order to achieve this goal, we proceed in three different steps:

- First, we introduce some ideas and references where the reader can find the proof of existence and uniqueness of solutions for such SDEs systems.

- In the second subsection, we face the question of markovianity and uniqueness of the sample paths generated by the composition of these two processes.

- Finally, in the third subsection, we prove that these sample paths are 'càdlàg' or equivalently, they 'live' in a Skorohod space.

In order to clarify the framework, let us briefly summarize some assumptions and results that we have developed in the previous sections. Recall that $B\left(t, T_{i}, C_{t}\right)$ is the price of a zero-coupon corporate bond, valued in $t \in\left[0, T_{i}\right]$ for any fixed maturity $T_{i} \leq T^{\star}$, with credit rating $C_{t}$. Additionally we assume that the corporate bond is modelled with a fractional recovery of market value in case of default (see (2.26)). Notice that $B\left(t, T_{i}, C_{t}\right)$ is a strictly positive and $\mathcal{F}$-adapted process, defined on a 'sufficiently rich, ${ }^{13}$ stochastic basis $(\Omega, \mathbb{F}, \mathbb{P})$ endowed with the filtration $\mathbb{F}=\left(\mathcal{F}_{t}\right)_{t \in\left[0, T^{*}\right]}$ generated by a $d$-dimensional LIBOR additive process $G$ and the credit migration process $C$, or in other words $\mathcal{F}_{t}=\sigma\left\{G_{s}, C_{s} ; 0 \leq s \leq t\right\}$.

Let us fix the following elements: first, fix $m \in \mathbb{N}_{+}$such that $\mathcal{K}=\left\{0, \frac{1}{m}, \ldots, 1-\frac{1}{m}, 1\right\}$, second fix the time horizon $T_{i} \in\left[0, T^{\star}\right]$, as the maturity of the corporate bond; and finally, a domain, that in our case will be $\mathbb{R}^{d}$. Given any starting point $\left(t, B_{t}, C_{t}\right) \in\left[0, T_{i}\right] \times \mathbb{R}^{d} \times \mathcal{K}$ we have the following system of SDEs, where $(B, C)$ is a solution, under risk-neutral probability, with values in $\mathbb{R}^{d} \times \mathcal{K}$ :

$$
\left\{\begin{array}{l}
\frac{d B\left(t, T_{i}, C_{t}\right)}{B\left(t_{-}, T_{i}, C_{t_{-}}\right)}=\left(r_{t}+l \cdot \lambda_{C_{t-}, 1}\right) d t+b_{\eta_{(t)}}\left(t, T_{i}, C_{t_{-}}\right) d W_{t}+\int_{\mathbb{R}^{d}} h\left(t, x, T_{i}\right) 1_{\{|x| \leq 1\}} \tilde{\mu}_{\eta_{(t)}}^{C_{t-}}(d t, d x) \\
d C_{t}=\sum_{a, b \in K}(b-a) 1_{\left\{C_{t_{-}}=a\right\}}\left(C_{t_{-}}\right) d N_{a b}(t)
\end{array}\right.
$$

where $r_{t}=f(t, t, 0)$ is the usual risk-free 'short-rate', $l$ is the rate of losses in case of a default, such that $l \in[0,1], b_{\eta_{(t)}}$ is the coefficient of diffusion such that $b_{\eta_{(t)}}:\left[0, T_{i}\right] \times \mathcal{K} \rightarrow \mathbb{R}^{d}$ is $C^{1}$ with respect to $(t, B) \in\left[0, T_{i}\right] \times \mathbb{R}^{d}$ for any $t \leq T_{i}$ with $t, T_{i} \in\left[0, T^{\star}\right]$.

Notice that we have three sources of randomness,

- first, $W=\left(W^{i}\right)_{i=1 \ldots d}$ is the usual $\mathbb{R}^{d}$-valued $(\mathbb{P}, \mathcal{G})$-Brownian motion;

- second, $\tilde{\mu}_{\eta_{(t)}}^{C_{t-}}$ is the compensated random measure, for a given credit rate $C_{t-}$, that satisfy the usual integrability conditions for any $t \leq T_{i}$ with $t, T_{i} \in\left[0, T^{\star}\right]$

${ }^{13}$ See definitions in section 1.1.2 and assumptions in section 2.1.3 
- and finally, $N=\left(N_{a b}\right)_{a, b \in \mathcal{K}}$ is a $\mathcal{F}$-adapted multivariate point process such that $\left(N_{a b}(t)\right)$ has $(\mathbb{P}, \mathcal{G})$-intensity $\lambda^{a b}(t, B)$ for $a, b \in \mathcal{K}=\left\{0, \frac{1}{m}, \ldots, 1-\frac{1}{m}, 1\right\}$.

This model is a non-standard SDE system because of its dependence structure. The coefficients in the SDE (2.47) for corporate bond dynamic contain the credit risk rating and, on the other hand, the intensities in the multivariate point process $N$ that drive the credit migration process, depend in turn on the credit rate of the corporate bond.

\subsubsection{Classical and Viscosity solutions for reaction-additive systems under local regularity}

In this section we mention different methodologies to derive existence and uniqueness results for classical and viscosity solutions of interacting systems of partial integro-differential equations (PIDEs). Such systems will be called reaction-additive equations and play a key role in subsequent sections. These methodologies and results have been studied previously by different authors, such that Bensoussan and Lions (1982), Crandall and Lions (1983), Barles, Buckdahn and Pardoux (1997), or Pardoux, Pradeilles and Rao (1997). We mention here, very briefly, some of the main results, in order to show the existence of solutions to the reaction-additive system.

We consider the system of integral-partial differential equations (PIDEs) of parabolic type for $c \in \mathcal{K} \backslash\{1\}, j=0,1, \ldots, n$ and boundary conditions at terminal time $T$. Denote by USC (respectively LSC) the class of upper semicontinuous (respectively, lower semicontinuous) functions $u:(0, T] \times \mathcal{K} \times \mathbb{R}^{d} \rightarrow \mathbb{R}$ and, on the other hand, let us define by $C_{p}^{+}\left((0, T] \times \mathcal{K} \times \mathbb{R}^{d} ; \mathbb{R}\right)$ the set of measurable functions on $[0, T] \times \mathcal{K} \times \mathbb{R}^{d}$ with polynomial growth of the degree $p$ at $+\infty$, Lipschitz and bounded on $[0, T] \times \mathcal{K} \times \mathbb{R}^{-}$ such that

$$
\varphi \in C_{p}^{+}((0, T] \times \mathcal{K} \times \mathbb{R}) \Longleftrightarrow \exists K, p>0,|\varphi(t, x)| \leq K\left(1+|x|^{p} 1_{\{x>0\}}\right)
$$

Consider the following system of backward integral-partial differential equation of parabolic type (initial-boundary value problem) on $(0, T] \times \mathcal{K} \times \mathbb{R}$ for all $j=0,1, \ldots, n$

$$
\left\{\begin{array}{l}
-\frac{\partial}{\partial t} u_{j}(t, x, c)-\mathcal{L} u_{j}(t, x, c)-f_{j}\left(t, x, c, u_{j},\left(\nabla u_{j} b_{j}^{c}\right)(t, x, c), \mathcal{B} u_{j}(t, x, c)\right)=0 \\
u_{j}(T, x, c)=g_{j}(x, c)
\end{array}\right.
$$

where the second-order integral-differential operator $\mathcal{L}$ for any $c \in \mathcal{K} \backslash\{1\}$ on sufficiently smooth functions has the form

$$
\mathcal{L}=\mathcal{A}+\mathcal{J}
$$

with

$$
\begin{aligned}
\mathcal{A} u_{\eta(t)}(t, x, c)= & \sum_{i=1}^{d} a_{\eta(t)}(t, T, c) \frac{\partial u_{\eta(t)}}{\partial x^{i}}(t, x, c)+\frac{1}{2} \sum_{i, j=1}^{d} b_{\eta(t)}(t, T, c) \frac{\partial^{2} u_{\eta(t)}^{c}}{\partial x^{i} \partial x^{j}}(t, x, c) \\
& +\sum_{c \in \mathcal{K}} \lambda_{b, c}(t)\left[u_{\eta(t)}(x, t, c)-u_{\eta(t)}(x, t, b)\right] \\
\mathcal{J} u_{\eta(t)}(t, x, c)= & \int_{\mathbb{R}^{d}}\left[u_{\eta(t)}(x+y, t, c)-u_{\eta(t)}(x, t, c)-y 1_{\{|y|<1\}}\right] \mu_{\eta(t)}^{c}(d y)
\end{aligned}
$$

and $\mathcal{B}$ is an integral operator defined as

$$
\mathcal{B} u_{\eta(t)}(x, c)=\int_{\mathbb{R}^{d}}\left[u_{\eta(t)}(x+y, t, c)-u_{\eta(t)}(x, t, c)\right] \mu_{\eta(t)}^{c}(d y)
$$


Existence and uniqueness of classical solutions for the PIDEs considered above in Sobolev-Hölder spaces have been studied in Bensoussan and Lions (1982) and Garroni and Menaldi (2002) in the case where the diffusion component is not degenerated (basically with $\sigma_{\eta(t)}(t, T, c)>0$ for every $t \in[0, T]$ and $c \in \mathcal{K} \backslash\{1\})$.

However, as we have already mentioned in Chapter 1, many of the examples of the LIBOR additive process can be pure-jump processes (with $\sigma_{\eta(t)}(t, T, c)>0$ for every $t \in[0, T]$ and $c \in \mathcal{K} \backslash\{1\}$ ) for which such results are not available. A notion of solution that yields existence and uniqueness for such equations without requiring nondegeneracy of coefficients or a prior knowledge of smoothness of solutions is the notion of viscosity solution introduced by Crandall and Lions (1983) for PDEs, and extended for integro-differential equations of the type considered here in Alvarez and Tourin (1996), Barles, Buckdahn and Pardoux (1997), or Pardoux, Pradeilles and Rao (1997).

For such a system, we introduce the notion of a viscosity solution

Definition 71 We say that $u \in C_{p}^{1,2,+}\left((0, T] \times \mathcal{K} \times \mathbb{R}^{d} ; \mathbb{R}\right)$ is

(i) a viscosity subsolution of (2.48) if

$$
u_{j}(T, x, c) \leq g_{j}(x, c) \text { with } x \in \mathbb{R}^{d}
$$

and if for all $c \in \mathcal{K}$, all $j=0, \ldots, n$, and $\varphi_{j} \in C_{p}^{1,2,+}\left((0, T] \times \mathcal{K} \times \mathbb{R}^{d} ; \mathbb{R}\right)$, such that $(t, x) \in[0, T] \times \mathbb{R}^{d}$ is a global minimum point of $u_{j}-\varphi_{j}$, we have

$$
-\frac{\partial}{\partial t} \varphi_{j}(t, x, c)-\mathcal{A} \varphi_{j}(t, x, c)-\mathcal{J}\left(u_{j}, \varphi_{j}\right)(t, x, c)-f_{j}\left(t, x, u_{j}(t, x, c),\left(\nabla \varphi_{j} b_{j}^{c}\right)(t, x, c), \mathcal{B}_{c} \varphi_{j}(t, x, c)\right) \geq 0
$$

(ii) a viscosity supersolution of (2.48) if

$$
u_{j}(T, x, c) \geq g_{j}(x, c) \text { with } x \in \mathbb{R}^{d}
$$

and if for all $c \in \mathcal{K}$, all $j=0, \ldots, n$, and $\varphi_{j} \in C_{p}^{1,2,+}\left((0, T] \times \mathcal{K} \times \mathbb{R}^{d} ; \mathbb{R}\right)$, such that $(t, x) \in[0, T] \times \mathbb{R}^{d}$ is a global maximum point of $u_{j}-\varphi_{j}$,

$-\frac{\partial}{\partial t} \varphi_{j}(t, x, c)-\mathcal{A} \varphi_{j}(t, x, c)-\mathcal{J}\left(u_{j}, \varphi_{j}\right)(t, x, c)-f_{j}\left(t, x, u_{j}(t, x, c),\left(\nabla \varphi_{j} b_{j}^{c}\right)(t, x, c), \mathcal{B}_{c} \varphi_{j}(t, x, c)\right) \leq 0$

(iii) a viscosity solution of (2.48) if it is both a sub and a supersolution of (2.48)

Note that existence and uniqueness of viscosity solutions for such parabolic integro-differential equations in $\mathbb{R}^{d}$ are discussed in Alvarez and Tourin (1996) in the case where $v$ is a finite measure, and in Barles, Buckdahn and Pardoux (1997) or Pham (1998).

Theorem 72 Under the conditions that $u$ belongs to the set of measurable functions on $[0, T] \times \mathcal{K} \times \mathbb{R}^{d}$ with polynomial growth of the degree $p$ at $+\infty$, Lipschitz and bounded on $[0, T] \times \mathcal{K} \times \mathbb{R}^{-}$, then the function $u$ is a viscosity solution of the system of backward PIDEs (2.48).

Proof. cf. Barles, Buckdahn and Pardoux (1997) Theorem 3.4. or Pardoux, Pradeilles and Rao (1997) Theorem 4.1. 


\subsubsection{Markov property and uniqueness}

A standard way to show the Markov property is to prove uniqueness of a corresponding (timeinhomogeneous) martingale problem. We expose here a direct argument in the mentioned way.

Consider the mentioned reaction-additive system of SDE under risk neutral probability

$$
\left\{\begin{array}{l}
\frac{d B\left(t, T_{i}, C_{t}\right)}{B\left(t_{-}, T_{i}, C_{t_{-}}\right)}=\left(r_{t}+l \cdot \lambda_{C_{t_{-},}}\right) d t+b_{\eta_{(t)}}\left(t, T_{i}, C_{t_{-}}\right) d W_{t}+\int_{\mathbb{R}^{d}} h\left(t, x, T_{i}\right) 1_{\{|x| \leq 1\}} \tilde{\mu}_{\eta_{(t)}}^{C_{t-}}(d t, d x) \\
d C_{t}=\sum_{a, b \in K}(b-a) 1_{\left\{C_{t_{-}}=a\right\}}\left(C_{t_{-}}\right) d N_{a b}(t)
\end{array}\right.
$$

Let $h\left(B_{t}, C_{t}\right)$ be a Borel-measurable function, where $B_{t}$ means $B\left(t, T_{i}, C_{t}\right)$. Define the function $u(t, B, C) \in C_{b}(\mathbb{R} ; \mathbb{R} \times \mathcal{K})$, for any $t \in\left[0, T_{i}\right]$, that satisfies the following PIDE system

$$
\begin{aligned}
0= & \frac{\partial u_{\eta_{(t)}}}{\partial t}\left(t, B_{t}, C_{t}\right) \\
& +\left(r_{t}+l \cdot \lambda_{C_{t_{-},}}\right) B_{t_{-}} \frac{\partial u_{\eta_{(t)}}}{\partial B}\left(t, B_{t}, C_{t}\right) \\
& +\frac{b_{\eta_{(t)}}\left(t, T, C_{t_{-}}\right)^{2}}{2} B_{t_{-}} \frac{\partial^{2} u_{\eta_{(t)}}}{\partial B^{2}}\left(t, B_{t}, C_{t}\right) \\
& +\int_{\mathbb{R}^{d}}\left[u_{\eta_{(t)}}\left(t, B_{t_{-}} e^{x}, C_{t_{-}}\right)-u_{\eta_{(t)}}\left(t, B_{t_{-}}, C_{t_{-}}\right)-B_{t_{-}}\left(e^{x}-1\right)\right] v_{\eta_{(t)}}^{C_{t_{-}}}(d x) \\
& +\frac{\partial u_{\eta_{(t)}}}{\partial C}\left(t, B_{t}, C_{t}\right) \sum_{a, b \in K}\left[u_{\eta_{(t)}}\left(t, B_{t}, a\right)-u_{\eta_{(t)}}\left(t, B_{t}, b\right)\right] 1_{\left\{C_{t_{-}}=b\right\}}\left(C_{t_{-}}\right) \lambda_{t}^{a b}
\end{aligned}
$$

for $a, b \in \mathcal{K}, x \in \mathbb{R}^{d}$ and terminal condition

$$
u_{\eta_{(T)}}(T, B, C)=h\left(B_{T}, C_{T}\right)
$$

Proposition 73 For $u_{\eta_{(t)}}$ given as above, the process $u_{\eta_{(t)}}\left(t, B_{t}, C_{t}\right)$ with $t \in\left[0, T_{i}\right]$ is a martingale.

Proof. Applying Itô formula to $u_{\eta_{(t)}}\left(t, B_{t}, C_{t}\right)$ yields

$$
\begin{aligned}
d u_{\eta_{(t)}}\left(t, B_{t}, C_{t}\right)= & \frac{\partial u_{\eta_{(t)}}}{\partial B_{t}}\left(t, B_{t}, C_{t}\right) d B_{t} \\
& +\left(\frac{\partial u_{\eta_{(t)}}}{\partial t}\left(t, B_{t}, C_{t}\right)+\frac{1}{2} b_{j}(t, T, c)^{2} \frac{\partial^{2} u}{\partial B_{t}^{2}}\left(t, B_{t}, C_{t}\right)\right) d t \\
& +\int_{\mathbb{R}^{d}}\left[u_{\eta_{(t)}}\left(t, B_{t_{-}} e^{x}, C_{t_{-}}\right)-u_{\eta_{(t)}}\left(t, B_{t_{-}}, C_{t_{-}}\right)-B_{t_{-}}\left(e^{x}-1\right)\right] v_{\eta_{(t)}}^{C_{t_{-}}}(d x) \\
& +\sum_{a, b \in K}\left[u_{\eta_{(t)}}\left(t, B_{t_{-}}, a\right)-u_{\eta_{(t)}}\left(t, B_{t_{-}}, b\right)\right] 1_{\left\{C_{t_{-}}=b\right\}}\left(C_{t_{-}}\right) d N_{t}^{a b}
\end{aligned}
$$

where substituting the Doob-Meyer decomposition, $d N_{t}^{a b}=d M_{t}^{a b}+\lambda_{t}^{a b} d t$ and using PIDE (2.48) 
yields that the drift term vanishes on $[0, T]$. From this we obtain

$$
\begin{aligned}
d u_{\eta_{(t)}}\left(t, B_{t}, C_{t}\right)= & B_{t_{-}} \frac{\partial u_{\eta_{(t)}}}{\partial B_{t}}\left(t, B_{t}, C_{t}\right) b_{\eta_{(t)}}\left(t, T_{i}, C_{t_{-}}\right) d W_{t} \\
& +B_{t_{-}} \frac{\partial u_{\eta_{(t)}}}{\partial B_{t}}\left(t, B_{t}, C_{t}\right) \int_{\mathbb{R}^{d}} h\left(t, x, T_{i}\right) 1_{\{|x| \leq 1\}} \tilde{\mu}_{j}^{c}(d t, d x) \\
& +\sum_{a, b \in K}\left[u_{\eta_{(t)}}\left(t, B_{t_{-}}, a\right)-u_{\eta_{(t)}}\left(t, B_{t_{-}}, b\right)\right] 1_{\left\{C_{t_{-}}=b\right\}}\left(C_{t_{-}}\right) d M_{t}^{a b}
\end{aligned}
$$

Therefore the process $u_{j}\left(t, B_{t}, C_{t}\right), t \in\left[0, T_{j}\right]$ is a martingale.

Notice that the last theorem is equivalent to the following fact: for any continuous function $f\left(t, G_{t}^{C}\right)$ on $[0, T] \times\left[\mathbb{R}^{d} \times \mathcal{K}\right]$ with compact support that is of class $\mathcal{C}^{2}$ in $g$, the process

$$
f\left(t, G_{t}^{C}\right)-f\left(0, G_{0}^{C}\right)-\int_{0}^{t} \mathcal{L}_{s} f\left(s, G_{s}^{C}\right) d s
$$

is a martingale, with the operator $\mathcal{L}_{s}$ being given by

$$
\begin{aligned}
\mathcal{L}_{s} f\left(s, G_{s}^{C}\right)= & \sum_{i=1}^{d} \alpha_{\eta_{(s)}}^{i}(t, C) \frac{\partial f}{\partial G^{i, C}}\left(t, G_{t}^{C}\right)+\frac{1}{2} \sum_{i, j=1}^{d} \beta_{\eta_{(s)}}^{i, j}(t, C) \frac{\partial^{2} f}{\partial G^{i, C} \partial G^{j, C}}\left(t, G_{t}^{C}\right) \\
& +\sum_{b, c \in \mathcal{K}} \lambda_{b, c}(t)\left[f\left(t, G_{t}^{c}\right)-f\left(t, G_{t}^{b}\right)\right]+\int_{\mathbb{R}^{d}}\left[f\left(t, G_{t}^{c}+g\right)-f\left(t, G_{t}^{c}\right)-g 1_{\{|g|<1\}}\right] \mu_{\eta(s)}(d g)
\end{aligned}
$$

Proposition $74 G_{t}^{C}$ with $t \in\left[0, T_{j}\right]$ is a (time-inhomogeneous) Markov process with respect to $\mathbb{P}$ and $\mathcal{F}$. Its distribution is uniquely determined by the SDE system.

Proof. For any $h \in C_{b}\left(\mathbb{R}^{d} \times \mathcal{K}, \mathbb{R}\right)$ there is a unique viscosity solution $u$ to the PIDE (2.48). By Barles, Buckdahn and Pardoux (1997) Theorem 3.4 or Pardoux, Pradeilles and Rao (1997) Theorem 4.1 we have

$$
\mathbb{E}\left[h\left(G_{T}, C_{T}\right) \mid \mathcal{F}_{t}\right]=\mathbb{E}\left[u\left(T, G_{T}, C_{T}\right) \mid \mathcal{F}_{t}\right]=u\left(t, G_{t}, C_{t}\right)
$$

for $0 \leq t \leq T \leq T^{\star}$, and this establishes the Markov property of $(G, C)$ (by Theorem 38.ii).

To show uniqueness of the finite-dimensional distributions by induction, let $h_{1}, \ldots, h_{m-1}, h_{m}$ be arbitrary continuous bounded functions. For any times $t_{0} \leq \ldots \leq t_{m-1} \leq t_{m}$ conditioning on $\mathcal{F}_{m-1}$

$$
\mathbb{E}\left[\prod_{j=0}^{m} h_{j}\left(G_{t_{j}}, C_{t_{j}}\right) \mid \mathcal{F}_{m-1}\right]=\mathbb{E}\left[\left(\prod_{j=0}^{m-1} h_{j}\left(G_{t_{j}}, C_{t_{j}}\right) \mid \mathcal{F}_{m-1}\right) u_{\eta_{\left(t_{m-1}\right)}}\left(G_{t_{m-1}}, C_{t_{m-1}}\right)\right]
$$

where $u_{\eta_{\left(t_{j}\right)}}$ denotes the solution to the PIDE (2.48) in $t_{j}$. Since the right-hand side of the last equation is determined by the $\mathrm{n}$-dimensional distributions, the claims follow.

\subsubsection{Sample-paths in the Skorohod space $D_{\left[0, T^{\star}\right]}\left(\mathbb{R}^{d}, \mathcal{K}\right)$}

Let us recall that the main "engine" that moves our system of stochastic differential equation is a LIBOR additive process with credit transitions $G^{C}$ such that $G^{C}:(\omega, t) \rightarrow\left(G_{t}, C_{t}\right) \equiv\left(G_{t}^{C}\right)_{C \in \mathcal{K}}$ for every $t \in\left[0, T^{\star}\right]$ and $C \in \mathcal{K}^{(m)}=\left\{0, \frac{1}{m}, \ldots, 1-\frac{1}{m}, 1\right\}$ with a fixed $m \in \mathbb{N}_{+}$. The main goal of this subsection 
is to specify the necessary and sufficient conditions for $G^{C} \in D_{\left[0, T^{\star}\right]}\left(\mathbb{R}^{d}, \mathcal{K}\right)$, or in other words, $G^{C}$ has sample paths in the Skorohod space or the space of real functions $G^{C}(t)$ on $\left[0, T^{\star}\right] \times \mathbb{R}^{d} \times \mathcal{K}^{(m)}$ that are 'càdlàg' (right-continuous with left-limits).

Notice that if we consider $C$ fixed with value $c \in \mathcal{K}$ with $\mathcal{K} \in[0,1] \cap \mathbb{Q}$, then $G^{c} \in D_{\left[0, T^{*}\right]}\left(\mathbb{R}^{d}, \mathcal{K}\right)$ by direct application of Theorem 11.1 in Sato (1999), or roughly speaking, if $G^{c}$ is a stochastically continuous and Markov process then it has a version in the Skorohod space $D_{\left[0, T^{\star}\right]}\left(\mathbb{R}^{d}, \mathcal{K}\right)$. Properties of such processes and spaces are very well-known and perfectly reflected in Lipster and Shiryaev (1989) or Jacod and Shiryaev (1999). Our aim in this subsection is to extend these results when $G^{C}$ is a semimartingale that depends directly on the process $C$.

There exist different ways to prove that $G^{C}$ has sample-functions in the Skorohod space $D_{\left[0, T^{\star}\right]}\left(\mathbb{R}^{d}, \mathcal{K}\right)$. In our case, we will follow the most generic manner, closely related to Billingsley (1999), Carmona, Kesten and Walsh (1986), Jacod and Shiryaev (1987), Liptser and Shiryaev (1989), and Vostrikova (1988).

Let us begin with the following definitions:

- Let $\Pi_{\delta}^{(n)}$ be the partition $\left\{0=t_{0}<t_{1}<\ldots<t_{n} \leq T^{\star} \leq 1\right\}$ of the time interval $\left[0, T^{\star}\right]$, where $n \in$ $\mathbb{N}_{+}$, satisfying the condition $\min _{0 \leq j \leq n-1}\left(t_{j}-t_{j-1}\right)>\delta$ on the "normalized" interval $[0,1]$

- And let $\mathcal{K}^{(m)}$ be the set of credit-ratings $\left\{0=C_{0}<C_{1}<\ldots<C_{m}=1\right\}$ where $m \in \mathbb{N}_{+}$.

Let us define the following moduli of continuity in $D_{\left[0, T^{\star}\right]}\left(\mathbb{R}^{d}, \mathcal{K}\right)$

$$
\begin{aligned}
\mathbb{L}_{\delta}^{(m)}(G) & =\inf _{\substack{\Pi_{\delta}^{n} \\
t_{j+1}-t_{j}>\delta}} \max _{0 \leq j \leq n} \sup _{s, t \in\left[t_{j}, t_{j+1}\right)} \sup _{c \in \mathcal{K}^{(m)}}\left|G_{s}^{c}-G_{t}^{c}\right| \\
\mathbb{L} \mathbb{L}_{\delta}^{(m)}(G) & =\sup _{0 \leq t \leq 1} \sup _{\substack{C^{\prime}, C \in \mathcal{K}^{(m)} \\
\left|C^{\prime}-C\right| \leq \delta}}\left|G_{t}^{C^{\prime}}-G_{t}^{C}\right|
\end{aligned}
$$

with $n, m \in \mathbb{N}_{+}, T^{\star} \in \mathbb{R}_{+}$and $\delta \in \mathbb{R}_{+}$.

Additionally, let us define an additional modulus of continuity in $D_{\left[0, T^{\star}\right]}\left(\mathbb{R}^{d}, \mathcal{K}\right)$

$$
\mathbb{L}_{\delta}^{C}(G)=\inf _{\substack{\Pi_{\delta}^{n} \\ t_{j+1}-t_{j}>\delta}} \max _{0 \leq j \leq n} \sup _{s, t \in\left[t_{j}, t_{j+1}\right)}\left|G_{s}^{C}-G_{t}^{C}\right|
$$

Theorem $75 G^{C}$ belongs to $D_{\left[0, T^{\star}\right]}\left(\mathbb{R}^{d}, \mathcal{K}\right)$ if and only, if for every $n, m \in \mathbb{N}_{+}, T^{\star} \in \mathbb{R}_{+}$the following conditions hold:

$$
\lim _{\delta \rightarrow 0} \mathbb{L}_{\delta}^{C}(G)=0
$$

and

$$
\lim _{\delta \rightarrow 0} \mathbb{L} \mathbb{L}_{\delta}^{(m)}(G)=0
$$

In order to prove this theorem, we need three additional results: The first result (Lemma 76) came directly from Billingsley (1999), and jointly with the second one (Lemma 77), both give us the basic condition or criteria to establish when $G^{C}$ belongs to $D_{\left[0, T^{\star}\right]}\left(\mathbb{R}^{d}, \mathcal{K}\right)$. The third result (Lemma 78) develops a basic tool to be used in the proof of Theorem 75 .

Lemma 76 For each $G^{C}$ in $D_{\left[0, T^{\star}\right]}\left(\mathbb{R}^{d}, \mathcal{K}\right)$ with $m \in \mathbb{N}_{+}$and $\varepsilon>0$, there exists $n \in \mathbb{N}_{+}$such that

$$
0=t_{0}<t_{1}<\ldots<t_{n} \leq T^{\star} \leq 1
$$


and

$$
\max _{0 \leq j \leq n} \sup _{s, t \in\left[t_{j}, t_{j+1}\right)} \sup _{C \in \mathcal{K}_{\delta}^{(m)}}\left|G_{s}^{C}-G_{t}^{C}\right|<\varepsilon
$$

Proof. cf. Billingsley (1999) Lemma 1 p.122.

Lemma 76 is equivalent to the following assertion.

Lemma $77 G^{C}$ belongs to $D_{\left[0, T^{\star}\right]}\left(\mathbb{R}^{d}, \mathcal{K}\right)$ if and only if for every $n, m \in \mathbb{N}_{+}, T^{\star} \in \mathbb{R}_{+}$and $\delta \in \mathbb{R}_{+}$, the following conditions hold:

$$
\lim _{\delta \rightarrow 0} \mathbb{L}_{\delta}^{(m)}(G)=0
$$

Proof. Fixed a $T^{\star} \leq 1$, we defined $D_{\left[0, T^{\star}\right]}\left(\mathbb{R}^{d}, \mathcal{K}\right)$ as the Skorohod space of functions in $\left[0, T^{\star}\right]$ with values in $\mathcal{K}^{(m)}$ which are right-continuous at any point in $\left[0, T^{\star}\right]$ and left-limits at any point in $\left[0, T^{\star}\right]$ for every $m \in \mathbb{N}_{+}$. It is clear that $G^{C}$ belongs to $D_{\left[0, T^{\star}\right]}\left(\mathbb{R}^{d}, \mathcal{K}\right)$ if it belongs to $D_{[0,1]}\left(\mathbb{R}^{d}\right)$ for any $T^{\star} \in[0,1]$ and for any $m \in \mathbb{N}_{+}$, and it is not difficult to see that Billingsley's proof of Lemma 26 for $D_{[0,1]}\left(\mathbb{R}^{d}\right)$ can be extended for $D_{\left[0, T^{\star}\right]}\left(\mathbb{R}^{d}, \mathcal{K}\right)$ changing the absolute value || of $\mathbb{R}$ with the expression $\sup _{C \in \mathcal{K}^{(m)}}||$ for any $m \in \mathbb{N}_{+}$.

Before we came to the next lemma, let us introduce the following notation: fix any $m \in \mathbb{N}_{+}$and $\bar{\delta}>0$, then $\mathcal{K}^{(m)}$ can be covered by a finite union of open-balls with radius $\bar{\delta}$. Therefore there are $C_{1}, \ldots, C_{r(\bar{\delta})}$ in $\mathcal{K}^{(m)}$ such that $B\left(C_{k}, \bar{\delta}\right)$ with $k=1, \ldots, r(\bar{\delta})$

$$
\mathcal{K}^{(m)} \subseteq B\left(C_{1}, \bar{\delta}\right) \cup \ldots \cup B\left(C_{r(\bar{\delta})}, \bar{\delta}\right)
$$

Lemma 78 For any $n, m \in \mathbb{N}_{+}, \delta>0$ and $\bar{\delta}>0$, the following conditions hold:

(1)

$$
\sup _{C \in \mathcal{K}^{(m)}} \mathbb{L}_{\delta}^{C}(G) \leq \mathbb{L}_{\delta}^{(m)}(G)
$$

(2) There is a $\delta_{0}, 0 \leq \delta_{0} \leq \delta$ such that

$$
\mathbb{L}_{\delta_{0}}^{(m)}(G) \leq 2 \max _{1 \leq k \leq r(\bar{\delta})} \mathbb{L}_{\delta}^{C_{k}}(G)+2 \mathbb{L} \mathbb{L}_{\bar{\delta}}^{(m)}(G)
$$

Proof. The inequality (1) follows from taking into account that

$$
\left|G_{s}^{C}-G_{t}^{C}\right| \leq \sup _{C \in \mathcal{K}^{(m)}}\left|G_{s}^{C}-G_{t}^{C}\right| .
$$

Therefore

$$
\inf _{\substack{\Pi_{\delta}^{(n)} \\ t_{j+1}-t_{j}>\delta}} \max _{0 \leq j \leq n} \sup _{s, t \in\left[t_{j}, t_{j+1}\right)}\left|G_{s}^{C}-G_{t}^{C}\right| \leq \inf _{\substack{\Pi_{\delta}^{(n)} \\ t_{j+1}-t_{j}>\delta}} \max _{0 \leq j \leq n} \sup _{s, t \in\left[t_{j}, t_{j+1}\right)} \sup _{C \in \mathcal{K}^{(m)}}\left|G_{s}^{C}-G_{t}^{C}\right|
$$

and this proves inequality (1).

In order to prove the inequality $(2)$, notice that for any $k=1, \ldots, r(\bar{\delta})$, we can write

$$
\left|G_{s}^{C}-G_{t}^{C}\right| \leq\left|G_{s}^{C}-G_{s}^{C_{k}}\right|+\left|G_{s}^{C_{k}}-G_{t}^{C_{k}}\right|+\left|G_{t}^{C_{k}}-G_{t}^{C}\right| .
$$


Therefore

$$
\sup _{C \in B\left(C_{k}, \bar{\delta}\right) \cap \mathcal{K}^{(m)}}\left|G_{s}^{C}-G_{t}^{C}\right| \leq 2 \mathbb{L}_{\bar{\delta}}^{(m)}(G)+\left|G_{s}^{C_{k}}-G_{t}^{C_{k}}\right|
$$

Notice that we can easily establish

$$
\sup _{C \in \mathcal{K}^{(m)}}\left|G_{s}^{C}-G_{t}^{C}\right|=\max _{1 \leq k \leq r(\bar{\delta})} \sup _{C \in B\left(C_{k}, \bar{\delta}\right) \cap \mathcal{K}^{(m)}}\left|G_{s}^{C}-G_{t}^{C}\right| \leq 2 \mathbb{L} \mathbb{L}_{\bar{\delta}}^{(m)}(G)+\max _{1 \leq k \leq r(\bar{\delta})}\left|G_{s}^{C_{k}}-G_{t}^{C_{k}}\right|
$$

and for any $\delta^{\prime}>0$ we have

$$
\begin{aligned}
\mathbb{L}_{\delta^{\prime}}^{(m)}(G) & \leq 2 \mathbb{L}_{\bar{\delta}}^{(m)}(G)+\inf _{\substack{\Pi_{\delta}^{(n)} \\
\left(t_{j+1}-t_{j}\right)>\delta}} \max _{0 \leq j \leq n} \sup _{s, t \in\left[t_{j}, t_{j+1}\right)} \max _{1 \leq k \leq r(\bar{\delta})}\left|G_{s}^{C_{k}}-G_{t}^{C_{k}}\right| \\
& \leq 2 \mathbb{L} \mathbb{L}_{\bar{\delta}}^{(m)}(G)+\inf _{\Pi_{\delta}^{(n)}} \max _{1 \leq k \leq r(\bar{\delta})} \max _{0 \leq j \leq n} \sup _{s, t \in\left[t_{j}, t_{j+1}\right)}\left|G_{s}^{C_{k}}-G_{t}^{C_{k}}\right|
\end{aligned}
$$

To achieve the inequality (2) in our Lemma 78, we just need to prove the existence of a $\delta_{0}, 0 \leq \delta_{0} \leq \delta$ such that

$$
\begin{aligned}
\inf _{\substack{\Pi_{\delta_{0}}^{(n)} \\
\left(t_{j+1}-t_{j}\right)>\delta_{0}}} \max _{1 \leq k \leq r(\bar{\delta})} \max _{0 \leq j \leq n} \sup _{s, t \in\left[t_{j}, t_{j+1}\right)}\left|G_{s}^{C_{k}}-G_{t}^{C_{k}}\right| & \leq 2 \max _{1 \leq k \leq r(\bar{\delta})} \inf _{\Pi_{\delta}^{(n)}} \max _{0 \leq j \leq n} \sup _{s, t \in\left[t_{j}, t_{j+1}\right)}\left|G_{s}^{C_{k}}-G_{t}^{C_{k}}\right| \\
& =2 \max _{1 \leq k \leq r(\bar{\delta})} \mathbb{L}_{\delta}^{C_{k}}(G)
\end{aligned}
$$

Notice that by definition of infimum, for every $k=1, \ldots, r(\bar{\delta})$ there exists a subpartition on $\left[0, T^{\star}\right]$ $\left\{t_{k, j}\right\}_{0 \leq j \leq n_{k}+1}$ such that $t_{k, j+1}-t_{k, j} \geq \delta$ for any $j=0, \ldots, n_{k}-1$ and

$$
\max _{0 \leq j \leq n_{k}} \sup _{s, t \in\left[t_{k, j}, t_{k, j+1}\right)}\left|G_{s}^{C_{k}}-G_{t}^{C_{k}}\right| \leq 2 \mathbb{L}_{\delta}^{C_{k}}(G)
$$

Define $\left\{t_{p}\right\}_{0 \leq p \leq l+1}$ as the subpartition of the interval $\left[0, T^{\star}\right]$ built with the set of different $t_{k, j}$ with $j=0, \ldots, n_{k}+1, k=1, \ldots, r(\bar{\delta})$. Finally let us define as $\delta_{0}$ the minimum distance between two points of the given partition. Therefore we have $0<\delta_{0} \leq \delta$ and

$$
\max _{0 \leq k \leq r(\bar{\delta})} \max _{0 \leq p \leq l} \sup _{s, t \in\left[t_{p}, t_{p+1}\right)}\left|G_{s}^{C_{k}}-G_{t}^{C_{k}}\right| \leq 2 \max _{1 \leq k \leq r(\bar{\delta})} \mathbb{L}_{\delta}^{C_{k}}(G)
$$

so that we finally obtain

$$
\inf _{\substack{\Pi_{\delta_{0}}^{(n)} \\ t_{j+1}-t_{j}>\delta_{0}}} \max _{1 \leq k \leq r(\bar{\delta})} \max _{0 \leq j \leq n} \sup _{\substack{1 \leq t \in\left[t_{j}, t_{j+1}\right) \\ 0}}\left|G_{s}^{C_{k}}-G_{t}^{C_{k}}\right| \leq 2 \max _{1 \leq k \leq r(\bar{\delta})} \mathbb{L}_{\delta}^{C_{k}}(G)
$$

Finally, we can prove the Theorem 75, using the last three results:

Proof. (Theorem 75) Here we attempt to show if the condition that appear in Lemma 77 is equivalent to conditions (1) and (2) in Theorem 75.

First, let us assume the conditions in Theorem 75 in the following sense: given a $\varepsilon>0$ there exists a 
$\delta_{1}>0$ with $\delta \leq \delta_{1}$ such that

$$
\mathbb{L I}_{\delta}^{(m)}(G) \leq \frac{\varepsilon}{4}
$$

and related with condition (1) in Theorem 75, there exist a $\delta_{2}>0$ with $\delta \leq \delta_{2}$ such that, for every $k=1, \ldots, r\left(\delta_{1}\right)$

$$
\mathbb{L}_{\delta}^{C_{k}}(G) \leq \frac{\varepsilon}{4}
$$

Notice that the condition (2) in Lemma 78 guarantees the existence of $\delta_{0}$ such that $0 \leq \delta_{0} \leq \delta_{2}$ and

$$
\mathbb{L}_{\delta_{0}}^{(m)}(G) \leq 2 \max _{1 \leq k \leq r(\bar{\delta})} \mathbb{L}_{\delta}^{C_{k}}(G)+2 \mathbb{L} \mathbb{L}_{\bar{\delta}}^{(m)}(G) .
$$

It is easy to see that $\mathbb{L}_{\delta}^{(m)}(G)$ is an increasing function of $\delta$, therefore we have that for every $\delta \leq \delta_{0}$

$$
\mathbb{L}_{\delta}^{(m)}(G) \leq \varepsilon
$$

This proves the condition of Lemma 77 .

Second, notice that if we assume the condition that appears in Lemma 77 and using the first assertion in Lemma 78, then the first condition in Theorem 75 is proved for every $C \in \mathcal{K}^{(m)}$. On the other hand, to establish the condition (2) in Theorem 75, let us consider a $\varepsilon>0$, and assuming the condition that appears in Lemma 78, then there exists $\Delta_{0}>0$ and a partition $0=t_{0}<t_{1}<\ldots<t_{n}=1$ on $[0,1]$ such that $t_{j}-t_{j-1}>\Delta_{0}$ for any $j=1, \ldots, n$ and

$$
\max _{0 \leq j \leq n} \sup _{s, t \in\left[t_{j}, t_{j+1}\right)} \sup _{C \in \mathcal{K}^{(m)}}\left|G_{s}^{C}-G_{t}^{C}\right| \leq \frac{\varepsilon}{3} .
$$

Notice that for any $j=1, \ldots, n$ the mapping $G_{t_{j}}: C_{t_{j}} \rightarrow G_{t_{j}}^{C}$ from $[0,1]$ to $\mathbb{R}^{d}$ is a continuous function over the compact $\mathcal{K}^{(m)}$. Hence it is uniformly continuous. Additionally for any $j=1, \ldots, n$ there is a $\delta_{j}>0$ such that for any $\delta \in\left[0, \delta_{j}\right]$

$$
\sup _{\substack{C^{\prime}, C \in \mathcal{K}^{(m)} \\\left|C^{\prime}-C\right| \leq \delta}}\left|G_{t_{j}}^{C^{\prime}}-G_{t_{j}}^{C}\right| \leq \frac{\varepsilon}{3}
$$

Let us define $\delta^{*}=\min \left(\Delta_{0}, \delta_{0}, \ldots, \delta_{n}\right)$. Then for any $t \in\left[0, T^{\star}\right]$, and choosing $t_{j}$ under the condition that $t \in\left[t_{j-1}, t_{j}\right)$, we have for any $\delta>0$

$$
\begin{aligned}
& \sup _{C^{\prime}, C \in \mathcal{K}^{(m)}}\left|G_{t}^{C^{\prime}}-G_{t}^{C}\right| \leq \sup _{C \in \mathcal{K}^{(m)}}\left|G_{t_{j}}^{C}-G_{t}^{C}\right|+\sup _{C^{\prime} \in \mathcal{K}^{(m)}}\left|G_{t_{j}}^{C^{\prime}}-G_{t}^{C^{\prime}}\right| \\
& \left|C^{\prime}-C\right| \leq \delta \\
& +\sup _{\substack{C^{\prime}, C \in \mathcal{K}^{(m)} \\
\left|C^{\prime}-C\right| \leq \delta}}\left|G_{t_{j}}^{C}-G_{t_{j}}^{C^{\prime}}\right|
\end{aligned}
$$

Using the inequalities shown before, for any $0<\delta \leq \delta^{*}$, we conclude the condition (2) of Theorem 75 from

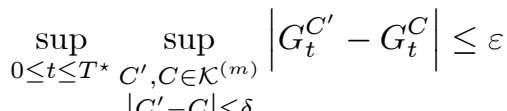

$$
\begin{aligned}
& \left|C^{\prime}-C\right| \leq \delta
\end{aligned}
$$




\subsection{Uniform Weak Convergence in the Reaction-Additive sys- tem}

In order to implement certain models and pricing rules, continuous-time models are often too complex to handle. Therefore it is convenient to both discretize time and space and show that the discretization is good in the sense that the discretized models and pricing rules converge to the continuous-time model as the discretization steps tend to zero. The fourth subject of this chapter is presented in Section 2.5 and treats this aspect of mathematical finance. We study both the approximation of a continuous-time model by a sequence of discrete-time models and the convergence of price processes for corporate bonds with credit migration.

Some authors have presented different discrete-time solutions to model defaultable or corporate bonds (Jarrow and Turnbull (1995), Jarrow, Turnbull and Lando (1997) or Schönbucher (2002) among others) as a mixture of trees, one for the risk-free interest rates, and a second one for the credit risk factor (untradeable). However, though different authors propose a useful methodology to price credit derivatives, they do not talk about the conditions of weak convergence when both factors are mixed using discrete frameworks. In fact, there is no guarantee that these compositions between random factors converge in a measure in continuous time, and all the usual tools from stochastic analysis in continuous time can be applied.

On the other hand, at least in the case of the interest-rates derivatives market, the market quotes caps/floors and swaptions (plain vanilla interest-rates derivatives) using the well-known Black-76 model. It basically implies that any alternative model written or developed to price exotic options has to guarantee that, at least, it will recover the prices of the plain-vanilla options priced by Black-76. In other words, our model has to converge in distribution to the implied distribution given by the market through the quoted volatilities. Therefore, any new interest rate model has to prove that when it goes to continuous time, it is able to recover the implied distribution in the Black-76 model, which is driven by the usual Brownian motion (continuous process). Therefore we have to prove that our LIBOR additive process (semimartingale) is able to converge weakly to the implied probability given by the market. However, our problem is more complex if we add the reactive or credit risk part to our usual stochastic differential equation.

Basically, this section is concerned with the approximation of a financial corporate bond model in incomplete markets, where the corporate bond dynamics is driven by a LIBOR additive process (tradeable) conditioned to a multivariate point process (non-tradeable).

According to the previous section, we have seen that our reaction-additive system has sample-paths in the Skorohod space. Therefore, in order to study the weak convergence of this system in such spaces, we have structured this section in the following parts:

- The first subsection is basically devoted to give a quick review of the basics related with weak convergence in the Skorohod space.

- The second one is devoted to show the conditions of relative compactness of a subset in the Skorohod space. Basically, in this subsection, we expose an equivalent result to the well-known Arzelà-Ascoli Theorem for the Skorohod space.

- In the third subsection our efforts are mainly focused to find the basic conditions in order to obtain the mentioned convergence in law, in the space $D_{\left[0, T^{\star}\right]}\left(\mathbb{R}^{d}, \mathcal{K}\right)$.

- However these conditions would be too theoretical to have any practical application in finance, and in the fourth subsection, we translate these conditions in terms of the characteristic triplets, which are the basic parameters for any derivative pricing. 


\subsubsection{Introduction to Weak Convergence}

Let us first introduce a preliminary section in order to give a brief introduction to weak convergence of stochastic processes and semimartingales. Inclusion of this material is justified not only because of the complexity of the subject but also because it is necessary to establish some basic framework, notation and theorems that will be used later.

Therefore, this preliminary section is an attempt to gather some basic and typical results to describe several main concepts and theorems that will give us the main directions of this chapter. Again, here it is not intended to give a systematic presentation of the most important results or to explain how to prove them; for this purposes one would need more pages. A more comprehensive picture of the present state of the art can be obtained from Billingsley (1968), Lipster and Shiryaev (1989) or Jacod and Shiryaev (1999).

\section{Weak Convergence, Continuous Mapping and Skorohod Embedding}

In this preliminary subsection we recall some results concerning tightness and convergence of sequences of semimartingales. As we are concerned with the weak convergence (in distribution), we suppose that for a sequence $\left(G^{n}\right)_{n \in \mathbb{N}}$ of processes, $G^{n}$ is defined on a stochastic basis $\left(\Omega^{n}, \mathbb{G}^{n}, \mathbb{P}^{n}\right)$. Additionally, a process $G$ is defined on some $(\Omega, \mathbb{G}, \mathbb{P})$, and we denote weak convergence of $G^{n}$ to $G$ i.e. $\mu^{n}=$ $\mathcal{L}\left(\left.G^{n}\right|_{\mathbb{P}^{n}}\right) \rightarrow \mu=\mathcal{L}\left(\left.G\right|_{\mathbb{P}}\right)$, by $G^{n} \rightarrow_{\mathcal{L}} G$ if there is no ambiguity about $\mathbb{P}^{n}$ and $\mathbb{P}$.

Following Jacod and Shiryaev (1999), let us consider a Polish space (E, $d$ ) (that is a complete and separable metric space) with its Borel $\sigma$-field $\mathcal{E}=\mathcal{B}_{E}$, and consider the space $\mathcal{P}(E)$ of all probability measures on $(E, \mathcal{E})$. The set $\mathcal{P}(E)$ is endowed with the weak topology which is the coarsest topology for which the mapping $\mu \rightarrow \mu(f)=\int_{E} f d \mu$ is continuous, for all bounded continuous functions $f$ on $E$. $\mathcal{P}(E)$ is itself a Polish space for this topology.

Definition 79 The sequence $\left(\mu^{n}\right)_{n \in \mathbb{N}}$ converges weakly to $\mu$ if, for every bounded continuous function $f$ on $E,\left(\mu^{n}(f)\right)_{n \in \mathbb{N}}$ converges to $\mu(f)$.

The weak convergence of random variables is defined through the weak convergence of probabilities measures: let $G$ be an $E$-valued random variable on some probability space $(\Omega, \mathbb{G}, \mathbb{P})$ The image of $\mathbb{P}$ under $G$ is denoted by $\mu=\mathbb{P}_{G}$. It is called the law or distribution of $G$.

Definition $80\left(G^{n}\right)_{n \in \mathbb{N}}$ converges in law (or in distribution) if $\left(\mu^{n}\right)_{n \in \mathbb{N}}$ converges weakly to $\mu$ in $\mathcal{P}(E)$.

This is equivalent to saying that $\mathbb{E}_{\mathbb{P}_{n}}\left[f\left(G^{n}\right)\right] \rightarrow \mathbb{E}_{\mathbb{P}}[f(G)]$ when $n \rightarrow \infty$, for all bounded continuous functions $f$ on $E$.

Notice that we can not use other standard modes of convergence on $E$ such as convergence almost surely or convergence in probability because the random variables $G^{n}$ may be defined on different probability spaces.

Now, let us recall two well-known results concerning weak convergence, namely the continuous mapping theorem and the Skorohod embedding theorem.

Theorem 81 (Continuous Mapping Theorem) Let $(E, d)$ and $\left(E^{\prime}, d^{\prime}\right)$ be two metric spaces, endowed with the Borel- $\sigma$-algebras $\mathcal{B}_{E}$ and $\mathcal{B}_{E^{\prime}}$ respectively, and let $\mu,\left(\mu^{n}\right)_{n \in \mathbb{N}}$ be probability measures on $\left(E, \mathcal{B}_{E}\right)$. 
Let furthermore $\varphi^{n}, \varphi: E \rightarrow E^{\prime}$ be a sequence of measurable functions and denote by $D$ the set of all $g \in E$ such that there exist a sequence $\left(g^{n}\right)_{n \in \mathbb{N}}$ with $g^{n} \rightarrow g$ but $\varphi^{n}\left(g^{n}\right) \nrightarrow \varphi(g)$. If $E^{\prime}$ is separable, then $D \in \mathcal{B}_{E^{\prime}}$ and in this case the assumptions $\mu^{n} \rightarrow \mu$ and $\mu(D)=0$ imply $\mu^{n}\left(\varphi^{n}\right)^{-1} \rightarrow \mu(\varphi)^{-1}$.

Proof. cf. Billingsley (1968) Theorem 5.5.

Remark 82 Notice that if $\varphi^{n}=\varphi$ for all $n \in \mathbb{N}^{+}$, Theorem 81 reduces to the usual continuous mapping theorem, in the sense that if $\varphi$ is $\mu$-a.e. continuous, then $\mu^{n} \rightarrow \mu$ implies $\mu^{n}(\varphi)^{-1} \rightarrow \mu(\varphi)^{-1}$.

Theorem 83 (Skorohod Representation Theorem) Let $(E, d)$ be a separable metric space endowed with the Borel- $\sigma$-algebra $\mathcal{B}_{E}$, and let $\mu,\left(\mu^{n}\right)_{n \in \mathbb{N}}$ be probability measures on $\left(E, \mathcal{B}_{E}\right)$ with $\mu^{n} \rightarrow \mu$. Then there exist a probability space $(\Omega, \mathbb{G}, \mathbb{P})$ and $E$-valued random variables $G$ and $G^{n}$, all defined on $(\Omega, \mathbb{G}, \mathbb{P})$ with distributions $\mu$ and $\mu^{n}$ respectively, and such that $G^{n} \rightarrow G \mathbb{P}$-a.s.

Proof. cf. Ethier and Kurtz (1986), Theorem 2.1.8.

\section{Tightness of Sequence of càdlàg Processes}

Let us consider the Polish space $(E, \mathcal{E})$ with its Borel $\sigma$-field $\mathcal{E}$. Consider the space $\mathcal{P}(E)$ of all probability measures on $(E, \mathcal{E})$ with the weak topology.

Definition $84 A$ subset $A$ of $\mathcal{P}(E)$ is called uniformly tight in $E$ if for every $\varepsilon$ there exists a compact subset $K$ in $E$ such that $\mu(E-K) \leq \varepsilon$ for all $\mu \in A$.

Then, the Prohorov Theorem reads as follows

Theorem 85 (Prohorov) A subset $A$ of $\mathcal{P}(E)$ is relatively compact (for the weak topology) if and only if it is uniformly tight.

Proof. cf. Billingsley (1968) Theorem 5.1.

In this subsection we consider only $\mathbb{R}^{d}$-valued càdlàg processes. Let $G$ be such a process, defined on a triple $(\Omega, \mathbb{G}, \mathbb{P})$. Then it may be considered as a random variable taking its values in the Polish space $\mathbb{D}\left(\mathbb{R}^{d}\right)$. Consequently its law $\mu=\mathcal{L}(G)$ is an element of $\mathcal{P}\left(\mathbb{D}\left(\mathbb{R}^{d}\right)\right)$.

Definition 86 A sequence $\left(G^{n}\right)_{n \in \mathbb{N}}$ is said to be uniformly tight if for every $\varepsilon>0$ there exists a compact set $K$ in $E$ such that $\mathbb{P}\left[G^{n} \notin K\right] \leq \varepsilon$ for all $n \in \mathbb{N}$

Remark 87 Notice that using Prohorov's theorem, we can conclude that the sequence $\left\{\mathcal{L}\left(G^{n}\right)\right\}$ is relatively compact in $\mathcal{P}\left(\mathbb{D}\left(\mathbb{R}^{d}\right)\right)$ if and only if the sequence $\left(G^{n}\right)_{n \in \mathbb{N}}$ is uniformly tight.

The next result is concerned with tightness of sequences of càdlàg processes. Recall that in the Skorohod space we have the following modulus of continuity. Let us fix $T^{\star}>0$ such that $I=$

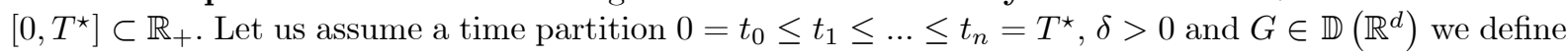

$$
\mathbb{L}_{\delta, t_{n}}(G)=\inf \left\{\max _{i \leq n} \mathbb{L}\left(G ;\left[t_{i-1}, t_{i}\right)\right): n \in \mathbb{N}_{+}, 0=t_{0}<\ldots<t_{n}=T^{\star}, \inf _{i<n}\left(t_{i}-t_{i-1}\right) \geq \delta\right\}
$$

where $\mathbb{L}(G ; I)=\sup _{s, t \in I}|G(s)-G(t)|$ for an interval $I=\left[0, T^{\star}\right] \subset \mathbb{R}_{+}$. 
Theorem 88 Let $\left(G^{n}\right)_{n \in \mathbb{N}}$ be a sequence of càdlàg processes. Then $\left(\mathcal{L}\left(G^{n} \mid \mathbb{P}_{n}\right)\right)_{n \in \mathbb{N}}$ is tight if and only if the following two conditions hold:

(i) for all $T^{\star} \in \mathbb{R}_{+}, \varepsilon>0$ there exist $n_{0} \in \mathbb{N}_{+}, K>0$ such that for all $n \geq n_{0}$

$$
\mathbb{P}_{n}\left[\sup _{t \leq t_{n}}\left|G^{n}\right|>K\right] \leq \varepsilon
$$

(ii) for all $T^{\star} \in \mathbb{R}_{+}, \varepsilon>0, \delta>0$ there exist $n_{0} \in \mathbb{N}_{+}, \theta>0$ such that for all $n \geq n_{0}$

$$
\mathbb{P}_{n}\left[\mathbb{L}_{t_{n}}\left(G^{n}, \theta\right) \geq \delta\right] \leq \varepsilon
$$

Proof. cf. Jacod and Shiryaev (1987) Theorem VI.3.21.

\section{Convergence Results for Sequences of Semimartingale}

Concerning limit theorems for stochastic processes, it is necessary to introduce characteristics of semimartingales, a concept heavily used later in the following theorems. The idea is to associate to a semimartingale a triplet of predictable processes which describe drift, volatility and jumps, in analogy to the concept of characteristic triplet of infinitely divisible distributions, which in turn describes drift, volatility and jumps of the associated Lévy process. The reader should notice that any theorem related with weak convergence of stochastic processes will be necessarily related with semimartingales theory. In fact, in the following sections, we show that if the characteristics triplet of a sequence of the LIBOR additive processes (semimartingales) are known, one can show convergence in distribution via convergence of the characteristics.

First of all, let us assume a time partition $t_{0} \leq t_{1} \leq \ldots \leq t_{n}$ of $\left[0, T^{\star}\right]$ and let us denote with $G^{n}$ a $d$ dimensional semimartingale with independent increments $(P I I)$. On the other hand, $G$ is a $d$-dimensional $P I I$ without fixed time of discontinuity. Then the distribution of the process $G$ is characterized by a triplet of characteristics $(\gamma, A, v)$ relative to some fixed truncation function $h$, or in other words, let $G$ be a semimartingale and $h$ a truncation function and define the process $G(h)$ by

$$
G(h)_{t}=G_{t}-\sum_{s \leq t}\left(\Delta G_{s}-h\left(\Delta G_{s}\right)\right)
$$

Note that $\sum_{s \leq t}\left(\Delta G_{s}-h\left(\Delta G_{s}\right)\right)=\int_{0}^{t}(g-h(g)) \mu^{G}(d s)$ where $\mu^{G}$ is the random measure associated with the jumps of $G$, and since $\Delta G_{s}-h\left(\Delta G_{s}\right) \neq 0$ only for finitely many $s$, this sum converges. Furthermore $\Delta G_{s}-h\left(\Delta G_{s}\right)$ is bounded so $G(h)$ is a special semimartingale with canonical decomposition

$$
G(h)=G_{0}+M(h)+\gamma(h)
$$

where $M(h)$ is a local $\mathbb{P}$-martingale and $\gamma(h)$ is a predictable process with finite variation. Therefore, the triplet $(\gamma, A, v)$ with

$$
\begin{array}{ll}
\gamma=\gamma(h) & \text { from the canonical decomposition } \\
A=\left(\left\langle\tilde{G}_{i}, \tilde{G}_{j}\right\rangle\right)_{1 \leq i, j \leq d} & \text { where } \tilde{G} \text { is the continuous part of } G \\
v=v^{P} & \text { is the } \mathbb{P} \text {-compensator of } \mu^{G}
\end{array}
$$

is called the triplet of $\mathbb{P}$-characteristics of $G$ relative to the truncation function $h$ or simply characteristics if there is no ambiguity about the measure and the truncation function involved. Sometimes $\gamma$ is called the first, $A$ the second, and $v$ the third characteristic of $X$. 
Obviously only the first and the modified second characteristic depend on the choice of the truncation function. In the sequel we fix one truncation function and sometimes do not mention the dependence of the characteristics on this truncation function.

Concerning limit theorems for stochastic processes, it is necessary to define the modified second characteristic of $G, \tilde{A}^{n}$ which is càdlàg and increasing in the set of all $d \times d$ symmetric nonnegative matrices for their natural order, by

$$
\tilde{A}_{i j}^{n}=\left\langle M(h)^{i}, M(h)^{j}\right\rangle
$$

where $M(h)$ is the martingale part in the usual decomposition of a semimartingale ${ }^{14}$.

Recall that we have mentioned that $G$ has no fixed time of discontinuity, it means that $\mu\left(\{t\} \times \mathbb{R}^{d}\right)=0$ and $\tilde{A}, \gamma^{n}(t)$ and $\varphi \cdot \nu$ are continuous functions, then we can state the following theorem ${ }^{15}$

Theorem 89 Let $G^{n}, G$ be $\mathbb{R}^{d}$-valued processes with independent increments and characteristics $\left(\gamma^{n}(t), A^{n}, \nu^{n}\right)$ and $(A, \nu, \gamma(t))$ respectively. Let $\tilde{A}^{n}$ and $\tilde{A}$ be the modified second characteristics of $G^{n}$ and $G$ respectively, and let $D$ be a dense subset of $\mathbb{R}_{+}$. Then $G^{n} \stackrel{\mathcal{L}}{\rightarrow} G$ if and only if the following three conditions hold:

(i) $\sup _{s \leq t}\left|\gamma^{n}(s)-\gamma(s)\right| \rightarrow 0$ for all $t \geq 0$

(ii) $\left|\tilde{A}_{t}^{n}-\tilde{A}_{t}\right| \rightarrow 0$ for all $t \in D$

(iii) $\int_{\mathbb{R}^{d}} \varphi \cdot v_{t}^{n} d g \rightarrow \int_{\mathbb{R}^{d}} \varphi \cdot v_{t} d g$ for all $t \in D, \varphi \in C\left(\mathbb{R}^{d}\right)$

where

$$
C\left(\mathbb{R}^{d}\right):=\left\{f \in \mathcal{C}_{b}\left(\mathbb{R}^{d}\right) ; \exists \varepsilon>0 \forall g \in U_{\varepsilon}(0) f(g)=0 \text { and } \lim _{|g| \rightarrow \infty} f(g) \text { exists }\right\}
$$

and $\mathcal{C}_{b}\left(\mathbb{R}^{d}\right)$ is the class of all continuous and bounded functions $f: \mathbb{R}^{d} \rightarrow \mathbb{R}$.

Proof. cf. Jacod and Shiryaev (1987) Theorem VII.3.4.

\section{Convergence of Stochastic Integrals and Stochastic Differential Equations}

In many cases the semimartingales under consideration are stochastic integrals or solutions of stochastic differential equations driven by a converging sequence of semimartingales. Then one is faced with the question whether the convergence carries over to these new processes. The discussion of this issue dates back to Wong and Zakai (1965) and has received growing interest for obvious reasons. Slominski (1989), Jakubowski, Mémin and Pagès (1989) and Kurtz and Protter (1991) established sufficient conditions for the convergence of stochastic integrals and solutions of stochastic differential equations in terms of uniform tightness of the converging processes, which have the drawback that they are not easy to formulate and sometimes hard to verify. Duffie and Protter (1992) introduce the notion of goodness of a sequence of semimartingales and state simple (but not very general) sufficient conditions. See Kurtz and Protter (1996) for more general results.

\footnotetext{
${ }^{14}$ See Jacod and Shiryaev (1987) Proposition II.2.17

${ }^{15}$ This Theorem can be relaxed to a wider class of functions, however it will be sufficient for our purposes.
} 
For a sequence $\left(\Omega^{n}, \mathbb{F}^{n}, \mathbb{P}^{n}\right)$, let $\left(G^{n}\right)_{n \in \mathbb{N}}$ and $\left(H^{n}\right)_{n \in \mathbb{N}}$ be sequences of càdlàg processes where each $G^{n}$ is an $\mathbb{R}^{d}$-valued $\left(\mathbb{F}^{n}, \mathbb{P}^{n}\right)$-semimartingale and $H^{n}$ is $\mathbb{F}^{n}$-adapted and takes values in $\mathbb{R}^{d^{\prime} \times d}$. Recall that the total variation process of a process $A$ of finite variation is denoted by $\operatorname{Var}(A)=\int|d A|$.

Definition 90 A sequence $\left(G^{n}\right)_{n \in \mathbb{N}}$ of semimartingales is called good (with respect to $\left(\mathbb{P}^{n}\right)_{n \in \mathbb{N}}$ and $\mathbb{P}$ ) if for any sequence $\left(H^{n}\right)_{n \in \mathbb{N}}$ the convergence of $\mathcal{L}\left(G^{n},\left.H^{n}\right|_{\mathbb{P}^{n}}\right) \stackrel{w}{\rightarrow} \mathcal{L}\left(G,\left.H\right|_{\mathbb{P}}\right)$ implies convergence of $\mathcal{L}\left(G^{n}, H^{n},\left.\int H_{-}^{n} d G^{n}\right|_{\mathbb{P}^{n}}\right) \stackrel{w}{\rightarrow} \mathcal{L}\left(G, H,\left.\int H_{-} d G\right|_{\mathbb{P}}\right)$.

Proposition 91 Let $\left(G^{n}\right)_{n \in \mathbb{N}}$ be good and suppose $\mathcal{L}\left(G^{n},\left.H^{n}\right|_{\mathbb{P}^{n}}\right) \stackrel{w}{\rightarrow} \mathcal{L}\left(G,\left.H\right|_{\mathbb{P}}\right)$. Then $\left(\int H_{-}^{n} d G^{n}\right)$ is also good.

Proof. cf. Duffie and Protter (1992) Theorem 4.1.

We next provide a sufficient condition for the convergence of solutions of stochastic differential equations.

Theorem 92 Let $\left(G^{n}\right)_{n \in \mathbb{N}}$ be good, let $G$ be a semimartingale, and let $f: \mathbb{R}_{+} \times \mathbb{R}^{d^{\prime}} \rightarrow \mathbb{R}^{d^{\prime} \times d}$ satisfy

(i) $y \mapsto f(t, y)$ is Lipschitz, uniformly in $t$

(ii) $t \mapsto f(t, y)$ is left-continuous with right-limits, for all $y$

Furthermore let $Y^{n}$ and $Y$ be the (unique) solutions of

$$
\begin{aligned}
d Y_{t}^{n} & =f\left(t, Y_{t-}^{n}\right) d G_{t}^{n}, \quad Y_{t}^{n} \in \mathbb{R}^{d^{\prime}} \\
d Y_{t} & =f\left(t, Y_{t-}\right) d G_{t}, \quad Y_{t} \in \mathbb{R}^{d^{\prime}}
\end{aligned}
$$

If $G^{n} \stackrel{\mathcal{L}}{\rightarrow} G$, then $\left(Y^{n}, G^{n}\right) \stackrel{\mathcal{L}}{\rightarrow}(Y, G)$.

Proof. cf. Duffie and Protter (1992) Theorem 4.4.

\subsubsection{Relative compactness in the space $D_{\left[0, T^{\star}\right]}\left(\mathbb{R}^{d}, \mathcal{K}\right)$}

This section is devoted to study the conditions for relative compactness of a subset in $D_{\left[0, T^{\star}\right]}\left(\mathbb{R}^{d}, \mathcal{K}\right)$. Basically, we look for a result equivalent to Theorem 88 or the Arzelà-Ascoli Theorem for the Skorohod space $D_{\left[0, T^{*}\right]}\left(\mathbb{R}^{d}, \mathcal{K}\right)$. In the sequel, we consider for any $n \geq 1$, a semimartingale $G^{n, C}$ that depends on a process $C$, and defined on the stochastic bases $\left(\Omega^{n}, \mathbb{F}^{n}, \mathbb{P}^{n}\right)$. The main aim of this subsection is to find conditions such that $\left(G^{n, C}\right)_{n \in \mathbb{N}}$ are relatively compact in $D_{\left[0, T^{\star}\right]}\left(\mathbb{R}^{d}, \mathcal{K}\right)$.

Let us begin recalling some definitions:

- Let $\Pi_{\delta}^{(n)}$ be the partition of time $\left\{0=t_{0}<t_{1}<\ldots<t_{n} \leq T^{\star} \leq 1\right\}$ where $n \in \mathbb{N}_{+}$, satisfying the condition $\min _{0 \leq j \leq n-1}\left(t_{j}-t_{j-1}\right)>\delta$ on the "normalized" interval $[0,1]$

- And let $\mathcal{K}^{(m)}$ be the set of credit-ratings $\left\{0=C_{0}<C_{1}<\ldots<C_{m}=1\right\}$ where $m \in \mathbb{N}_{+}$. 
Let us define the following moduli of continuity in $D_{\left[0, T^{\star}\right]}\left(\mathbb{R}^{d}, \mathcal{K}\right)$

$$
\begin{aligned}
\mathbb{L}_{\delta}^{(m)}(G) & =\inf _{\substack{\Pi_{\delta}^{(n)} \\
t_{j+1}-t_{j}>\delta}} \max _{0 \leq j \leq n} \sup _{s, t \in\left[t_{j}, t_{j+1}\right)} \sup _{c \in \mathcal{K}^{(m)}}\left|G_{s}^{c}-G_{t}^{c}\right| \\
& \mathbb{L} \mathbb{L}_{\delta}^{(m)}(G)=\sup _{0 \leq t \leq 1} \sup _{C^{\prime}, C \in \mathcal{K}^{(m)}}\left|G_{t}^{C^{\prime}}-G_{t}^{C}\right|
\end{aligned}
$$

with $n, m \in \mathbb{N}_{+}, T^{\star} \in \mathbb{R}_{+}$and $\delta \in \mathbb{R}_{+}$.

Additionally, let us define another modulus of continuity in $D_{\left[0, T^{\star}\right]}\left(\mathbb{R}^{d}, \mathcal{K}\right)$

$$
\mathbb{L}_{\delta}^{C}(G)=\inf _{\substack{\Pi_{\delta}^{(n)} \\ t_{j+1}-t_{j}>\delta}} \max _{0 \leq j \leq n} \sup _{s, t \in\left[t_{j}, t_{j+1}\right)}\left|G_{s}^{C}-G_{t}^{C}\right|
$$

Basically, for the most part, we are concerned with the relative compactness of sequences $\left\{\mathbb{P}_{n}\right\}$; this

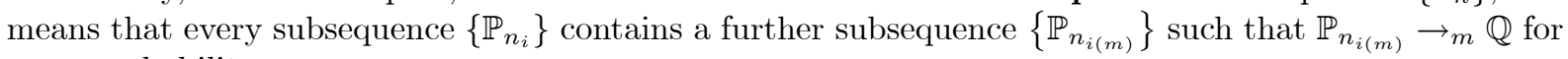
some probability measure.

Theorem 93 Let us assume $C \in \mathcal{K}^{(m)}, n, m \in \mathbb{N}_{+}$, and $a, \varepsilon>0$. Additionally, assume that the following conditions hold

1)

$$
\lim _{a \rightarrow+\infty} \varlimsup_{n \rightarrow \infty} \mathbb{P}_{n}\left(\sup _{0 \leq t \leq T^{\star}} \sup _{C \in \mathcal{K}^{(m)}}\left|G_{t}^{n, C}\right| \geq a\right)=0
$$

2)

$$
\lim _{\delta \rightarrow 0} \varlimsup_{n \rightarrow \infty} \mathbb{P}_{n}\left(\mathbb{L}_{\delta}^{C}\left(G^{n, C}\right)>\varepsilon\right)=0
$$

3)

$$
\lim _{\delta \rightarrow 0} \sup _{n \geq 1} \mathbb{P}_{n}\left(\mathbb{L L}_{\delta}^{(m)}\left(G^{n}\right)>\varepsilon\right)=0
$$

Then the processes $G^{n, C}$ with $n \geq 1$ have sample-paths in the Skorohod space $D_{\left[0, T^{\star}\right]}\left(\mathbb{R}^{d}, \mathcal{K}\right)$, and conditions 1), 2) and 3) hold if and only if the set of processes $\left(G^{n, C}\right)_{n \geq 1}$ are relatively compact in $D_{\left[0, T^{\star}\right]}\left(\mathbb{R}^{d}, \mathcal{K}\right)$.

In order to prove the Theorem 93, it will be useful to prove the following result.

Proposition 94 Assuming condition 3) in Theorem 93, then the condition 2) in Theorem 93 is satisfied if and only if the following expression

$$
\lim _{\delta \rightarrow 0} \varlimsup_{n \rightarrow+\infty} \mathbb{P}_{n}\left(\sup _{C \in \mathcal{K}^{(m)}} \mathbb{L}_{\delta}^{C}\left(G^{n, C}\right)>\varepsilon\right)=0
$$

is true for every $m \in \mathbb{N}_{+}$.

Proof. It is obvious that (2.55) implies the condition 2) in Theorem 93. Conversely, assuming that conditions 2) and 3) in Theorem 93 are true, let us prove that both implies (2.55). 
Fix a $\eta>0$. Using directly condition 3) in Theorem 93, there is a $\delta^{\prime}>0$ such that

$$
\sup _{n \geq 1} \mathbb{P}_{n}\left(\mathbb{L L}_{\delta^{\prime}}^{(m)}\left(G^{n}\right)>\frac{\varepsilon}{4}\right) \leq \frac{\eta}{2}
$$

Following the same notation that in Lemma 78, fix any $m \in \mathbb{N}_{+}$, then $\mathcal{K}^{(m)}$ can be covered by a finite union of open-balls with radius $\delta^{\prime}$. Therefore there are $C_{1}, \ldots, C_{r\left(\delta^{\prime}\right)}$ in $\mathcal{K}^{(m)}$ such that $B\left(C_{k}, \delta^{\prime}\right)$ with $k=1, \ldots, r\left(\delta^{\prime}\right)$

$$
\mathcal{K}^{(m)} \subseteq B\left(C_{1}, \delta^{\prime}\right) \cup \ldots \cup B\left(C_{r\left(\delta^{\prime}\right)}, \delta^{\prime}\right)
$$

On the other hand, assuming that the condition 2) in Theorem 93 is satisfied, there is a $\delta^{\prime \prime}>0$ such that for every $k=1, \ldots, r\left(\delta^{\prime}\right)$

$$
\varlimsup_{n \rightarrow \infty} \mathbb{P}_{n}\left(\mathbb{L}_{\delta^{\prime \prime}}^{C_{k}}\left(G^{n}\right)>\frac{\varepsilon}{4}\right) \leq \frac{\eta}{2 r\left(\delta^{\prime}\right)}
$$

Using directly Lemma 78, there exists a $\delta^{0}$ such that $0<\delta^{0} \leq \delta^{\prime \prime}$ and

$$
\sup _{C \in \mathcal{K}^{(m)}} \mathbb{L}_{\delta^{0}}^{C}\left(G^{n}\right) \leq 2 \max _{1 \leq k \leq r\left(\delta^{\prime}\right)} \mathbb{L}_{\delta^{\prime \prime}}^{C_{k}}\left(G^{n}\right)+2 \mathbb{L} \mathbb{L}_{\delta^{\prime}}^{(m)}\left(G^{n}\right)
$$

notice that $\mathbb{L}_{\delta}^{C}\left(G^{n, C}\right)$ is an increasing function of $\delta$, therefore we have for every $\delta \leq \delta^{0}$

$$
\mathbb{P}_{n}\left(\sup _{C \in \mathcal{K}^{(m)}} \mathbb{L}_{\delta}^{C}\left(G^{n}\right)>\varepsilon\right) \leq \sum_{k=1}^{r\left(\delta^{\prime}\right)} \mathbb{P}_{n}\left(\mathbb{L}_{\delta^{\prime \prime}}^{C_{k}}\left(G^{n}\right)>\frac{\varepsilon}{4}\right)+\mathbb{P}_{n}\left(\mathbb{L L}_{\delta^{\prime}}^{(m)}\left(G^{n}\right)>\frac{\varepsilon}{4}\right)
$$

Finally taking into account the expressions (2.56), (2.57) and (2.58) we obtain for every $\delta<\delta^{0}$

$$
\varlimsup_{n \rightarrow+\infty} \mathbb{P}_{n}\left(\sup _{C \in \mathcal{K}^{(m)}} \mathbb{L}_{\delta}^{C}\left(G^{n, C}\right)>\varepsilon\right) \leq \eta
$$

which implies (2.55) and the proof is complete.

Proof. (Theorem 93) Let us first prove that if conditions 1), 2) and 3) hold then $G^{n, C}$ have samplepaths in the Skorohod space $D_{\left[0, T^{\star}\right]}\left(\mathbb{R}^{d}, \mathcal{K}\right)$.

We know that the process $G^{n, C}$, defined in $\left(\Omega^{n}, \mathbb{F}^{n}, \mathbb{P}^{n}\right)$, has sample-paths in $D_{\left[0, T^{\star}\right]}\left(\mathbb{R}^{d}, \mathcal{K}\right)$ if and only if

or, equivalently

$$
\mathbb{P}_{n}\left(\left\{\omega \in \Omega^{n}, G^{n}(\omega) \in D_{\left[0, T^{\star}\right]}\left(\mathbb{R}^{d}, \mathcal{K}\right)\right\}\right)=1
$$

$$
\mathbb{P}_{n}\left(\left\{\omega \in \Omega^{n}, G^{n}(\omega) \notin D_{\left[0, T^{\star}\right]}\left(\mathbb{R}^{d}, \mathcal{K}\right)\right\}\right)=0
$$

and, using directly Theorem 75, we have that

$$
\begin{aligned}
& \left\{\omega \in \Omega^{n}, G^{n}(\omega) \notin D_{\left[0, T^{\star}\right]}\left(\mathbb{R}^{d}, \mathcal{K}\right)\right\} \\
= & \left\{\omega \in \Omega^{n}, \exists C \in \mathcal{K}, \lim _{\delta \rightarrow 0} \mathbb{L}_{\delta}^{C}\left(G^{n}\right)>0\right\} \cup\left\{\omega \in \Omega^{n}, \exists m \in \mathbb{N}, \lim _{\delta \rightarrow 0} \mathbb{L} \mathbb{L}_{\delta}^{(m)}\left(G^{n}\right)>0\right\} \\
\subseteq & \left\{\omega \in \Omega^{n}, \exists m \in \mathbb{N}, \lim _{\delta \rightarrow 0} \sup _{C \in \mathcal{K}^{(m)}} \mathbb{L}_{\delta}^{C}\left(G^{n}\right)>0\right\} \cup\left\{\omega \in \Omega^{n}, \exists m \in \mathbb{N}, \lim _{\delta \rightarrow 0} \mathbb{L L}_{\delta}^{(m)}\left(G^{n}\right)>0\right\}
\end{aligned}
$$


Therefore, our goal is to prove that

$$
\mathbb{P}_{n}\left(\left\{\omega \in \Omega^{n}, \lim _{\delta \rightarrow 0} \sup _{C \in \mathcal{K}^{(m)}} \mathbb{L}_{\delta}^{C}\left(G^{n}\right)>0\right\}\right)=0
$$

and

$$
\mathbb{P}_{n}\left(\left\{\omega \in \Omega^{n}, \lim _{\delta \rightarrow 0} \mathbb{L}_{\delta}^{(m)}\left(G^{n}\right)>0\right\}\right)=0
$$

Let us begin with (2.59). Notice that (2.59) can be proved using the following equivalence

$$
\mathbb{P}_{n}\left(\left\{\omega \in \Omega^{n}, \lim _{\delta \rightarrow 0} \sup _{C \in \mathcal{K}^{(m)}} \mathbb{L}_{\delta}^{C}\left(G^{n}\right)>0\right\}\right)=\lim _{k \rightarrow+\infty} \mathbb{P}_{n}\left(\left\{\omega \in \Omega^{n}, \lim _{\delta \rightarrow 0} \sup _{C \in \mathcal{K}^{(m)}} \mathbb{L}_{\delta}^{C}\left(G^{n}\right)>\frac{1}{k}\right\}\right)
$$

and noting that $\mathbb{L}_{\delta}^{C}\left(G^{n}\right)$ is an increasing function of $\delta$ therefore we have

$$
\lim _{k \rightarrow+\infty} \mathbb{P}_{n}\left(\left\{\omega \in \Omega^{n}, \lim _{\delta \rightarrow 0} \sup _{C \in \mathcal{K}^{(m)}} \mathbb{L}_{\delta}^{C}\left(G^{n}\right)>\frac{1}{k}\right\}\right) \leq \lim _{k \rightarrow+\infty} \lim _{\delta \rightarrow 0} \mathbb{P}_{n}\left(\left\{\omega \in \Omega^{n}, \sup _{C \in \mathcal{K}^{(m)}} \mathbb{L}_{\delta}^{C}\left(G^{n}\right)>\frac{1}{k}\right\}\right)
$$

Notice that for every $n \geq 1$ and for every $C \in \mathcal{K}^{(m)}$, the process $G^{n, C}$ is a semimartingale and it has sample-paths in the Skorohod space $D_{\left[0, T^{\star}\right]}\left(\mathbb{R}^{d}, \mathcal{K}\right)$. Therefore we have, for every $k \in \mathbb{N}_{+}$(cf. Liptser and Shiryayev (1986) Theorem 6.1.6).

$$
\mathbb{P}_{n}\left(\left\{\omega \in \Omega^{n}, \mathbb{L}_{\delta}^{C}\left(G^{n}\right)>\frac{1}{k}\right\}\right)=0
$$

Notice that following the same reasoning than in Proposition 94 we can show that the equality (2.61) and the condition 3) in Theorem 93 implies for every $k \in \mathbb{N}_{+}$

$$
\mathbb{P}_{n}\left(\left\{\omega \in \Omega^{n}, \sup _{C \in \mathcal{K}^{(m)}} \mathbb{L}_{\delta}^{C}\left(G^{n}\right)>\frac{1}{k}\right\}\right)=0
$$

and notice that when $k \rightarrow+\infty$ we obtain $(2.59)$.

Similarly, we can prove (2.60) following a similar reasoning, noting also that $\mathbb{L}_{\delta}^{(m)}\left(G^{n}\right)$ is an increasing function of $\delta$,

$$
\mathbb{P}_{n}\left(\left\{\omega \in \Omega^{n}, \lim _{\delta \rightarrow 0} \mathbb{L}_{\delta}^{(m)}\left(G^{n}\right)>0\right\}\right) \leq \lim _{k \rightarrow+\infty} \lim _{\delta \rightarrow 0} \mathbb{P}_{n}\left(\left\{\omega \in \Omega^{n}, \mathbb{L}_{\delta}^{(m)}\left(G^{n}\right)>\frac{1}{k}\right\}\right)
$$

where

$$
\lim _{\delta \rightarrow 0} \mathbb{P}_{n}\left(\left\{\omega \in \Omega^{n}, \mathbb{L}_{\delta}^{(m)}\left(G^{n}\right)>\frac{1}{k}\right\}\right)=0
$$

and finally, we conclude that the process $G^{n}$ has sample-paths in the space $D_{\left[0, T^{\star}\right]}\left(\mathbb{R}^{d}, \mathcal{K}\right)$.

In the second part, we have to show that these three conditions in Theorem 93 are necessary and sufficient conditions to have relative compactness of $\left(G^{n, C}\right)_{n \geq 1}$ in $D_{\left[0, T^{\star}\right]}\left(\mathbb{R}^{d}, \mathcal{K}\right)$.

According to Theorem VI.1.14 in Jacod and Shiryaev (1989), a subset $A$ of $D_{\left[0, T^{\star}\right]}\left(\mathbb{R}^{d}, \mathcal{K}\right)$ is 
relatively compact for the Skorohod topology for every $m \in \mathbb{N}_{+}$and for a fixed $T^{\star}$ if and only if

$$
\begin{aligned}
& \text { 1) } \sup _{G \in A} \sup _{0 \leq t \leq T^{\star}} \sup _{C \in \mathcal{K}^{(m)}}\left|G_{t}^{C}\right|<+\infty \\
& \text { 2) } \lim _{\delta \rightarrow 0} \sup _{G \in A} \mathbb{L}_{\delta}^{(m)}(G)=0
\end{aligned}
$$

Notice that using a similar reasoning as in Theorem 75, condition 2) can be split into these two equivalent conditions

$$
\begin{aligned}
& \text { 1) } \lim _{\delta \rightarrow 0} \sup _{G \in A} \sup _{C \in \mathcal{K}^{(m)}} \mathbb{L}_{\delta}^{C}(G)=0 \\
& \text { 2) } \lim _{\delta \rightarrow 0} \sup _{G \in A} \mathbb{L}_{\delta}^{(m)}(G)=0
\end{aligned}
$$

Therefore we can show that a subset $A$ of $D_{\left[0, T^{\star}\right]}\left(\mathbb{R}^{d}, \mathcal{K}\right)$ is relatively compact for the Skorohod topology for every $m \in \mathbb{N}_{+}$and for a fixed $T^{\star}$ if and only if

$$
\begin{array}{ll}
\text { 1) } & \sup _{G \in A} \sup _{0 \leq t \leq T^{\star}} \sup _{C \in \mathcal{K}^{(m)}}\left|G_{t}^{C}\right|<+\infty \\
\text { 2) } & \lim _{\delta \rightarrow 0} \sup _{G \in A} \sup _{C \in \mathcal{K}^{(m)}} \mathbb{L}_{\delta}^{C}(G)=0 \\
\text { 3) } & \lim _{\delta \rightarrow 0} \sup _{G \in A} \mathbb{L} \mathbb{L}_{\delta}^{(m)}(G)=0
\end{array}
$$

Now we prove that conditions 1), 2) and 3) in Theorem 93 are necessary and sufficient to guarantee than $\left(G^{n}\right)_{n \geq 1}$ is relatively compact in $D_{\left[0, T^{\star}\right]}\left(\mathbb{R}^{d}, \mathcal{K}\right)$.

Notice that using Prohorov Theorem (Theorem 85), if the subset $A$ is relatively compact, then it is tight. Let us assume that the set $\left(G^{n}\right)_{n \geq 1}$ is relatively compact and let us give a $\varepsilon>0$. Therefore, according to Prohorov Theorem, there exist a compact $A_{\varepsilon}$ in $D_{\left[0, T^{\star}\right]}\left(\mathbb{R}^{d}, \mathcal{K}\right)$ such that if $\bar{A}_{\varepsilon}$ is the complement of $A_{\varepsilon}$ then we have for any $n \geq 1$

$$
\mathbb{P}_{n}\left(\bar{A}_{\varepsilon}\right) \leq \varepsilon
$$

where $\mathbb{P}_{n}$ is the law of $G^{n}$ in the space $D_{\left[0, T^{\star}\right]}\left(\mathbb{R}^{d}, \mathcal{K}\right)$. Then, using the set of conditions (2.62), and replacing $A$ by $A_{\varepsilon}$ we have that for any $C \in \mathcal{K}^{(m)}$ with $m \in \mathbb{N}_{+}$, with a fixed $T^{\star}$, and any $\eta>0$ there exist $a_{m, T^{\star}}, \delta_{m, T^{\star}, \eta} \in \mathbb{R}$ such that

$$
\begin{aligned}
& \text { 1) } A_{\varepsilon} \subseteq\left\{G^{C} \in D_{\left[0, T^{\star}\right]}\left(\mathbb{R}^{d}, \mathcal{K}\right), \sup _{0 \leq t \leq T^{\star}} \sup _{C \in \mathcal{K}^{(m)}}\left|G_{t}^{C}\right| \leq a_{m, T^{\star}}\right\} \\
& \text { 2) } A_{\varepsilon} \subseteq\left\{G^{C} \in D_{\left[0, T^{\star}\right]}\left(\mathbb{R}^{d}, \mathcal{K}\right), \sup _{C \in \mathcal{K}^{(m)}} \mathbb{L}_{\delta_{m, T^{\star}, \eta}^{C}}(G) \leq \eta\right\} \\
& \text { 3) } A_{\varepsilon} \subseteq\left\{G^{C} \in D_{\left[0, T^{\star}\right]}\left(\mathbb{R}^{d}, \mathcal{K}\right), \mathbb{L}_{\delta_{m, T^{\star}, \eta}^{(m)}}^{(m)}(G) \leq \eta\right\}
\end{aligned}
$$

and for any $C \in \mathcal{K}^{(m)}$, with a fixed $T^{\star}$ and any $\eta>0$, we finally obtain conditions 1 ), 2) and 3) in 
Theorem 93

$$
\begin{aligned}
& \text { 1) } \sup _{n \geq 1} \mathbb{P}_{n}\left(\sup _{0 \leq t \leq T^{\star}} \sup _{C \in \mathcal{K}^{(m)}}\left|G_{t}^{n, C}\right| \leq a_{m, T^{\star}}\right) \leq \varepsilon \\
& \text { 2) } \sup _{n \geq 1} \mathbb{P}_{n}\left(\sup _{C \in \mathcal{K}^{(m)}} \mathbb{L}_{\delta_{m, T^{\star}, \eta}^{C}}^{C}\left(G^{n}\right) \leq \eta\right) \leq \varepsilon \\
& \text { 3) } \sup _{n \geq 1} \mathbb{P}_{n}\left(\mathbb{L}_{\delta_{\delta_{m, T^{\star}, \eta}}^{(m)}}\left(G^{n}\right) \leq \eta\right) \leq \varepsilon
\end{aligned}
$$

Conversely, in order to prove the 'only if' part of this theorem, let us assume the conditions 1), 2) and 3) holds, for any $C \in \mathcal{K}^{(m)}$, with a fixed $T^{\star}$ and any $\eta>0$, and for any $n \geq 1$. Let $\mathbb{P}_{n}$ be the law of $G^{n}$ in $D_{\left[0, T^{\star}\right]}\left(\mathbb{R}^{d}, \mathcal{K}\right)$. Notice that using condition 1$)$ we can obtain some results about tightness, or more specifically, there is an $a_{m, T^{*}}$ large enough such that

$$
A=\left\{G^{C} \in D_{\left[0, T^{\star}\right]}\left(\mathbb{R}^{d}, \mathcal{K}\right), \sup _{0 \leq t \leq T^{\star}} \sup _{C \in \mathcal{K}^{(m)}}\left|G_{t}^{C}\right| \leq a_{m, T^{\star}}\right\}
$$

therefore, we have

$$
\varliminf_{n \rightarrow+\infty} \mathbb{P}_{n}(A) \geq 1-\frac{\varepsilon}{3}
$$

And similarly, using conditions 2) and 3) we can assume that exists a $\delta_{m, T^{\star}, k}$ small enough such that

$$
A_{m, T^{\star}, k}=\left\{G^{C} \in D_{\left[0, T^{\star}\right]}\left(\mathbb{R}^{d}, \mathcal{K}\right), \sup _{C \in \mathcal{K}} \mathbb{L}_{\delta_{m, T^{\star}, k}}(G) \leq \frac{1}{k}\right\}
$$

and

$$
B_{m, T^{\star}, k}=\left\{G^{C} \in D_{\left[0, T^{\star}\right]}\left(\mathbb{R}^{d}, \mathcal{K}\right), \mathbb{L}_{\delta_{\delta_{m, T^{\star}, k}}^{(m)}}(G) \leq \frac{1}{k}\right\}
$$

such that

$$
\varliminf_{n \rightarrow+\infty} \mathbb{P}_{n}\left(A_{m, T^{\star}, k}\right) \geq 1-\frac{\varepsilon}{3}
$$

and

$$
\varliminf_{n \rightarrow+\infty} \mathbb{P}_{n}\left(B_{m, T^{\star}, k}\right) \geq 1-\frac{\varepsilon}{3}
$$

Let us define now the set $K$ in $D_{\left[0, T^{\star}\right]}\left(\mathbb{R}^{d}, \mathcal{K}\right)$ as the set $K=A \cap A_{m, T^{\star}, k} \cap B_{m, T^{\star}, k}$, therefore $K$ is a compact set in the space $D_{\left[0, T^{\star}\right]}\left(\mathbb{R}^{d}, \mathcal{K}\right)$, and verify that

$$
\underline{\lim }_{n \rightarrow+\infty} \mathbb{P}_{n}(K) \geq 1-\frac{\varepsilon}{3}-\frac{\varepsilon}{3}-\frac{\varepsilon}{3} \geq 1-\varepsilon
$$

and it shows directly that $\left(G^{n}\right)_{n \geq 1}$ is tight in $D_{\left[0, T^{\star}\right]}\left(\mathbb{R}^{d}, \mathcal{K}\right)$, and relatively compact, according to the Prohorov theorem.

\subsubsection{Weak Convergence in $D_{\left[0, T^{\star}\right]}\left(\mathbb{R}^{d}, \mathcal{K}\right)$}

As in the last subsection, let us consider, for any $n \geq 1$, the semimartingale $G^{n, C}$ with $C \in \mathcal{K}$, defined on 'sufficiently rich' stochastic bases $\left(\Omega^{n}, \mathbb{G}^{n}, \mathbb{P}^{n}\right)$ endowed with the filtration $\mathbb{G}^{n}=\left(\mathcal{G}_{t}^{n}\right)_{t \in\left[0, T^{\star}\right]}$, and additionally let us define the stochastic process $G^{C}$ with $C \in \mathcal{K}$, also in a 'sufficiently rich' stochastic basis $(\Omega, \mathbb{G}, \mathbb{P})$ endowed with the filtration $\mathbb{G}=\left(\mathcal{G}_{t}\right)_{t \in\left[0, T^{\star}\right]}$. This subsection is mainly devoted to find the 
basic conditions in order to obtain a $G^{n, C}$ with sample-paths in $D_{\left[0, T^{\star}\right]}\left(\mathbb{R}^{d}, \mathcal{K}\right)$, and convergence in law to $G^{C}$, in the space $D_{\left[0, T^{\star}\right]}\left(\mathbb{R}^{d}, \mathcal{K}\right)$.

Theorem 95 We assume that the finite-dimensional distributions of $G^{n, C}$ converge weakly to the finitedimensional distributions of $G^{C}$. Let us assume $C \in \mathcal{K}^{(m)}, n, m \in \mathbb{N}_{+}$, and $\varepsilon, a>0$. Additionally, assume that the following conditions hold

1)

$$
\lim _{a \rightarrow+\infty} \varlimsup_{n \rightarrow \infty} \mathbb{P}_{n}\left(\sup _{0 \leq t \leq T^{\star}} \sup _{C \in \mathcal{K}_{\delta}^{(m)}}\left|G_{t}^{n, C}\right| \geq a\right)=0
$$

2)

$$
\lim _{\delta \rightarrow 0} \varlimsup_{n \rightarrow \infty} \mathbb{P}_{n}\left(\mathbb{L}_{\delta}^{C}\left(G^{n}\right)>\varepsilon\right)=0
$$

3)

$$
\lim _{\delta \rightarrow 0} \sup _{n \geq 1} \mathbb{P}_{n}\left(\mathbb{L}_{\delta}^{(m)}\left(G^{n}\right)>\varepsilon\right)=0
$$

Then the processes $G^{n, C}$ with $n \geq 1$ have sample-paths in the Skorohod space $D_{\left[0, T^{\star}\right]}\left(\mathbb{R}^{d}, \mathcal{K}\right)$, and the weak convergence of $G^{n, C} \stackrel{w\left(\mathbb{P}_{n}\right)}{\longrightarrow} G^{C}$ in the Skorohod space $D_{\left[0, T^{\star}\right]}\left(\mathbb{R}^{d}, \mathcal{K}\right)$ takes place.

Proof. First, we have to prove that the process $G^{n, C}$ has sample paths in the Skorohod space $D_{\left[0, T^{\star}\right]}\left(\mathbb{R}^{d}, \mathcal{K}\right)$, and that $\left(G^{n, C}\right)_{n>1}$ is relatively compact. In order to show that, it is enough to see that the conditions in Theorem 95 are the same as the conditions in Theorem 75 and Theorem 93.

Second, we have to prove the weak convergence of the sequence $\left(G^{n, C}\right)_{n \geq 1}$. Let us define $\mathbb{Q}^{n}$ as the law of $G^{n}$ in $D_{\left[0, T^{\star}\right]}\left(\mathbb{R}^{d}, \mathcal{K}\right)$. Also let us consider two subsequences $\left(\mathbb{Q}^{n \prime}\right)$ and $\left(\mathbb{Q}^{n \prime \prime}\right)$, from the sequence $\left(\mathbb{Q}^{n}\right)_{n \geq 1}$, that converge to the probabilities $\mathbb{Q}^{\prime}$ and $\mathbb{Q}^{\prime \prime}$ in the measurable space $D_{\left[0, T^{\star}\right]}\left(\mathbb{R}^{d}, \mathcal{K}\right)$. According to Theorem 2.8 in Billingsley (1968) in order to prove weak convergence, it is enough to prove that $\mathbb{Q}^{\prime}$ and $\mathbb{Q}^{\prime \prime}$ are the same.

For $0 \leq t_{1}<\ldots<t_{k} \leq 1$ with $k \in \mathbb{N}_{+}$, let us define the natural projection from $D\left(\mathbb{R}^{\infty}\right)$ to $\mathbb{R}^{k}$ by $\Pi_{t_{1}, \ldots, t_{k}}(G)=\left(G_{t_{1}}, \ldots, G_{t_{k}}\right)$, such that for $A \in \mathbb{R}^{n}$ we can denote

$$
\Pi_{t_{1}, \ldots, t_{k}}^{-1}(A)=\left\{\left(G_{t_{1}}, \ldots, G_{t_{k}}\right) \in A\right\}
$$

and notice that $\Pi_{t_{1}, \ldots, t_{k}}$ is continuous for all $i=1, \ldots, k, t_{i} \in \Lambda(G)=\left\{t>0, \Delta G_{t}=0\right\} \cup\{0\}$.

Now, let us define

$$
\begin{aligned}
\Lambda_{\mathbb{Q}^{\prime}} & =\left\{t>0: \mathbb{Q}^{\prime}\left(G \in D_{\left[0, T^{\star}\right]}\left(\mathbb{R}^{d}, \mathcal{K}\right), \Delta G_{t}=0\right)=1\right\} \cup\{0\} \\
\Lambda_{\mathbb{Q}^{\prime \prime}} & =\left\{t>0: \mathbb{Q}^{\prime \prime}\left(G \in D_{\left[0, T^{\star}\right]}\left(\mathbb{R}^{d}, \mathcal{K}\right), \Delta G_{t}=0\right)=1\right\} \cup\{0\}
\end{aligned}
$$

Then we can say that, for any $t_{1}, \ldots, t_{k} \in \Lambda_{\mathbb{Q}^{\prime}} \cap \Lambda_{\mathbb{Q}^{\prime \prime}}$, the sequences $\left(\mathbb{Q}^{n \prime} \circ \Pi_{t_{1}, \ldots, t_{k}}^{-1}\right)$ and $\left(\mathbb{Q}^{n \prime \prime} \circ \Pi_{t_{1}, \ldots, t_{k}}^{-1}\right)$ converge weakly to $\left(\mathbb{Q}^{\prime} \circ \Pi_{t_{1}, \ldots, t_{k}}^{-1}\right)$ and $\left(\mathbb{Q}^{\prime \prime} \circ \Pi_{t_{1}, \ldots, t_{k}}^{-1}\right)$ respectively.

Now, let us fix $t_{1}, \ldots, t_{k} \in \Lambda_{\mathbb{Q}^{\prime}} \cap \Lambda_{\mathbb{Q}^{\prime \prime}}$ and $0 \leq C_{1}<\ldots<C_{m} \leq 1$ with $C_{1}, \ldots, C_{m} \in \mathbb{R}_{+}$and $m \in \mathbb{N}_{+}$. Additionally let us define the continuous mapping

$$
\Pi_{C_{1}, \ldots, C_{m}}\left(G_{t_{1}}, \ldots, G_{t_{k}}\right)=\left(G_{t_{1}}^{C_{1}}, \ldots, G_{t_{1}}^{C_{m}}, \ldots, G_{t_{k}}^{C_{1}}, \ldots, G_{t_{k}}^{C_{m}}\right)
$$


from $\mathbb{R}^{k}$ to $\mathbb{R}^{k \cdot m}$. Notice that according to the continuous mapping theorem (Theorem 81 ), the sequences $\left(\mathbb{Q}^{n \prime} \circ \Pi_{t_{1}, \ldots, t_{k}}^{-1} \circ \Pi_{C_{1}, \ldots, C_{m}}^{-1}\right)$ and $\left(\mathbb{Q}^{n \prime \prime} \circ \Pi_{t_{1}, \ldots, t_{k}}^{-1} \circ \Pi_{C_{1}, \ldots, C_{m}}^{-1}\right)$ converge weakly to $\left(\mathbb{Q}^{\prime} \circ \Pi_{t_{1}, \ldots, t_{k}}^{-1} \circ \Pi_{C_{1}, \ldots, C_{m}}^{-1}\right)$ and $\left(\mathbb{Q}^{\prime \prime} \circ \Pi_{t_{1}, \ldots, t_{k}}^{-1} \circ \Pi_{C_{1}, \ldots, C_{m}}^{-1}\right)$ respectively.

Notice that, if we assume that the finite-dimensional distributions of $G^{n, C}$ converge weakly to the finite-dimensional distributions of $G^{C}$, then we have that

$$
\mathbb{Q}^{\prime} \circ \Pi_{t_{1}, \ldots, t_{k}}^{-1} \circ \Pi_{C_{1}, \ldots, C_{m}}^{-1}=\mathbb{Q}^{\prime \prime} \circ \Pi_{t_{1}, \ldots, t_{k}}^{-1} \circ \Pi_{C_{1}, \ldots, C_{m}}^{-1}
$$

Let us prove that, for any $C_{1}, \ldots, C_{m} \in \mathbb{R}_{+}$with $m \in \mathbb{N}_{+}$, we have

$$
\mathbb{Q}^{\prime} \circ \Pi_{t_{1}, \ldots, t_{k}}^{-1}=\mathbb{Q}^{\prime \prime} \circ \Pi_{t_{1}, \ldots, t_{k}}^{-1}
$$

or in other words, if we denote $\mathcal{C}_{k}$ as the $\sigma$-field from $(\mathcal{K})^{k}$, and $\overline{\mathcal{C}}_{k}$ as the $\sigma$-field from $(\mathcal{K})^{k}$ generated by $\Pi_{C_{1}, \ldots, C_{m}}^{-1}(A)$, with $C_{1}, \ldots, C_{m} \in \mathbb{R}_{+}, m \in \mathbb{N}_{+}$and $A \in \mathcal{B}\left(\mathbb{R}^{k \cdot m}\right)$, then we have to prove that $\mathcal{C}_{k}=\overline{\mathcal{C}}_{k}$.

Notice first that, for any $C_{1}, \ldots, C_{m} \in \mathbb{R}_{+}$, the application $\Pi_{C_{1}, \ldots, C_{m}}$ such that

$$
\Pi_{C_{1}, \ldots, C_{m}}: G=\left(G_{1}, \ldots, G_{k}\right) \rightarrow\left(G_{1}^{C_{1}}, \ldots, G_{1}^{C_{m}}, \ldots, G_{k}^{C_{1}}, \ldots, G_{k}^{C_{m}}\right)
$$

is continuous. Therefore $\overline{\mathcal{C}}_{k} \subseteq \mathcal{C}_{k}$.

On the other hand, in order to show the inclusion in the opposite direction, it is enough to show that any ball in $(\mathcal{K})^{k}$ is $\overline{\mathcal{C}}_{k}$-measurable, because $\mathcal{K}$ is a complete and separable space. Let us choose $C$ from $C_{1}, \ldots, C_{r(\delta)}$ in the compact $\mathcal{K}_{i}$ such that $\mathcal{K}_{i} \subset B\left(C_{1}, \delta\right) \cup \ldots \cup B\left(C_{r(\delta)}, \delta\right)$ and such that $r(\delta)$ is the minimum number of balls with radius $\delta$ that cover $\mathcal{K}_{i}$. Therefore let us define, the following $\overline{\mathcal{C}}_{1}$-measurable application with

$$
G \rightarrow d(G, Y)=\sum_{i=0}^{+\infty} 2^{-i} \frac{\sup _{C \in \mathcal{K}_{i}}\left|G^{C}-Y^{C}\right|}{1+\sup _{C \in \mathcal{K}_{i}}\left|G^{C}-Y^{C}\right|}
$$

Using basic properties of measurable mappings we have that

$$
G \rightarrow \sup _{C \in \mathcal{K}_{i}}\left|G^{C}-Y^{C}\right|
$$

for any $Y \in \mathbb{R}^{d}$, is also a $\overline{\mathcal{C}}_{1}$-measurable mappings, for any $i \in \mathbb{N}_{+}$. Therefore for any $\varepsilon \in \mathbb{R}_{+}$

$$
\left\{G \in \mathbb{R}^{d}, \sup _{C \in \mathcal{K}_{i}}\left|G^{C}-Y^{C}\right| \leq \varepsilon\right\}=\bigcap_{C \in \mathcal{K}_{i}}\left\{G \in \mathbb{R}^{d},\left|G^{C}-Y^{C}\right| \leq \varepsilon\right\}
$$

which are $\overline{\mathcal{C}}_{1}$-measurable. Henceforth, (2.63) implies (2.64).

Now notice that, according to Liptser and Shiryaev (1989) we know that $\Lambda_{\mathbb{Q}^{\prime}}$ and $\Lambda_{\mathbb{Q}^{\prime \prime}}$ are dense in $\mathbb{R}_{+}$. Therefore $\Lambda_{\mathbb{Q}^{\prime}} \cap \Lambda_{\mathbb{Q}^{\prime \prime}}$ is also dense in $\mathbb{R}_{+}$, and we conclude that $\mathbb{Q}^{\prime}=\mathbb{Q}^{\prime \prime}$ because a probability in the Borelian $\sigma$-field of $D_{\left[0, T^{\star}\right]}\left(\mathbb{R}^{d}, \mathcal{K}\right)$ is entirely determined by its finite-dimensional distributions. Therefore the sequence $\left(G^{n, C}\right)_{n \geq 1}$ weakly converges in $D_{\left[0, T^{\star}\right]}\left(\mathbb{R}^{d}, \mathcal{K}\right)$ towards a certain process $\tilde{G}^{C}$ with sample paths in $D_{\left[0, T^{\star}\right]}\left(\mathbb{R}^{d}, \mathcal{K}\right)$.

In order to complete the proof, we have to show the link between $\tilde{G}^{C}$ and $G^{C}$. According to Billingsley (1999), and using the continuity property of the following mappings

$$
\Pi_{C_{1}, \ldots, C_{m}}: G \rightarrow\left(G^{C_{1}}, \ldots, G^{C_{m}}\right)
$$


from $D\left(\mathbb{R}^{d}\right)$ to $D\left(\mathbb{R}^{d}, \mathcal{K}\right)$, and

$$
\Pi_{t_{1}, \ldots, t_{k}}:\left(G^{C_{1}}, \ldots, G^{C_{m}}\right) \rightarrow\left(G_{t_{1}}^{C_{1}}, \ldots, G_{t_{1}}^{C_{m}}, \ldots, G_{t_{k}}^{C_{1}}, \ldots, G_{t_{k}}^{C_{m}}\right)
$$

from $D\left(\mathbb{R}^{d}, \mathcal{K}\right)$ to $\mathbb{R}^{d \cdot n \cdot m}$, for every $t_{1}, \ldots, t_{n} \in \bar{\Lambda}_{\mathbb{Q}^{\prime}}$ where

$$
\bar{\Lambda}_{\mathbb{Q}^{\prime}}=\left\{t>0, \mathbb{Q}^{\prime} \circ \Pi_{C_{1}, \ldots, C_{m}}^{-1}\left(G \in D_{\left[0, T^{\star}\right]}\left(\mathbb{R}^{d}, \mathcal{K}\right), \Delta G_{t}=0\right)=1\right\} \cup\{0\}
$$

and we obtain the weak convergence in $\mathbb{R}^{d \cdot k \cdot m}$ from $\mathbb{Q}^{n \prime} \circ \Pi_{C_{1}, \ldots, C_{m}}^{-1} \circ \Pi_{t_{1}, \ldots, t_{k}}^{-1}$ towards $\mathbb{Q}^{\prime} \circ \Pi_{C_{1}, \ldots, C_{m}}^{-1} \circ$ $\Pi_{t_{1}, \ldots, t_{k}}^{-1}$. We know that $\mathbb{Q}^{n \prime} \circ \Pi_{C_{1}, \ldots, C_{m}}^{-1} \circ \Pi_{t_{1}, \ldots, t_{k}}^{-1}$ is the law in $\mathbb{R}^{d \cdot k \cdot m}$ of a vector $\left(G_{t_{1}}^{C_{1}}, \ldots, G_{t_{1}}^{C_{m}}, \ldots, G_{t_{k}}^{C_{1}}, \ldots, G_{t_{k}}^{C_{m}}\right)$. Using the weak convergence of the finite-dimensional distribution we know that, for every $t_{1}, \ldots, t_{k} \in \bar{\Lambda}_{\mathbb{Q}^{\prime}}$

$$
\left(G_{t_{1}}^{n, C_{1}}, \ldots, G_{t_{1}}^{n, C_{m}}, \ldots, G_{t_{k}}^{n, C_{1}}, \ldots, G_{t_{k}}^{n, C_{m}}\right) \rightarrow\left(G_{t_{1}}^{C_{1}}, \ldots, G_{t_{1}}^{C_{m}}, \ldots, G_{t_{k}}^{C_{1}}, \ldots, G_{t_{k}}^{C_{m}}\right)
$$

or

$$
\mathbb{Q}^{\prime} \circ \Pi_{C_{1}, \ldots, C_{m}}^{-1} \circ \Pi_{t_{1}, \ldots, t_{k}}^{-1}=\mathbb{Q}_{C_{1}, \ldots, C_{m}} \circ \Pi_{t_{1}, \ldots, t_{k}}^{-1}
$$

where $\mathbb{Q}_{C_{1}, \ldots, C_{m}}$ is the law in $D\left(\mathbb{R}^{d}, \mathcal{K}\right)$ of the processes $\left(G^{C_{1}}, \ldots, G^{C_{m}}\right)$. Because a probability in $D\left(\mathbb{R}^{d}, \mathcal{K}\right)$, is entirely determined by its finite-dimensional distributions then

$$
\mathbb{Q}^{\prime} \circ \Pi_{C_{1}, \ldots, C_{m}}^{-1}=\mathbb{Q}_{C_{1}, \ldots, C_{m}}
$$

Hence we have the link between $\tilde{G}^{C}$ and $G^{C}$ : for every $m \in \mathbb{N}_{+}$and $C_{1}, \ldots, C_{m} \in \mathbb{R}_{+}$the law in $D\left(\mathbb{R}^{d}, \mathcal{K}\right)$ of the processes $\left(\tilde{G}^{C_{1}}, \ldots, \tilde{G}^{C_{m}}\right)$ and $\left(G^{C_{1}}, \ldots, G^{C_{m}}\right)$ coincide.

\subsubsection{Previsibility conditions for weak convergence of $G^{C}$ in the space $D_{\left[0, T^{\star}\right]}\left(\mathbb{R}^{d}, \mathcal{K}\right)$}

This section is devoted to develop the conditions in Theorem 95 in terms of the characteristics or triplet. Roughly speaking, the idea that we have in mind is related with the following question: Under which conditions, in terms of the triplet, our model will converge in distribution to the continuous time model that the market imposes?

Let us first define the setting assuming that we are given a "sufficiently rich" stochastic bases $\left(\Omega^{n}, \mathbb{F}^{n}, \mathbb{P}^{n}\right)$ and $(\Omega, \mathbb{F}, \mathbb{P})$ endowed with the filtrations $\mathbb{F}^{n}=\left(\mathcal{F}_{t}^{n}\right)_{t \in\left[0, T^{\star}\right]}$ and $\mathbb{F}=\left(\mathcal{F}_{t}\right)_{t \in\left[0, T^{\star}\right]}$. Let $G^{n, C}=\left(G^{n, C}(t)\right)_{C \in \mathcal{K}, t \geq 0}$ and $G^{C}=\left(G^{C}(t)\right)_{C \in \mathcal{K}, t \geq 0}$ be semimartingales depending on the process $C=\left(C_{t}\right)_{t \in\left[0, T^{\star}\right]}$ with $C \in \mathcal{K}^{(m)}$, defined on the spaces $\left(\Omega^{n}, \mathbb{F}^{n}, \mathbb{P}^{n}\right)$ and $(\Omega, \mathbb{F}, \mathbb{P})$ respectively.

Additionally, we assume that the semimartingale $G^{n, C}$ is a special ${ }^{16}$ and locally square-integrable martingale, or in other words, a martingale that admits the canonical decomposition

$$
G_{t}^{n, C}=G_{0}^{n, C}+M_{t}^{n, C}+B_{t}^{n, C}
$$

where $M_{t}^{n, C}$ is a local $\mathbb{P}$-martingale and $B_{t}^{n, C}$ is a predictable process with finite variation over each finite interval. Let us assume that $G^{C}$ is a continuous semimartingale ${ }^{17}$, that admits also the canonical

\footnotetext{
${ }^{16}$ See Liptser and Shiryayev (1986) definition 2.2

${ }^{17}$ It is well-known that interest rates derivatives quotes use the Black-76 model. This model is a continuous-time model, and it generates continuous sample-paths. However, taking into account the tenor structure, we can strip the quoted volatilities into a set of forward volatilities with skews/smile for each tenor. It basically implies that if $n=$ number of tenors, $G^{n, C}$ has to be a LIBOR additive process with jumps and $G^{C}$ is a LIBOR additive process without discontinuities.
} 
decomposition

$$
G_{t}^{C}=G_{0}^{C}+M_{t}^{C}+B_{t}^{C}
$$

This means that the characteristics of $G^{n, C}$ and $G^{C}$ are $\left(\gamma^{n, C}, A^{n, C}, v^{n, C}\right)$ and $\left(\gamma^{C}, A^{C}, v^{C}\right)$, respectively, with $v^{C}=0$ in the second case.

The aim of this section is to give conditions expressed in terms of the predictable characteristics $\left(\gamma^{n, C}, A^{n, C}, v^{n, C}\right)$ and $\left(\gamma^{C}, A^{C}, v^{C}\right)$ of the semimartingales $G^{n, C}$ and $G^{C}$, providing the existence of the modifications processes $G^{n, C}=\left(G^{C, n}(t)\right)_{C \in \mathcal{K}, t>0}$ and $G^{C}=\left(G^{C}(t)\right)_{C \in \mathcal{K}, t>0}$ with trajectories in $D_{\left[0, T^{\star}\right]}\left(\mathbb{R}^{d}, \mathcal{K}\right)$ for which a weak convergence in $D_{\left[0, T^{\star}\right]}\left(\mathbb{R}^{d}, \mathcal{K}\right)$ takes place. During the present subsection, basically we follow Liptser and Shiryaev (1989) section 8.3.

In addition to the previous definitions of $G^{n, C}$ and $G^{C}$, and for the sake of simplicity, let us introduce $\tilde{G}^{n, C}$ and $\tilde{G}^{C}$ as the continuous martingale components of $G^{n, C}$ and $G^{C}$, respectively, or in other words,

$$
\tilde{G}_{t}^{n, C}=\tilde{G}_{0}^{n, C}+\sum_{j \leq \eta(t)} \int_{T_{j}}^{t \wedge T_{j+1}} \sigma_{j}^{n}(u, C) d W_{u}^{n}
$$

and

$$
\tilde{G}_{t}^{C}=\tilde{G}_{0}^{C}+\sum_{j \leq \eta(t)} \int_{T_{j}}^{t \wedge T_{j+1}} \sigma_{j}(u, C) d W_{u}
$$

with the usual conditions on the coefficients (see Chapter 1 , or section 1 in this chapter).

Therefore, using the assumption that $G_{t}^{n, C}$ is a special semimartingale, we have

$$
G_{t}^{n, C}=G_{0}^{n, C}+\tilde{B}_{t}^{n, C}+\tilde{G}_{t}^{n, C}+\sum_{j \leq \eta(t)} \int_{T_{j}}^{t \wedge T_{j+1}} \int_{\mathbb{R}^{d}} \delta(u, x)\left(\mu_{j}^{n, C}-v_{j}^{n, C}\right)(d u, d x)
$$

where $\tilde{B}_{t}^{n, C}$ is a process, with locally integrable variation, such that

$$
\tilde{B}_{t}^{n, C}=\sum_{j \leq \eta(t)} \int_{T_{j}}^{t \wedge T_{j+1}} \alpha_{j}^{n}(u, C) d u<\infty
$$

Let us assume that for any $C, C^{\prime} \in \mathcal{K}^{(m)} \backslash\{1\}$ the previsible quadratic variation of $W_{t}^{C}$ and $W_{t}^{C^{\prime}}$ is, for every $t \geq 0$,

$$
\left\langle W^{C}, W^{C^{\prime}}\right\rangle_{t}=\sum_{j \leq \eta(t)} \int_{T_{j}}^{t \wedge T_{j+1}} \operatorname{cov}_{j}\left(u, W^{C}, W^{C^{\prime}}\right) d u
$$

Additionally, and for the sake of simplicity in the sequel, we denote with $M^{n, C}=\left(M_{t}^{n, C}\right)_{C \in \mathcal{K}, t \geq 0}$ the martingale part of the semimartingale $G^{n, C}$, for every $t \geq 0$, such that

$$
M_{t}^{n, C}=\sum_{j \leq \eta(t)} \int_{T_{j}}^{t \wedge T_{j+1}} \sigma_{j}^{n}(u, C) d W_{u}^{n}+\sum_{j \leq \eta(t)} \int_{T_{j}}^{t \wedge T_{j+1}} \int_{\mathbb{R}^{d}} \delta(u, x)\left(\mu_{j}^{n, C}-v_{j}^{n, C}\right)(d u, d x)
$$

and the quadratic variation $\left\langle M^{n, C}\right\rangle_{t}$ is given by (according to Liptser and Shiryaev (1989) Theorem 
$\left\langle M^{n, C}\right\rangle_{t}=\left\langle\tilde{G}^{n, C}\right\rangle_{t}+\sum_{j \leq \eta(t)} \int_{T_{j}}^{t \wedge T_{j+1}} \int_{\mathbb{R}^{d}} \delta^{2}(u, x) v_{j}^{n, C}(d u, d x)-\sum_{j \leq \eta(t)} \int_{T_{j}}^{t \wedge T_{j+1}} \int_{\mathbb{R}^{d}} \delta(u, x)\left(v_{j}^{n, C}(\{u\}, d x)\right)^{2}$

Let us recall that our goal is to translate the conditions of Theorem 95 in terms of the previsible characteristics of the semimartingales. Notice that assuming that $G^{n, C}$ is a special and locally square-integrable martingale, and the semimartingale $G^{C}$ is continuous with deterministic initial value, is equivalent to condition 1 in Theorem 95. Therefore, in order to obtain the weak convergence, we have to establish the following group of conditions:

Condition Group (1): in order to establish Condition (2) in Theorem 95 for every $C \in \mathcal{K}$ we need

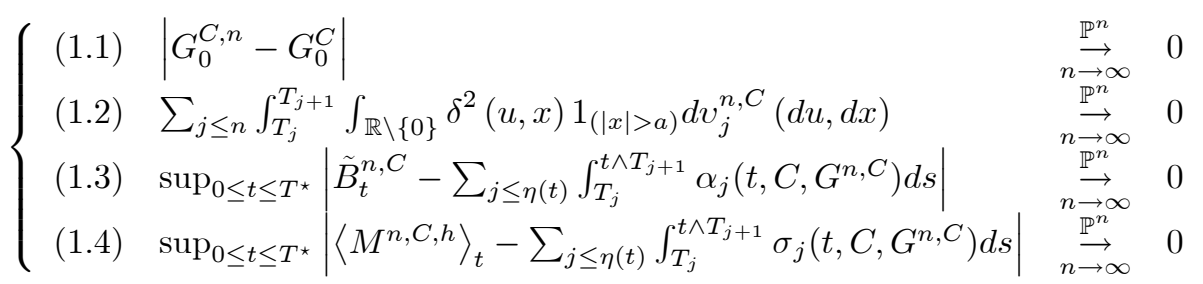

Condition Group (2): Additionally, to obtain weak convergence in the 'fidis', jointly with condition group 1, for every $C, C^{\prime} \in \mathcal{K}^{(m)} \backslash\{1\}$ we need

$$
\sup _{0<t \leq 1}\left|\left\langle\tilde{G}^{n, C}, \tilde{G}^{n, C^{\prime}}\right\rangle_{t}-\sum_{j \leq \eta(t)} \int_{T_{j}}^{t \wedge T_{j+1}} \operatorname{cov}_{j}\left(u, W^{C}, W^{C^{\prime}}\right) d u\right| \underset{n \rightarrow \infty}{\stackrel{\mathbb{P}^{n}}{\rightarrow}} 0
$$

Condition Group (3): Finally, to obtain the Condition (3) in Theorem 95, assume $p \geq 2$ and $\alpha>m$ such that for a bounded stopping times $\tau$ and $C, C^{\prime} \in \mathcal{K}^{(m)} \backslash\{1\}, C \neq C^{\prime}$ we have

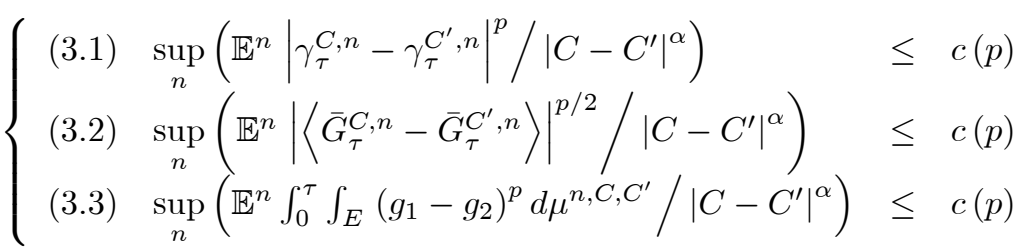

where $\tau=\inf \left\{0<t \leq 1: \sup _{\left|C-C^{\prime}\right| \leq \delta}\left|G_{t}^{C, n}-G_{t}^{C^{\prime}, n}\right| \geq \epsilon\right\}$ and $c(p)$ is a positive constant.

Theorem 96 We suppose that the condition groups (1) to (3) are satisfied. Then, the processes $G^{C, n}=$ $\left(G^{C, n}(t)\right)_{C \in \mathcal{K}, t \geq 0}$ and $G^{C}=\left(G^{C}(t)\right)_{C \in \mathcal{K}, t \geq 0}$ have paths in $D_{\left[0, T^{\star}\right]}\left(\mathbb{R}^{d}, \mathcal{K}\right)$ such that weak convergence $G^{C, n} \stackrel{w\left(\mathbb{P}^{n}\right)}{\longrightarrow} G^{C}$ in $D_{\left[0, T^{\star}\right]}\left(\mathbb{R}^{d}, \mathcal{K}\right)$ takes place.

Basically, the proof of Theorem 96 requires the verification of the conditions of Theorem 95 according to the following plan:

- First, we suppose that $G^{C, n}=\left(G^{C, n}(t)\right)_{C \in \mathcal{K}, t \geq 0}$ and $G^{C}=\left(G^{C}(t)\right)_{C \in \mathcal{K}, t \geq 0}$ are the paths in $D_{\left[0, T^{\star}\right]}\left(\mathbb{R}^{d}, \mathcal{K}\right)$. Using the conditions of group 1 we establish a weak convergence of the process $G^{C, n}$ to $G^{C}$ with $C \in \mathcal{K}^{(m)}$, given $m \in \mathbb{N}_{+}$, in $D_{\left[0, T^{\star}\right]}\left(\mathbb{R}^{d}, \mathcal{K}\right)$. In turn, this convergence implies the condition 2) in Theorem 95. 
- Second, using the conditions of group 1 and 2 we establish the convergence of the finite-dimensional distributions

$$
\left(G^{n, C_{1}}, G^{n, C_{2}}, \ldots, G^{n, C_{m}}\right) \underset{n \rightarrow \infty}{\stackrel{\mathbb{P}^{n}}{\rightarrow}}\left(G^{C_{1}}, G^{C_{2}}, \ldots, G^{C_{m}}\right)
$$

in the Skorohod space $D_{\left[0, T^{\star}\right]}\left(\mathbb{R}^{d}, \mathcal{K}\right)$ for every $m \geq 2$.

- Third, using the conditions of group 3, the result from Valkelia and Dzaparidze (1990), we prove that for every $C, C^{\prime} \in \mathcal{K}^{(m)} \backslash\{1\}$ and a bounded stopping time $\tau$ :

$$
\sup _{n} \mathbb{E}^{n}\left(\left|G_{\tau}^{C, n}-G_{\tau}^{C^{\prime}, n}\right|^{p} /\left|C-C^{\prime}\right|^{\alpha}\right) \leq \bar{C}_{i}
$$

and using specially chosen stopping times, this inequality and the lemma about the estimation of the modulus of continuity allow us to verify the condition 2) of Theorem 95.

In order to prove the Theorem 96, we need the following results.

Proposition 97 Let $G^{C}$ be a stochastic process defined on the stochastic basis $(\Omega, \mathcal{F}, \mathbb{P})$ with sample-paths in the Skorohod space $D_{\left[0, T^{\star}\right]}\left(\mathbb{R}^{d}, \mathcal{K}\right)$. Then for any $\varepsilon>0$ the function $\tau$ defined in $\Omega$ by

$$
\tau(\omega)=\inf \left(t>0, \sup _{\substack{C^{\prime}, C \in \mathcal{K}_{\delta}^{(m)} \\\left|C^{\prime}-C\right| \leq \delta}}\left|G_{t}^{C^{\prime}}(\omega)-G_{t}^{C}(\omega)\right| \geq \varepsilon\right) \wedge T^{\star}
$$

is a $\mathcal{F}$-stopping time.

Proof. According to Lipster and Shiryaev (1989) let us define the process $Z$ as

$$
Z_{t}(\omega)=\sup _{\substack{C^{\prime}, C \in \mathcal{K}_{\delta}^{(m)} \\\left|C^{\prime}-C\right| \leq \delta}}\left|G_{t}^{C^{\prime}}(\omega)-G_{t}^{C}(\omega)\right|
$$

and notice that it is enough to prove that $Z$ is a process with sample-paths in $D_{\left[0, T^{\star}\right]}\left(\mathbb{R}^{d}, \mathcal{K}\right), \mathcal{F}_{t^{-}}$adapted. Let $\omega \in \Omega$. Then we have for every $s, t \geq 0$

$$
\begin{aligned}
\left|Z_{s}(\omega)-Z_{t}(\omega)\right| & =\sup _{\substack{C^{\prime}, C \in \mathcal{K}_{\delta}^{(m)} \\
\left|C^{\prime}-C\right| \leq \delta}}\left|G_{s}^{C^{\prime}}(\omega)-G_{s}^{C}(\omega)\right|-\sup _{\substack{C^{\prime}, C \in \mathcal{K}_{\delta}^{(m)} \\
\left|C^{\prime}-C\right| \leq \delta}}\left|G_{t}^{C^{\prime}}(\omega)-G_{t}^{C}(\omega)\right| \\
\leq & \sup _{\substack{C^{\prime}, C \in \mathcal{K}_{\delta}^{(m)} \\
\left|C^{\prime}-C\right| \leq \delta}}\left|G_{s}^{C^{\prime}}(\omega)-G_{s}^{C}(\omega)-G_{t}^{C^{\prime}}(\omega)+G_{t}^{C}(\omega)\right| \\
\leq & 2 \sup _{C^{\prime}, C \in \mathcal{K}_{\delta}^{(m)}}\left|G_{s}^{C}(\omega)-G_{t}^{C}(\omega)\right|
\end{aligned}
$$

Notice that $G^{C}$ has sample-paths in $D_{\left[0, T^{\star}\right]}\left(\mathbb{R}^{d}, \mathcal{K}\right)$. Therefore we have that

$$
\lim _{\delta \rightarrow 0} \inf _{\substack{\Pi_{\delta}^{(n)} \\ t_{j+1}-t_{j}>\delta}} \max _{0 \leq j \leq n} \sup _{s, t \in\left[t_{j}, t_{j+1}\right)}\left|Z_{s}(\omega)-Z_{t}(\omega)\right|=0
$$

This, according to Lemma 77 , proves that $Z$ is a process with sample-paths in $D_{\left[0, T^{\star}\right]}\left(\mathbb{R}^{d}, \mathcal{K}\right)$.

In order to prove that $Z$ is a $\mathcal{F}_{t}$-adapted process, notice that for any $\omega \in \Omega$, and any $t \in \mathbb{R}_{+}$, the 
mapping $C \rightarrow G_{t}^{C}(\omega)$ from $\mathcal{K}$ to $\mathbb{R}^{d}$ is continuous. Therefore we have that, for every $t \in \mathbb{R}_{+}$and any $C \in \mathcal{K}^{(m)}, Z$ is a $\mathcal{F}_{t^{-} \text {adapted process. }}$

Proposition 98 The condition 3 ) in Theorem 95 is equivalent to

$$
\lim _{\delta \rightarrow 0} \sup _{n \geq 1} \mathbb{P}_{n}\left(\sup _{\substack{C^{\prime}, C \in \mathcal{K}_{\delta}^{(m)} \\\left|C^{\prime}-C\right| \leq \delta}}\left|G_{\tau}^{n, C^{\prime}}(\omega)-G_{\tau}^{n, C}(\omega)\right| \geq \varepsilon\right)=0
$$

where $\tau$ is the $\mathcal{F}$-stopping time defined in Proposition 97.

Proof. Let us recall that according to Proposition 97, the stopping time $\tau(\omega)$ was defined as

$$
\tau(\omega)=\inf \left(t>0, \sup _{\substack{C^{\prime}, C \in \mathcal{K}_{\delta}^{(m)} \\\left|C^{\prime}-C\right| \leq \delta}}\left|G_{t}^{n, C^{\prime}}(\omega)-G_{t}^{n, C}(\omega)\right| \geq \varepsilon\right) \wedge T^{\star}
$$

Hence

$$
\mathbb{P}_{n}\left(\mathbb{L L}_{\delta}^{(m)}\left(G^{n}\right)>\varepsilon\right)=\mathbb{P}_{n}\left(\sup _{0 \leq t \leq T^{\star}} \sup _{\substack{C^{\prime}, C \in \mathcal{K}_{\delta}^{(m)} \\\left|C^{\prime}-C\right| \leq \delta}}\left|G_{t}^{n, C^{\prime}}-G_{t}^{n, C}\right|>\varepsilon\right) \leq \mathbb{P}_{n}\left(\sup _{\substack{C^{\prime}, C \in \mathcal{K}_{\delta}^{(m)} \\\left|C^{\prime}-C\right| \leq \delta}}\left|G_{\tau}^{n, C^{\prime}}-G_{\tau}^{n, C}\right| \geq \varepsilon\right)
$$

Conversely, it is easy to see

$$
\begin{aligned}
& \mathbb{P}_{n}\left(\sup _{\substack{C^{\prime}, C \in \mathcal{K}_{\delta}^{(m)} \\
\left|C^{\prime}-C\right| \leq \delta}}\left|G_{\tau}^{n, C^{\prime}}-G_{\tau}^{n, C}\right| \geq \varepsilon\right) \leq \mathbb{P}_{n}\left(\mathbb{L L}_{\delta}^{(m)}\left(G^{n}\right) \geq \varepsilon\right) \\
& \quad
\end{aligned}
$$

Another relevant result that we need to prove Theorem 96, is the next one, given by Dzhaparidze and Valkeila (1990)

Lemma 99 Let $M$ be a locally square integrable martingale with $M_{0}=0$. Let $T$ be a stopping time. If $v$ is the compensator of the jump measure of $M$, then there exist for every $p \geq 2$ constants $k_{p}$ and $K_{p}$ such that

$$
\begin{aligned}
k_{p} \mathbb{E}\left(\langle M\rangle_{T}^{p / 2}+\int_{0}^{T} \int_{\mathbb{R} \backslash\{0\}}|g|^{p} d v\right) & \leq \mathbb{E}\left(\sup _{0 \leq t \leq T}\left|M_{t}\right|^{p}\right) \\
& \leq K_{p} \mathbb{E}\left(\langle M\rangle_{T}^{p / 2}+\int_{0}^{T} \int_{\mathbb{R} \backslash\{0\}}|g|^{p} d v\right)
\end{aligned}
$$

Proof. cf. Dzhaparidze and Valkeila (1990) Lemma 2.1 p.108, based on the Burkholder-Gundy inequality for martingales.

And the last useful result in our proof is the following,

Proposition 100 Let $\tau$ be a finite stopping time and $C_{1}, \ldots, C_{m} \in \mathcal{K}$ and $m \in \mathbb{N}_{+}$. Additionally let define 
$\mu^{n}$ the integer-valued random measure for jumps of a process $\left(G^{n, C_{1}}, \ldots, G^{n, C_{m}}\right)$ and $v^{n}$ the compensator, let $f$ be a real function on $\mathbb{R}$ such that the following integrals has sense for any $i=1, \ldots, m$

1) $\int_{0}^{t} \int_{\mathbb{R} \backslash\{0\}} f\left(g_{i}\right) \mu^{n}\left(d t, d g_{1}, \ldots, d g_{i}, \ldots, d g_{m}\right)=\int_{0}^{\tau} \int_{\mathbb{R} \backslash\{0\}} f(g) d \mu^{n, C_{i}}$

2) $\int_{0}^{t} \int_{\mathbb{R} \backslash\{0\}} f\left(g_{i}\right)\left(\mu^{n}-v^{n}\right)\left(d t, d g_{1}, \ldots, d g_{i}, \ldots, d g_{m}\right)=\int_{0}^{\tau} \int_{\mathbb{R} \backslash\{0\}} f(g) d\left(\mu^{n, C_{i}}-v^{n, C_{i}}\right)$

Proof. Both results can be proved using similar ways. Let us define two local martingales $M=\left(M_{t}\right)_{t \geq 0}$ and $M^{\prime}=\left(M_{t}^{\prime}\right)_{t \geq 0}$ for any $t \in \mathbb{R}^{+}$,

$$
M_{t}=\int_{0}^{t} \int_{\mathbb{R} \backslash\{0\}} f\left(g_{i}\right)\left(\mu^{n, C_{i}}-v^{n, C_{i}}\right)\left(d t, d g_{1}, \ldots, d g_{i}, \ldots, d g_{m}\right)
$$

and

$$
M_{t}^{\prime}=\int_{0}^{t} \int_{\mathbb{R} \backslash\{0\}} f(g) d\left(\mu^{n, C_{i}}-v^{n, C_{i}}\right)
$$

Notice that $M$ and $M^{\prime}$ are purely discontinuous processes and to prove that they are indistinguishable, it is enough to show for any $t \in \mathbb{R}^{+}$

$$
\Delta M_{t}=\Delta M_{t}^{\prime}
$$

or

$$
\Delta M_{t}=\int_{\mathbb{R} \backslash\{0\}} f\left(g_{i}\right)\left(\mu^{n}\left(\{t\}, d g_{1}, \ldots, d g_{i}, \ldots, d g_{m}\right)-v^{n}\left(\{t\}, d g_{1}, \ldots, d g_{i}, \ldots, d g_{m}\right)\right)
$$

and

$$
\Delta M_{t}^{\prime}=\int_{\mathbb{R} \backslash\{0\}} f(g) d\left(\mu^{n, C_{i}}(\{t\}, d g)-v^{n, C_{i}}(\{t\}, d g)\right)
$$

Notice that the processes

$$
\left(\int_{\mathbb{R} \backslash\{0\}} f\left(g_{i}\right)\left(v^{n}\left(\{t\}, d g_{1}, \ldots, d g_{i}, \ldots, d g_{m}\right)\right)\right)_{t \geq 0} \quad \text { and } \quad\left(\int_{\mathbb{R} \backslash\{0\}} f(g)\left(v^{n, C_{i}}(\{t\}, d g)\right)\right)_{t \geq 0}
$$

are respectively the compensators of

$$
\left(\int_{\mathbb{R} \backslash\{0\}} f\left(g_{i}\right)\left(\mu^{n}\left(\{t\}, d g_{1}, \ldots, d g_{i}, \ldots, d g_{m}\right)\right)\right)_{t \geq 0} \quad \text { and } \quad\left(\int_{\mathbb{R} \backslash\{0\}} f(g)\left(\mu^{n, C_{i}}(\{t\}, d g)\right)\right)_{t \geq 0}
$$

and it is enough to establish the following equality for any $t \in \mathbb{R}^{+}$:

$$
\int_{\mathbb{R} \backslash\{0\}} f\left(g_{i}\right)\left(\mu^{n}\left(\{t\}, d g_{1}, \ldots, d g_{i}, \ldots, d g_{m}\right)\right)=\int_{\mathbb{R} \backslash\{0\}} f(g)\left(\mu^{n, C_{i}}(\{t\}, d g)\right)
$$

and in order to complete the proof we only need to see that

$$
\int_{\mathbb{R} \backslash\{0\}} f\left(g_{i}\right)\left(\mu^{n}\left(\{t\}, d g_{1}, \ldots, d g_{i}, \ldots, d g_{m}\right)\right)=f\left(\Delta G_{t}^{n, C_{i}}\right)
$$

and

$$
\int_{\mathbb{R} \backslash\{0\}} f(g)\left(\mu^{n, C_{i}}(\{t\}, d g)\right)=f\left(\Delta G_{t}^{n, C_{i}}\right)
$$

Finally, we can proceed with the proof of Theorem 96.

Proof. (Theorem 96) Let us proceed with the first part. To prove weak convergence condition according with Theorem 95 or equivalently, to prove the weak convergence from $G^{C, n}$ to $G^{C}$ with $C \in$ 
$\mathcal{K}^{(m)}$, given $m \geq \mathbb{N}_{+}$, in $D_{\left[0, T^{\star}\right]}\left(\mathbb{R}^{d}, \mathcal{K}\right)$, means, according to Lipster and Shiryaev (1989) Theorem 8.3.1 (p.625), the following conditions, for every $C \in \mathcal{K}^{(m)}$, given $m \geq \mathbb{N}_{+}$, for every $a \in(0,1]$ and $0<t \leq T^{\star} \leq 1$ :

$$
\begin{aligned}
& \left.1^{\prime}\right)\left|G_{0}^{C, n}-G_{0}^{C}\right| \stackrel{\mathbb{P}^{n}}{\rightarrow} 0 \\
& \left.2^{\prime}\right) \sum_{j \leq n} \int_{T_{j}}^{T_{j+1}} \int_{\mathbb{R} \backslash\{0\}} 1_{(|x|>a)} d v_{j}^{n, C} \stackrel{\mathbb{P}^{n}}{\rightarrow} 0 \\
& \left.3^{\prime}\right) \sup _{0 \leq t \leq T^{\star}}\left|B_{t}^{n, C}-\sum_{j \leq \eta(t)} \int_{T_{j}}^{t \wedge T_{j+1}} \alpha_{\eta(s)}\left(t, C, G^{n, C}\right) d s\right| \stackrel{\mathbb{P}^{n}}{\rightarrow} 0 \\
& \left.4^{\prime}\right) \sup _{0 \leq t \leq T^{\star}}\left|\left\langle M^{n, C, h}\right\rangle_{t}-\sum_{j \leq \eta(t)} \int_{T_{j}}^{t \wedge T_{j+1}} \sigma_{\eta(s)}\left(t, C, G^{n, C}\right) d s\right| \stackrel{\mathbb{P}^{n}}{\rightarrow} 0
\end{aligned}
$$

Now, we have to prove that conditions $1.1,1.2,1.3$ and 1.4 (in the condition group 1 ) imply $1^{\prime}, 2^{\prime}, 3^{\prime}$ and $4^{\prime}$. It is clear that 1.1 implies $1^{\prime}$. The condition $2^{\prime}$ can be proved using 1.2 and the inequality, for any $\varepsilon>0$,

$$
\mathbb{P}^{n}\left(\sum_{j \leq n} \int_{T_{j}}^{T_{j+1}} \int_{\mathbb{R} \backslash\{0\}} 1_{(|x|>h)} d v_{j}^{n, C} \geq \varepsilon\right) \leq \mathbb{P}^{n}\left(\sum_{j \leq n} \int_{T_{j}}^{T_{j+1}} \int_{\mathbb{R} \backslash\{0\}}^{2} \delta^{2} 1_{(|x|>h)} d v_{j}^{n, C} \geq \varepsilon a^{2}\right)
$$

Related with the condition $3^{\prime}$, we use the canonical decomposition for semimartingales and special semimartingales (using directly definitions in Liptser and Shiryaev (1989) 4.1.1 and Jacod and Shiryaev (1999) $I I .2 .38)$

$$
\begin{aligned}
B_{t}^{n, C}= & G_{t}^{n, C}-G_{0}^{n, C}-\tilde{G}_{t}^{n, C}-\sum_{j \leq \eta(t)} \int_{T_{j}}^{t \wedge T_{j+1}} \int_{\mathbb{R}^{d}} \delta(u, x) 1_{\{|x| \leq 1\}}\left(\mu_{j}^{n, C}-v_{j}^{n, C}\right)(d u, d x) \\
& -\sum_{j \leq \eta(t)} \int_{T_{j}}^{t^{(n)} \wedge T_{j+1}} \int_{\mathbb{R}^{d}} \delta(u, x) 1_{\{|x|>1\}} \mu_{j}^{n, C}(d u, d x)
\end{aligned}
$$

and

$$
\tilde{B}_{t}^{n, C}=G_{t}^{n, C}-G_{0}^{n, C}-\tilde{G}_{t}^{n, C}-\sum_{j \leq \eta(t)} \int_{T_{j}}^{t \wedge T_{j+1}} \int_{\mathbb{R}^{d}} \delta(u, x)\left(\mu_{j}^{n, C}-v_{j}^{n, C}\right)(d u, d x)
$$

Therefore

$$
B_{t}^{n, C}-\tilde{B}_{t}^{n, C}=-\sum_{j \leq \eta(t)} \int_{T_{j}}^{t \wedge T_{j+1}} \int_{\mathbb{R}^{d}} \delta(u, x) 1_{\{|x|>1\}} v_{j}^{n, C}(d u, d x)
$$

and we obtain

$$
\begin{aligned}
\mathbb{P}^{n}\left(\sup _{0 \leq t \leq T^{\star}}\left|B_{t}^{n, C}-\tilde{B}_{t}^{n, C}\right| \geq \varepsilon\right) & \leq \mathbb{P}^{n}\left(\sum_{j \leq n} \int_{T_{j}}^{T_{j+1}} \int_{\mathbb{R}^{d}}|\delta(u, x)| 1_{\{|x|>1\}} v_{j}^{n, C}(d u, d x) \geq \varepsilon\right) \\
& \leq \mathbb{P}^{n}\left(\sum_{j \leq n} \int_{T_{j}}^{T_{j+1}} \int_{\mathbb{R}^{d}}(\delta(u, x))^{2} 1_{\{|x|>1\}} v_{j}^{n, C}(d u, d x) \geq \varepsilon\right)
\end{aligned}
$$

This shows that conditions 1.3 and $3^{\prime}$ are equivalent, using condition 1.2. 
Related with condition $4^{\prime}$, let us recall

$$
\begin{aligned}
\left\langle M^{n, C, h}\right\rangle_{t}= & \left\langle\tilde{G}^{n, C}\right\rangle_{t}+\sum_{j \leq \eta(t)} \int_{T_{j}}^{t \wedge T_{j+1}} \int_{\mathbb{R}^{d}} \delta^{2}(u, x) 1_{\{|x| \leq h\}} v_{j}^{n, C}(d u, d x) \\
& -\sum_{j \leq \eta(t)} \sum_{T_{j} \leq u \leq t \wedge T_{j+1}} \int_{\mathbb{R}^{d}} \delta(u, x) 1_{\{|x| \leq h\}}\left(v_{j}^{n, C}(\{u\}, d x)\right)^{2}
\end{aligned}
$$

and

$$
\begin{aligned}
\left\langle M^{n, C}\right\rangle_{t}= & \left\langle\tilde{G}^{n, C}\right\rangle_{t}+\sum_{j \leq \eta(t)} \int_{T_{j}}^{t \wedge T_{j+1}} \int_{\mathbb{R}^{d}} \delta^{2}(u, x) v_{j}^{n, C}(d u, d x) \\
& -\sum_{j \leq \eta(t)} \sum_{T_{j} \leq u \leq t \wedge T_{j+1}} \int_{\mathbb{R}^{d}} \delta(u, x)\left(v_{j}^{n, C}(\{u\}, d x)\right)^{2}
\end{aligned}
$$

Let us simplify the notation redefining $\delta(u, x)=\delta$. Therefore

$$
\begin{aligned}
\left|\left\langle M^{n, C, h}\right\rangle_{t}-\left\langle M^{n, C}\right\rangle_{t}\right| \leq & \sum_{j \leq \eta(t)} \int_{T_{j}}^{t \wedge T_{j+1}} \int_{\mathbb{R}^{d}} \delta^{2} 1_{\{|x|>h\}} v_{j}^{n, C}(d u, d x) \\
& +\sum_{j \leq \eta(t)} \sum_{T_{j} \leq u \leq t \wedge T_{j+1}} \mid \int_{\mathbb{R}^{d}} \delta 1_{\{|x| \leq h\}}\left(v_{j}^{n, C}(\{u\}, d x)\right)^{2} \\
& -\int_{\mathbb{R}^{d}} \delta\left(v_{j}^{n, C}(\{u\}, d x)\right)^{2} \mid \\
\leq & \sum_{j \leq \eta(t)} \int_{T_{j}}^{t \wedge T_{j+1}} \int_{\mathbb{R}^{d}} \delta^{2} 1_{\{|x|>h\}} v_{j}^{n, C}(d u, d x)+\sum_{j \leq \eta(t)} \sum_{T_{j} \leq u \leq t \wedge T_{j+1}} \int_{\mathbb{R}^{d}}|\delta| 1_{\{|x|>h\}} v_{j}^{n, C}(\{u\}, d x) \\
& \times\left(\int_{\mathbb{R}^{d}}|\delta| 1_{\{|x| \leq h\}} v_{j}^{n, C}(\{u\}, d x)+\int_{\mathbb{R}^{d}}|\delta| v_{j}^{n, C}(\{u\}, d x)\right) \\
= & \sum_{j \leq \eta(t)} \int_{T_{j}}^{t \wedge T_{j+1}} \int_{\mathbb{R}^{d}} \delta^{2} 1_{\{|x|>h\}} v_{j}^{n, C}(d u, d x)+\sum_{j \leq \eta(t)} \sum_{T_{j} \leq u \leq t \wedge T_{j+1}} \int_{\mathbb{R}^{d}}|\delta| 1_{\{|x|>h\}} v_{j}^{n, C}(\{u\}, d x) \\
& \times\left(2 \int_{\mathbb{R}^{d}}|\delta| 1_{\{|x| \leq h\}} v_{j}^{n, C}(\{u\}, d x)+\int_{\mathbb{R}^{d}}|\delta| 1_{\{|x|>h\}} v_{j}^{n, C}(\{u\}, d x)\right)
\end{aligned}
$$

taking into account that, using Liptser and Shiryaev (1989), for any $t \geq 0$ and any $\omega \in \Omega$

$$
v_{j}^{n, C}(\omega,\{t\} \times \mathbb{R}) \leq 1
$$


we have

$$
\begin{aligned}
\left|\left\langle M^{n, C, h}\right\rangle_{t}-\left\langle M^{n, C}\right\rangle_{t}\right| \leq & \sum_{j \leq \eta(t)} \int_{T_{j}}^{t \wedge T_{j+1}} \int_{\mathbb{R}^{d} \backslash\{0\}} \delta^{2} 1_{\{|x|>h\}} v_{j}^{n, C}(d u, d x) \\
& +\sum_{j \leq \eta(t)} \sum_{T_{j} \leq u \leq t \wedge T_{j+1}} 2 a \int_{\mathbb{R}^{d}}|\delta| 1_{\{|x|>h\}} v_{j}^{n, C}(\{u\}, d x) \\
& +\sum_{j \leq \eta(t)} \sum_{T_{j} \leq u \leq t \wedge T_{j+1}}\left(\int_{\mathbb{R}^{d}}|\delta| 1_{\{|x|>h\}} v_{j}^{n, C}(\{u\}, d x)\right)^{2} \\
\leq & \sum_{j \leq \eta(t)} \int_{T_{j}}^{t \wedge T_{j+1}} \int_{\mathbb{R}^{d}} \delta^{2} 1_{\{|x|>h\}} v_{j}^{n, C}(d u, d x) \\
& +2 a \sum_{j \leq \eta(t)} \int_{T_{j}}^{T_{j+1}} \int_{\mathbb{R}^{d}}|\delta| 1_{\{|x|>h\}} d v_{j}^{n, C} \\
& +\sum_{j \leq \eta(t)} \sum_{T_{j} \leq u \leq t \wedge T_{j+1}} \int_{\mathbb{R}^{d}} \delta^{2} 1_{\{|x|>h\}} v_{j}^{n, C}(\{u\}, d x)
\end{aligned}
$$

and finally, we obtain the inequality

$$
\sup _{0 \leq t \leq T^{\star}}\left|\left\langle M^{n, C, h}\right\rangle_{t}-\left\langle M^{n, C}\right\rangle_{t}\right| \leq 4 \sum_{j \leq \eta(t)} \int_{T_{j}}^{T_{j+1}} \int_{\mathbb{R}^{d}} \delta^{2} 1_{\{|x|>h\}} d v_{j}^{n, C}
$$

This means that conditions 1.4 and $4^{\prime}$ are equivalent.

Therefore, all the conditions of Theorem 8.2.1 in Liptser and Shiryaev (1989) pp.608 - 609 are satisfied and we have

$$
G^{C, n} \stackrel{\mathbb{P}^{n}}{\rightarrow} G^{C}
$$

for every $C \in \mathcal{K}^{(m)}$, given $m \geq \mathbb{N}_{+}$, in the Skorohod space $D_{\left[0, T^{\star}\right]}\left(\mathbb{R}^{d}, \mathcal{K}\right)$.

Second, we have to prove the convergence of the finite-dimensional distributions

$$
\left(G^{n, C_{1}}, G^{n, C_{2}}, \ldots, G^{n, C_{m}}\right) \underset{n \rightarrow \infty}{\stackrel{\mathbb{P}^{n}}{\rightarrow}}\left(G^{C_{1}}, G^{C_{2}}, \ldots, G^{C_{m}}\right)
$$

in the Skorohod space $D_{\left[0, T^{\star}\right]}\left(\mathbb{R}^{d}, \mathcal{K}\right)$ for every $m \geq 2$.

Let us define $Y^{n}=\left(G^{n, C_{1}}, G^{n, C_{2}}, \ldots, G^{n, C_{m}}\right)$ and $Y=\left(G^{C_{1}}, G^{C_{2}}, \ldots, G^{C_{m}}\right)$. Then, it is enough to prove the weak convergence of $Y^{n}$ to $Y$ in $D_{\left[0, T^{\star}\right]}\left(\mathbb{R}^{d}, \mathcal{K}\right)$. Additionally, let us define

$$
\bar{B}_{\eta(t)}(Y)=\left(\alpha_{\eta(t)}\left(t, C_{1}, G^{C_{1}}\right), \ldots, \alpha_{\eta(t)}\left(t, C_{m}, G^{C_{m}}\right)\right)
$$

and

$$
\bar{C}_{\eta(t)}(Y)=\left(\begin{array}{cccc}
\sigma_{\eta(t)}\left(t, C_{1}, G^{C_{1}}\right) & 0 & \cdots & 0 \\
0 & \ddots & \ddots & \vdots \\
\vdots & \ddots & \ddots & 0 \\
0 & \cdots & 0 & \sigma_{\eta(t)}\left(t, C_{m}, G^{C_{m}}\right)
\end{array}\right)
$$

such that

$$
Y_{t}=Y_{0}+\sum_{j \leq \eta(t)} \int_{T_{j}}^{t \wedge T_{j+1}} \bar{B}_{\eta(s)}(Y) d s+\sum_{j \leq \eta(t)} \int_{T_{j}}^{t \wedge T_{j+1}} \bar{C}_{\eta(t)}(Y) d\left(W^{C_{1}}, \ldots, W^{C_{m}}\right)
$$


According to Liptser and Shiryaev (1989) section 8.3.5 there exists a Brownian motion in $\mathbb{R}^{m}\left(\tilde{W}^{1}, \ldots, \tilde{W}^{m}\right)$ such that the process $Y$ satisfies the following stochastic differential equation

$$
Y_{t}=Y_{0}+\sum_{j \leq \eta(t)} \int_{T_{j}}^{t \wedge T_{j+1}} \bar{B}_{\eta(s)}(Y) d s+\sum_{j \leq \eta(t)} \int_{T_{j}}^{t \wedge T_{j+1}} \bar{D}_{\eta(t)}(Y)^{1 / 2} d\left(\tilde{W}^{1}, \ldots, \tilde{W}^{m}\right)
$$

where $\bar{D}_{\eta(t)}(Y)^{1 / 2}$ is the square root of the semidefinite-positive matrix $\bar{D}_{\eta(t)}(Y)=\left(\bar{D}_{\eta(t)}(Y)(i, j)\right)_{1 \leq i, j \leq m}$ given by

$$
\bar{D}_{\eta(t)}(Y)(i, j)=\sigma_{\eta(t)}\left(t, C_{i}, G^{C_{i}}\right) \sigma_{\eta(t)}\left(t, C_{j}, G^{C_{j}}\right) \operatorname{cov}\left(G_{1}^{C_{i}}, G_{1}^{C_{j}}\right)
$$

On the other hand, let us define $\mu^{n}$ as the jump-measure of $Y^{n}$ and let $v^{n}$ be the compensator. Then $\mu^{n}$ and $v^{n}$ are random measures on $\mathbb{R}^{+} \times\left(\mathbb{R}^{m} \backslash\{0\}\right)$ and we can choose a version of $v^{n}$ such that for every $t \in \mathbb{R}^{+}$

$$
v^{n}\left(\{t\} \times\left(\mathbb{R}^{m} \backslash\{0\}\right)\right) \leq 1
$$

Additionally let us denote as $\mathcal{B}_{t}^{n}=\left(B_{t}^{n, C_{1}}, \ldots, B_{t}^{n, C_{m}}\right)$, and $\mathcal{C}_{t}^{n}$ as the $m \times m$ matrix of predictable quadratic variation at $t \in \mathbb{R}^{+}$. This basically represents the continuous martingale part of $Y^{n}$ such that for any $h>0$

$$
\begin{aligned}
\mathcal{M}_{t}^{n, h}= & \mathcal{C}_{t}^{n}+\sum_{j \leq \eta(t)} \int_{T_{j}}^{t \wedge T_{j+1}} \int_{\mathbb{R}^{m} \backslash\{0\}} \delta \delta^{\top} 1_{\{\|x\| \leq h\}} v_{j}^{n, C}(d u, d x) \\
& -\sum_{j \leq \eta(t)} \sum_{T_{j} \leq u \leq t \wedge T_{j+1}} \int_{\mathbb{R}^{m} \backslash\{0\}} \delta 1_{\{\|x\| \leq h\}} v_{j}^{n, C}(\{u\}, d x) \times \int_{\mathbb{R}^{m} \backslash\{0\}} \delta 1_{\{\|x\| \leq h\}} v_{j}^{n, C}(\{u\}, d x)
\end{aligned}
$$

where \|\| is the Euclidean norm in $\mathbb{R}^{m}$. Therefore, for any $i=1, \ldots, m$ and any $j=1, \ldots, m$ we denote $\mathcal{M}_{t}^{n, h}(i, j)$ as the $(i, j)$-th value in the matrix $\mathcal{M}_{t}^{n, h}$.

Let us recall the conditions established in Theorem 8.3.3 in Liptser and Shiryaev (1989) in order to prove the weak convergence of $\left(Y^{n}\right)_{n \geq 1}$ to $Y$, for any $h \in(0,1]$, any $t \leq T \in \mathbb{R}_{+}$and any $i, j=1, \ldots, m$ :

$\left.1^{\prime \prime}\right)\left|Y_{0}^{n}-Y_{0}\right| \stackrel{\mathbb{P}^{n}}{\rightarrow} 0$

$$
\begin{aligned}
& \left.2^{\prime \prime}\right) \sum_{j \leq n} \int_{T_{j}}^{T_{j+1}} \int_{\mathbb{R} \backslash\{0\}} 1_{(\|x\|>h)} d v_{j}^{n} \stackrel{\mathbb{P}^{n}}{\rightarrow} 0 \\
& \left.3^{\prime \prime}\right) \sup _{0 \leq t \leq T^{\star}}\left\|\mathcal{B}_{t}^{n}-\sum_{j \leq \eta(t)} \int_{T_{j}}^{t \wedge T_{j+1}} \bar{B}_{\eta(s)}(Y) d s\right\| \stackrel{\mathbb{P}^{n}}{\rightarrow} 0 \\
& \left.4^{\prime \prime}\right) \sup _{0 \leq t \leq T^{\star}}\left|\mathcal{M}_{t}^{n, h}(i, j)-\sum_{j \leq \eta(t)} \int_{T_{j}}^{t \wedge T_{j+1}} \bar{D}_{\eta(s)}(Y)(i, j) d s\right| \stackrel{\mathbb{P}^{n}}{\rightarrow} 0
\end{aligned}
$$

In turn, according to the Cramer-Wold theorem (in Billingsley (1986) p.397), $1^{\prime \prime}$ ) it is equivalent to prove for any $\left(\lambda_{1}, \ldots, \lambda_{m}\right) \in \mathbb{R}^{m}$ that

$$
\sum_{i \leq m} \lambda_{i} G_{0}^{n, C_{i}} \stackrel{\mathbb{P}^{n}}{\rightarrow} \sum_{i \leq m} \lambda_{i} G_{0}^{C_{i}}
$$

which is equivalent to the condition 1 of group 1 (condition 1.1). 
As to $\left.2^{\prime \prime}\right)$, noting that for any $h>0$

$$
\left\{x=\left(x_{1}, x_{2}, \ldots, x_{m}\right):\|x\|>h\right\} \subset \bigcup_{q \leq m}\left\{x=\left(x_{1}, x_{2}, \ldots, x_{m}\right): x_{q}^{2}>\frac{h^{2}}{m}\right\}
$$

we have

$$
\begin{aligned}
\sum_{j \leq n} \int_{T_{j}}^{T_{j+1}} \int_{\mathbb{R} \backslash\{0\}} 1_{(\|x\|>h)} d v_{j}^{n} & \leq \sum_{j \leq n} \int_{T_{j}}^{T_{j+1}} \int_{\mathbb{R} \backslash\{0\}} 1\left(\left\{x_{1}^{2}>\frac{h^{2}}{m}\right\} \cup \ldots \cup\left\{x_{m}^{2}>\frac{h^{2}}{m}\right\}\right) \\
& =\sum_{q \leq m} \sum_{j \leq n} \int_{T_{j}}^{T_{j+1}} \int_{\mathbb{R} \backslash\{0\}} 1\left(\left\{x_{q}^{2}>\frac{h^{2}}{m}\right\}\right) d v_{j}^{n}
\end{aligned}
$$

Therefore, according to Proposition 100

$$
\sum_{j \leq n} \int_{T_{j}}^{T_{j+1}} \int_{\mathbb{R} \backslash\{0\}} 1_{(\|x\|>h)} d v_{j}^{n} \leq \sum_{q \leq m} \sum_{j \leq n} \int_{T_{j}}^{T_{j+1}} \int_{\mathbb{R} \backslash\{0\}} 1_{\left(\left\{\left|x_{q}\right|>\frac{h^{2}}{m}\right\}\right)} d v_{j}^{n, C_{q}}
$$

This proves that $2^{\prime \prime}$ ) is given by the hypotheses 2 in the group of conditions 1 (condition 1.2).

To check the condition $\left.3^{\prime \prime}\right)$ let us recall the inequality $(a+b)^{1 / 2} \leq a^{1 / 2}+b^{1 / 2}$ for every $a, b>0$ and taking into account this result, it is easy to see

$$
\sup _{0 \leq t \leq T^{\star}}\left\|\mathcal{B}_{t}^{n}-\sum_{j \leq \eta(t)} \int_{T_{j}}^{t \wedge T_{j+1}} \bar{B}_{\eta(s)}(Y) d s\right\| \leq \sum_{q \leq m} \sup _{0 \leq t \leq T^{\star}}\left|B_{t}^{n, C_{q}}-\sum_{j \leq \eta(t)} \int_{T_{j}}^{t \wedge T_{j+1}} \alpha_{\eta(s)}\left(t, C_{q}, G^{n, C_{q}}\right) d s\right|
$$

Finally notice that the condition 3 in group 1 (condition 1.3) implies the equivalent condition $3^{\prime}$ ) and consequently $\left.3^{\prime \prime}\right)$.

Before proving condition $4^{\prime \prime}$ ) we have to check the following result: for any $h>0$

$$
\sum_{j \leq n} \int_{T_{j}}^{T_{j+1}} \int_{\mathbb{R} \backslash\{0\}}\|x\|^{2} 1_{(\|x\|>h)} d v_{j}^{n} \stackrel{\mathbb{P}^{n}}{\rightarrow} 0
$$

Notice that we have

$$
\begin{aligned}
& \sum_{j \leq n} \int_{T_{j}}^{T_{j+1}} \int_{\mathbb{R} \backslash\{0\}}\|x\|^{2} 1_{(\|x\|>h)} d v_{j}^{n}=\sum_{q \leq m} \sum_{j \leq n} \int_{T_{j}}^{T_{j+1}} \int_{\mathbb{R} \backslash\{0\}} x_{q}^{2} 1_{\left(\left\{\left|x_{q}\right|>h\right\}\right)} d v_{j}^{n} \\
= & \sum_{q \leq m}\left(\sum_{j \leq n} \int_{T_{j}}^{T_{j+1}} \int_{\mathbb{R} \backslash\{0\}} x_{q}^{2} 1_{\left(\{\|x\|>h\} \cap\left\{\left|x_{q}\right|>h\right\}\right)} d v_{j}^{n}+\sum_{j \leq n} \int_{T_{j}}^{T_{j+1}} \int_{\mathbb{R} \backslash\{0\}} x_{q}^{2} 1_{\left(\{\|x\|>h\} \cap\left\{\left|x_{q}\right| \leq h\right\}\right)} d v_{j}^{n}\right) \\
\leq & \sum_{q \leq m} \sum_{j \leq n} \int_{T_{j}}^{T_{j+1}} \int_{\mathbb{R} \backslash\{0\}} x_{q}^{2} 1_{\left(\left\{\left|x_{q}\right|>h\right\}\right)} d v_{j}^{n}+a^{2} \sum_{q \leq m} \sum_{j \leq n} \int_{T_{j}}^{T_{j+1}} \int_{\mathbb{R} \backslash\{0\}} 1_{(\{\|x\|>h\})} d v_{j}^{n} \\
\leq & \sum_{q \leq m} \sum_{j \leq n} \int_{T_{j}}^{T_{j+1}} \int_{\mathbb{R} \backslash\{0\}} x_{q}^{2} 1_{\left(\left\{\left|x_{q}\right|>h\right\}\right)} d v_{j}^{n, C_{q}}+a^{2} m \sum_{j \leq n} \int_{T_{j}}^{T_{j+1}} \int_{\mathbb{R} \backslash\{0\}} 1_{(\{\|x\|>h\})} d v_{j}^{n}
\end{aligned}
$$


We have proved that condition 1.2 implies condition $2^{\prime \prime}$ ) and therefore

$$
a^{2} m \sum_{j \leq n} \int_{T_{j}}^{T_{j+1}} \int_{\mathbb{R} \backslash\{0\}} 1_{(\{\|x\|>h\})} d v_{j}^{n} \stackrel{\mathbb{P}^{n}}{\rightarrow} 0
$$

On the other hand, using condition also 1.2 we have that for every $q=1, \ldots, m$

$$
\sum_{j \leq n} \int_{T_{j}}^{T_{j+1}} \int_{\mathbb{R} \backslash\{0\}} x_{q}^{2} 1_{\left(\left\{\left|x_{q}\right|>h\right\}\right)} d v_{j}^{n, C_{q}} \stackrel{\mathbb{P}^{n}}{\rightarrow} 0
$$

This shows that (2.66) is true.

The next step is to prove condition $4^{\prime \prime}$ ) using (2.66). According to expression (2.65), for any $p, q \in$ $\{1, \ldots, m\}$ we have

$$
\begin{aligned}
& \sup _{0 \leq t \leq T^{\star}} \mid\left\langle\tilde{G}^{n, C_{p}}, \tilde{G}^{n, C_{q}}\right\rangle_{t}+\sum_{j \leq \eta(t)} \int_{T_{j}}^{t \wedge T_{j+1}} \int_{\mathbb{R}^{m} \backslash\{0\}} x_{p} x_{q} 1_{\{\|x\| \leq h\}} v_{j}^{n}(d u, d x) \\
& -\sum_{j \leq \eta(t)} \sum_{T_{j} \leq u \leq t \wedge T_{j+1}} \int_{\mathbb{R}^{m} \backslash\{0\}} x_{p} 1_{\{\|x\| \leq h\}} v_{j}^{n, C}(\{u\}, d x) \times \int_{\mathbb{R}^{m} \backslash\{0\}} x_{q} 1_{\{\|x\| \leq h\}} v_{j}^{n, C}(\{u\}, d x) \\
& -\sum_{j \leq \eta(t)} \int_{T_{j}}^{t \wedge T_{j+1}} \sigma_{\eta(s)}\left(t, C_{p}, G^{C_{p}}\right) \sigma_{\eta(s)}\left(t, C_{q}, G^{C_{q}}\right) \operatorname{cov}\left(W_{1}^{C_{p}}, W_{1}^{C_{q}}\right) d s \mid \stackrel{\mathbb{P}^{n}}{\rightarrow} 0
\end{aligned}
$$

Notice that

$$
\operatorname{cov}\left(W_{1}^{C_{p}}, W_{1}^{C_{q}}\right) s=\left\langle W^{C_{p}}, W^{C_{q}}\right\rangle_{s}
$$

Taking into account the condition group (2), it is enough to prove

$$
\begin{aligned}
& \sup _{0 \leq t \leq T^{\star}} \mid\left\langle M^{n, C_{p}}, M^{n, C_{q}}\right\rangle_{t}-\left\langle\tilde{G}^{n, C_{p}}, \tilde{G}^{n, C_{q}}\right\rangle_{t}+\sum_{j \leq \eta(t)} \int_{T_{j}}^{t \wedge T_{j+1}} \int_{\mathbb{R}^{m} \backslash\{0\}} x_{p} x_{q} 1_{\{\|x\| \leq h\}} v_{j}^{n}(d u, d x) \\
& +\sum_{j \leq \eta(t)} \sum_{T_{j} \leq u \leq t \wedge T_{j+1}} \int_{\mathbb{R}^{m} \backslash\{0\}} x_{p} 1_{\{\|x\| \leq h\}} v_{j}^{n, C}(\{u\}, d x) \times \int_{\mathbb{R}^{m} \backslash\{0\}} x_{q} 1_{\{\|x\| \leq h\}} v_{j}^{n, C}(\{u\}, d x) \mid \stackrel{\mathbb{P}^{n}}{\rightarrow} 0
\end{aligned}
$$

For this note that

$$
\begin{aligned}
\sup _{0 \leq t \leq T^{\star}} & \mid\left\langle M^{n, C_{p}}, M^{n, C_{q}}\right\rangle_{t}-\left\langle\tilde{G}^{n, C_{p}}, \tilde{G}^{n, C_{q}}\right\rangle_{t}+\sum_{j \leq \eta(t)} \int_{T_{j}}^{t \wedge T_{j+1}} \int_{\mathbb{R}^{m} \backslash\{0\}} x_{p} x_{q} 1_{\{\|x\| \leq h\}} v_{j}^{n}(d u, d x) \\
& +\sum_{j \leq \eta(t)} \sum_{T_{j} \leq u \leq t \wedge T_{j+1}} \int_{\mathbb{R}^{m} \backslash\{0\}} x_{p} 1_{\{\|x\| \leq h\}} v_{j}^{n}(\{u\}, d x) \times \int_{\mathbb{R}^{m} \backslash\{0\}} x_{q} 1_{\{\|x\| \leq h\}} v_{j}^{n}(\{u\}, d x) \mid
\end{aligned}
$$




$$
\begin{aligned}
\leq & \sup _{0 \leq t \leq T^{\star}} \mid \sum_{j \leq \eta(t)} \int_{T_{j}}^{t \wedge T_{j+1}} \int_{\mathbb{R}^{m} \backslash\{0\}} x_{p} x_{q} 1_{\{\|x\| \leq h\}} v_{j}^{n}(d u, d x)-\sum_{j \leq \eta(t)} \int_{T_{j}}^{t \wedge T_{j+1}} \int_{\mathbb{R}^{m} \backslash\{0\}} x_{p} x_{q} v_{j}^{n}(d u, d x) \\
& +\sum_{j \leq \eta(t)} \sum_{T_{j} \leq u \leq t \wedge T_{j+1}} \int_{\mathbb{R}^{m} \backslash\{0\}} x_{p} 1_{\{\|x\| \leq h\}} v_{j}^{n}(\{u\}, d x) \times \int_{\mathbb{R}^{m} \backslash\{0\}} x_{q} 1_{\{\|x\| \leq h\}} v_{j}^{n}(\{u\}, d x) \\
& -\sum_{j \leq \eta(t)} \sum_{T_{j} \leq u \leq t \wedge T_{j+1}} \int_{\mathbb{R}^{m} \backslash\{0\}} x_{p} v_{j}^{n}(\{u\}, d x) \times \int_{\mathbb{R}^{m} \backslash\{0\}} x_{q} v_{j}^{n}(\{u\}, d x) \mid \\
\leq & \sum_{j \leq n} \int_{T_{j}}^{T_{j+1}} \int_{\mathbb{R}^{m} \backslash\{0\}}\left|x_{p} x_{q}\right| 1_{\{\|x\|>h\}} v_{j}^{n}(d u, d x)+\sum_{j \leq n} \sum_{T_{j} \leq u \leq T_{j+1}} \int_{\mathbb{R}^{m} \backslash\{0\}}\left|x_{p}\right| 1_{\{\|x\| \leq h\}} v_{j}^{n}(\{u\}, d x) \\
& \times\left[\left|\int_{\mathbb{R}^{m} \backslash\{0\}} x_{q} 1_{\{\|x\| \leq h\}} v_{j}^{n}(\{u\}, d x)-\int_{\mathbb{R}^{m} \backslash\{0\}} x_{q} v_{j}^{n}(\{u\}, d x)\right|\right] \\
& +\sum_{j \leq n} \sum_{T_{j} \leq u \leq T_{j+1}} \int_{\mathbb{R}^{m} \backslash\{0\}}\left|x_{q}\right| 1_{\{\|x\| \leq h\}} v_{j}^{n}(\{u\}, d x) \\
& \times\left[\left|\int_{\mathbb{R}^{m} \backslash\{0\}} x_{p} 1_{\{\|x\| \leq h\}} v_{j}^{n}(\{u\}, d x)-\int_{\mathbb{R}^{m} \backslash\{0\}} x_{p} v_{j}^{n}(\{u\}, d x)\right|\right] \\
& +\sum_{j \leq n} \sum_{T_{j} \leq u \leq T_{j+1}} \int_{\mathbb{R}^{m} \backslash\{0\}}\left|x_{q}\right| 1_{\{\|x\|>h\}} v_{j}^{n}(\{u\}, d x) \\
& \times\left[\left|\int_{\mathbb{R}^{m} \backslash\{0\}} x_{p} 1_{\{\|x\| \leq h\}} v_{j}^{n}(\{u\}, d x)-\int_{\mathbb{R}^{m} \backslash\{0\}} x_{i} v_{j}^{n}(\{u\}, d x)\right|\right]
\end{aligned}
$$

Notice that we have assumed that $v^{n}\left(\{t\} \times\left(\mathbb{R}^{m} \backslash\{0\}\right)\right) \leq 1$. Therefore

$$
\begin{aligned}
& \quad \sup _{0 \leq t \leq T^{*}} \mid\left\langle M^{n, C_{i}}, M^{n, C_{j}}\right\rangle_{t}-\left\langle\tilde{G}^{n, C_{i}}, \tilde{G}^{n, C_{j}}\right\rangle_{t}+\sum_{j \leq \eta(t)} \int_{T_{j}}^{t \wedge T_{j+1}} \int_{\mathbb{R}^{m} \backslash\{0\}} x_{p} x_{j} 1_{\{\|x\| \leq h\}} v_{j}^{n}(d u, d x) \\
& \quad+\sum_{j \leq \eta(t)} \sum_{T_{j} \leq u \leq t \wedge T_{j+1}} \int_{\mathbb{R}^{m} \backslash\{0\}} x_{p} 1_{\{\|x\| \leq h\}} v_{j}^{n}(\{u\}, d x) \times \int_{\mathbb{R}^{m} \backslash\{0\}} x_{j} 1_{\{\|x\| \leq h\}} v_{j}^{n}(\{u\}, d x) \mid \\
& \leq \sum_{j \leq n} \int_{T_{j}}^{T_{j+1}} \int_{\mathbb{R}^{m} \backslash\{0\}}\left|x_{p} x_{j}\right| 1_{\{\|x\|>h\}} v_{j}^{n}(d u, d x)+a \sum_{j \leq n} \sum_{T_{j} \leq u \leq T_{j+1}} \int_{\mathbb{R}^{m} \backslash\{0\}}\left|x_{j}\right| 1_{\{\|x\| \leq h\}} v_{j}^{n}(\{u\}, d x) \\
& \quad+a \sum_{j \leq n} \sum_{T_{j} \leq u \leq T_{j+1}} \int_{\mathbb{R}^{m} \backslash\{0\}}\left|x_{p}\right| 1_{\{\|x\|>h\}} v_{j}^{n}(\{u\}, d x) \\
& +\sum_{j \leq n} \sum_{T_{j} \leq u \leq T_{j+1}} \int_{\mathbb{R}^{m} \backslash\{0\}}\left|x_{p}\right| 1_{\{\|x\|>h\}} v_{j}^{n}(\{u\}, d x) \int_{\mathbb{R}^{m} \backslash\{0\}}\left|x_{q}\right| 1_{\{\|x\|>h\}} v_{j}^{n}(\{u\}, d x)
\end{aligned}
$$


Using the inequality $a b \leq \frac{1}{2}\left(a^{2}+b^{2}\right)$ valid for every $a, b>0$, we have

$$
\begin{aligned}
& \sup _{0 \leq t \leq T^{*}} \mid\left\langle M^{n, C_{p}}, M^{n, C_{q}}\right\rangle_{t}-\left\langle\tilde{G}^{n, C_{p}}, \tilde{G}^{n, C_{q}}\right\rangle_{t}+\sum_{j \leq \eta(t)} \int_{T_{j}}^{t \wedge T_{j+1}} \int_{\mathbb{R}^{m} \backslash\{0\}} x_{p} x_{q} 1_{\{\|x\| \leq h\}} v_{j}^{n}(d u, d x) \\
+ & \sum_{j \leq \eta(t)} \sum_{T_{j} \leq u \leq t \wedge T_{j+1}} \int_{\mathbb{R}^{m} \backslash\{0\}} x_{p} 1_{\{\|x\| \leq h\}} v_{j}^{n}(\{u\}, d x) \times \int_{\mathbb{R}^{m} \backslash\{0\}} x_{q} 1_{\{\|x\| \leq h\}} v_{j}^{n}(\{u\}, d x) \mid \\
\leq & \frac{1}{2} \sum_{j \leq n} \int_{T_{j}}^{T_{j+1}} \int_{\mathbb{R}^{m} \backslash\{0\}} x_{p}^{2} 1_{\{\|x\|>h\}} v_{j}^{n}(d u, d x)+\frac{1}{2} \sum_{j \leq n} \int_{T_{j}}^{T_{j+1}} \int_{\mathbb{R}^{m} \backslash\{0\}} x_{q}^{2} 1_{\{\|x\|>h\}} v_{j}^{n}(d u, d x) \\
& +a \sum_{j \leq n} \int_{T_{j}}^{T_{j+1}} \int_{\mathbb{R}^{m} \backslash\{0\}}\left|x_{p}\right| 1_{\{\|x\|>h\}} v_{j}^{n}(d u, d x)+a \sum_{j \leq n} \int_{T_{j}}^{T_{j+1}} \int_{\mathbb{R}^{m} \backslash\{0\}}\left|x_{q}\right| 1_{\{\|x\|>h\}} v_{j}^{n}(d u, d x) \\
& +\frac{1}{2} \sum_{j \leq n} \int_{T_{j}}^{T_{j+1}} \int_{\mathbb{R}^{m} \backslash\{0\}} x_{p}^{2} 1_{\{\|x\|>h\}} v_{j}^{n}(d u, d x)+\frac{1}{2} \sum_{j \leq n} \int_{T_{j}}^{T_{j+1}} \int_{\mathbb{R}^{m} \backslash\{0\}} x_{q}^{2} 1_{\{\|x\|>h\}} v_{j}^{n}(d u, d x) \\
\leq & 2 \sum_{j \leq n} \int_{T_{j}}^{T_{j+1}} \int_{\mathbb{R}^{m} \backslash\{0\}}\|x\|^{2} 1_{\{\|x\|>h\}} v_{j}^{n}(d u, d x)+2 \sum_{j \leq n} \int_{T_{j}}^{T_{j+1}} \int_{\mathbb{R}^{m} \backslash\{0\}} a\|x\| 1_{\{\|x\|>h\}} v_{j}^{n}(d u, d x) \\
\leq & 4 \sum_{j \leq n} \int_{T_{j}}^{T_{j+1}} \int_{\mathbb{R}^{m} \backslash\{0\}}\|x\|^{2} 1_{\{\|x\|>h\}} v_{j}^{n}(d u, d x)
\end{aligned}
$$

and using the expression (2.66) we achieve the condition $\left.4^{\prime \prime}\right)$.

Finally, in order to complete the proof of Theorem 96 we just need to show that the conditions of group 3) imply then condition 2) in Theorem 95. Notice that $G^{n, C^{\prime}}$ and $G^{n, C}$ are special semimartingales. Therefore

$$
G_{\tau}^{n, C}=G_{0}^{n, C}+\tilde{B}_{\tau}^{n, C}+M_{\tau}^{n, C}
$$

and

$$
G_{\tau}^{n, C^{\prime}}=G_{0}^{n, C^{\prime}}+\tilde{B}_{\tau}^{n, C^{\prime}}+M_{\tau}^{n, C^{\prime}}
$$

whence

$$
\left|G_{\tau}^{n, C}-G_{\tau}^{n, C^{\prime}}\right| \leq\left|G_{0}^{n, C}-G_{0}^{n, C^{\prime}}\right|+\left|\tilde{B}_{\tau}^{n, C}-\tilde{B}_{\tau}^{n, C^{\prime}}\right|+\left|M_{\tau}^{n, C}-M_{\tau}^{n, C^{\prime}}\right|
$$

Let us recall the following inequality, for every $a>0, b>0$ and $p \geq 1$ we have

$$
(a+b)^{p} \leq(p+1)\left(a^{p}+b^{p}\right)
$$

Therefore,

$$
\begin{aligned}
\mathbb{E}^{n}\left(\left|G_{\tau}^{n, C}-G_{\tau}^{n, C^{\prime}}\right|^{p}\right) \leq & (p+1)^{2}\left[\mathbb{E}^{n}\left(\left|G_{0}^{n, C}-G_{0}^{n, C^{\prime}}\right|^{p}\right)+\mathbb{E}^{n}\left(\left|\tilde{B}_{\tau}^{n, C}-\tilde{B}_{\tau}^{n, C^{\prime}}\right|^{p}\right)\right. \\
& \left.+\mathbb{E}^{n}\left(\left|M_{\tau}^{n, C}-M_{\tau}^{n, C^{\prime}}\right|^{p}\right)\right]
\end{aligned}
$$

Notice that if $G^{n, C^{\prime}}$ and $G^{n, C}$ are special semimartingales (locally square integrable), then the local martingales $M^{n, C^{\prime}}$ and $M^{n, C}$ are square integrable. Additionally, let us define $\tilde{\mu}^{n, C, C^{\prime}}$ as the jump measure of $M^{n, C}-M^{n, C^{\prime}}$ and $\tilde{v}^{n, C, C^{\prime}}$ its compensator. Therefore we can use directly Lemma 99 to prove 
the following inequality for any $p \geq 2$

$$
\mathbb{E}^{n}\left(\left|M_{\tau}^{n, C}-M_{\tau}^{n, C^{\prime}}\right|^{p}\right) \leq c_{p} \mathbb{E}^{n}\left(\left\langle M^{n, C}-M^{n, C^{\prime}}\right\rangle_{\tau}^{p / 2}+\sum_{j \leq \eta(t)} \int_{T_{j}}^{t \wedge T_{j+1}} \int_{\mathbb{R}^{m} \backslash\{0\}}|x|^{p} d \tilde{v}^{n, C, C^{\prime}}\right)
$$

and using Proposition 100 we have

$$
\begin{aligned}
M^{n, C}-M^{n, C^{\prime}}= & G^{n, C}-G^{n, C^{\prime}}+\sum_{j \leq \eta(t)} \int_{T_{j}}^{\cdot \wedge T_{j+1}} \int_{\mathbb{R} \backslash\{0\}} x d\left(\mu_{j}^{n, C}-v_{j}^{n, C}\right) \\
& -\sum_{j \leq \eta(t)} \int_{T_{j}}^{\cdot \wedge T_{j+1}} \int_{\mathbb{R} \backslash\{0\}} y d\left(\mu_{j}^{n, C^{\prime}}-v_{j}^{n, C^{\prime}}\right) \\
= & G^{n, C}-G^{n, C^{\prime}}+\sum_{j \leq \eta(t)} \int_{T_{j}}^{\cdot \wedge T_{j+1}} \int_{\mathbb{R}^{2} \backslash\{0,0\}}(x-y) d\left(\mu_{j}^{n, C, C^{\prime}}-v_{j}^{n, C, C^{\prime}}\right)
\end{aligned}
$$

According to Liptser and Shiryaev (1989) Theorem 3.5.1 we get

$$
\begin{aligned}
\left\langle M^{n, C}-M^{n, C^{\prime}}\right\rangle_{\tau}= & \left\langle G^{n, C}-G^{n, C^{\prime}}\right\rangle+\sum_{j \leq \eta(\tau)} \int_{T_{j}}^{\tau \wedge T_{j+1}} \int_{\mathbb{R}^{2} \backslash\{0,0\}}(x-y)^{2} d\left(v_{j}^{n, C, C^{\prime}}\right) \\
& -\sum_{j \leq \eta(\tau)} \int_{T_{j}}^{\tau \wedge T_{j+1}} \int_{\mathbb{R}^{2} \backslash\{0,0\}}(x-y) v_{j}^{n, C, C^{\prime}}(\{u\}, d x, d y)^{2}
\end{aligned}
$$

and again, using the fact that $v^{n}\left(\{t\} \times\left(\mathbb{R}^{m} \backslash\{0\}\right)\right) \leq 1$ we have

$$
\begin{aligned}
\left\langle M^{n, C}-M^{n, C^{\prime}}\right\rangle_{\tau}= & \left\langle G^{n, C}-G^{n, C^{\prime}}\right\rangle_{\tau}+\sum_{j \leq \eta(\tau)} \int_{T_{j}}^{\tau \wedge T_{j+1}} \int_{\mathbb{R}^{2} \backslash\{0,0\}}(x-y)^{2} d\left(v_{j}^{n, C, C^{\prime}}\right) \\
& +\sum_{j \leq \eta(t)} \sum_{T_{j} \leq u \leq \tau \wedge T_{j+1}} \int_{\mathbb{R}^{2} \backslash\{0,0\}}(x-y)^{2} v_{j}^{n, C, C^{\prime}}(\{u\}, d x, d y)^{2} \\
\leq & \left\langle G^{n, C}-G^{n, C^{\prime}}\right\rangle_{\tau}+\sum_{j \leq \eta(\tau)} \int_{T_{j}}^{\tau \wedge T_{j+1}} \int_{\mathbb{R}^{2} \backslash\{0,0\}}(x-y)^{2} d\left(v_{j}^{n, C, C^{\prime}}\right)
\end{aligned}
$$

Recall the inequality $(a+b)^{p} \leq(p+1)\left(a^{p}+b^{p}\right)$, for every $a>0, b>0$ and $p \geq 1$, and similarly as before

$$
\begin{aligned}
\mathbb{E}^{n}\left(\left\langle M^{n, C}-M^{n, C^{\prime}}\right\rangle_{\tau}^{p / 2}\right) \leq & \left(\frac{p}{2}+1\right)\left[\mathbb{E}^{n}\left(\left\langle G^{n, C}-G^{n, C^{\prime}}\right\rangle_{\tau}^{p / 2}\right)\right. \\
& +2^{p / 2} \mathbb{E}^{n}\left(\sum_{j \leq \eta(\tau)} \int_{T_{j}}^{\tau \wedge T_{j+1}} \int_{\mathbb{R}^{2} \backslash\{0,0\}}(x-y)^{2} d\left(v_{j}^{n, C, C^{\prime}}\right)\right)
\end{aligned}
$$

On the other hand, we have

$$
\begin{aligned}
\mathbb{E}^{n}\left(\sum_{j \leq \eta(\tau)} \int_{T_{j}}^{\tau \wedge T_{j+1}} \int_{\mathbb{R}^{2} \backslash\{0,0\}}|x|^{p} d\left(\tilde{v}_{j}^{n, C, C^{\prime}}\right)\right) & =\mathbb{E}^{n}\left(\sum_{j \leq \eta(\tau)} \int_{T_{j}}^{\tau \wedge T_{j+1}} \int_{\mathbb{R}^{2} \backslash\{0,0\}}|x|^{p} d\left(\tilde{\mu}_{j}^{n, C, C^{\prime}}\right)\right) \\
& =\mathbb{E}^{n}\left(\sum_{j \leq \eta(\tau)} \int_{T_{j}}^{\tau \wedge T_{j+1}}\left|\Delta M_{s}^{n, C}-\Delta M_{s}^{n, C^{\prime}}\right|^{p}\right)
\end{aligned}
$$


so that

$$
\begin{aligned}
& \mathbb{E}^{n}\left(\sum_{j \leq \eta(\tau)} \int_{T_{j}}^{\tau \wedge T_{j+1}}\left|\Delta M_{s}^{n, C}-\Delta M_{s}^{n, C^{\prime}}\right|^{p}\right) \\
= & \mathbb{E}^{n}\left(\sum_{j \leq \eta(\tau)} \sum_{T_{j} \leq u \leq \tau \wedge T_{j+1}} \mid \int_{\mathbb{R} \backslash\{0\}} x \mu_{j}^{n, C}(\{u\}, d x)-\int_{\mathbb{R} \backslash\{0\}} x v_{j}^{n, C}(\{u\}, d x)\right. \\
& \left.-\int_{\mathbb{R} \backslash\{0\}} y \mu_{j}^{n, C^{\prime}}(\{u\}, d x)+\left.\int_{\mathbb{R} \backslash\{0\}} y v_{j}^{n, C^{\prime}}(\{u\}, d x)\right|^{p}\right) \\
= & \mathbb{E}^{n}\left(\sum_{j \leq \eta(\tau)} \sum_{T_{j} \leq u \leq \tau \wedge T_{j+1}} \mid \int_{\mathbb{R}^{2} \backslash\{0,0\}}(x-y) \mu_{j}^{n, C, C^{\prime}}(\{u\}, d x, d y)\right. \\
& \left.-\left.\int_{\mathbb{R}^{2} \backslash\{0,0\}}(x-y) v_{j}^{n, C, C^{\prime}}(\{u\}, d x, d y)\right|^{p}\right) \\
\leq & (p+1)\left[\mathbb{E}^{n}\left(\sum_{j \leq \eta(\tau)} \sum_{T_{j} \leq u \leq \tau \wedge T_{j+1}} \int_{\mathbb{R}^{2} \backslash\{0,0\}}|x-y|_{j}^{p} \mu^{n, C, C^{\prime}}(\{u\}, d x, d y)\right)\right. \\
& \left.-\mathbb{E}^{n}\left(\sum_{j \leq \eta(\tau)} \sum_{T_{j} \leq u \leq \tau \wedge T_{j+1}} \int_{\mathbb{R}^{2} \backslash\{0,0\}}|x-y|^{p} v_{j}^{n, C, C^{\prime}}(\{u\}, d x, d y)\right)\right] \\
\leq & 2(p+1) \mathbb{E}^{n}\left(\sum_{j \leq \eta(\tau)} \int_{T_{j}}^{\tau \wedge T_{j+1}} \int_{\mathbb{R}^{2} \backslash\{0,0\}}|x-y|^{p} d v_{j}^{n, C, C^{\prime}}\right)
\end{aligned}
$$

Finally, insert the results (2.70), (2.69) and (2.68) in (2.67). We obtain for every $p \geq 2$ the existence of a constant $c_{p}$, such that

$$
\begin{aligned}
\mathbb{E}^{n}\left(\left|G_{\tau}^{n, C}-G_{\tau}^{n, C^{\prime}}\right|^{p}\right) \leq & c_{p}\left[\mathbb{E}^{n}\left(\left|G_{0}^{n, C}-G_{0}^{n, C^{\prime}}\right|^{p}\right)+\mathbb{E}^{n}\left(\left|\tilde{B}_{\tau}^{n, C}-\tilde{B}_{\tau}^{n, C^{\prime}}\right|^{p}\right)\right. \\
& +\mathbb{E}^{n}\left(\left\langle G^{n, C}-G^{n, C^{\prime}}\right\rangle_{\tau}^{p / 2}\right) \\
& \left.+\mathbb{E}^{n}\left(\left(\sum_{j \leq \eta(\tau)} \int_{T_{j}}^{\tau \wedge T_{j+1}} \int_{\mathbb{R}^{2} \backslash\{0,0\}}(x-y)^{2} d v_{j}^{n, C, C^{\prime}}\right)\right)^{p / 2}\right) \\
& \left.+\mathbb{E}^{n}\left(\sum_{j \leq \eta(\tau)} \int_{T_{j}}^{\tau \wedge T_{j+1}} \int_{\mathbb{R}^{2} \backslash\{0,0\}}|x-y|^{p} d v_{j}^{n, C, C^{\prime}}\right)\right]
\end{aligned}
$$

Now, the proof is complete, because it is easy to see that condition 2) of Theorem 95 follows immediately from the group of conditions 3 in Theorem 96 and Lemma 99. 
Chapter 3

\section{LIBOR Market Model driven by LIBOR additive processes: Pricing and Calibration}




\subsection{Preliminaries}

\subsubsection{Introduction}

As was mentioned in the previous chapters, the acronym LIBOR stands for the London Interbank Offered Rate. It is the rate of interest offered by banks on deposits from other banks in Eurocurrency markets. But also it is the floating rate commonly used in interest rate swap agreements in international financial markets. LIBOR is determined by trading between banks and changes continuously as economic conditions change.

In this Third Chapter ${ }^{1}$ we consider the most popular family of interest rate model: the LIBOR Market Model extended with jumps and credit risk. But why are such models so popular among practitioners? The main reason lies in the agreement between such models and well-established market formulas for caps/floors and swaptions, the basic interest rates derivatives.

Indeed, the lognormal forward-LIBOR model (LFM) prices caps with Black's cap formula, which is the standard formula employed in the cap market. Moreover, the lognormal forward-swap model (LSM) prices swaptions with Black's swaption formula, which again is the standard formula employed in the swaption market. Since the caps and swaptions markets are the two main markets in the interest rate options world, it is important for a model to be compatible with such market formulas. Before marketmodels were introduced, there was no interest-rate dynamics compatible with either Black's formula for caps/floors or swaptions. The introduction of market models provided a new derivation of Black's formulas based on rigorous interest-rate dynamics.

Recall that the Heath, Jarrow and Morton approach (also known as HJM) of term structure modelling (extended in Chapter II) is based on the arbitrage-free dynamics of instantaneous continuously compounded forward rates. However the assumption that instantaneous rates exist is not always convenient, since it requires a certain degree of smoothness with respect to the tenor (i.e. maturity) of bond prices and their volatilities. An alternative construction of an arbitrage-free family of bond prices, making no reference to the instantaneous, continuously compounded rates, is in some circumstances more suitable.

The basic log-normal Forward LIBOR (also known as LIBOR Market model, or BGM) model has proved to be an essential tool for pricing and risk-managing interest rate derivatives. It was the first model that was easy to calibrate to the grid of at-the-money swaptions volatilities across all swaption expiries and underlying swaps' maturities. This calibration ability is recognized as perhaps the most important requirement for a model to be successfully applied to such complicated interest rate derivatives as flexicaps, chooser caps, Bermuda swaptions, and various callable LIBOR exotics.

While the log-normal forward LIBOR model has established a standard for incorporating all available at-the-money volatility information, it was less successful in recovering other essential characteristics of interest rate markets, particularly the volatility smile. In a lognormal forward LIBOR model, swaptions of the same expiry/maturity but of different strikes have the same implied Black volatilities, a feature of the model that is inconsistent with the market. Various extensions of the log-normal forward LIBOR model have been proposed. These extensions have been designed to incorporate the volatility smile effect in one form or another. Local volatility, jump-diffusion, and stochastic volatility extensions of a forward LIBOR model have all been introduced.

\footnotetext{
${ }^{1}$ This work has been developed under the direction of Javier Nogales and Winfried Stute. I am extremely grateful to them. I also benefited greatly in my work from discussions with seminar participants at Statistical Lab. at University of Cambridge (Oct.2006), participants at IV Bachelier Congress in Tokyo (2006), and very specially with Rama Cont, David Nualart, C. Rogers, J.H. León, W. Runggaldier and T. Schmidt. Their advice, suggestions and assistance has been key in the development of this work. Special thanks to the Fixed Income Derivatives desk of Caja Madrid, for the set of historical market volatilities provided. Comments and suggestions are welcome, all errors are my own.

Contact email: jpcolino@gmail.com
} 
The importance of recovering all volatility smiles for all swaptions cannot be underestimated. Structured interest rate derivatives, derivatives that are a natural application for forward LIBOR market models because of their complicated volatility dependence, are rarely, if ever, depend on at-the-money volatilities. They are typically structured with strikes that are far away from money, and typically depend on swaption volatilities with different expiries and maturities. To accurately value such an instrument, a model should be able to match all market volatilities for all strikes for many, if not all, swaption expiry/maturity combinations. As market sophistication grows and bid/ask spreads on structured derivatives tighten, such requirements become more and more imperative.

A model that can calibrate to market-implied skews for the whole swaption grid is needed. This third Chapter introduces such a model, a model that aims to reproduce the skew structure modeling what a log-normal forward LIBOR model did for the volatility structure modeling. We build a model that takes a swaption grid of volatility skews as an external input. By allowing the Lévy parameters responsible for inducing skews on swaptions to be time-dependent and LIBOR rate specific, we are able to create a model that can recover all available market volatility smile information.

Therefore, in this third chapter, two results are presented:

- First, a new framework to model forward LIBOR interest rates with credit risk, and the no-arbitrage conditions under risk-neutral or forward-neutral probability.

- Second, we investigate new methodologies of calibration for LIBOR market model with jumps, that adjust not only the usual at-the-money swaption prices but also include the information from in/out-of-the-money swaptions. It is based in convex programming that guarantee global solutions and stability in the calibration against market changes.

This third chapter is structured as follows:

- Section 1 is basically devoted to introduce the preliminaries and basics such that definitions, notation and previous models that will be basic to understand the subsequent sections. We introduce and summarize the main ideas about the log-normal LIBOR market model, the Lévy Market model and the Lévy forward price model. Additionally, we develop the LIBOR market model driven by a LIBOR additive process and we obtain the non-arbitrage conditions under the risk-neutral measure.

- Probably, Section 2 is the most practical and numerical section where we propose a calibration methodology to fit this sort of models to the swaption market prices, not only at-the-money swaptions but also in/out-of-the-money swaptions.

\subsubsection{Basic assumption for the LIBOR additive model with credit transitions}

In this section, we mainly focus on forward LIBOR rates assuming, first, that the dynamics of defaultable instantaneous forward rates are specified through the Heath, Jarrow and Morton (1992) model, driven by LIBOR additive processes (piecewise homogeneous Lévy process) as in Chapter 2. And second, we additionally assume that the LIBOR rates can be derived from the bond prices of forward prices. In order to achieve this aim, we have to establish some assumptions that will be applied during the whole work.

\section{Assumptions related with the forward LIBOR rates}

1. Assume that for a predetermined collection of dates $0<T_{0}<T_{1}<\ldots<T_{n}$ with a fixed accrual period or tenor $\delta$, for any credit rate $c \in \mathcal{K} \backslash\{1\}$ and any $t \leq T_{i} \in\left[0, T^{\star}\right]$, we denote by $L\left(t, T_{i}, c\right)$ 
the forward rate for the interval from $T_{i}$ to $T_{i+1}$ as

$$
\begin{aligned}
1+\delta L\left(t, T_{i}, c\right): & =F_{B}\left(t, T_{i}, T_{i+1}, c\right) \\
: & =\frac{B\left(t, T_{i}, c\right)}{B\left(t, T_{i+1}, c\right)} \\
= & \exp \left\{\int_{T_{i}}^{T_{i+1}} f(t, s, c) d s\right\} \\
= & B\left(T_{i}, T_{i+1}, c\right)^{-1}
\end{aligned}
$$

where $B\left(t, T_{i}, c\right)$ represents the pre-default value of a corporate bond with zero recovery and credit rate $c \in \mathcal{K} \backslash\{1\}$, so that $B\left(t, T_{i}, c\right)=1_{\left\{\tau^{\star}>t\right\}} B\left(t, T_{i}, c\right) .^{2}$

2. Hence these simple forward rates should be contrasted with the instantaneous, continuously compounded defaultable forward rates (and short rates) in the framework of Heath, Jarrow and Morton (1992) which satisfy

$$
L\left(t, T_{i}, c\right)=\frac{1}{\delta}\left(\exp \left\{\int_{T_{i}}^{T_{i+1}} f(t, s, c) d s\right\}-1\right)
$$

Now we can define for any $0 \leq t \leq T_{i}$ and any $c \in \mathcal{K} \backslash\{1\}$ the forward LIBOR spread

$$
S_{c}\left(t, T_{i}\right):=L\left(t, T_{i}, c\right)-L\left(t, T_{i}\right)
$$

\section{Assumptions related with the Swap rates and Swaptions}

1. As usually, let us define a predetermined collection of dates $0<T_{1}<\ldots<T_{n}=T^{\star}$ with a fixed accrual period or tenor $\delta_{j}$, and any $t<T_{1} \in\left[0, T^{\star}\right]$ with $T^{\star}$ fixed. We first consider a fixedfor-floating forward start swap settled in arrears with notional principal $N$, usually equals to 1 , without loss of generality. We shall frequently refer to such a contract as the forward start payer swap. A long position in a forward start payer swap corresponds to the situation when an investor, between $T_{\alpha}$ and $T_{\beta}$ with $0<\alpha<\beta \leq n$, will make periodic payments determined by fixed interest rates $K_{\alpha, \beta}$, and will receive in exchange payments specified by some floating rate, usually $L\left(t, T_{j-1}\right)$. A short position in a forward start payer swap defines a closely related contract known as the forward start receiver swap.

2. Let us place ourselves within a framework of some arbitrage-free, or equivalently risk-neutral term structure model (under $\mathbb{P}^{\star}$ ). Then, the value at time $t$ of the forward start payer swap denoted by $F S_{t}$ or $F S_{t}\left(K_{\alpha, \beta}\right)$ equals

$$
\begin{aligned}
F S_{t}\left(K_{\alpha, \beta}\right) & =\mathbb{E}_{\mathbb{P}^{\star}}\left\{\sum_{j=\alpha}^{\beta} B\left(t, T_{j}\right)\left(L\left(t, T_{j-1}\right)-K_{\alpha, \beta}\right) \delta_{j}\right\} \\
& =\sum_{j=\alpha}^{\beta} \mathbb{E}_{\mathbb{P} \star}\left\{B\left(t, T_{j}\right)\left(B\left(T_{j-1}, T_{j}\right)^{-1}-\left(1+K_{\alpha, \beta} \delta_{j}\right)\right) \mid \mathcal{F}_{t}\right\}
\end{aligned}
$$

\footnotetext{
${ }^{2}$ Let us recall that the double sequence $\left(\tau_{k}, C_{\tau_{k}}\right)_{k \geq 1}$ is called a $\mathcal{C}$-adapted multivariate marked point process. Notice that the $\tau_{k}$ 's form a sequence of stopping times that define the moments of time that the credit rate $C$ changes. And more explicitly, the random variable (random stopping time) $\tau^{\star}$ will be defined as the moment of default so that $\tau^{\star}:=\inf \left\{t>\tau_{k-1} / C_{t}=1\right\} \wedge T^{\star}$. Further explanations can be find in Chapter 2 Section 2.1.2.
} 
where writing $c_{j}=1+k \delta_{j}$ and rearranging we obtain the following important result:

$$
\begin{aligned}
F S_{t}\left(K_{\alpha, \beta}\right) & =\sum_{j=\alpha}^{\beta}\left(B\left(t, T_{j-1}\right)-c_{j} B\left(t, T_{j}\right)\right) \\
& =B\left(t, T_{\alpha-1}\right)-\sum_{j=\alpha}^{\beta} c_{j} B\left(t, T_{j}\right)
\end{aligned}
$$

3. Alternatively, notice that the process $L\left(t, T_{j-1}\right)$ is a martingale under the forward measure $\mathbb{P}_{T_{j}}$. Then

$$
\begin{aligned}
F S_{t}\left(K_{\alpha, \beta}\right) & =\sum_{j=\alpha}^{\beta} B\left(t, T_{j}\right) \mathbb{E}_{\mathbb{P}_{T_{j}}}\left\{\left(L\left(t, T_{j-1}\right)-K_{\alpha, \beta}\right) \delta_{j}\right\} \\
& =\sum_{j=\alpha}^{\beta} B\left(t, T_{j}\right) \delta_{j}\left(L\left(t, T_{j-1}\right)-K_{\alpha, \beta}\right) \\
& =\sum_{j=\alpha}^{\beta}\left(B\left(t, T_{j-1}\right)-B\left(t, T_{j}\right)-K_{\alpha, \beta} \delta_{j} B\left(t, T_{j}\right)\right) \\
& =B\left(t, T_{\alpha-1}\right)-\sum_{j=\alpha}^{\beta} c_{j} B\left(t, T_{j}\right)
\end{aligned}
$$

where again $c_{j}=1+K \delta_{j}$ and we used the fact that

$$
L\left(t, T_{j-1}\right)=\frac{B\left(t, T_{j-1}\right)-B\left(t, T_{j}\right)}{\delta_{j} B\left(t, T_{j}\right)}
$$

Therefore, as we can observe in (3.6) and (3.7) a forward swap is essentially a contract to deliver a specific coupon-bearing bond and to receive at the same time a zero-coupon bond, and this relationship provides a simple method for the replication of a swap contract.

4. The forward swap rate $K_{\alpha, \beta}$ at time $t$ for the date $T_{\alpha}$ is that value of the fixed rate $K$ that makes the value of the n-period forward swap zero, i.e. that value of $K$ for which $F S_{t}(K)=0$

5. Let us define the payer (respectively, receiver) swaption with strike rate $K_{\alpha, \beta}$ as the financial derivative that gives to the owner the right to enter at time $T_{\alpha}$ the underlying forward payer (respectively, receiver) swap settled in arrears with maturity $T_{\beta}$, with $0<\alpha<\beta \leq n$. Because $F S_{T_{\alpha}}\left(K_{\alpha, \beta}\right)$ is the value at time $t$ of the forward payer swap with the fixed interest rate $K_{\alpha, \beta}$, it is clear that the price of the payer swaption at time $t$ equals

$$
P S\left(t, T_{\alpha}, T_{\beta}, \sigma_{\alpha, \beta}^{\star}, K_{\alpha, \beta}\right)=\mathbb{E}_{\mathbb{P}^{\star}}\left\{B\left(t, T_{\alpha}\right)\left(F S_{T_{\alpha}}\left(K_{\alpha, \beta}\right)\right)^{+} \mid \mathcal{F}_{t}\right\}
$$

where $t$ is the moment of valuation, $T_{\alpha}$ is the moment where the forward start swap begins and $T_{\beta}$ is the swap maturity, $\sigma_{\alpha, \beta}^{\star}$ is the implied volatility quoted in the swaption market for the strike $K_{\alpha, \beta}$. Therefore it is apparent that the option, in the payer swaption, is exercised at time $T_{\alpha}$ if and only if the value of the underlying forward swap with maturity $T_{\beta}$, is positive.

And for the receiver swaption we have

$$
R S\left(t, T_{\alpha}, T_{\beta}, \sigma_{\alpha, \beta}^{\star}, K_{\alpha, \beta}\right)=\mathbb{E}_{\mathbb{P}^{*}}\left\{B\left(t, T_{\alpha}\right)\left(-F S_{T_{\alpha}}\left(K_{\alpha, \beta}\right)\right)^{+} \mid \mathcal{F}_{t}\right\}
$$




\subsubsection{The LIBOR market model: a quick review}

Short rate models as well as term structure (Heath, Jarrow and Morton (1992) or HJM) models, like the Lévy term structure model discussed in the previous chapter, specify the dynamics of continuously compounded interest rates. From a mathematical point of view, these rates that apply for an infinitesimal time interval are very convenient for modelling purposes. However, interest rates quoted in real markets are usually effective (simply compounded). Sandmann, Sondermann, and Miltersen (1995), Miltersen, Sandmann, and Sondermann (1997), and Brace, Gatarek, and Musiela (1997) (or BGM) managed to incorporate a model for effective rates into an HJM-framework. An extended introduction to LIBOR models can be found in several textbooks and articles, e.g. in Musiela and Rutkowski (2004), Brigo and Mercurio (2001), or Rutkowski (2001).

The forward-LIBOR Model, which is often referred to as the LIBOR Market Model or BGM model, became a very popular approach among practitioners since it is consistent with the market practice of pricing caps and floors. Jamshidian (1999) generalized this model by considering semimartingales as driving processes, but pricing of caps and floors was not discussed in this setup. A model that lies in between Jamshidians's approach and the LIBOR market model as far as generality is concerned has recently been developed by Eberlein and Özkan (2005). Their Lévy LIBOR Market Model is more flexible than the usual LIBOR Market Model since it uses general Lévy processes as drivers instead of the special case of a Brownian motion. Moreover, explicit pricing formulae for caps and floors can be obtained.

The Brace, Gatarek, and Musiela (1997) or BGM model

The aim of this preliminary section is to give a brief introduction to the LIBOR Market Model or BGM model. A more comprehensive picture of the present knowledge can be obtained from Musiela and Rutkowski (2004)

To introduce formally the notion of a forward LIBOR, we assumed that we are given a family $B(t, T)$ for bond prices, and thus also the collection $F_{B}(t, T, U)$ of forward price processes. For a given horizon date $T^{\star}$ and a real positive number $\delta<T^{\star}$ representing the length of the accrual period, be fixed throughout.

By definition (see assumptions in section 3.1.2), the forward $\delta$-LIBOR rate $L(t, T)$ for the future date $T \leq T^{\star}-\delta$ prevailing at time $t$ is given by the conventional market formula

$$
\begin{aligned}
1+\delta L(t, T): & =F_{B}(t, T, T+\delta) \\
: & =\frac{B(t, T)}{B(t, T+\delta)} \\
= & \exp \left\{\int_{T}^{T+\delta} f(t, s) d s\right\}
\end{aligned}
$$

so that the forward LIBOR $L(t, T)$ represents in fact the add-on rate prevailing at time $t$ over the future time period $[T, T+\delta]$.

Following Brace, Gatarek and Musiela (1997), assume that we are given a family $F_{B}\left(t, T_{i}, T_{i+1}\right)$ of forward processes satisfying

$$
d F_{B}\left(t, T, T^{\star}\right)=F_{B}\left(t, T, T^{\star}\right) \gamma\left(t, T, T^{\star}\right) d W^{T^{\star}}
$$

on $\left(\Omega, \mathcal{F}_{t}, \mathbb{P}_{T^{\star}}\right)$, where $W^{T^{\star}}$ is a standard Brownian motion under $\mathbb{P}_{T^{\star}}$. 
Then, we can derive the dynamics of the associated family of forward LIBOR rates. For instance, one finds that under the forward measure $\mathbb{P}_{T+\delta}$ we have

$$
d L(t, T)=\delta^{-1} F_{B}(t, T, T+\delta) \gamma(t, T, T+\delta) d W^{T+\delta}
$$

where $W^{T+\delta}$ and $\mathbb{P}_{T+\delta}$ are defined by

$$
W_{t}^{T+\delta}=W^{T^{\star}}-\int_{0}^{t} \gamma(u, T, T+\delta) d u
$$

The process $W^{T+\delta}$ is a standard Brownian motion with respect the probability measure $\mathbb{P}_{T+\delta} \approx \mathbb{P}_{T^{\star}}$ defined on $\left(\Omega, \mathcal{F}_{t}\right)$ by means of the Radon-Nikodym density

$$
\frac{d \mathbb{P}_{T+\delta}}{d \mathbb{P}_{T^{\star}}}=\mathcal{E}_{T+\delta}\left(\int_{0}^{\cdot} \gamma(u, T, T+\delta) d W_{u}^{T^{\star}}\right)
$$

This means that $L(t, T)$ solves the equation

$$
d L(t, T)=\delta^{-1}(1+\delta L(t, T)) \gamma(u, T, T+\delta) d W_{t}^{T+\delta}
$$

Additionally let us assume that the forward LIBORs $L(t, T)$ are strictly positive. Then the last expression can be rewritten as follows

$$
d L(t, T)=L(t, T) \lambda(t, T) d W_{t}^{T+\delta}
$$

where for every $t \in[0, T]$ we have

$$
\lambda(t, T)=\frac{1+\delta L(t, T)}{\delta L(t, T)} \gamma(t, T, T+\delta)
$$

under the following assumptions:

- first, $\lambda(t, T)$ is a $\mathbb{R}^{d}$-valued bounded and $\mathcal{F}_{t}$-adapted process that represents the volatility of the forward LIBOR process $L(t, T)$

- and second, let us assume a strictly positive initial term structure $B(0, T)$ with $T \in\left[0, T^{\star}\right]$ and thus an initial term structure $L(0, T)$ of forward LIBORs

$$
L(0, T)=\frac{B(0, T)-B(0, T+\delta)}{\delta B(0, T+\delta)}, \text { for every } T \in\left[0, T^{\star}\right]
$$

Recall that the arbitrage-free dynamics of the instantaneous forward rate $f(t, T)$, according to the HJM model, are

$$
d f(t, T)=\sigma(t, T) \sigma^{\star}(t, T) d t+\sigma(t, T) d W_{t}
$$

and additionally we have

$$
1+\delta L(t, T)=\exp \left(\int_{T}^{T+\delta} f(t, u) d u\right)
$$

Applying Itô formula to both sides, and comparing the diffusion terms we find that

$$
\sigma^{\star}(t, T+\delta)-\sigma^{\star}(t, T)=\int_{T}^{T+\delta} \sigma(t, u) d u=\frac{\delta L(t, T)}{1+\delta L(t, T)} \lambda(t, T)
$$


Therefore we can conclude that the process $L(t, T)$ satisfies, under the spot martingale measure $\mathbb{P}$

$$
d L(t, T)=L(t, T) \sigma^{\star}(t, T) \lambda(t, T) d t+L(t, T) \lambda(t, T) d W_{t}
$$

or equivalently, under the forward measure $\mathbb{P}_{T+\delta}$

$$
d L(t, T)=L(t, T) \lambda(t, T) d W_{t}^{T+\delta}
$$

\section{The LIBOR market model with jumps or Lévy market model}

Eberlein and Özkan (2005) take two different approaches to model forward LIBOR rates in a discrete tenor setting. Both approaches have in common that they do not specify zero coupon bond prices directly (it is only assumed that the processes describing the evolution of bond prices are special semimartingales whose values as well as all left hand limits are strictly positive; moreover, the terminal value of each bond equals one). Instead, ratios of bond prices are specified. Eberlein and Özkan (2005) consider a fixed time horizon $T$ as well as a discrete tenor structure $0=T_{0}<T_{1}<\ldots<T_{n}=T$ and build up the model in one of the two following ways:

- The first approach, the Lévy LIBOR model, uses the (ordinary) exponential of a non-homogeneous Lévy process to model forward LIBOR rates directly, which are defined by

$$
L\left(t, T_{k}\right)=\frac{1}{\delta_{k}}\left(\frac{B\left(t, T_{k}\right)}{B\left(t, T_{k+1}\right)}-1\right) \text { for any } k \in\{1, \ldots, n-1\}
$$

where $\delta_{k}=T_{k+1}-T_{k}$.

- In the second approach, the Lévy forward price model, the mentioned authors define forward price process

$$
F_{B}\left(t, T_{k}, T_{k}+\delta_{k}\right):=\frac{B\left(t, T_{k}\right)}{B\left(t, T_{k+1}\right)} \text { for any } k \in\{1, \ldots, n-1\}
$$

are specified as starting value times the exponential of a non-homogeneous Lévy process. Notice that this immediately provides a model for forward LIBOR rates since $L\left(t, T_{k}\right)=\frac{1}{\delta_{k}}\left(F_{B}\left(t, T_{k}, T_{k+1}\right)-1\right)$. It is usually called the Lévy forward price model.

The Lévy LIBOR model The main aim of this subsection is to give a short overview over the Lévy LIBOR model. We are not going to present a construction of the model since this is done in Eberlein and Özkan (2005) in detail. Instead, we list some of the model properties that will be needed for option pricing in the subsequent sections as well as for the Lévy LIBOR model with credit risk which will be discussed in a later section.

The model is constructed by backward induction and driven by a Lévy process $L^{T^{\star}}$ on a complete stochastic basis $\left(\Omega, \mathcal{F}_{t}, \mathbb{P}_{T^{\star}}\right)$. As in the Lévy forward price model, $\mathbb{P}_{T^{\star}}$ should be regarded as the forward measure associated with the settlement day $T^{\star}$. Notice that $L^{T^{\star}}$ is required to be a martingale and can be written in its canonical decomposition as in Theorems $42-43$ in section 1.6, such that

$$
L_{t}^{T^{\star}}=\int_{0}^{t} \sigma_{s} d W_{s}^{T^{\star}}+\int_{0}^{t} \int_{\mathbb{R}^{d}} x\left(\mu-v^{T^{\star}}\right)(d s, d x)
$$

Here, $W_{s}^{T^{\star}}$ denotes a standard Brownian motion with respect to $\mathbb{P}_{T^{\star}}, v$ is the random measure associated with the jumps of $G_{t}^{T^{\star}}$ and $v^{T^{*}}(d t, d x)=F_{s}^{T^{\star}}(d x) d t$ is the compensator of $\mu$ with respect to $\mathbb{P}_{T^{\star}}$. The characteristics of $L_{s}^{T^{*}}$ are given by $\left(0, \sigma, F^{T^{\star}}\right)$. Note that without loss of generality we assume $L_{t}^{T^{\star}}$ to be driftless. 
Additionally, let us assume the following:

1. For any deterministic $T_{i}$ there is a deterministic function $\lambda\left(\cdot, T_{i}\right):\left[0, T_{i}\right] \rightarrow \mathbb{R}^{d}$ which represents the volatility of the forward rate process $L\left(t, T_{i}\right)$. In addition

$$
\sum_{i=1}^{n-1}\left|\lambda\left(s, T_{i}\right)\right| \leq M \text { for all } s \in\left[0, T^{\star}\right]
$$

where $M$ is the constant and $\lambda\left(s, T_{i}\right)=0$ for $s<T_{i}$.

2. The initial term structure $B\left(0, T_{i}\right)$ for any $i \in\{1, \ldots, n\}$ is strictly positive and strictly decreasing.

Therefore the dynamics of the forward LIBOR rates are specified as

$$
L\left(t, T_{k}\right)=L\left(0, T_{k}\right) \exp \left(\int_{0}^{t} b\left(s, T_{k}, T_{k+1}\right) d s+\int_{0}^{t} \lambda\left(t, T_{k}\right) d L_{s}^{T_{k}+1}\right)
$$

with initial condition

$$
L\left(0, T_{k}\right)=\frac{1}{\delta_{k}}\left(\frac{B\left(0, T_{k}\right)}{B\left(0, T_{k+1}\right)}-1\right)
$$

$L^{T_{k+1}}$ equals $L_{t}^{T^{\star}}$ plus some - in general non-deterministic - drift term which is chosen in such a way that $L^{T_{k+1}}$ is driftless under the forward measure associated with the settlement day $T_{k+1}$, henceforth denoted by $\mathbb{P}_{T_{k+1}}$. More precisely,

$$
L_{t}^{T_{k+1}}=\int_{0}^{t} \sigma_{s} d W_{s}^{T_{k+1}}+\int_{0}^{t} \int_{\mathbb{R}^{d}} x\left(\mu-v^{T_{k+1}}\right)(d s, d x)
$$

where $W_{s}^{T_{k+1}}$ is a standard Brownian motion with respect to $\mathbb{P}_{T_{k+1}}$ and $v^{T_{k+1}}$ is the $\mathbb{P}_{T_{k+1}}$-compensator of $\mu$. The drift term $b\left(s, T_{k}, T_{k+1}\right)$ is specified in such a way that $L\left(\cdot, T_{k}\right)$ becomes a $\mathbb{P}_{T_{k+1}}$-martingale, i. e.

$$
\begin{aligned}
b\left(s, T_{k}, T_{k+1}\right)= & -\frac{1}{2}\left\langle\lambda\left(s, T_{k}\right), \sigma_{s} \lambda\left(s, T_{k}\right)\right\rangle \\
& -\int_{\mathbb{R}^{d}}\left(e^{\left\langle\lambda\left(s, T_{k}\right), x\right\rangle}-1-\left\langle\lambda\left(s, T_{k}\right), x\right\rangle\right) F_{s}^{T_{k+1}}(d x)
\end{aligned}
$$

Notice that the connection between different forward measures is given by

$$
\frac{d \mathbb{P}_{T_{k+1}}}{d \mathbb{P}_{T^{\star}}}=\prod_{l=k+1}^{n-1} \frac{1+\delta_{l} L\left(T_{k+1}, T_{l}\right)}{1+\delta_{l} L\left(0, T_{l}\right)}=\frac{B\left(0, T^{\star}\right)}{B\left(0, T_{k+1}\right)} \prod_{l=k+1}^{n-1}\left(1+\delta_{l} L\left(T_{k+1}, T_{l}\right)\right)
$$

and notice that once restricted to the $\sigma$-field $\mathcal{F}_{t}$ this becomes

$$
\left.\frac{d \mathbb{P}_{T_{k+1}}}{d \mathbb{P}_{T^{\star}}}\right|_{\mathcal{F}_{t}}=\frac{B\left(0, T^{\star}\right)}{B\left(0, T_{k+1}\right)} \prod_{l=k+1}^{n-1}\left(1+\delta_{l} L\left(t, T_{l}\right)\right) \text { for every } t \in\left[0, T_{k+1}\right]
$$

The Brownian motions and compensators with respect to the different measures are connected via Girsanov theorem for semimartingales (see Theorem 48 in Chapter 1), such that

$$
W_{t}^{T_{k+1}}=W_{t}^{T^{\star}}-\int_{0}^{t} \sigma_{s}\left(\sum_{l=k+1}^{n-1} \alpha\left(s, T_{l}, T_{l+1}\right)\right) d s
$$


with

$$
\alpha\left(s, T_{l}, T_{l+1}\right):=\frac{\delta_{l} L\left(T_{k+1}, T_{l}\right)}{1+\delta_{l} L\left(s-, T_{l}\right)} \lambda\left(s, T_{l}\right)
$$

and

$$
\mu^{T_{k+1}}(d t, d x)=\left(\prod_{l=k+1}^{n-1} \beta\left(s, x, T_{l}, T_{l+1}\right)\right) \mu^{T^{\star}}(d t, d x)=: F_{s}^{T_{k+1}}(d x) d s
$$

where

$$
\beta\left(s, x, T_{l}, T_{l+1}\right):=\frac{\delta_{l} L\left(T_{k+1}, T_{l}\right)}{1+\delta_{l} L\left(s-, T_{l}\right)}\left(e^{\left\langle\lambda\left(s, T_{k}\right), x\right\rangle}-1\right)+1
$$

Notice that $L^{T_{k+1}}$ is not a Lévy process under any of the measures $\mathbb{P}_{T_{i}}$ (except for $k=n-1$ since $L^{T^{\star}}$ is by definition a Lévy process under $\mathbb{P}_{T^{\star}}$ ). However, the construction by backward induction guarantees that $\frac{B\left(\cdot, T_{j}\right)}{B\left(\cdot, T_{k}\right)}$ is a $\mathbb{P}_{T_{k}}$ martingale for all $j, k \in\{1, \ldots, n\}$.

The Lévy forward price model The Lévy forward price model is constructed by backward induction. It is driven by a Lévy process $L^{T^{\star}}$ (see section 1.1.3) on a complete stochastic basis $\left(\Omega, \mathcal{F}_{t}, \mathbb{P}_{T^{\star}}\right.$ ). The measure $\mathbb{P}_{T^{\star}}$ plays the role of the forward measure associated with the settlement day $T^{\star}$. Two of the characteristics $\left(b^{T^{\star}}, \sigma, F^{T^{\star}}\right)$ of $L^{T^{\star}}$ can be chosen freely, namely $\sigma$ and $F^{T^{\star}}$, whereas the drift characteristic $b^{T^{\star}}$ will be derived later. Since we proceed by backward induction, let us denote $T^{\star}:=T_{n}$ and $\delta:=T_{n-i+1}-T_{n-i}$ for any $i \in\{0, \ldots, n\}$. As in the previous model we have to add the following assumptions:

1. For any deterministic $T_{i}$ there is a deterministic function $\lambda\left(\cdot, T_{i}\right):\left[0, T_{i}\right] \rightarrow \mathbb{R}^{d}$ which represents the volatility of the forward rate process $L\left(t, T_{i}\right)$. In addition

$$
\sum_{i=1}^{n-1}\left|\lambda\left(s, T_{i}\right)\right| \leq M \text { for all } s \in\left[0, T^{\star}\right]
$$

where $M$ is the constant and $\lambda\left(s, T_{i}\right)=0$ for $s<T_{i}$.

2. The initial term structure $B\left(0, T_{i}\right)$ for any $i \in\{1, \ldots, n\}$ is strictly positive and strictly decreasing.

We begin by constructing the forward price with the longest maturity and postulate that

$$
F\left(t, T_{n-1}, T^{\star}\right)=F\left(0, T_{n-1}, T^{\star}\right) \exp \left(\int_{0}^{t} \lambda\left(s, T_{n-1}\right) d L_{s}^{T^{\star}}\right)
$$

subject to the initial condition

$$
F\left(0, T_{n-1}, T^{\star}\right)=\frac{B\left(0, T_{n-1}\right)}{B\left(0, T^{\star}\right)}
$$

Notice that an equivalent way to write it is in terms of the forward LIBOR rate

$$
1+\delta L\left(t, T_{i}\right):=1+\delta L\left(0, T_{i}\right) \exp \left(\int_{0}^{t} \lambda\left(s, T_{n-1}\right) d L_{s}^{T^{\star}}\right)
$$

Our goal is to specify the drift characteristic $b^{T^{*}}$ in such a way that the forward price process $F\left(\cdot, T_{n-1}, T^{\star}\right)$ (or equivalently the forward LIBOR rate $L\left(\cdot, T_{n-1}\right)$ ) is a martingale with respect to $\mathbb{P}_{T^{\star}}$ 
. For this purpose, we choose $b^{T^{\star}}$ such that

$$
\begin{aligned}
\int_{0}^{t}\left\langle\lambda\left(s, T_{n-1}\right), b_{s}^{T^{\star}}\right\rangle d s= & -\frac{1}{2} \int_{0}^{t}\left\langle\lambda\left(s, T_{n-1}\right), \sigma_{s} \lambda\left(s, T_{n-1}\right)\right\rangle d s \\
& -\int_{0}^{t} \int_{R^{d}}\left(e^{\left\langle\lambda\left(s, T_{n-1}\right), x\right\rangle}-1-\left\langle\lambda\left(s, T_{n-1}\right), x\right\rangle\right) v^{T^{\star}}(d s, d x)
\end{aligned}
$$

where $v^{T^{\star}}(d s, d x):=F_{s}^{T^{\star}}(d x) d s$ is the compensator of the random measure $\mu$ that is associated with the jumps of $L^{T^{\star}}$. Lemma 2.6 in Kallsen and Shiryaev (2002) yields that the forward price $F\left(\cdot, T_{n-1}, T^{\star}\right)$ can then be expressed as the stochastic exponential of a local martingale, namely

$$
F\left(t, T_{n-1}, T^{\star}\right)=F\left(0, T_{n-1}, T^{\star}\right) \mathcal{E}_{t}\left(H\left(\cdot, T_{n-1}\right)\right)
$$

with

$$
\begin{aligned}
H\left(t, T_{n-1}\right)= & \int_{0}^{t} \sigma_{s} \lambda\left(s, T_{n-1}\right) d W_{s}^{T^{\star}} \\
& +\int_{0}^{t} \int_{R^{d}}\left(e^{\left\langle\lambda\left(s, T_{n-1}\right), x\right\rangle}-1\right)\left(v-\mu^{T^{\star}}\right)(d s, d x)
\end{aligned}
$$

Note that $H\left(t, T_{n-1}\right)$ is also a Lévy process. The stochastic exponential of a process that is a local martingale as well as a Lévy process is not only a local martingale, but in fact a martingale (see e.g. Eberlein, Jacod, and Raible (2005) for a proof). Hence, $F\left(t, T_{n-1}, T^{\star}\right)$ and thus also $L\left(t, T_{n-1}\right)$ are martingales.

We define the forward martingale measure associated with the date $T_{n-1}$ by setting

$$
\frac{d \mathbb{P}_{T_{n-1}}}{d \mathbb{P}_{T^{\star}}}:=\frac{F\left(T_{n-1}, T_{n-1}, T^{\star}\right)}{F\left(0, T_{n-1}, T^{\star}\right)}=\mathcal{E}_{T_{n-1}}\left(H\left(\cdot, T_{n-1}\right)\right)
$$

From equation (3.11) we can immediately identify the two predictable processes $\beta$ and $Y$ in the Girsanov's theorem for semimartingales (see Theorem 48 in Chapter 1) that describe the change of measure, namely

$$
\beta(s)=\lambda\left(s, T_{n-1}\right) \quad \text { and } \quad Y(s, x)=\exp \left\langle\lambda\left(s, T_{n-1}\right), x\right\rangle
$$

Notice that $W_{t}^{T_{n-1}}:=W_{t}^{T^{\star}}-\int_{0}^{t} \sigma_{s} \lambda\left(s, T_{n-1}\right) d s$ is a standard Brownian motion with respect to $\mathbb{P}_{T_{n-1}}$ and $v^{T_{n-1}}(d s, d x):=\exp \left\langle\lambda\left(s, T_{n-1}\right), x\right\rangle v^{T^{\star}}(d s, d x)$ is the $\mathbb{P}_{T_{n-1}}$ - compensator of $\mu$. Therefore, we have the following $\mathbb{P}_{T_{n-1}}$-canonical representation of $L_{t}^{T^{\star}}$ :

$$
L_{t}^{T^{\star}}=\int_{0}^{t} b_{s} d s+\int_{0}^{t} \sigma_{s} d W_{s}^{T_{n-1}}+\int_{0}^{t} \int_{R^{d}} x\left(\mu-v^{T_{n-1}}\right)(d s, d x)
$$

with a deterministic drift coefficient $b$ which can be calculated using the Girsanov's Theorem.

Now we are ready to construct the forward price $F\left(\cdot, T_{n-2}, T_{n-1}\right)$ by postulating that

$$
F\left(t, T_{n-2}, T_{n-1}\right)=F\left(0, T_{n-2}, T_{n-1}\right) \exp \left(\int_{0}^{t} \lambda\left(s, T_{n-2}\right) d L_{s}^{T_{n-1}}\right)
$$

where

$$
L_{t}^{T_{n-1}}=\int_{0}^{t} b_{s}^{T_{n-1}} d s+\int_{0}^{t} \sigma_{s} d W_{s}^{T_{n-1}}+\int_{0}^{t} \int_{\mathbb{R}^{d}} x\left(\mu-v^{T_{n-1}}\right)(d s, d x)
$$


In order to ensure that $F\left(t, T_{n-2}, T_{n-1}\right)$ is a $\mathbb{P}_{T_{n-1}}$-martingale, we choose the drift characteristic $b_{s}^{T_{n-1}}$ appropriately, namely such that

$$
\begin{aligned}
\int_{0}^{t}\left\langle\lambda\left(s, T_{n-2}\right), b_{s}^{T_{n-1}}\right\rangle d s= & -\frac{1}{2} \int_{0}^{t}\left\langle\lambda\left(s, T_{n-2}\right), \sigma_{s} \lambda\left(s, T_{n-2}\right)\right\rangle d s \\
& -\int_{0}^{t} \int_{\mathbb{R}^{d}}\left(e^{\left\langle\lambda\left(s, T_{n-2}\right), x\right\rangle}-1-\left\langle\lambda\left(s, T_{n-2}\right), x\right\rangle\right) v^{T_{n-1}}(d s, d x)
\end{aligned}
$$

Note that $L_{t}^{T_{n-1}}$ differs from $L_{t}^{T^{\star}}$ only by a deterministic drift term. In particular, both processes are Lévy processes under $\mathbb{P}_{T^{\star}}$ and $\mathbb{P}_{T_{n-1}}$. Again, we can express the forward price process $F\left(t, T_{n-2}, T_{n-1}\right)$ as the stochastic exponential of a Lévy process and local martingale $H\left(t, T_{n-2}\right)$ and use the martingale $\left(\frac{F\left(t, T_{n-2}, T_{n-1}\right)}{F\left(0, T_{n-2}, T_{n-1}\right)}\right)_{0 \leq t \leq T_{n-2}}$ to define the forward martingale measure associated with the date $T_{n-2}$ by setting

$$
\frac{d \mathbb{P}_{T_{n-1}}}{d \mathbb{P}_{T^{\star}}}:=\frac{F\left(T_{n-2}, T_{n-2}, T_{n-1}\right)}{F\left(0, T_{n-1}, T_{n-1}\right)}
$$

And proceeding as before, forward prices $F\left(\cdot, T_{i}, T_{i+1}\right)$ for $i=n-1, \ldots, 3$ and forward measures $\mathbb{P}_{T_{i}}$ for $i=n-2, \ldots, 2$ are defined inductively. We obtain a model where the forward price $F\left(\cdot, T_{i}, T_{i+1}\right)$ is given by

$$
F\left(t, T_{i}, T_{i+1}\right)=F\left(0, T_{i}, T_{i+1}\right) \exp \left(\int_{0}^{t} \lambda\left(s, T_{i}\right) d L_{s}^{T_{i-1}}\right)
$$

with

$$
L_{t}^{T_{i-1}}=\int_{0}^{t} b_{s}^{T_{i-1}} d s+\int_{0}^{t} \sigma_{s} d W_{s}^{T_{i-1}}+\int_{0}^{t} \int_{\mathbb{R}^{d}} x\left(\mu-v^{T_{i-1}}\right)(d s, d x)
$$

where $W_{t}^{T_{i-1}}$ is a $\mathbb{P}_{T_{i-1}}$-standard Brownian motion and $v^{T_{i-1}}$ is the $\mathbb{P}_{T_{i-1}}$-compensator of $\mu$, given by

$$
v^{T_{i-1}}(d s, d x)=\exp \left(\sum_{j=1}^{i-1}\left\langle\lambda\left(s, T_{j}^{\star}\right), x\right\rangle\right) F_{t}^{T^{\star}}(d x) d s
$$

and the characteristic $b^{T_{i-1}}$ satisfies

$$
\begin{aligned}
\int_{0}^{t}\left\langle\lambda\left(s, T_{i-2}\right), b_{s}^{T_{i-1}}\right\rangle d s= & -\frac{1}{2} \int_{0}^{t}\left\langle\lambda\left(s, T_{i-2}\right), \sigma_{s} \lambda\left(s, T_{i-2}\right)\right\rangle d s \\
& -\int_{0}^{t} \int_{\mathbb{R}^{d}}\left(e^{\left\langle\lambda\left(s, T_{i-2}\right), x\right\rangle}-1-\left\langle\lambda\left(s, T_{i-2}\right), x\right\rangle\right) v^{T_{i-1}}(d s, d x)
\end{aligned}
$$

Notice that the driving processes $L_{t}^{T_{i}}$ differ only by deterministic drift terms. Hence, all of them are Lévy processes with respect to each forward measure. 


\subsection{The LIBOR Market Model driven by a LIBOR additive process}

Under the conditions and assumptions given in sections 3.1.2 and 3.1.3, in the present section, we characterize the functional forms of the LIBOR Market Model (LMM) driven by a LIBOR additive process, and more specifically, when we consider the existence of credit risk. Basically our aim in this section is to recover the functional form of LMM under different martingale measures with credit risk.

LMMs have been developed in Brace, Gatarek, and Musiela (1997), Miltersen, Sandmann, and Sondermann (1997), and Jamshidian (1997) to construct an arbitrage-free term structure model which is consistent with the market practice of pricing caps and floors by Black's formula. Black's formula for caps is motivated by the option formula of Black and Scholes (1973) and implies that the LIBOR rates follow a geometric Brownian motion. We propose a more general model where the driving process of the LIBOR rates is a general Lévy process instead of a Brownian motion. An introduction to LIBOR models can be found in several textbooks and articles, e.g. in Musiela and Rutkowski (1998), Brigo and Mercurio (2001), or Rutkowski (2001).

This chapter is linked to the recent developments given by Glasserman and Kou (2001), Jamshidian (1999) and to Eberlein and Özkan (2005). In Glasserman and Kou (2001) the term structure of LIBOR rates is driven by a jump diffusion process. In this case the purely discontinuous part is of bounded variation. In Jamshidian (1999) the LIBOR rate process is driven by a general semimartingale, but the pricing is not considered. Basically, it is in Eberlein and Özkan (2005) where the LIBOR market model is driven by Lévy processes, and using Björk, Di Masi, Kabanov, and Runggaldier (1997), they derive the functional forms for the LMM under different martingale measures. This chapter can be seen in parts as a special case of the Eberlein and Özkan approach, but we specify the driving process as a LIBOR additive process.

This section is organized as follows:

- Subsection 1 is devoted to introduce the concept of change of measure in the LIBOR market model driven by a LIBOR additive process. Following closely Björk, Di Masi, Kabanov, and Runggaldier (1997) we introduce the basic tools to change the measure in this new framework.

- Basically the next subsections are examples of the first, in the sense that we obtain the functional form of LMM driven by LIBOR additive process, under risk neutral measure.

- And it is in subsection 3 where we introduce the concept of forward neutral measure and we obtain specific functional forms for LMM driven by LIBOR additive process, under forward neutral measure.

\subsubsection{General framework: an introduction to the extended LMM}

This first section introduces the basic tools to proceed with the change of measure under the framework of forward LMMs. Basically it is a direct application of the Girsanov's theorem already mentioned and described in Chapter 1 (Theorem 48)

Assume that we are given a family of $B\left(t, T_{i}, c\right)$ defaultable bond prices and thus also the collection $F_{B}\left(t, T_{i}, U, c\right)$ of forward price processes. According to Section 3.1.2, we have seen that the forward price $F_{B}\left(t, T_{i}, T^{\star}, c\right)$ for the future date $T_{i} \leq T^{\star}$ prevailing at time $t$ and for any credit rate $c \in \mathcal{K} \backslash\{1\}$, is 
given by the well-known expression

$$
\begin{aligned}
F_{B}\left(t, T_{i}, T^{\star}, c\right) & =\frac{B\left(t, T_{i}, c\right)}{B\left(t, T^{\star}, c\right)} \\
& =\exp \left\{\sum_{i \leq j \leq n} \int_{T_{i}}^{T_{i+1}} f(t, s, c) d s\right\}
\end{aligned}
$$

Assume that, under $\mathbb{P}_{\star}$, we are given a family $F_{B}\left(t, T_{i}, T^{\star}, c\right)$ of forward processes satisfying

$$
d F_{B}\left(t, T_{i}, T^{\star}, c\right)=F_{B}\left(t-, T_{i}, T^{\star}, c\right) \cdot d G_{t}^{c, T^{\star}}
$$

with initial condition

$$
F_{B}\left(0, T_{i}, T^{\star}, c\right)=\frac{B\left(0, T_{i}, c\right)}{B\left(0, T^{\star}, c\right)}
$$

Notice that assuming that $\mathbb{P}_{i} \approx \mathbb{P}_{\star}$, and by Jacod and Shiryaev (2003) (Theorems III.3.24, III.5.19, $I I I .5 .35)$, we can redefine $F_{B}\left(t, T_{i}, T^{\star}, c\right)$ under any equivalent forward measure $\mathbb{P}_{i}$ as

$$
F_{B}\left(t, T_{i}, T^{\star}, c\right)=F_{B}\left(0, T_{i}, T^{\star}, c\right) \cdot \mathcal{E}\left(G_{t}^{c, T_{i}, T^{\star}}\right)
$$

where $\mathcal{E}\left(G_{t}^{c, T_{i}, T^{\star}}\right)$ is the the Doléans-Dade exponential. More specifically, there exists a deterministic sequence of processes $\left(\beta_{j}(t)\right)$ and a measurable deterministic sequence $\left(Y_{j}(t)\right)$

$$
\left\{\begin{array}{l}
\beta=\left(\beta_{j}(t)\right), \text { a sequence of predictable processes } \\
Y=\left(Y_{j}(t)\right), \text { a sequence of } \mathcal{P} \text {-measurable nonnegative functions }
\end{array}\right.
$$

called Girsanov's quantities such that

$$
\begin{aligned}
F_{B}\left(\cdot, T_{i}, T^{\star}, c\right)= & F_{B}\left(0, T_{i}, T^{\star}, c\right) \exp \left(\sum_{j \leq \eta(t)} \int_{T_{j-1}}^{T_{j} \wedge t} \beta_{j}\left(s, T_{i}, T^{\star}, c\right) d W_{s}^{T^{\star}}\right. \\
& -\frac{1}{2} \sum_{j \leq \eta(t)} \int_{T_{j-1}}^{T_{j} \wedge t} \beta_{j}^{2}\left(s, T_{i}, T^{\star}, c\right) d s \\
& +\sum_{j \leq \eta(t)} \int_{T_{j-1}}^{T_{j} \wedge t} \int_{\mathbb{R}^{d}}\left(Y_{j}\left(s, T_{i}, T^{\star}, c\right)-1\right)\left(\mu_{j}^{c}-v_{j}^{c, T^{\star}}\right)(d t, d x) \\
& \left.+\sum_{j \leq \eta(t)} \int_{T_{j-1}}^{T_{j} \wedge t} \int_{\mathbb{R}^{d}}\left(Y_{j}\left(s, T_{i}, T^{\star}, c\right)-1-\ln Y_{j}\left(s, T_{i}, T^{\star}, c\right)\right) \mu_{j}^{c, T^{\star}}(d t, d x)\right)
\end{aligned}
$$

Furthermore, recall that the notion of forward $\delta$-LIBOR rate $L\left(t, T_{i}, c\right)$ for the future date $T_{i} \leq T_{i}-\delta$ prevailing at time time $t$ and for any credit rate $c \in \mathcal{K} \backslash\{1\}$, is given by the well-known formula

$$
1+\delta L\left(t, T_{i}, c\right):=F_{B}\left(t, T_{i}, T_{i+1}, c\right)
$$

and notice that the forward process can be written as

$$
F_{B}\left(t, T_{i}, T_{i+1}, c\right)=\frac{F_{B}\left(t, T_{i}, T^{\star}, c\right)}{F_{B}\left(t, T_{i+1}, T^{\star}, c\right)}
$$


hence

$$
\begin{aligned}
F_{B}\left(t, T_{i}, T_{i+1}, c\right)= & F_{B}\left(0, T_{i}, T_{i+1}, c\right) \exp \left(\sum_{j \leq \eta(t)} \int_{T_{j-1}}^{T_{j} \wedge t} \beta_{j}\left(s, T_{i}, T_{i+1}, c\right) d W_{s}^{T^{\star}}\right. \\
& -\frac{1}{2} \sum_{j \leq \eta(t)} \int_{T_{j-1}}^{T_{j} \wedge t}\left(\beta_{j}^{2}\left(s, T_{i}, T^{\star}, c\right)-\beta_{j}^{2}\left(s, T_{i+1}, T^{\star}, c\right)\right) d s \\
& +\sum_{j \leq \eta(t)} \int_{T_{j-1}}^{T_{j} \wedge t} \int_{\mathbb{R}^{d}}\left(Y_{j}\left(s, T_{i}, T_{i+1}, c\right)-1\right)\left(\mu_{j}^{c}-v_{j}^{c, T^{\star}}\right)(d t, d x) \\
& \left.+\sum_{j \leq \eta(t)} \int_{T_{j-1}}^{T_{j} \wedge t} \int_{\mathbb{R}^{d}}\left(\ln \left(Y_{j}^{\prime}\left(s, T_{i}, T_{i+1}, c\right)\right)-Y_{j}\left(s, T_{i}, T_{i+1}, c\right)+1\right) \mu_{j}^{c, T^{\star}}(d t, d x)\right)
\end{aligned}
$$

where we have to substitute

$$
\begin{aligned}
\beta_{j}\left(s, T_{i}, T_{i+1}, c\right) & =\beta_{j}\left(s, T_{i}, T^{\star}, c\right)-\beta_{j}\left(s, T_{i+1}, T^{\star}, c\right) \\
Y_{j}\left(s, T_{i}, T_{i+1}, c\right) & =Y_{j}\left(s, T_{i}, T^{\star}, c\right)-Y_{j}\left(s, T_{i+1}, T^{\star}, c\right) \\
Y_{j}^{\prime}\left(s, T_{i}, T_{i+1}, c\right) & =\frac{1+Y_{j}\left(s, T_{i}, T^{\star}, c\right)}{1+Y_{j}\left(s, T_{i+1}, T^{\star}, c\right)}
\end{aligned}
$$

The $\mathbb{P}_{T^{\star}}$ dynamics of $F_{B}\left(\cdot, T_{i}, T_{i+1}, c\right)$ can again be derived by Itô formula

$$
\begin{aligned}
d F_{B}\left(t, T_{i}, T_{i+1}, c\right)= & F_{B}\left(t-, T_{i}, T_{i+1}, c\right)\left(\beta_{j}\left(s, T_{i}, T_{i+1}, c\right)\left(d W_{s}^{T^{\star}}-\beta_{j}\left(s, T_{i}, T^{\star}, c\right) d t\right)\right) \\
& +\int_{\mathbb{R}^{d}}\left(Y_{j}\left(s, T_{i}, T_{i+1}, c\right)-1\right)\left(\mu_{j}^{c}-v_{j}^{c, T^{\star}}\right)(d t, d x) \\
& \left.+\int_{\mathbb{R}^{d}}\left(Y_{j}^{\prime}\left(s, T_{i}, T_{i+1}, c\right)-Y_{j}\left(s, T_{i}, T_{i+1}, c\right)-1\right) \mu_{j}^{c, T^{\star}}(d t, d x)\right)
\end{aligned}
$$

Notice that using Girsanov's theorem (see Chapter 1, Theorem 48), we can define a measure $\mathbb{P}_{i} \approx \mathbb{P}_{\star}$ with the property

$$
W_{t}^{T_{i}}:=W_{t}^{T^{\star}}-\sum_{j \leq \eta(t)} \int_{T_{j-1}}^{T_{j} \wedge t} \beta_{j}\left(s, T_{i}, T_{i+1}, c\right) d s
$$

and

$$
v_{j}^{c, T_{i}}(d t, d x):=\left(Y_{j}\left(s, T_{i+1}, T^{\star}, c\right)-1\right) v_{j}^{c, T^{\star}}(d t, d x)
$$

hence under $\mathbb{P}_{i}$

$$
\begin{aligned}
d F_{B}\left(t, T_{i}, T_{i+1}, c\right)= & F_{B}\left(t-, T_{i}, T_{i+1}, c\right)\left(\beta_{j}\left(s, T_{i}, T_{i+1}, c\right) d W_{s}^{T_{i}}\right. \\
& \left.+\int_{\mathbb{R}^{d}}\left(Y_{j}^{\prime}\left(s, T_{i}, T_{i+1}, c\right)-1\right)\left(\mu_{j}^{c}-v_{j}^{c, T_{i}}\right)(d t, d x)\right)
\end{aligned}
$$

is a martingale. 
Let us study now the dynamics of the forward LIBOR rates under $\mathbb{P}_{i}$

$$
\begin{aligned}
d L\left(t, T_{i}, c\right)= & \frac{1}{\delta} d F_{B}\left(t, T_{i}, T_{i+1}, c\right) \\
= & \frac{1}{\delta} F_{B}\left(t-, T_{i}, T_{i+1}, c\right)\left(\beta_{j}\left(s, T_{i}, T_{i+1}, c\right) d W_{s}^{T_{i}}\right. \\
& \left.+\int_{\mathbb{R}^{d}}\left(Y_{j}^{\prime}\left(s, T_{i}, T_{i+1}, c\right)-1\right)\left(\mu_{j}^{c}-v_{j}^{c, T_{i}}\right)(d t, d x)\right) \\
= & \frac{1}{\delta}\left(1+\delta L\left(t-, T_{i}, c\right)\right)\left(\beta_{j}\left(s, T_{i}, T_{i+1}, c\right) d W_{s}^{T_{i}}\right. \\
& \left.+\int_{\mathbb{R}^{d}}\left(Y_{j}^{\prime}\left(s, T_{i}, T_{i+1}, c\right)-1\right)\left(\mu_{j}^{c}-v_{j}^{c, T_{i}}\right)(d t, d x)\right)
\end{aligned}
$$

hence under $\mathbb{P}_{i}$

$$
d L\left(t, T_{i}, c\right)=L\left(t-, T_{i}, c\right)\left(\theta_{j}^{1}\left(s, T_{i}, c\right) d W_{s}^{T_{i}}+\int_{\mathbb{R}^{d}} \theta_{j}^{2}\left(s, T_{i}, c\right)\left(\mu_{j}^{c}-v_{j}^{c, T_{i}}\right)(d t, d x)\right)
$$

where

$$
\begin{aligned}
\theta_{j}^{1}\left(s, T_{i}, c\right) & =\frac{1+\delta L\left(t-, T_{i}, c\right)}{\delta L\left(t-, T_{i}, c\right)} \beta_{j}\left(s, T_{i}, T_{i+1}, c\right) \\
\theta_{j}^{2}\left(s, T_{i}, c\right) & =\frac{1+\delta L\left(t-, T_{i}, c\right)}{\delta L\left(t-, T_{i}, c\right)}\left(Y_{j}^{\prime}\left(s, T_{i}, T_{i+1}, c\right)-1\right)
\end{aligned}
$$

\subsubsection{No-arbitrage conditions under the risk-neutral measure}

Our purpose, in the present section, is to give the appropriate drift-restrictions in order to find the LIBOR rate dynamic under the martingale (or equivalently, a risk-neutral) measure, for a given credit level of rating $c \in \mathcal{K} \backslash\{1\}$.

Recall that the tenor $\delta>0$, with $\delta \leq T^{\star}-T_{i}$ for any $i=0,1, \ldots, n$ then the $\delta$-forward LIBOR rates defined (see section 3.1) by

$$
\begin{aligned}
L\left(t, T_{i}, c\right) & =\frac{1}{\delta}\left(F_{B}\left(t, T_{i}, T_{i+1}, c\right)-1\right) \\
& =\frac{1}{\delta}\left(\frac{B\left(t, T_{i}, c\right)}{B\left(t, T_{i+1}, c\right)}-1\right)
\end{aligned}
$$

The $\delta$ - forward LIBOR rate coincides with the forward swap rate of a single period swap settled in arrears. The next theorem is an adaptation of Glasserman and Kou (2003), and Eberlein and Özkan (2005) and it states the dynamics of $L\left(t, T_{i}, c\right)$ under the risk-neutral measure.

Theorem 101 If $f(t, T, c)$ satisfies the dynamics that appear in (2.36) then the dynamics of $L\left(t, T_{i}, c\right)$ under $\mathbb{Q}$ (risk-neutral probability) is given by

$$
\begin{aligned}
\frac{\delta}{1+\delta L\left(t, T_{i}, c\right)} d L\left(t, T_{i}, c\right)= & \sigma_{i}^{*} \sum_{\eta(t) \leq j \leq i} \sigma_{j}^{*} d t+\sigma_{i}^{*} d W_{u} \\
& +\int_{\mathbb{R}^{r}}\left(e^{\delta_{i}^{*}}-1+e^{\sum_{\eta(t) \leq j \leq i} \delta_{j}^{*}}\left(e^{\delta_{i+1}^{*}}-1\right)\right) v_{i}^{c}(d t, d x) \\
& +\int_{\mathbb{R}^{r}}\left(e^{\delta_{i}^{*}}-1\right)\left(\mu_{i}^{c}-v_{i}^{c}\right)(d t, d x)
\end{aligned}
$$


with

$$
\begin{aligned}
\sigma_{i}^{*} & =\int_{T_{i}}^{T_{i}+\delta} \sigma_{i}(t, s, c) d s \\
\delta_{i}^{*} & =\int_{T_{i}}^{T_{i}+\delta} \delta_{i}(t, x, s) d s
\end{aligned}
$$

Proof. Following similar ideas as Eberlein and Özkan (2005), we get

$$
\begin{aligned}
F_{B}\left(t, T_{i}, T_{i}+\delta, c\right)= & 1+\delta L\left(t, T_{i}, c\right) \\
= & \frac{B\left(t, T_{i}, c\right)}{B\left(t, T_{i+1}, c\right)} \\
= & \frac{B\left(0, T_{i}, c\right)}{B\left(0, T_{i}+\delta, c\right)} \exp \left[\sum_{j \leq \eta(t)} \int_{T_{j}}^{t \wedge T_{j+1}}\left(a_{j}\left(u, T_{i}, c\right)-a_{j}\left(u, T_{i+1}, c\right)\right) d u\right. \\
& +\sum_{j \leq \eta(t)} \int_{T_{j}}^{t \wedge T_{j+1}}\left(b_{j}\left(u, T_{i}, c\right)-b_{j}\left(u, T_{i+1}, c\right)\right) d W_{u} \\
& \left.+\sum_{j \leq \eta(t)} \int_{T_{j}}^{t \wedge T_{j+1}} \int_{\mathbb{R}^{d}}\left(h\left(u, x, T_{i}\right)-h\left(u, x, T_{i+1}\right)\right) 1_{\{|x| \geq 1\}} v_{j}^{c}(d u, d x)\right]
\end{aligned}
$$

We know that the dynamic of the LIBOR rate is related to the dynamics of the forward process in the following way

$$
\frac{\delta d L\left(t, T_{i}, c\right)}{1+\delta L\left(t, T_{i}, c\right)}=\frac{d F_{B}\left(t, T_{i}, T_{i+1}, c\right)}{F_{B}\left(t-, T_{i}, T_{i+1}, c\right)}
$$

Since $F_{B}\left(t, T_{i}, T_{i+1}, c\right)$ is a positive semimartingale, it can be expressed as the stochastic exponential of the stochastic logarithm

$$
\log \left(F_{B}\left(t, T_{i}, T_{i+1}, c\right)\right)=\int d F_{B}\left(t, T_{i}, T_{i+1}, c\right) / F_{B}\left(t-, T_{i}, T_{i+1}, c\right)
$$

and this process can be represented as follows (based on Kallsen and Shiryaev (2002) Lemma 2.4 and Jacod and Shiryaev, Theorem II.8.3):

$$
\begin{aligned}
\log \left(F_{B}\left(t, T_{i}, T_{i+1}, c\right)\right)= & \log \left(\frac{F_{B}\left(t, T_{i}, T_{i+1}, c\right)}{F_{B}\left(0, T_{i}, T_{i+1}, c\right)}\right) \\
& +\sum_{j \leq \eta(t)} \int_{T_{j}}^{t \wedge T_{j+1}} \frac{1}{2 F_{B}^{2}\left(s-, T_{i}, T_{i+1}, c\right)} d\left\langle F_{B}^{c}\left(\cdot, T_{i}, T_{i+1}, c\right), F_{B}^{c}\left(\cdot, T_{i}, T_{i+1}, c\right)\right\rangle \\
& -\sum_{j \leq \eta(t)} \int_{T_{j}}^{t \wedge T_{j+1}} \int_{\mathbb{R}^{r}}\left(\log \left(\left|1+\frac{x}{F_{B}\left(s-, T_{i}, T_{i+1}, c\right)}\right|\right)\right. \\
& \left.-\frac{x}{F_{B}\left(s-, T_{i}, T_{i+1}, c\right)}\right) \mu_{j}^{F_{B}, c}(d s, d x)
\end{aligned}
$$

Following Eberlein and Özkan (2005) and introducing the martingale assumption (EMM) we have 
that

$$
\begin{aligned}
\frac{d F_{B}\left(t, T_{i}, T_{i+1}\right)}{F_{B}\left(t-, T_{i}, T_{i+1}\right)}= & \left(b_{j}\left(t, T_{i}, c\right)-b_{j}\left(t, T_{i+1}, c\right)\right) b_{j}\left(t, T_{i+1}, c\right) d t \\
& +\left(b_{j}\left(t, T_{i}, c\right)-b_{j}\left(t, T_{i+1}, c\right)\right) d W_{t} \\
& +\int_{\mathbb{R}^{r}}\left(e^{h\left(t, x, T_{i}\right)-h\left(t, x, T_{i}+\delta\right)}-1+e^{h\left(t, x, T_{i}+\delta\right)}-e^{h\left(t, x, T_{i}\right)}\right) v_{i}^{c}(d t, d x) \\
& +\int_{\mathbb{R}^{r}}\left(e^{h\left(t, x, T_{i}\right)-h\left(t, x, T_{i}+\delta\right)}-1\right)\left(\mu_{i}^{c}-v_{i}^{c}\right)(d t, d x)
\end{aligned}
$$

Also notice that if we define

$$
\begin{aligned}
\sigma_{i}^{*}: & =b_{j}\left(t, T_{i}, c\right)-b_{j}\left(t, T_{i+1}, c\right)=\int_{T_{i}}^{T_{i+1}} \sigma_{i}(t, s, c) d s \\
\delta_{i}^{*}: & =h\left(t, T_{i}, x\right)-h\left(t, T_{i+1}, x\right)=\int_{T_{i}}^{T_{i+1}} \delta_{i}(t, s, x) d s
\end{aligned}
$$

we finally obtain the expression

$$
\begin{aligned}
\frac{\delta}{1+\delta L\left(t, T_{i}, c\right)} d L\left(t, T_{i}, c\right)= & \sigma_{i}^{*} \sum_{\eta(t) \leq j \leq i} \sigma_{j}^{*} d t+\sigma_{i}^{*} d W_{t} \\
& +\int_{\mathbb{R}^{r}}\left(e^{\delta_{i}^{*}}-1+e^{\sum_{\eta(t) \leq j \leq i} \delta_{j}^{*}}\left(e^{\delta_{i+1}^{*}}-1\right)\right) v_{i}^{c}(d t, d x) \\
& +\int_{\mathbb{R}^{r}}\left(e^{\delta_{i}^{*}}-1\right)\left(\mu_{i}^{c}-v_{i}^{c}\right)(d t, d x)
\end{aligned}
$$

The next theorem shows the drift constraints in order to obtain the risk-neutral measure. It is an adaptation of Proposition 3.15 in Bjork et al. (1997), Theorem 3.1. of Glasserman and Kou (2003) or Eberlein and Özkan (2005).

Theorem 102 For any $i=0,1, \ldots, n$ let $\sigma_{i}($.$) be a bounded \mathbb{R}^{d}$ valued function and $\delta_{i}(t, x, s): \mathbb{R}_{+} \times \mathbb{R}^{r} \rightarrow$ $(-1, \infty)$ be deterministic. Then our default-free LIBOR model

$$
\frac{d L\left(t, T_{i}, c\right)}{L\left(t-, T_{i}, c\right)}=\alpha_{i}\left(t, T_{i}, c\right) d t+\sigma_{i}\left(t, T_{i}, c\right) d W_{t}+\int_{\mathbb{R}^{r}} \delta\left(t, x, T_{i}\right)\left(\mu_{i}^{c}-v_{i}^{c}\right)(d t, d x)
$$

is arbitrage-free if the drift takes the following expression

$$
\begin{aligned}
\alpha_{i}\left(t, T_{i}, c\right)= & \sum_{j=\eta(t)}^{i} \frac{\delta \sigma_{j}\left(t, T_{j}, c\right) \sigma_{i}\left(t, T_{i}, c\right) L\left(t-, T_{j}, c\right)}{1+\delta L\left(t-, T_{j}, c\right)} \\
& +\int_{\mathbb{R}^{r}} \delta_{i}\left(t, x, T_{i}\right)\left(1-\prod_{j=\eta(t)}^{i} \frac{1+\delta L\left(t-, T_{j}, c\right)}{1+\delta L\left(t-, T_{j}, c\right)\left(1+\delta_{j}\left(t, x, T_{i}\right)\right)}\right) v_{i}^{c}(t, d x)
\end{aligned}
$$

Proof. For the sake of clarity, let us skip the notation related with the conditional credit rating. 
Therefore, we know that

$$
\begin{aligned}
\frac{\delta}{1+\delta L\left(t, T_{i}\right)} d L\left(t, T_{i}\right)= & \sigma_{i}^{*} \sum_{\eta(t) \leq j \leq i} \sigma_{j}^{*} d t+\sigma_{i}^{*} d W_{u} \\
& +\int_{\mathbb{R}^{r}}\left(e^{\delta_{i}^{*}}-1+e^{\sum_{\eta(t) \leq j \leq i} \delta_{j}^{*}}\left(e^{\delta_{i+1}^{*}}-1\right)\right) v_{i}(d t, d x) \\
& +\int_{\mathbb{R}^{r}}\left(e^{\delta_{i}^{*}}-1\right)\left(\mu_{i}-v_{i}\right)(d t, d x)
\end{aligned}
$$

Let us define

$$
\begin{aligned}
\sigma_{i}^{*} & =\int_{T_{i}}^{T_{i}+\delta} \sigma_{i}(t, s) d s:=\frac{\delta \sigma_{i}(t) L\left(t, T_{i}\right)}{1+\delta L\left(t, T_{i}\right)} \\
\delta_{i}^{*} & =\int_{T_{i}}^{T_{i}+\delta} \delta_{i}(t, x, s) d s:=\log \left(\frac{1+\delta L\left(t, T_{i}\right)\left(1+\delta_{i}(t, x)\right)}{1+\delta L\left(t-, T_{i}\right)}\right)
\end{aligned}
$$

so that

$$
\sum_{\eta(t) \leq j \leq i} \sigma_{j}^{*}=\sum_{\eta(t) \leq j \leq i} \frac{\delta \sigma_{j}(t) L\left(t, T_{i}\right)}{1+\delta L\left(t, T_{i}\right)}
$$

and

$$
\begin{aligned}
e^{\delta_{i}^{*}} & =\frac{1+\delta L\left(t-, T_{i}\right)\left(1+\delta_{i}(t, x)\right)}{1+\delta L\left(t-, T_{i}\right)} \\
& =1+\frac{\delta L\left(t-, T_{i}\right) \delta_{i}(t, x)}{1+\delta L\left(t-, T_{i}\right)} \\
e^{\sum_{\eta(t) \leq j \leq i} \delta_{j}^{*}} & =\prod_{j=\eta(t)}^{i} e^{-\int_{T_{j}}^{T_{j}+\delta} \delta_{j}(t, x, s) d s} \\
& =\prod_{j=\eta(t)}^{i} \frac{1+\delta L\left(t-, T_{i}\right)}{1+\delta L\left(t-, T_{i}\right)\left(1+\delta_{j}(t, x)\right)}
\end{aligned}
$$

Hence, we have that the first part of the stochastic differential equation can be written as:

$$
\begin{aligned}
\frac{1+\delta L\left(t-, T_{i}\right)}{\delta} \sigma_{i}^{*} \sum_{\eta(t) \leq j \leq i} \sigma_{j}^{*} d t & =\frac{1+\delta L\left(t-, T_{i}\right)}{\delta} \frac{\delta \sigma_{i}(t) L\left(t-, T_{i}\right)}{1+\delta L\left(t-, T_{i}\right)} \sum_{\eta(t) \leq j \leq i} \frac{\delta \sigma_{j}(t) L\left(t-, T_{i}\right)}{1+\delta L\left(t-, T_{i}\right)} d t \\
& =L\left(t-, T_{i}\right) \sum_{\eta(t) \leq j \leq i} \frac{\delta \sigma_{j}(t) \sigma_{i}(t) L\left(t-, T_{i}\right)}{1+\delta L\left(t-, T_{i}\right)} d t
\end{aligned}
$$

and working in the second part, we obtain

$$
\begin{aligned}
\frac{1+\delta L\left(t-, T_{i}\right)}{\delta} \sigma_{i}^{*} d W_{t} & =\frac{1+\delta L\left(t-, T_{i}\right)}{\delta} \frac{\delta \sigma_{i}(t) L\left(t-, T_{i}\right)}{1+\delta L\left(t-, T_{i}\right)} d W_{t} \\
& =\sigma_{i}(t) L\left(t-, T_{i}\right) d W_{t}
\end{aligned}
$$


the fourth part will be

$$
\begin{aligned}
& \frac{1+\delta L\left(t-, T_{i}\right)}{\delta} \int_{\mathbb{R}^{r}}\left(e^{\delta_{i}^{*}}-1\right)\left(\mu_{i}-v_{i}\right)(d t, d x) \\
= & \frac{1+\delta L\left(t-, T_{i}\right)}{\delta} \int_{\mathbb{R}^{r}}\left(\frac{\delta L\left(t-, T_{i}\right) \delta_{i}(t, x)}{1+\delta L\left(t-, T_{i}\right)}\right)\left(\mu_{i}-v_{i}\right)(d t, d x) \\
= & L\left(t-, T_{i}\right) \int_{\mathbb{R}^{r}} \delta_{i}(t, x)\left(\mu_{i}-v_{i}\right)(d t, d x)
\end{aligned}
$$

and using the last result, the third part can be re-written as

$$
\begin{aligned}
& \frac{1+\delta L\left(t-, T_{i}\right)}{\delta} \int_{\mathbb{R}^{r}}\left(e^{\delta_{i}^{*}}-1+e^{\sum_{\eta(t) \leq j \leq i-1} \delta_{j}^{*}}\left(e^{\delta_{i}^{*}}-1\right)\right) v_{i}(d t, d x) \\
= & \frac{1+\delta L\left(t-, T_{i}\right)}{\delta} \int_{\mathbb{R}^{r}}\left(\frac{\delta L\left(t-, T_{i}\right) \delta_{i}(t, x)}{1+\delta L\left(t-, T_{i}\right)}\left(1-\prod_{j=\eta(t)}^{i} \frac{1+\delta L\left(t-, T_{i}\right)}{1+\delta L\left(t-, T_{i}\right)\left(1+\delta_{j}(t, x)\right)}\right)\right) v_{i}(d t, d x) \\
= & L\left(t-, T_{i}\right) \int_{\mathbb{R}^{r}} \delta_{i}(t, x)\left(1-\prod_{j=\eta(t)}^{i} \frac{1+\delta L\left(t-, T_{i}\right)}{1+\delta L\left(t-, T_{i}\right)\left(1+\delta_{j}(t, x)\right)}\right) v_{i}(d t, d x)
\end{aligned}
$$

Finally, combining the last four results, we conclude that

$$
\begin{aligned}
\frac{d L\left(t, T_{i}\right)}{L\left(t-, T_{i}\right)}= & \left(\sum_{\eta(t) \leq j \leq i} \frac{\delta \sigma_{j}(t) \sigma_{i}(t) L\left(t-, T_{i}\right)}{1+\delta L\left(t-, T_{i}\right)}+\right. \\
& \left.\int_{\mathbb{R}^{r}} \delta_{i}(t, x)\left(1-\prod_{j=\eta(t)}^{i} \frac{1+\delta L\left(t-, T_{i}\right)}{1+\delta L\left(t-, T_{i}\right)\left(1+\delta_{j}(t, x)\right)}\right) v_{i}(t, d x)\right) d t \\
& +\sigma_{i}(t) d W_{t} \\
& +\int_{\mathbb{R}^{r}} \delta_{i}(t, x)\left(\mu_{i}-v_{i}\right)(d t, d x)
\end{aligned}
$$




\subsection{LIBOR additive model calibration to swaptions markets}

To have a robust and efficient algorithm is a central topic in the successful implementation of a model. The amount of reality that the model can collect is not only related with the sort of process that drives the model but also with the calibration methodology that you use to substract the information from the market prices. Therefore the calibration of the financial models has become an important topic in financial engineering because of the need to price increasingly complex options in a consistent way with prices of standard instruments liquidly traded in the market. The selection of the calibration methodology is a crucial step in the correct implementation of the model.

Let us recall that the sort of non-homogeneous processes studied in Chapter 1 can be divided into two categories: in the first category, called jump-diffusion models, the 'normal' evolution of prices or interest rates is given by diffusion processes, punctuated by jumps at random intervals. Here the jumps represent rare events (crashes and large breakdowns). Such an evolution can be represented by modelling the interest rates as a non-homogeneous Lévy process with a nonzero Gaussian component and a jump part, which is a compound Poisson process with finitely many jumps in every time interval. In these models, the dynamic structure of the process is easy to understand and describe, since the distribution of jumps sizes is known. Examples of such models are the Merton (1976) jump-diffusion model with Gaussian jumps and Kou (2002) model with double exponential jumps. They are easy to simulate and efficient Monte Carlo methods for pricing path-dependent options can be used. Models of this type perform quite well for the purposes of implied volatility smile interpolation. However they rarely lead to closed-form densities: statistical estimation and computation of moments or quantiles may be difficult.

The second category consists of models with an infinite number of jumps in every interval, which we will call infinity activity models. In these models one does not need to introduce a Brownian motion as a component in the model since the dynamics of the jumps is already rich enough to generate nontrivial small time behavior (see Carr, Geman, Madan and Yor (2002)) and it has been argued in Madan (2001) or Geman (2002) that such models give a more realistic description of the price process at various time scales. In addition, many models from this class can be constructed via Brownian subordination which gives them additional analytical tractability compared to jump-diffusion models.

But independently of the category of the process that drives our model, the practitioner has to guarantee two properties in the calibration process:

- first, the calibration solution has to be unique and global and this sort of results are only possible if the calibration problem is a convex problem,

- and second, the calibration process has to provide an indication of the sensitiveness of our calibration to market movements (robustness). It is usually given as the dual solution of the convex problem.

Basically, the main goal in this section is to propose a convex problem that provides a unique and global solution for the primal and a solution in the dual problem that will be an indicator of robustness. Our proposal is related with how to calibrate and work with the first category of models using convex programming methods. Notice that as a direct implication of the Lévy-Itô decomposition is that every Lévy process is a combination of a Brownian motion with drift and a possibly infinite sum of independent compound Poisson process (see Theorem 9 in section 1.1.3). This also means that every LIBOR additive process can be approximated with arbitrary precision by a sequence of jump-diffusion processes (Theorems 40 and 41 in section 1.6), that is by a sum of a sequence of Brownian motions with drifts and a sequence of compound Poisson process, a point which is useful not only in theory but also from the practitioner point of view.

Therefore this section contains three different parts: 
- The first part (section 3.3.1) gives basically a brief introduction about what we called two-steps calibration

- The second part (section 3.3.2) is devoted to the first-step calibration or calibration to the continuous part of the model. Using the at-the-money swaption volatilities, we propose a convex methodology to obtain the term structure of instantaneous volatilities and covariances from the market data.

- In the third part (section 3.3.3), our aim is to propose the second-step calibration as an inverse problem to calibrate the sequence of Lévy measures according to the information given by the smile/skew in the swaption market.

\subsubsection{An Introduction to the two-steps calibration for the LIBOR additive process}

From the beginning of the present thesis, the reader would wonder to know the advantages of this new stochastic process, the LIBOR additive process, in the interest rates modelling. This subsection will show briefly the main reason: the double-calibration of this process against a non-homogeneous swaption market with volatility smiles. Basically the idea is simple: according to the Lévy-Itô decomposition of the LIBOR additive process (Theorems 40 and 41 in section 1.6), we can define the calibration problem as an inverse problem of a sequence of triplets that completely characterize the entire process.

Let us consider the following discretization in $\left[0, T^{\star}\right], 0<T_{1}<\ldots<T_{n}=T^{\star}$. Let us define the price of a payer swaption at time $t$ as $P S\left(t, T_{i}, T_{j}, \sigma_{i, j}^{\star}, K_{i, j}^{h}\right)$ as a call-option to get into a swap that begins in $T_{i}$ and finish in $T_{j}$ with the swap rate $S\left(t, T_{i}, T_{j}\right)$ (underlying of the option) with strike $K_{i, j}$ and $0<i \leq j \leq n$, and where $\sigma_{i, j}^{\star}$ is the Black (1976) cumulative variance of swaption on $S\left(t, T_{i}, T_{j}\right)$ for the mentioned strike $K_{i, j}$ quoted in the swaption market.

Proposition 103 The general calibration problem at the moment $t$ can be written as an inverse problem defined as

$\left(\gamma_{i}, A_{i}, v_{i}\right)_{i \in\{1, \ldots, n\}}=\arg \inf \sum_{h=-m}^{m} \sum_{i=1}^{n} \sum_{j=i+1}^{n}\left[\omega_{i j}^{h}\left\|P S^{\sigma}\left(t, T_{i}, T_{j}, A_{i}, v_{i}, K_{i, j}^{h}\right)-P S^{M}\left(t, T_{i}, T_{j}, \sigma_{i, j}^{\star}, K_{i, j}^{h}\right)\right\|\right]$

where, on the right part of the equality, we have denoted as $P S^{\sigma}\left(t, T_{i}, T_{j}, A_{i}, v_{i}, K_{i, j}^{h}\right)$ the theoretical payer swaption price in $t$, given by our model, and defined as the value of an option with maturity $T_{i}$ that gives to the holder the right to get into a forward payer swap between $T_{i}$ and $T_{j}$ settled in arrears, with $0<i \leq j \leq n$, and $P S^{M}\left(t, T_{i}, T_{j}, \sigma_{i, j}^{\star}, K_{i, j}^{h}\right)$ as the market value of a payer swaption in $t$, priced using Black-76 model, with strike $K_{i, j}^{h}$, where $h \in \mathbb{N}$ such that if $h=0$ then $F S_{t}\left(K_{i, j}^{0}\right)=0$ (at-the-money case), and market volatility $\sigma_{i, j}^{\star}$ such that

$$
\left\{\begin{array}{l}
P S^{\sigma}\left(t, T_{i}, T_{j}, A_{i}, v_{i}, K_{i, j}^{h}\right)=\mathbb{E}_{\mathbb{P}^{*}}\left\{B\left(t, T_{i}\right)\left(F S_{T_{i}}\left(K_{i, j}^{h}\right)\right)^{+} \mid \mathcal{F}_{t}\right\} \\
P S^{M}\left(t, T_{i}, T_{j}, \sigma_{i, j}^{\star}, K_{i, j}^{h}\right)=\operatorname{Black}\left(t, T_{i}, T_{j}, \sigma_{i, j}^{\star}, K_{i, j}^{h}\right) \sum_{i \leq j} B\left(t, T_{i}\right) \delta_{i}
\end{array}\right.
$$

and $\left(\gamma_{i}, A_{i}, v_{i}\right)_{i=1, \ldots, n}$ is the sequence of triplets consisting of

$$
\left\{\begin{array}{l}
\gamma_{i} \in \mathbb{R}^{d} \\
A_{i}=\left(\sigma_{i j}\right), \text { a } d \times d \text { symmetric non-negative matrix, with } d=n-i+1 \\
v_{i} \text { a positive measure on } \mathbb{R}^{d} \backslash\{0\} \text { with } \int_{\mathbb{R}^{d}}\left(|g|^{2} \wedge 1\right) v(d g)<\infty
\end{array}\right.
$$

Proof. Notice that the basic underlying that is moved by the LIBOR additive process is the forward LIBOR rates, according to the model specified in this chapter. Let us consider the usual 
payer swaption definition that we have to price it using our model is

$$
\begin{aligned}
P S^{\sigma}\left(t, T_{i}, T_{j}, A_{i}, v_{i}, K_{i, j}^{h}\right) & =\mathbb{E}_{\mathbb{P} *}\left\{B\left(t, T_{i}\right)\left(F S_{T_{i}}\left(K_{i, j}^{h}\right)\right)^{+} \mid \mathcal{F}_{t}\right\} \\
& =\mathbb{E}_{\mathbb{P} *}\left\{B\left(t, T_{i}\right)\left(\mathbb{E}_{\mathbb{P} *}\left(\sum_{\beta=i+1}^{j} B\left(T_{i}, T_{\beta}\right)\left(L\left(t, T_{\beta-1}\right)-K_{i, j}^{h}\right) \delta_{j} \mid \mathcal{F}_{T_{i}}\right)\right)^{+} \mid \mathcal{F}_{t}\right\}
\end{aligned}
$$

that after simple manipulations and using assumptions in 3.1.3 related with swaps and swaptions, then this yields, as expected

$$
P S^{\sigma}\left(t, T_{i}, T_{j}, \sigma_{i, j}^{\star}, K_{i, j}^{h}\right)=\mathbb{E}_{\mathbb{P} \star}\left\{B\left(t, T_{i}\right)\left(1-\sum_{\beta=i+1}^{j} c_{\beta} B\left(T_{i}, T_{\beta}\right)\right)^{+} \mid \mathcal{F}_{t}\right\}
$$

or in other words, the payer swaption may also be seen as a put option on a coupon-bearing bond, where $c_{\beta}=K_{i, \beta}^{h} \delta_{\beta}$ when $\beta=i, \ldots, j-1$ and $c_{j}=1+K_{i, j}^{h} \delta_{j}$ when $\beta=j$.

Additionally, notice that using the same approximation that has already been employed by Brace, Gatarek and Musiela (1997) we can write the payer swaption value as a function of the LIBOR rates as

$$
\begin{aligned}
P S^{\sigma}\left(t, T_{i}, T_{j}, \sigma_{i, j}^{\star}, K_{i, j}^{h}\right) & =B\left(t, T_{i}\right) \mathbb{E}_{\mathbb{P}^{\star}}\left\{\left(1-\sum_{\beta=i+1}^{j} c_{\beta} B\left(T_{i}, T_{\beta}\right)\right)^{+} \mid \mathcal{F}_{t}\right\} \\
& =B\left(t, T_{i}\right) \mathbb{E}_{\mathbb{P}^{\star}}\left\{\left.\left(1-\sum_{\beta=i+1}^{j} c_{\beta} \prod_{l=i+1}^{\beta-1}\left(1+\delta_{\beta} L\left(T_{i}, T_{l}\right)\right)^{-1}\right)^{+}\right|^{\mathcal{F}_{t}}\right\} \\
& =B\left(t, T^{\star}\right) \mathbb{E}_{\mathbb{P}^{\star}}\left\{\prod_{l=\alpha+1}^{n-1}\left(1+\delta_{j} L\left(T_{i}, T_{l}\right)\right)\left(1-\sum_{\beta=i+1}^{j} c_{\beta} \prod_{l=i+1}^{\beta-1}\left(1+\delta_{\beta} L\left(T_{i}, T_{l}\right)\right)^{-1}\right)^{+} \mathcal{F}_{t}\right\} \\
& =B\left(t, T^{\star}\right) \mathbb{E}_{\mathbb{P}^{\star}}\left\{\left(-\left.\sum_{\beta=i}^{j}\left(c_{\beta} \prod_{l=j}^{n-1}\left(1+\delta_{\beta} L\left(T_{i}, T_{l}\right)\right)\right)^{+}\right|^{\mathcal{F}_{t}}\right\}\right.
\end{aligned}
$$

where the dynamics of the forward LIBOR rates driven by a LIBOR additive process, is specified as

$$
L\left(t, T_{k}\right)=L\left(0, T_{k}\right) \exp \left(\int_{0}^{t} \lambda_{j}\left(s, T_{k}\right) d G_{s}^{T^{\star}}\right)
$$

We have proved in Chapter 1 (Section 1.3) that this process is uniquely determined in law by its sequence of triplets $\left(\gamma_{i}, A_{i}, v_{i}\right)_{i \in\{1, \ldots, n\}}$ consisting of

$$
\left\{\begin{array}{l}
\gamma_{i} \in \mathbb{R}^{d} \\
A_{i}=\left(\sigma_{i j}\right), \text { a } d \times d \text { symmetric non-negative matrix, with } d=n-i+1 \\
\nu_{i} \text { a positive measure on } \mathbb{R}^{d} \backslash\{0\} \text { with } \int_{\mathbb{R}^{d}}\left(|g|^{2} \wedge 1\right) v(d g)<\infty
\end{array}\right.
$$

Notice that using directly the independence property between the continuous and the jump part, implicit in the Lévy-Itô theorem (theorems 40 and 41 in section 1.6.), we can prove directly the following proposition,

Proposition 104 The inverse problem (3.12) can be split in two different and independent inverse prob- 
lems

(1) $A_{i}=\arg \inf \sum_{j=i+1}^{n}\left[\omega_{i j}^{0}\left\|P S^{\sigma}\left(t, T_{i}, T_{j}, A_{i}, v_{i}, K_{i, j}^{0}\right)-P S^{M}\left(t, T_{i}, T_{j}, \sigma_{i, j}^{\star}, K_{i, j}^{h}\right)\right\|\right]$
(2) $v_{i}=\arg \inf \sum_{h=-m}^{m} \sum_{j=i+1}^{n}\left[\omega_{i j}^{h}\left\|P S^{\sigma}\left(t, T_{i}, T_{j}, A_{i}, v_{i}, K_{i, j}^{h}\right)-P S^{M}\left(t, T_{i}, T_{j}, \sigma_{i, j}^{\star}, K_{i, j}^{h}\right)\right\|\right]$

Therefore we can calibrate this sequence of triplets against the market separately: on one hand, the continuous part calibration will be achieved using at-the-money swaption volatilities, and on the other hand, the sequence of Lévy measures will be estimated using in/out -of -the-money swaption smiles for different maturities.

The main goal of this two-step calibration is to collect as much information as we can from the market prices, in order to simulate the most realistic possible scenarios, but also provide robust and global solutions to the calibration problem. The methodology behind this calibration procedure is not new, several authors have introduced these ideas in the continuous process framework or in stock market. However, our approach has two improvements.

- In the first-step, we introduce some relevant changes in the SDP problem that guarantee not only the convexity but also the stability of the solution, something essential to achieve the correct simulation in the full-rank LIBOR additive model.

- And with the second-step of the calibration, we introduce the information given by the smile in the swaption market directly in the sequence of the Lévy measures, creating a direct link between jumps and smile.

\subsubsection{First-step: semidefinite programming to calibrate the continuous Mar- ket model}

Obviously, a robust and efficient calibration algorithm is a central element in the successful implementation of a derivatives pricing model, independently if the model is driven by semimartingales or directly by a Brownian motion. Recent developments in interest rates modelling have led to a form of technological asymmetry on this topic. The theoretical performance of the usual continuous models such as the already mentioned (Heath, Jarrow and Morton (HJM) (1992) or the LIBOR Market Model of Interest Rates by Brace, Gaterek and Musiela(BGM)(1997)) allows a very flexible modelling and pricing of the basic interest rate options (caps and swaptions) at-the-money. However, due to the inefficiency and instability of the calibration procedure, only a small part of the market covariance information (that could theoretically be accounted for in the model) is actually exploited.

To be precise, the most common techniques to calibrate the continuous part of the model (see for example Longstaff, Santa-Clara and Schwartz (2000), Rebonato (2000) or Brigo and Mercurio (2002)) are methods clearly limited. Usually it is necessary to substitute a statistical estimate to the market information on the forward LIBOR correlation matrix because the numerical complexity and instability of the calibration process makes it impossible to calibrate a full market covariance matrix. As a direct consequence, these calibration algorithms fail in one of their primary mission: they are very poor market risk visualization tools.

The forward rates covariance matrix plays an increasingly important role in exotic interest rate derivatives modelling and there is a need for a calibration algorithm that allows the retrieval of a maximum amount of covariance information from the market. As far as we know, Brace and Womersley (2000) and d'Aspremont (2003) are the only that propose a methodology to calibrate a multivariate LIBOR market model without assuming any 'a priory' structure to the covariance matrix, based on semidefinite programming, allowing at the same time robust and global solutions. They showed how semidefinite programming based calibration methods provide integrated calibration and risk-management results with 
guaranteed numerical performance, the dual program having a very natural interpretation in terms of hedging instruments and sensitivity.

Our main goal in this subsection, is to provide an extension of Brace and Womersley (2000) or d'Aspremont (2003) methodologies that has the goal of solving the calibration problem for the continuous part of our model, improving the stability and robustness of the Brace and Womersley (2000) or d'Aspremont (2003) solutions. Basically, our proposal is based on a relevant change in the objective function that allows us to skip the dependence of the solutions with respect to how to formulate the objective function.

\section{Introduction to the swaption calibration problem}

Let us study the swaption market under the Brace, Gaterek and Musiela model. Let us define the swap rate as the fixed rate that zeroes the present value of a set of periodical exchanges of fixed against floating coupons on a LIBOR rate of given maturity at futures dates. Denoting by $S\left(t, T_{1}, T_{n}\right)$ a forward swap rate at time $t$ for an interest rate swap with first reset at $T_{1}$ and exchanging payments at $T_{1}, \ldots, T_{n}$. It is clear that it is stochastic and under the appropriate measure swap measure $\mathbb{Q}^{1, n}$ we can assume a lognormal dynamics for the continuous part of the swap dynamics

$$
d \tilde{S}\left(t, T_{1}, T_{n}\right)=S\left(t, T_{1}, T_{n}\right) \sigma_{1, n}(t) d W_{t}^{\mathbb{Q}^{1, n}}
$$

However, analytical approximations are available for swaptions in the LIBOR Market model framework. Indeed, Brace, Dun and Barton (1999) suggest to adopt the LIBOR forward market as the central model for the two markets, mainly for its mathematical tractability. We will stick to their suggestion, also because of the fact that forward rates are somehow more natural and more representative coordinates of the yield-curve than swap rates.

To introduce the formula we will use in the following, note that a crucial role in the swap market model is played by the Black swap volatility $\sigma_{1, n}^{\star}\left(T_{1}\right)$ entering Black's formula for swaptions, expressed by

$$
\begin{aligned}
\sigma_{1, n}^{\star}\left(T_{1}\right) & :=\frac{1}{T_{1}} \int_{0}^{T_{1}}\left(\sigma_{1, n}(t)\right)^{2} d t \\
& =\frac{1}{T_{1}} \int_{0}^{T_{1}}\left(d \ln \tilde{S}\left(t, T_{1}, T_{n}\right)\right)\left(d \ln \tilde{S}\left(t, T_{1}, T_{n}\right)\right)
\end{aligned}
$$

Notice that if we choose the LIBOR Market Model as a central model, we must resort to different pricing techniques. It is possible to price swaptions with a Monte Carlo simulation, by simulating the forward rates involved in the payoff through a discretization of the dynamics presented above, so as to obtain the relevant zero coupon bonds and the forward swap rate. In fact, recall that we can write the forward-swap as a basket of forwards (see Rebonato (1998))

$$
S\left(t, T_{1}, T_{n}\right)=\sum_{i=1}^{n} \omega_{i}(t) F\left(t, T_{i}\right)
$$

where $\omega_{i}$ are the weights (with an explicit expression) such that $0 \leq \omega_{i} \leq 1$ (in fact, they are always 
positive, monotone and sum to one) such that

$$
\begin{aligned}
\omega_{i}(t) & =\frac{\tau_{i} B\left(t, T_{1}, T_{i}\right)}{\sum_{i=2}^{n} \tau_{i} B\left(t, T_{1}, T_{i}\right)} \\
= & \frac{\tau_{i} \prod_{j=2}^{i} \frac{1}{1+\tau_{j} F\left(t, T_{j}\right)}}{\sum_{i=2}^{n} \tau_{i} \prod_{j=2}^{i} \frac{1}{1+\tau_{j} F\left(t, T_{j}\right)}}
\end{aligned}
$$

One can compute, under a number of approximations, based on "partially freezing the drift" and on "collapsing all measures" in the original dynamics, an analogous quantity $\sigma_{1, n}^{\star}\left(T_{1}\right)$ in the LMM.

We present here one of the simplest ways to deduce this formula based on a similar setting, appeared earlier for example in Rebonato (1998), and tested against Monte Carlo simulations for instance in Brigo and Mercurio (2001). Such approximated formulae, are easily obtained, first, freezing the weight's at time 0 , so as to obtain

$$
S\left(t, T_{1}, T_{n}\right)=\sum_{i=1}^{n} \omega_{i}(0) F\left(t, T_{i}\right) .
$$

Notice that this approximation is justified by the fact that the variability of the $\omega$ 's is much smaller than the variability of the forward rates. This can be tested both historically and through simulations of the forward rates via Monte Carlo methods (see Brigo and Mercurio (2001)).

Then, let us differentiate both sides and we obtain

$$
\begin{aligned}
d \tilde{S}\left(t, T_{1}, T_{n}\right) & \approx \sum_{i=1}^{n} \omega_{i}(0) d F\left(t, T_{i}\right) \\
& =(\ldots) d t+\sum_{i=1}^{n} \omega_{i}(0) \sigma_{i}(t) F\left(t, T_{i}\right) d W_{i}(t)
\end{aligned}
$$

under any of the forward-adjusted measures, and compute the quadratic variation

$$
d \tilde{S}\left(t, T_{1}, T_{n}\right) d \tilde{S}\left(t, T_{1}, T_{n}\right) \approx \sum_{i, j=1}^{n} \omega_{i}(0) \omega_{j}(0) F\left(t, T_{i}\right) F\left(t, T_{j}\right) \rho_{i, j} \sigma_{i}(t) \sigma_{j}(t) d t
$$

and the percentage quadratic variation is

$$
\begin{aligned}
\left(\frac{d \tilde{S}\left(t, T_{1}, T_{n}\right)}{S\left(t, T_{1}, T_{n}\right)}\right)\left(\frac{d \tilde{S}\left(t, T_{1}, T_{n}\right)}{S\left(t, T_{1}, T_{n}\right)}\right) & =\left(d \ln \tilde{S}\left(t, T_{1}, T_{n}\right)\right)\left(d \ln \tilde{S}\left(t, T_{1}, T_{n}\right)\right) \\
& \approx \frac{1}{T_{1}} \frac{\sum_{i, j=1}^{n} \omega_{i}(0) \omega_{j}(0) F\left(t, T_{i}\right) F\left(t, T_{j}\right) \rho_{i, j} \sigma_{i}(t) \sigma_{j}(t)}{S\left(t, T_{1}, T_{n}\right)^{2}} d t
\end{aligned}
$$

Now we can assume that freezing all forward rates in the above formula to their time-zero value

$$
\left(d \ln \tilde{S}\left(t, T_{1}, T_{n}\right)\right)\left(d \ln \tilde{S}\left(t, T_{1}, T_{n}\right)\right) \approx \frac{1}{T_{1}} \sum_{i, j=1}^{n} \frac{\omega_{i}(0) \omega_{j}(0) F_{B}\left(0, T_{i}\right) F_{B}\left(0, T_{j}\right)}{S\left(t, T_{1}, T_{n}\right)^{2}} \rho_{i, j} \sigma_{i}(t) \sigma_{j}(t) d t
$$


and finally we can obtain the expression for $\left(\sigma_{1, n}^{\star}\left(T_{1}\right)\right)^{2}$ such that

$$
\begin{aligned}
& \int_{0}^{T_{1}}\left(d \ln \tilde{S}\left(t, T_{1}, T_{n}\right)\right)\left(d \ln \tilde{S}\left(t, T_{1}, T_{n}\right)\right) \\
\approx & \frac{1}{T_{1}} \sum_{i, j=1}^{n} \frac{\omega_{i}(0) \omega_{j}(0) F\left(0, T_{i}\right) F\left(0, T_{j}\right)}{S\left(t, T_{1}, T_{n}\right)^{2}} \rho_{i, j} \int_{0}^{T_{1}} \sigma_{i}(t) \sigma_{j}(t) d t:=\left(\sigma_{1, n}^{\star}\left(T_{1}\right)\right)^{2}
\end{aligned}
$$

and this result proves the following proposition, given by Rebonato (1998)

Proposition 105 The LIBOR market model (squared) swaption volatility can be approximated by

$$
\left(\sigma_{1, n}^{\star}\left(T_{1}\right)\right)^{2} T_{1}=\sum_{i, j=1}^{n} \frac{\omega_{i}(0) \omega_{j}(0) F\left(0, T_{i}\right) F\left(0, T_{j}\right)}{S\left(t, T_{1}, T_{n}\right)^{2}} \rho_{i, j} \int_{0}^{T_{1}} \sigma_{i}(t) \sigma_{j}(t) d t
$$

The quantity $\sigma_{1, n}^{\star}\left(T_{1}\right)$ can be used as a proxy for the Black volatility of the swap rate $S\left(t, T_{1}, T_{n}\right)$. Putting this quantity in Black's formula for swaption allows one to compute approximated swaptions prices with the LIBOR market model (continuous part). Notice this result is obtained under a number of assumptions, and at first one would imagine its quality to be rather poor. However, it turns out that the approximation is very accurate as also pointed out by Brace, Dun and Barton (1998) and Brigo and Mercurio (2001).

\section{The calibration problem}

In this subsection we introduce the practical implementation of the calibration program using the swaption pricing approximation detailed above. Now, for the sake of reality, let us introduce a change of the notation.

We suppose that the calibration data set is made of $m$ swaptions with option maturity $T_{\alpha}$ written on swaps of maturity $T_{\beta}-T_{\alpha}$ for $\alpha<\beta$, where $\alpha, \beta \in \mathbb{N}^{+}$, and $T_{\alpha}, T_{\beta} \in\left\{T_{1}, \ldots, T_{n}\right\}$ with $n \in \mathbb{N}^{+}$, with market volatility given by $\sigma_{\alpha, \beta}^{\star}\left(T_{\alpha}\right)$. Notice that in our non-homogeneous case where $\sigma_{i}(t)$ is of the form $\sigma\left(t, T_{i}, T_{i+1}\right)$ for any $i=1, \ldots, n$, with $t<T_{i}$ and piecewise constant on intervals of size $\tau=T_{i+1}-T_{i}$.

Therefore, the expression of the market cumulative variance, according to Rebonato's formula (3.14), can be expressed as

$$
\begin{aligned}
\left(\sigma_{\alpha, \beta}^{\star}\left(T_{\alpha}\right)\right)^{2} T_{\alpha} & =\int_{0}^{T_{\alpha}} \sum_{i, j=\alpha}^{\beta} \frac{\omega_{i}(0) F\left(0, T_{i}\right)}{S\left(0, T_{\alpha}, T_{\beta}\right)} \frac{\omega_{j}(0) F\left(0, T_{j}\right)}{S\left(0, T_{\alpha}, T_{\beta}\right)} \rho_{i, j} \sigma_{i}(s) \sigma_{j}(s) d s \\
& =\int_{0}^{T_{\alpha}} \sum_{i, j=\alpha}^{\beta} \hat{\omega}_{i}(0) \hat{\omega}_{j}(0) \cdot \sigma_{i, j}(s) \cdot d s \\
& =\int_{0}^{T_{\alpha}} \operatorname{Tr}\left(\Omega_{\alpha, \beta} A_{s}\right) d s \\
& =\operatorname{Tr}\left(\Omega_{\alpha, \beta} \int_{0}^{T_{\alpha}} A_{s} d s\right)
\end{aligned}
$$

where $A_{s}=\left(\sigma_{i, j}(s)\right)_{i, j \in[\alpha, \beta]}=\left(\rho_{i, j} \sigma_{i}(s) \sigma_{j}(s)\right)_{i, j \in[\alpha, \beta]}$ and $\Omega_{\alpha, \beta}=\hat{\omega}(0) \hat{\omega}(0)^{\prime}=\left(\hat{\omega}_{i}(0) \hat{\omega}_{j}(0)\right)_{i, j \in[\alpha, \beta]}$.

These conditions show that the cumulative market variance of a particular swaption can be written as the linear function of the forward covariance matrix, or equivalently we can say that here swaptions 
are priced as basket options with constant coefficients. As detailed in Brace and Womersley (2000) or d'Aspremont (2003), this simple approximation creates a relative error on swaption prices of $1-2 \%$, which is well within bid-ask spreads.

On the other hand, if we want to formulate the calibration problem, this conditions has to be extended for every $\alpha=1, \ldots, n$ in order to capture all the swaptions volatilities that the market quotes. Therefore the calibration problem becomes, using the approximate swaption variance formula in (3.16):

$$
\begin{array}{cc}
\text { Find } & A \\
\text { subject to } & \operatorname{Tr}\left(\Omega_{i, n} A\right)=\left(\sigma_{i, n}^{\star}\left(T_{i}\right)\right)^{2} T_{i} \quad \text { with } i=1, \ldots, n \\
& A \succeq 0
\end{array}
$$

which is a semidefinite feasible problem in the covariance matrix $A \in \mathbb{S}^{N}$ and $\Omega_{i, n} \in \mathbb{S}^{N}$, and $\left(\sigma_{i, n}^{\star}\left(T_{i}\right)\right)^{2} T_{i} \in$ $R^{+}$is given by the swaption market as the Black (1976) cumulative variance of swaption on $S\left(0, T_{i}, T_{n}\right)$. Notice that $A$ and $\Omega$ are block diagonal matrices that represent how the different factors disappear with the time (see Brigo and Mercurio (2001) section 7.1) such that $N=n(n-1) / 2$ and $A_{i} \in \mathbb{S}^{n-(i-1)}$ in the following sense

$$
A=\left[\begin{array}{cccc}
A_{1} & 0 & \cdots & 0 \\
0 & A_{2} & \cdots & 0 \\
\vdots & \vdots & \ddots & \vdots \\
0 & 0 & \cdots & A_{n}
\end{array}\right]
$$

where $\left(A_{i}\right)_{i=1, \ldots, n}$ represents the sequence of semidefinite covariance matrices that characterize the continuous part of the LIBOR additive process.

The general form of the problem proposed by Brace and Womersley (2000) or d'Aspremont (2003) is the following:

$$
\begin{array}{cc}
\operatorname{Tr} & (C A) \\
\text { subject to } & \operatorname{Tr}\left(\Omega_{i} A\right)=\left(\sigma_{i, n}^{\star}\left(T_{i}\right)\right)^{2} T_{i} \quad \text { with } i=1, \ldots, n \\
A & \succeq 0
\end{array}
$$

however the calibration problem gives an entire set of solutions, extremely sensible to the matrix in the objective function $C \in \mathbb{S}^{N}$. That is clearly the biggest drawback in this framework. The general form of the problem that we propose to solve is the following:

Proposition 106 The general calibration problem can be written as an infinite-dimensional linear matrix inequality with the following objective function:

$$
\begin{array}{cc}
\text { find } & k, A \\
\text { subject to } & \operatorname{Tr}\left(\Omega_{i} A\right)=\left(\sigma_{i, n}^{\star}\left(T_{i}\right)\right)^{2} T_{i} \quad \text { with } i=1, \ldots, n \\
& \left\|A-A_{\text {hist }}\right\|_{F r} \leq k I_{N} \\
& A \succeq 0
\end{array}
$$

or equivalently,

$$
\begin{array}{cc}
\text { minimize } & \left\|A-A_{\text {hist }}\right\|_{F r} \\
\text { subject to } & \operatorname{Tr}\left(\Omega_{i} A\right)=\left(\sigma_{i, n}^{\star}\left(T_{i}\right)\right)^{2} T_{i} \quad \text { with } i=1, \ldots, n \\
A & \succeq 0
\end{array}
$$

which is a semidefinite feasibility problem in the covariance matrix $A \in \mathbb{S}^{N}$ where $A_{\text {hist }} \in \mathbb{S}^{N}$ is the historical covariance matrix, $k \in \mathbb{R}^{+}, \Omega_{i, n} \in \mathbb{S}^{N}$, and $\left(\sigma_{i, n}^{\star}\left(T_{i}\right)\right)^{2} T_{i} \in \mathbb{R}^{+}$is given by the swaption market as the Black (1976) cumulative variance of swaption on $S\left(0, T_{i}, T_{n}\right)$ where $T_{i}$ is the maturity of the option over a swap rate at time 0 for an interest rate swap with first reset at $T_{i}$ and exchanging payments at $T_{i}, \ldots, T_{n}$. 


\section{Primal-Dual SDP problem and Algorithm Implementation}

According to the last proposition the general form of the problem to be solved is given by:

$$
\begin{array}{cc}
\operatorname{minimize} & \left\|A-A_{\text {hist }}\right\|_{F r} \\
\text { subject to } & \operatorname{Tr}\left(\Omega_{i} A\right)=\left(\sigma_{i, n}^{\star}\left(T_{i}\right)\right)^{2} T_{i} \quad \text { with } i=1, \ldots, n \\
A & \succeq 0
\end{array}
$$

Because their feasible set is the intersection of an affine subspace with the convex cone of nonnegative vectors, the objective being linear, these programs are convex. Or in other words, their solution set is convex as the intersection of an affine subspace with the (convex) cone of positive semidefinite matrices and a particular solution can be found by choosing $A_{\text {hist }}$ and solving the corresponding semidefinite program. If the program is feasible, convexity guarantees the existence of a unique (up to degeneracy or unboundedness) optimal solution.

The first method used to solve these programs in practice was the simplex method. This algorithm works well in most cases but is known to have an exponential worst case complexity. In practice, this means that convergence of the simplex method cannot be guaranteed. Since the work of Nemirovskii and Yudin (1979) and Karmarkar (1984) however, we know that these programs can be solved in polynomial time by interior point methods and most modern solver implement both techniques. More importantly for our purposes here, the interior point methods used to prove polynomial time solvability of linear programs have been generalized to a larger class of convex problems. One of these extensions is called semidefinite programming. Nesterov and Nemirovskii (1994) showed that these programs can be solved in polynomial time. A number of efficient solvers are available to solve them, the one used in this work is called SEDUMI by Sturm (1999). In practice, a program with $n=50$ will be solved in less than a second.

Now, let us show how the dual solution to the calibration program provides a complete description of the sensitivity to changes in market condition. In fact, because the mentioned algorithms used to solve the calibration problem jointly solve the problem and its dual, the sensitivity of the calibrated covariance matrix is readily available from the dual solution to the calibration program. Notice that according to the standard form of the primal semidefinite program, we can write the following Lagrangian

$$
L(A, \lambda)=-\left\|A-A_{h i s t}\right\|_{F r}+\sum_{i=1}^{n} \lambda_{i}\left(\left(\sigma_{i, n}^{\star}\left(T_{i}\right)\right)^{2} T_{i}-\operatorname{Tr}\left(\Omega_{i} A\right)\right)
$$

and because the semidefinite cone is self-dual, we find that $L(A, \lambda)$ is bounded below in $A \succeq 0$, hence the dual semidefinite problem becomes:

$$
\begin{array}{cc}
\operatorname{maximize} & -\sum_{i=1}^{n} \lambda_{i}\left(\sigma_{i, n}^{\star}\left(T_{i}\right)\right)^{2} T_{i} \\
\text { s.t. } & -\left\|A-A_{h i s t}\right\|_{F r}-\sum_{i=1}^{n} \lambda_{i} \operatorname{Tr}\left(\Omega_{i} A\right) \succeq 0
\end{array}
$$

For a general overview of semi-definite programming algorithms see Vandenberghe and Boyd (1996), Nesterov and Nemirovskii (1994) or Alizadeh, Haeberly and Overton (1998). We followed the implementation structure given in Toh, Todd and Tütüncü (1996), having adapted in C the Mathematica algorithm by Brixius, Potra and Sheng (1996). Some more recent libraries including a more efficient formulation of the SOCP (quadratic, smoothness, euclidean distance ...) and L.P. constraints are available. These include the SEDUMI 1.1 library package by Sturm (1999) for symmetric cone programming, which we have extensively used here. One of the most efficient ways to use this library of function in Matlab is using CVX programming. CVX is a Matlab-based modeling system for convex optimization developed by Grant, Boyd and Ye (2005).

All modern solvers as SEDUMI 1.1 in Sturm (1999) or SDPT3 in Toh, Todd and Tütüncü (1998) 
can produce both primal and dual solutions to this problem. It is clear that this dual solution can be used for risk-management purposes, and it is shown here as a indicator of sensibility of our calibration problem.

\section{Numerical Results}

As a first attempt of calibration, let us use the well-known data from Brigo and Morini (2005). It will allow the reader to compare the results. Therefore, we have introduced the following inputs to the problem:

1. Initial curve of annual forward rates, as a vector with the following components (February 1st, 2002 from Brigo and Morini (2005))

$$
\begin{array}{ll}
F(0 ; 0,1) & 0.036712 \\
F(0 ; 1,2) & 0.04632 \\
F(0 ; 2,3) & 0.050171 \\
F(0 ; 3,4) & 0.05222 \\
F(0 ; 4,5) & 0.054595 \\
F(0 ; 5,6) & 0.056231 \\
F(0 ; 6,7) & 0.057006 \\
F(0 ; 7,8) & 0.057699 \\
F(0 ; 8,9) & 0.05691 \\
F(0 ; 9,10) & 0.057746
\end{array}
$$

2. Swaption Black volatilities (February 1st, 2002 from Brigo and Morini (2005)), where we substract just the following annual data, from 1 to 10 years,

$\begin{array}{ccccccccccc} & 1 y & 2 y & 3 y & 4 y & 5 y & 6 y & 7 y & 8 y & 9 y & 10 y \\ 1 y & 0.179 & 0.165 & 0.153 & 0.144 & 0.137 & 0.132 & 0.128 & 0.125 & 0.123 & 0.12 \\ 2 y & 0.154 & 0.142 & 0.136 & 0.13 & 0.126 & 0.122 & 0.12 & 0.117 & 0.115 & 0.113 \\ 3 y & 0.143 & 0.133 & 0.127 & 0.122 & 0.119 & 0.117 & 0.115 & 0.113 & 0.111 & 0.109 \\ 4 y & 0.136 & 0.127 & 0.121 & 0.117 & 0.114 & 0.113 & 0.111 & 0.109 & 0.108 & 0.107 \\ 5 y & 0.129 & 0.121 & 0.117 & 0.113 & 0.111 & 0.109 & 0.108 & 0.106 & 0.105 & 0.104 \\ 6 y & 0.125 & 0.118 & 0.114 & 0.1095 & 0.1075 & 0.106 & 0.105 & 0.104 & 0.1035 & 0.1025 \\ 7 y & 0.121 & 0.115 & 0.111 & 0.106 & 0.104 & 0.103 & 0.102 & 0.102 & 0.102 & 0.101 \\ 8 y & 0.118 & 0.112 & 0.1083 & 0.104 & 0.1023 & 0.1017 & 0.101 & 0.101 & 0.1007 & 0.1 \\ 9 y & 0.115 & 0.109 & 0.1057 & 0.102 & 0.1007 & 0.1003 & 0.1 & 0.1 & 0.0993 & 0.099 \\ 10 y & 0.112 & 0.106 & 0.103 & 0.1 & 0.099 & 0.099 & 0.099 & 0.099 & 0.098 & 0.098\end{array}$

3. Historical forward rate correlations (February 1, 2002 from Brigo and Morini (2005)),

$\begin{array}{ccccccccccc} & 1 y & 2 y & 3 y & 4 y & 5 y & 6 y & 7 y & 8 y & 9 y & 10 y \\ 1 y & 1 & 0.82 & 0.69 & 0.65 & 0.58 & 0.47 & 0.29 & 0.23 & 0.43 & 0.47 \\ 2 y & 0.82 & 1 & 0.8 & 0.73 & 0.68 & 0.55 & 0.45 & 0.4 & 0.53 & 0.57 \\ 3 y & 0.69 & 0.8 & 1 & 0.76 & 0.72 & 0.63 & 0.47 & 0.56 & 0.67 & 0.61 \\ 4 y & 0.65 & 0.73 & 0.76 & 1 & 0.78 & 0.67 & 0.58 & 0.56 & 0.68 & 0.7 \\ 5 y & 0.58 & 0.68 & 0.72 & 0.78 & 1 & 0.84 & 0.66 & 0.67 & 0.71 & 0.73 \\ 6 y & 0.47 & 0.55 & 0.63 & 0.67 & 0.84 & 1 & 0.77 & 0.68 & 0.73 & 0.69 \\ 7 y & 0.29 & 0.45 & 0.47 & 0.58 & 0.66 & 0.77 & 1 & 0.72 & 0.71 & 0.65 \\ 8 y & 0.23 & 0.4 & 0.56 & 0.56 & 0.67 & 0.68 & 0.72 & 1 & 0.73 & 0.66 \\ 9 y & 0.43 & 0.53 & 0.67 & 0.68 & 0.71 & 0.73 & 0.71 & 0.73 & 1 & 0.75 \\ 10 y & 0.47 & 0.57 & 0.61 & 0.7 & 0.73 & 0.69 & 0.65 & 0.66 & 0.75 & 1\end{array}$


The following figures compare some of the most relevant results, in terms of variance-covariance and correlation matrices:
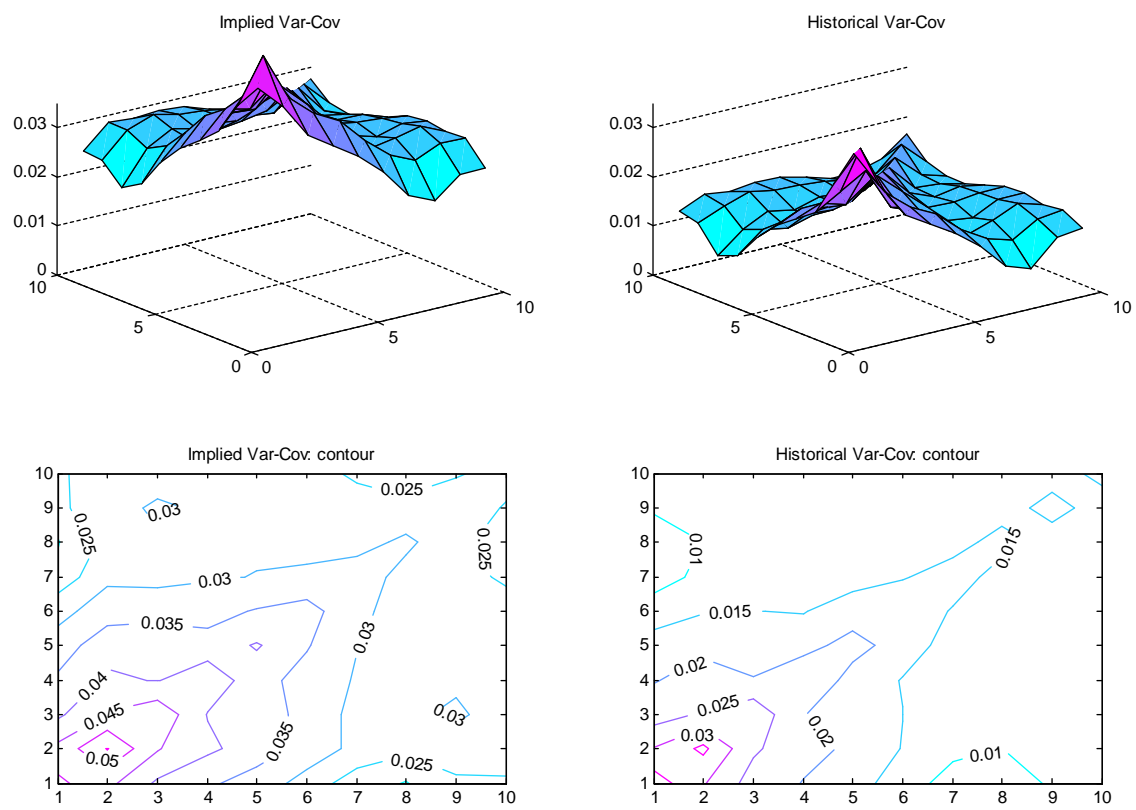

Figure (1) Covariance Matrix: Historical estimation vs. risk-neutral calibration

The solution of the primal SDP problem proposed here, is the following calibrated instantaneous forward volatility structure:

$\begin{array}{cccccccccc}0.2264 & 0.2369 & 0.2346 & 0.2351 & 0.2398 & 0.2445 & 0.2495 & 0.2625 & 0.2788 & 0.3099 \\ 0.2350 & 0.2394 & 0.2487 & 0.2565 & 0.2655 & 0.2734 & 0.2831 & 0.3011 & 0.3206 & 0 \\ 0.2208 & 0.233 & 0.2469 & 0.2563 & 0.2662 & 0.2766 & 0.2878 & 0.3055 & 0 & 0 \\ 0.2091 & 0.2251 & 0.2398 & 0.2492 & 0.2611 & 0.2724 & 0.2826 & 0 & 0 & 0 \\ 0.2011 & 0.218 & 0.2327 & 0.2441 & 0.2570 & 0.2672 & 0 & 0 & 0 & 0 \\ 0.1927 & 0.2091 & 0.2256 & 0.2379 & 0.2492 & 0 & 0 & 0 & 0 & 0 \\ 0.1834 & 0.2014 & 0.2183 & 0.2288 & 0 & 0 & 0 & 0 & 0 & 0 \\ 0.1770 & 0.1954 & 0.2100 & 0 & 0 & 0 & 0 & 0 & 0 & 0 \\ 0.1717 & 0.1871 & 0 & 0 & 0 & 0 & 0 & 0 & 0 & 0 \\ 0.1663 & 0 & 0 & 0 & 0 & 0 & 0 & 0 & 0 & 0\end{array}$



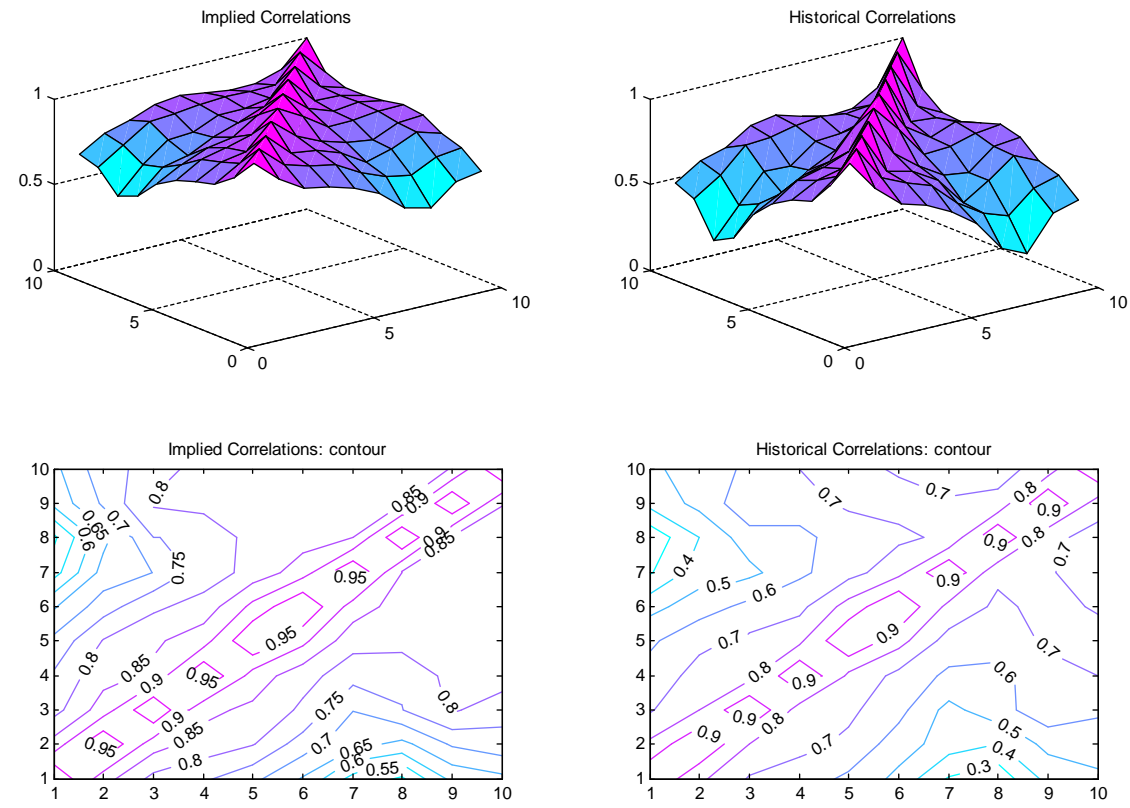

Figure (2) Correlation Matrices: Historical estimation vs. Risk-neutral calibration
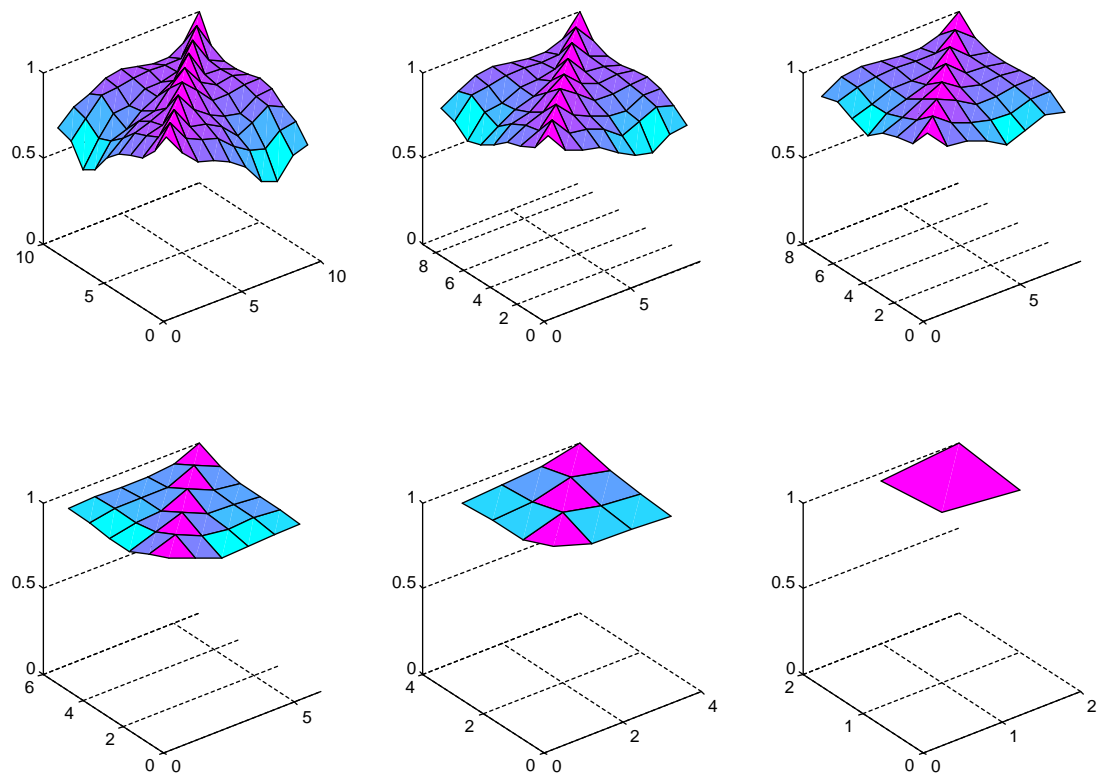

Figure (3) Implied Term Structure in the Correlation Matrix (first row:1y-2y-3y and second row: $4 \mathrm{y}-6 \mathrm{y}-8 \mathrm{y})$

Figures (1) and (2) show two interesting properties: First, how the shape of the implied covariance matrix and historical covariance matrix look quite similar. That is an expected consequence of the 
objective function. Second, the implied covariance matrix has higher value in absolute terms than the historical covariance matrix. This characteristic is usual in many different derivatives markets. Additionally, Figure (3) shows us the future dynamics of the variance-covariance matrix where the number of factors decrease with time. It is easy to see the effect of interpolations in the long term part of the volatility structure, basically because of the low liquidity of the 6,8 and 9 years swaptions.

The reader can compare that the numerical results from the original Brigo and Morini (2005) (after rank reduction to enforce positive forward volatilities). Let us briefly summarize here some differences:

- It is clear that a first relevant improvement is that the procedure here exposed guarantee a semidefinite positive covariance matrix, something essential to achieve a correct simulation in the full-rank LIBOR additive model.

- The second relevant property is that our procedure always guarantees a unique global solution. In some of the other cited methodologies, usually one has multiplicity of possible solutions, some of them with negative volatilities.

- And finally, this procedure does not require any rank reduction, and allows us the retrieval of a maximum amount of covariance information from the market.

\section{Computing sensitivity and risk-model management}

In this subsection, following d'Aspremont (2005), we investigate how the dual optimal solution can be exploited to manage the sensibility of the primal solution to movements in the market. Let us suppose that we have solved both the primal and the dual calibration problems above with market constraints $\sigma_{i, n}^{\star}\left(T_{i}\right)$ and let us denote with $X$ and $Y$ the optimal primal and dual solutions, respectively. Suppose also that the market price constraints in the original calibration problem are modified by a small amount $\Delta \in \mathbb{R}^{n}$. The new calibration problem becomes the following semidefinite program:

$$
\begin{array}{cc}
\text { minimize } & \left\|A-A_{\text {hist }}\right\|_{F r} \\
\text { subject to } & \operatorname{Tr}\left(\Omega_{i} A\right)=\left(\sigma_{i, n}^{\star}\left(T_{i}\right)\right)^{2} T_{i}+\Delta_{i} \quad \text { with } i=1, \ldots, n \\
& A \succeq 0
\end{array}
$$

where $A \in \mathbb{S}^{N}$ is the covariance matrix that we look for, and $A_{\text {hist }} \in \mathbb{S}^{N}$ is the historical covariance matrix, $k \in \mathbb{R}^{+}, \Omega_{i, n} \in \mathbb{S}^{N}$, and $\left(\sigma_{i, n}^{\star}\left(T_{i}\right)\right)^{2} T_{i} \in \mathbb{R}^{+}$is given by the swaption market as the Black (1976) cumulative variance of swaption on $S\left(0, T_{i}, T_{n}\right)$. If we note $A(\Delta)$ the primal optimal solution to the revised problem, we get the sensitivity of the solution to a change in market condition as:

$$
\frac{\partial A(\Delta)}{\partial \Delta_{i}}=-\lambda_{i}
$$

where $\lambda$ is the optimal solution to the dual problem (see Boyd and Vandenberghe (2004) for details). More specifically, the dual solution for the calibration problem proposed in 3.1.4 is (x 1.0e-007)

$\begin{array}{cccccccccc}0.15590 & 0.01058 & 0.06334 & 0.03071 & 0.17700 & 0.11090 & 0.55298 & 0.01178 & 0.07040 & 0 \\ 0.23461 & 0.01677 & 0.09225 & 0.06832 & 0.16764 & 0.12237 & 0.55440 & 0.04306 & 0.05752 & 0 \\ 0.26118 & 0.02001 & 0.16164 & 0.07118 & 0.21080 & 0.11018 & 0.24672 & 0.02131 & 0 & 0 \\ 0.25988 & 0.03141 & 0.16433 & 0.04552 & 0.24894 & 0.14307 & 0.37939 & 0 & 0 & 0 \\ 0.43288 & 0.03126 & 0.12174 & 0.04007 & 0.32598 & 0.11148 & 0 & 0 & 0 & 0 \\ 0.44196 & 0.02360 & 0.10298 & 0.05126 & 0.26754 & 0 & 0 & 0 & 0 & 0 \\ 0.35816 & 0.02253 & 0.14567 & 0.04492 & 0 & 0 & 0 & 0 & 0 & 0 \\ 0.32410 & 0.02378 & 0.11929 & 0 & 0 & 0 & 0 & 0 & 0 & 0 \\ 0.36771 & 0.02268 & 0 & 0 & 0 & 0 & 0 & 0 & 0 & 0 \\ 0.30537 & 0 & 0 & 0 & 0 & 0 & 0 & 0 & 0 & 0\end{array}$


This result, represented in Figure (4), shows the degree of stability of our primal solution, providing a direct indicator of robustness, but also it illustrates how a semidefinite programming based calibration allows to test various realistic scenarios at a minimum numerical cost and improves on the classical nonconvex methods that either had to "bump the market data and recalibrate" the model for every scenario with the risk of jumping from one local optimum to the next, or simulate unrealistic market movements by directly adjusting the covariance matrix.

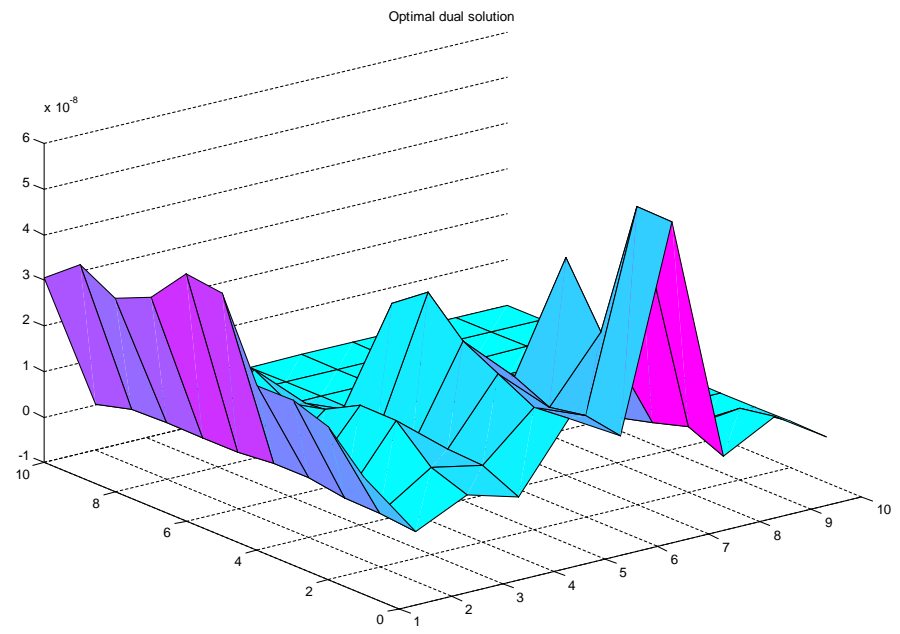

Figure (4) Sensibility of the primal solution (optimal dual solution)

\section{Robust dynamic calibration}

The previous sections were focused on how to compute the impact of a change in market conditions. Here we propose two different dynamic calibration solutions to dynamically provide a robust matrix for a certain period of market sessions (10 days). Let us assume that the initial problem (3.17) for $t=0$, therefore, in order to improve dynamically the robustness of our calibration, we solve the following sequence of problems

$$
\begin{array}{cc}
\text { minimize } & \left\|A^{(t)}-A^{(t-1)}\right\|_{F r} \\
\text { subject to } \operatorname{Tr}\left(\Omega_{i} A^{(t)}\right)=\left(\sigma_{i, n}^{\star(t)}\left(T_{i}\right)\right)^{2} T_{i} \quad \text { with } i=1, \ldots, n \\
A^{(t)} \succeq 0
\end{array}
$$

or, alternatively

$$
\begin{array}{ccc}
\operatorname{minimize} & \left\|A^{(t)}-A^{(t-1)}\right\|_{F r}+\varphi\left\|A^{(t)}-A_{h i s t}\right\|_{F r} \\
\text { subject to } & \operatorname{Tr}\left(\Omega_{i} A^{(t)}\right)=\left(\sigma_{i, n}^{\star(t)}\left(T_{i}\right)\right)^{2} T_{i} \quad \text { with } i=1, \ldots, n \\
A^{(t)} \succeq 0 &
\end{array}
$$

for every $t=1, \ldots, n$, where $A^{(t)} \in \mathbb{S}^{N}$ is the covariance matrix that we look for, and $A^{(t-1)} \in \mathbb{S}^{N}$ is the previous optimal covariance matrix solved in $t-1 ; i, n \in \mathbb{R}^{+}$and $1 \leq i \leq n, \Omega_{i, n} \in \mathbb{S}^{N}$, and $\left(\sigma_{i, n}^{\star(t)}\left(T_{i}\right)\right)^{2} T_{i} \in \mathbb{R}^{+}$is given by the swaption market as the Black (1976) cumulative variance of swaption with underlying $S\left(t, T_{i}, T_{n}\right)$, and $\varphi$, in problem (3.21), in a regularization constant.

Here, we have three examples of calibration of LIBOR market model proposed. In all cases, we have 
used the same forward term structure and swaptions volatilities, that the market quoted from March $12 t h$ to April, $200723 t h^{3}$. In the first example (Case 1), we solve the calibration related with the SDP problem (3.17), and the second example (Case 2) is related with the proposed robust dynamic calibration as the SDP problem (3.20) for the 10 consecutive mentioned market sessions. Additionally, we solve the third calibration problem, regularized for a $\varphi=0.1$, proposed as SDP problem in (3.21) as (Case 3).
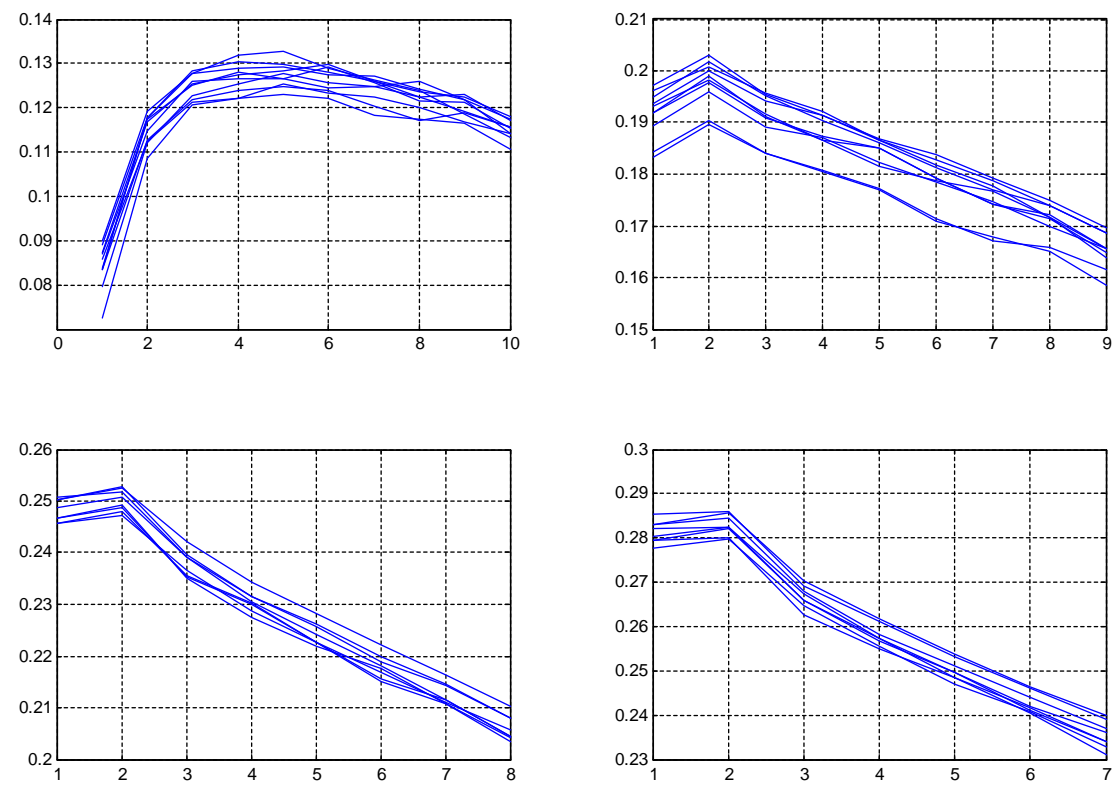

Figure(5) (Case 1) Dynamic solution from (3.17). Forward volatility structure calibrated using correlation (from initial period (10 factors) to fourth period ( 7 factors))

\footnotetext{
${ }^{3}$ Data courtesy of Caja Madrid, Fixed Income Derivatives desk.
} 

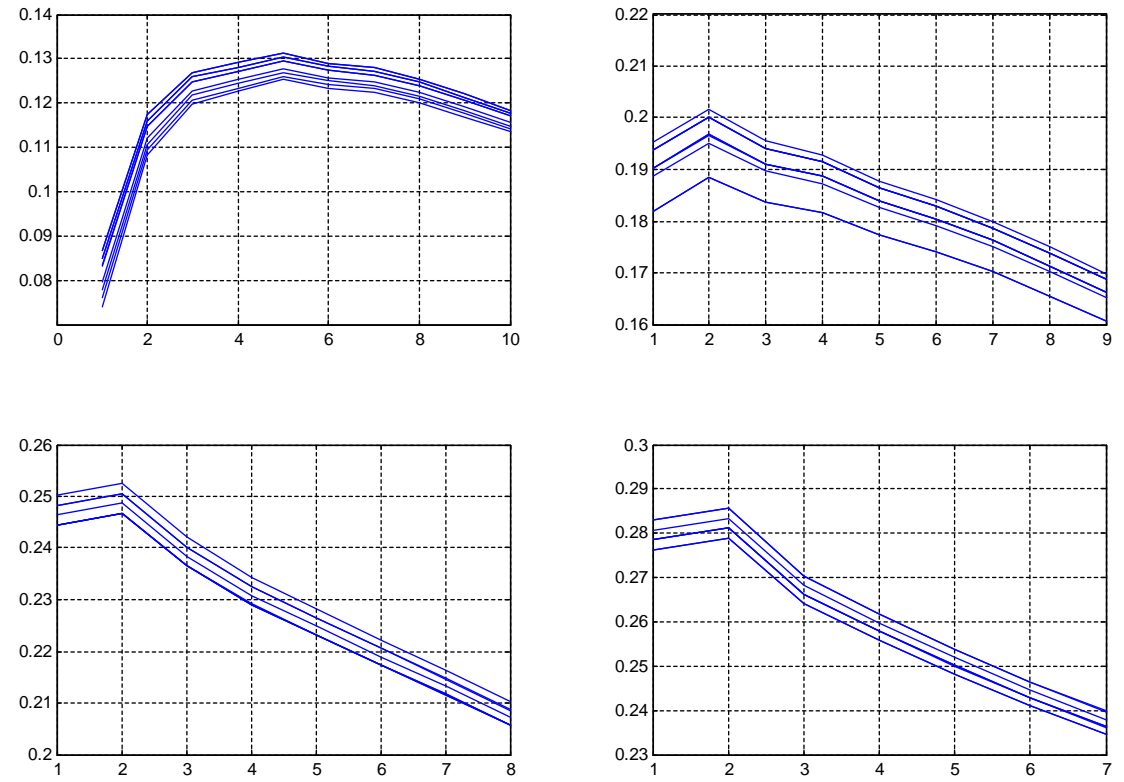

Figure(6) (Case 2) Dynamic solution from (3.20). Forward volatility structure calibrated using robust dynamic calibration (from initial period (10 factors) to fourth period ( 7 factors))
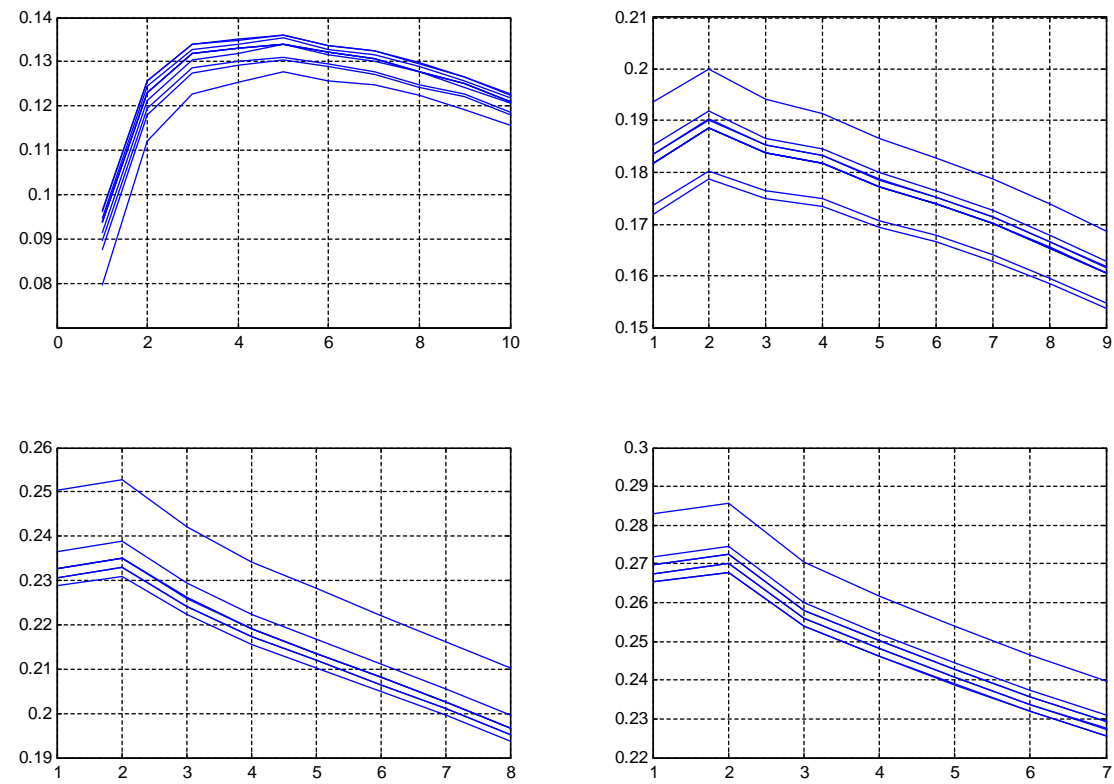

Figure (7) (Case 3 with $\varphi=0.1$ ) Dynamic solution from (3.21). Forward volatility structure calibrated using robust dynamic calibration (from initial period (10 factors) to fourth period ( 7 factors))

It is not surprising to see in Figure (5) and Figure (6) how the second case produces more stable 
or robust results than the first case. Because of that, the usual daily variation in the market prices will not affect the derivatives valuation and it will minimize the variability in the hedging and greeks computation. Figure (7) shows us an intermediate situation basically because we have introduced a regularization term in the objective function. This third case is useful if the trader wants to introduce any personal or historical view related with the covariance matrix but without loosing robustness.

\subsubsection{Second-step: the jump-part calibration}

In the previous section, we proposed a new method to obtain the calibration of the diffusion or continuous part, against the at-the-money swaption prices. However, during the whole thesis, we have introduced the jump framework in order to fit the implied forward volatility structure for in-the-money and out-ofthe-money swaptions implied volatility. In order to obtain a practical solution to the calibration problem and fit the smile, many authors have resorted to minimizing the in-sample quadratic pricing error (see, for example, Andersen and Andreasen (2000), Bates(1996)) or Cont and Tankov (2004) but always in the equity framework. Here, we extend some of these ideas to the Lévy-calibration problem in the swaptions market.

Basically the idea that we expose here is related with the calibration of a sequence of Lévy-measures under the jump-diffusion framework. As we have shown previously, the calibration of the diffusion part can be made just for the at-the-money swaption prices. It guarantees the compatibility with Black atthe-money prices. However, if the trader or practitioner wants to introduce the effect of the smile in the pricing of exotic derivatives, then we need to introduce a sequence of jump measures that adjust every of the maturities of the option, and for the different strikes.

For a predetermined collection of dates $0<T_{0}<T_{1}<\ldots<T_{n}$ with a fixed accrual period or tenor $\delta$, and for any $t \leq T_{i} \in\left[0, T^{\star}\right]$, let us denote by $L\left(t, T_{i}\right)$ the forward rate for the interval from $T_{i}$ to $T_{i+1}$, and $P S^{\sigma}\left(t, T_{\alpha}, T_{\beta},\left(x_{i, j}\right), v_{i}, K_{\alpha, \beta}\right)$ and $P S^{M}\left(t, T_{i}, T_{j}, \sigma_{i, j}^{\star}, K_{i, j}^{h}\right)$ are respectively the payer-swaption price given by our model and payer-swaption price given by the swaption market (according to Black (1976) model).

Following the similar idea in Andersen and Andreasen (2000) or Bates(1996) for equity markets, notice that we can formulate the following sequence of calibration problems, for every $i=1, \ldots, n$, we calibrate the Lévy measures minimizing the in-sample quadratic pricing error as

$$
\left(v_{i}\right)=\arg \inf \sum_{h=-m}^{m} \sum_{j=i+1}^{\beta}\left[\omega_{i j}^{h}\left|P S^{\sigma}\left(t, T_{i}, T_{j}, A_{i}, v_{i}, K_{i, j}^{h}\right)-P S^{M}\left(t, T_{i}, T_{j}, \sigma_{i, j}^{\star}, K_{i, j}^{h}\right)\right|^{2}\right]
$$

where $i, j \in\{1, \ldots, n\}$, with $i \leq j$ and $K_{i, j}^{h} \in\left\{K_{i, j}^{-m}, \ldots, K_{i, j}^{m}\right\}$ are the different strikes that the swaption market quotes, with $h \in \mathbb{N}$ such that if $h=0$ then $F S_{t}\left(K_{i, j}^{0}\right)=0$ (at-the-money case), or in other words if $K_{i, j}^{0}=F S_{i, j}$ then we have introduced at-the-money swaption, and no relevant information is added to the calibration problem because $P S^{\sigma}\left(t, T_{i}, T_{j}, A_{i}, v_{i}, F S_{i, j}\right)=P S^{M}\left(t, T_{i}, T_{j}, \sigma_{i, j}^{\star}, F S_{i, j}\right)$.

Let us recall that an European payer swaption is an option giving the right (and no obligation) to enter in a IRS at a given future time, the swaption maturity $T_{i}$. Usually the swaption maturity coincides with the first reset date of the underlying interest rates swap (IRS). The underlying IRS length $\left(T_{j}-T_{i}\right)$ is called the tenor of the swaption. As we have previously mentioned, it is the market practice to value swaptions with a Black (1976) formula.

Precisely let us define the price of a payer swaption at time $t$ as $P S^{M}\left(t, T_{i}, T_{j}, \sigma_{i, j}^{\star}, K_{i, j}^{h}\right)$ given by the swaption market, as a call-option to get into a swap that begins in $T_{i}$ and finish in $T_{j}$ with the swap rate $S\left(t, T_{i}, T_{j}\right)$ (underlying of the option) with strike $K_{i, j}$, and where $\sigma_{i, j}^{\star}$ is the Black (1976) cumulative variance of swaption on $S\left(t, T_{i}, T_{j}\right)$ for the mentioned strike $K_{i, j}$ quoted in the swaption market, such 
that

$$
P S^{M}\left(t, T_{i}, T_{j}, \sigma_{i, j}^{\star}, K_{i, j}\right)=\operatorname{Black}\left(t, T_{i}, T_{j}, \sigma_{i, j}^{\star}, K_{i, j}\right) \sum_{\beta=i+1}^{j} B\left(t, T_{\beta}\right) \delta_{\beta}
$$

On the other hand, the usual swaption definition that we have to price it using our model is

$$
\begin{aligned}
P S^{\sigma}\left(t, T_{i}, T_{j}, A_{i}, v_{i}, K_{i, j}^{h}\right) & =\mathbb{E}_{\mathbb{P}^{*}}\left\{B\left(t, T_{i}\right)\left(F S_{t}\left(K_{i, j}^{h}\right)\right)^{+} \sum_{\beta=i+1}^{j} B\left(t, T_{\beta}\right) \delta_{\beta}\right\} \\
& =\mathbb{E}_{\mathbb{P}^{*}}\left\{\sum_{\beta=i+1}^{j} B\left(t, T_{\beta}\right) \delta_{\beta}\left(\left(S\left(t, T_{i}, T_{j}\right)-K_{i, j}^{h}\right)\right)^{+}\right\} \\
& =\mathbb{E}_{\mathbb{P}^{*}}\left\{\left(\left(S\left(t, T_{i}, T_{j}\right)-K_{i, j}\right)\right)^{+}\right\} \sum_{\beta=i+1}^{j} B\left(t, T_{\beta}\right) \delta_{\beta} \\
& =\mathbb{E}_{\mathbb{P}^{*}}\left\{\left(\left(\sum_{\alpha=i}^{j-1} \omega_{\alpha}(t) L\left(t, T_{\alpha}\right)-K_{i, j}\right)\right)^{+}\right\} \sum_{\beta=i+1}^{j} B\left(t, T_{\beta}\right) \delta_{\beta}
\end{aligned}
$$

where

$$
\begin{aligned}
L\left(t, T_{i}\right)= & \sum_{j=1}^{\eta(t)}\left(L\left(t \wedge T_{j}, T_{i}\right)-L\left(T_{j-1}, T_{i}\right)\right) \\
= & L\left(0, T_{i}\right) \sum_{j=1}^{\eta(t)} \exp \left\{\left(\alpha_{j}\left(T_{j}, T_{i}\right)-\frac{1}{2} \sigma_{j}\left(T_{j}, T_{i}\right)^{2}\right)\left(t \wedge T_{j}-T_{j-1}\right)\right. \\
& \left.+\sigma_{j}\left(T_{j}, T_{i}\right)\left(W_{t \wedge T_{j}}-W_{T_{j-1}}\right)\right\} \prod_{l=N_{j-1}\left(T_{j-1}\right)}^{N_{j}\left(t \wedge T_{j}\right)} e^{Y_{l}}
\end{aligned}
$$

\section{An example of calibration under the double exponential jump-diffusion model}

Let us assume that our LIBOR additive process follows a jump-diffusion scheme or more specifically, a double exponential jump-diffusion or Kou (2003) model, which has two components, a continuous part modeled as Brownian motion, and a jump-part with jumps having a double exponential distribution and jump times driven by a Poisson process, assuming that

$$
G_{t}^{T^{\star}}:=\sum_{j \leq \eta(t)} \int_{T_{j}}^{t \wedge T_{j+1}} \alpha_{j}\left(s, T_{l}\right) d s+\sum_{j \leq \eta(t)} \int_{T_{j}}^{t \wedge T_{j+1}} \sigma_{j}\left(s, T_{l}\right) d W_{s}+\sum_{j \leq \eta(t)} \sum_{l=1}^{N_{j}(t)} Y_{l}
$$

where $\alpha_{j}$ under risk neutral measure has an specific form, $W_{t}$ is the standard Brownian motion, $N_{j}$ is a Poisson process with rate $\lambda_{j}$ and $Y_{i}$ is a sequence of independent and identically distributed of jumps with double exponential distribution i.e. the common density of $Y$ is given by

$$
f_{Y}(d y)=p \cdot \eta_{1} e^{-\eta_{1} y} 1_{\{y \geq 0\}}+q \cdot \eta_{2} e^{\eta_{2} y} 1_{\{y<0\}}
$$

where $p, q \geq 0, p+q=1, \lambda_{j} \geq 0$ for every $j=0,1, \ldots, n$, and $\eta_{1}>1, \eta_{2}>0$. Note that the means of the two exponential distribution are $1 / \eta_{1}$ and $1 / \eta_{2}$ respectively.

It is clear that the Fourier transform (or characteristic function) of $G_{t}^{T^{\star}}$ admits the (unique) repre- 
sentation given by the Lévy-Khintchine Theorem (Theorem 31 in Chapter 1), in this case

$$
\begin{aligned}
\hat{\mu}_{t}(z) & =\mathbb{E}\left[e^{\left\langle i z, G_{t}\right\rangle}\right] \\
& =\prod_{j \leq \eta(t)} \mathbb{E}\left(\exp \left[i z\left(G_{t \wedge T_{j+1}}-G_{T_{j}}\right)\right]\right) \\
& =\exp \left[\sum_{j \leq \eta(t)}\left(t \wedge T_{j+1}-T_{j}\right) \cdot \Psi_{j}(z)\right]
\end{aligned}
$$

where the characteristic exponent $\Psi_{j}(z)$ equals

$$
\Psi_{j}(z)=i\left\langle\alpha_{j}, z\right\rangle-\frac{1}{2}\left\langle z, A_{j} z\right\rangle+\int_{\mathbb{R}^{d}}\left(e^{i\langle z, y\rangle}-1-i\langle z, y\rangle 1_{\{|y| \leq 1\}}\right) v_{j}(d y) \quad \text { with } z \in \mathbb{R}^{d}
$$

where $A_{j}$ is a symmetric nonnegative-definite $(n-j+1) \times(n-j+1)$ matrix given as a solution by the first calibration problem, with $j=0,1, \ldots, n$, and $\alpha_{j}$ under risk-neutral measure follows (see Glasserman and Kou (2003) and Kou and Wang (2004))

$$
\begin{aligned}
\alpha_{j}\left(t, T_{i}\right)= & \sum_{k=\eta(t)}^{i} \frac{\delta \sigma_{j}\left(t, T_{k}\right) \sigma_{j}\left(t, T_{i}\right) L\left(t-, T_{k}\right)}{1+\delta L\left(t-, T_{k}\right)} \\
& +\int_{\mathbb{R}^{r}} y\left(1-\prod_{k=\eta(t)}^{i} \frac{1+\delta L\left(t-, T_{k}\right)}{1+\delta L\left(t-, T_{k}\right)(1+y)}\right) \lambda_{j}\left(p \cdot \eta_{1} e^{-\eta_{1} y} 1_{\{y \geq 0\}}+q \cdot \eta_{2} e^{\eta_{2} y} 1_{\{y<0\}}\right) d y
\end{aligned}
$$

and the Lévy measure can be defined as

$$
v_{j}(d y)=\lambda_{j}\left(p \cdot \eta_{1} e^{-\eta_{1} y} 1_{\{y \geq 0\}}+q \cdot \eta_{2} e^{\eta_{2} y} 1_{\{y<0\}}\right) d y
$$

where $p, q \geq 0, p+q=1, \eta_{2}>0$ and additionally the condition that $\eta_{1}>1$ is imposed to ensured that $L\left(t-, T_{i}\right)$ has finite expectations.

Therefore, the forward LIBOR rate can be described with the following SDE

$$
\frac{d L\left(t, T_{i}\right)}{L\left(t-, T_{i}\right)}=\alpha_{j}\left(t, T_{i}\right) d t+\sigma_{j}\left(t, T_{i}\right) d W_{t}+d\left(\sum_{l=1}^{N_{j}(t)} e^{Y_{l}}-1\right)
$$

Let us recall that we interpret the swap rate as a linear combination of forward rates, and in our case forward LIBOR rates, such that the payer swaption $P S^{\sigma}\left(t, T_{i}, T_{j}, A_{i}, v_{i}, K_{i, j}^{h}\right)$ may also be seen as

$$
\begin{aligned}
P S^{\sigma}\left(t, T_{i}, T_{j}, A_{i}, v_{i}, K_{i, j}^{h}\right) & =\mathbb{E}_{\mathbb{P} *}\left\{\sum_{\beta=i+1}^{j} B\left(t, T_{\beta}\right) \delta_{\beta}\left(S\left(t, T_{i}, T_{j}\right)-K_{i, j}^{h}\right)^{+}\right\} \\
& =\mathbb{E}_{\mathbb{P} \star}\left\{\left(\sum_{\beta=i+1}^{j} \omega_{\beta}(t) L\left(t, T_{\beta}\right)-K_{i, j}^{h}\right)^{+}\right\} \sum_{\beta=i+1}^{j} B\left(t, T_{\beta}\right) \delta_{\beta}
\end{aligned}
$$

Let us define the following two auxiliary variables

$$
\xi_{j}:=\ln \omega_{j}(0) L\left(0, T_{j}\right)
$$


and

$$
X_{t}:=\ln \sum_{j=1}^{\eta(t)} \exp \left\{\left(\alpha_{j}\left(s, T_{i}\right)+\frac{1}{2} \sigma_{j}\left(s, T_{i}\right)^{2}\right)\left(t \wedge T_{j}-T_{j}\right)+\sigma_{j}\left(s, T_{i}\right)\left(W_{t \wedge T_{j}}-W_{T_{j}}\right)\right\} \prod_{l=N_{j}\left(T_{j-1}\right)}^{N_{j}\left(t \wedge T_{j}\right)} e^{Y_{l}}
$$

then we have

$$
S\left(t, T_{i}, T_{j}\right)=\sum_{\beta=i}^{j-1} e^{\xi_{\beta}+X_{\beta}}
$$

will allows us to redefine the value of a payer swaption in $t$ as

$$
P S^{\sigma}\left(t, T_{\alpha}, T_{\beta}, \xi_{i}, K_{\alpha, \beta}\right)=\mathbb{E}_{\mathbb{P}^{*}}\left\{\left(\sum_{j=\alpha}^{\beta-1} e^{\xi_{j}+X_{j}}-K_{\alpha, \beta}\right)^{+}\right\} \sum_{j=\alpha+1}^{\beta} B\left(t, T_{j}\right) \delta_{j}
$$

We consider the modified payoff $\Phi\left(x, K_{\alpha, \beta}\right)=\left(\sum_{j=\alpha}^{\beta-1} e^{x_{j}}-K_{\alpha, \beta}\right)^{+}$and, for the sake of clarity, setting $\xi=\left(\xi_{j}\right)_{\alpha \leq j \leq \beta}, X=\left(X_{j}\right)_{\alpha \leq j \leq \beta}$ and $K_{\alpha, \beta}$ for $T_{\alpha}, T_{\beta}$ given inside the tenor structure $\left\{T_{1}, \ldots, T_{n}\right\} \in\left[0, T^{\star}\right]$,

$$
\begin{aligned}
P S^{\sigma}\left(\xi, K_{\alpha, \beta}\right) & =\mathbb{E}_{\mathbb{P} \star}\left\{\Phi\left(\xi+X, K_{\alpha, \beta}\right)\right\} \sum_{j=\alpha+1}^{\beta} B\left(t, T_{j}\right) \delta_{j} \\
& =\sum_{j=\alpha+1}^{\beta} B\left(t, T_{j}\right) \delta_{j} \cdot \int_{\mathbb{R}} \Phi\left(\xi+X, K_{\alpha, \beta}\right) \phi(x) d x
\end{aligned}
$$

where $\phi$ is the 'unknown' density of $X$ (however, we know the Fourier transform or Lévy-Khitchine characteristic function $\left.\hat{\mu}_{X}(z)=\prod_{\alpha+1 \leq j \leq \beta} \hat{\mu}_{X_{j}}(z)\right)$.

The expectation under $\mathbb{P}_{\alpha, \beta}$ can be computed inverting the Fourier transform, according with Raible (2000), in the following way

$$
\begin{aligned}
P S^{\sigma}\left(\xi, K_{\alpha, \beta}\right) & =\frac{1}{2 \pi i} \int_{R-i \infty}^{R+i \infty} e^{\xi z} \hat{\mu}_{X}(z) d z \\
& =\frac{1}{2 \pi} \int_{-\infty}^{+\infty} e^{\xi_{i}(R+i u)} \hat{\mu}_{X}(R+i u) d u \\
& =\frac{e^{\xi_{i} R}}{2 \pi} \lim _{N, M \rightarrow \infty} \int_{-M}^{N} e^{\xi_{i} i u} \hat{\mu}_{X}(R+i u) d u \\
& =\frac{e^{\xi_{i} R}}{2 \pi} \lim _{N, M \rightarrow \infty} \int_{-M}^{N} e^{\xi_{i} i u} \prod_{\alpha+1 \leq j \leq \beta} \hat{\mu}_{X_{j}}(R+i u) d u
\end{aligned}
$$

that can be solved numerically, after some additional transformations, using $\mathbf{F F T}^{4}$.

Fourier transforms have been widely used in valuing financial derivatives. For example, Carr and Madan (1998) propose Fourier transforms with respect to the log-strikes prices; Geman and Yor (1993), Fu, Madan and Wang (1999) use Fourier transform to price Asian options in the Black Scholes setting; Fourier transforms for the double-barrier and lookback options under the CEV model are given in Davydov and Linetsky (2001); Petrella and Kou (2004) use a recursion and Fourier transforms to price discretely monitored barrier and lookback options. Raible (2000) proposed a method for the evaluation of European stock options in a Lévy setting by using bilateral (or, two-sided) Fourier

${ }^{4}$ See Carr and Madan (1998) 
transforms. This approach is based on the observation that the pricing formula for European options can be represented as a convolution. Then one can use the fact that the bilateral Laplace transform of a convolution is the product of the bilateral Laplace transforms of the factors (the latter transforms are usually known explicitly).

Therefore, the second step calibration problem can be formulated in the following way

$$
\begin{gathered}
\left(\lambda_{i}, \eta_{1}, \eta_{2}, p\right)=\operatorname{arginf} \sum_{h=-m}^{m} \sum_{j=i+1}^{n}\left[\omega_{i j}^{h}\left\|P S^{\sigma}\left(t, T_{\alpha}, T_{\beta}, A_{i}, v_{i}, K_{\alpha, \beta}^{h}\right)-P S^{M}\left(t, T_{\alpha}, T_{\beta}, \sigma_{i, n}^{\star}, K_{\alpha, \beta}^{h}\right)\right\|\right] \\
p+q=1 \\
\text { s.t. } p, q \geq 0 \\
\eta_{1}>1, \eta_{2}>0
\end{gathered}
$$

where the weights $\omega_{i j}^{h}$ are positive and sum to one and they reflect the pricing error tolerance for the swaption with maturity $T_{\alpha}$ and swap ends at $T_{\beta}$ with strike $K_{\alpha, \beta}^{h}, P S^{M}\left(t, T_{\alpha}, T_{\beta}, \sigma_{i, n}^{\star}, K_{\alpha, \beta}^{h}\right)$ is directly given by the market price of a payer swaption, $K_{\alpha, \beta}^{h} \in\left\{K_{\alpha, \beta}^{-m}, \ldots, K_{\alpha, \beta}^{m}\right\}$ are the different strikes that the swaption market quotes, with $h \in \mathbb{N}$ such that if $h=0$ then $F S_{t}\left(K_{\alpha, \beta}^{0}\right)=0$ (at-the-money case).

\subsubsection{Numerical Performance}

The reliability of the two steps swaptions calibration depends mainly on the accuracy of the underlying approximation (3.14) and (3.15) in the first calibration step. This formula has already been tested, for instance by Brigo and Mercurio (2001) and Jackel and Rebonato (2000). Here we extend similar tests, based on Monte Carlo simulation of the LMM dynamics calibrated using semidefinite programming, and we compare, for payer and receivers european swaptions at-the-money, the estimated prices using the Monte Carlo versus the theoretical Black-76 swaption value.

As we have mentioned before, let us define a predetermined collection of dates $0<T_{0}<T_{1}<\ldots<T_{n}$ with a fixed accrual period or tenor $\delta$, and for any $t \leq T_{i} \in\left[0, T^{\star}\right]$, and by $L\left(t, T_{i}\right)$ we define the forward rate for the interval from $T_{i}$ to $T_{i+1}$, and $P S^{\sigma}\left(t, T_{\alpha}, T_{\beta},\left(x_{i, j}\right), v_{i}, K_{\alpha, \beta}\right)$ and $P S^{M}\left(t, T_{\alpha}, T_{\beta}, \sigma_{i, n}^{\star}, K_{\alpha, \beta}\right)$ are respectively the payer-swaption price given by our model and payer-swaption price given by the swaption market (according to Black (1976) model). Let us, first of all, discretize the continuous dynamics seen in Section 3.1.3. Taking logs in order to get the stronger convergence of Milstein scheme, one obtains for every $\alpha \leq j \leq \beta \leq n$ with

$$
\begin{aligned}
\log L\left(t+\Delta t, T_{j}\right)= & \log L\left(t, T_{j}\right)+\sigma_{j}(t) \sum_{i=\alpha+1}^{j} \frac{\rho_{i, j} \sigma_{i}(t) \delta_{i} L\left(t, T_{i}\right)}{1+\delta_{i} L\left(t, T_{i}\right)} \Delta t \\
& -\frac{\sigma_{j}(t)^{2}}{2} \Delta t+\sigma_{j}(t)(W(t+\Delta t)-W(t))
\end{aligned}
$$

where the instantaneous volatility $\sigma_{i}(t)$ and correlations $\rho_{i, j}(t)$ between the n-factors has been estimated solving the SDP problem (see Proposition 106) and they are piecewise stationary. The base scenario we use for most of our results sets $\delta=1$ year, $n=10$ years corresponding to a ten-years term structure of annual rates, and generating 10 points of data per year. Therefore $\alpha$ will take values between 1 and 5 , and $\beta$ between 1 and 10 (with $\alpha<\beta \leq 10$ ) where $T_{\alpha}$ indicates the expiry date of the option and $T_{\beta}$ is the maturity of the swaption. We generate 10.000 simulations under the terminal measure in order to reach a two-side $98 \%$ window, according to the standard error of Monte Carlo method ${ }^{5}$. All the swaptions priced here using this methodology, are at-the-money swaptions, and the data set used in this simulation are real market data (quoted March $12 t h, 2007)^{6}$.

\footnotetext{
${ }^{5}$ See Glasserman and Zhao (2000) for a complete description and implementation methodology.

${ }^{6}$ Market volatilities kindly provided by Caja Madrid Capital Markets (Fixed Income Derivatives Desk) using reliable
} 
Figure (8) compares the prices between both methodologies and estimated relative errors (in percentage) between the prices generated using Monte Carlo with a LMM and Black-76. In the x-axis, the swap maturity in years is represented as the market used to quoted swaptions, without the option term $(\beta-a)$, and in the z-axis, the option expiry (payer in the left and receiver in the right side) in years.
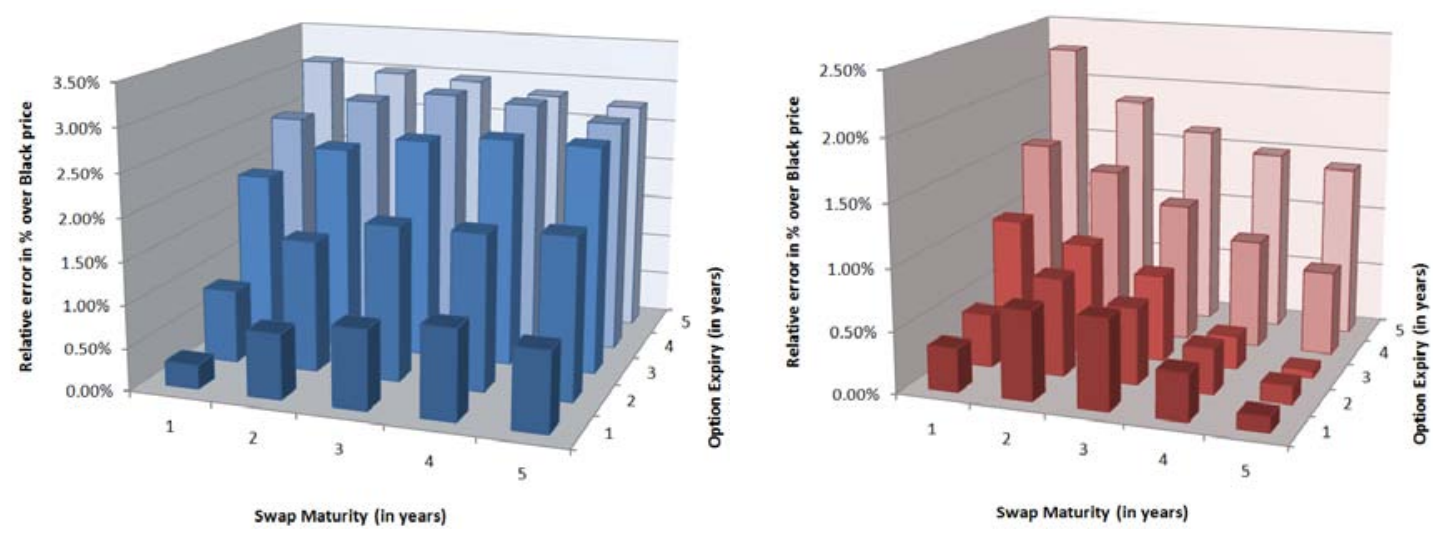

Figure (8): Relative error (in percentage) in the payer-swaption (left) and receiver-swaption (right) price using LMM-valuation (Monte Carlo) calibrated using SDP versus Black-76.

Not surprisingly, considering the structure of the SDP problem, Figure (8) shows that all swaptions seem to fit reasonably well, except for the longest underlying, in line with the d'Aspremont (2003) or Longstaff et al. (2000). With the exception of the payer-swaption with longest option expiry, in the rest of the cases, the price approximation appears good enough, and it confirms the accuracy of a full-rank calibration using SDP for at-the-money swaptions market (with 10 factors). It helps us to confirms the advantages of this methodology to price and hedge more sophisticated exotic swaptions, that usually have a great price dependence of the covariance structure.

market sources. All errors are my own. 


\section{Bibliography}

[1] Alizadeh, F., Haeberly, J. A. and Overton,M. L. (1998). Primal-dual interior-point methods for semidefinite programming: Convergence rates, stability and numerical results, SIAM Journal on Optimization 8, pp. 746-768.

[2] Alvarez O. and Tourin A. (1996). Viscosity solutions of nonlinear integro-differential equations, Ann. Inst. H. Poincaré Anal. Non Linéaire, 13 pp. 293-317.

[3] Andersen L. and Andreasen J. (2000). Jump-diffusion models: Volatility smile fitting and numerical methods for pricing, Rev. Derivatives Research, 4 pp. 231-262.

[4] Applebaum, D. (2004). Lévy Processes and Stochastic Calculus. Cambridge University Press.

[5] Ascher U. M. , Ruuth S. J., and Wetton B. T. R. (1995). Implicit-explicit methods for timedependent partial differential equations, SIAM J. Numer. Anal., 32 pp. 797-823.

[6] Avellaneda, M. (1998). Minimum entropy calibration of asset pricing models. International Journal of Theoretical and Applied Finance 1, pp. 447-72

[7] Avellaneda, M., Levy, A. and Paras, A. (1995). Pricing and hedging derivative securities in markets with uncertain volatilities, Applied Mathematical Finance 2, pp. 73-88.

[8] Avellaneda, M. and Paras, A. (1996). Managing the volatility risk of portfolios of derivative securities: the lagrangian uncertain volatility model, Applied Mathematical Finance 3, pp. 21-52.

[9] Bachelier, L. (1900). Théorie de la spéculation. Annales scientifiques de l'E.N.S. 3(17), pp. 21-86.

[10] Bakshi, G., Cao, C., and Chen, Z. (1997). Empirical Performance of Alternative Option Pricing Models, J. Finance 52, pp. 2003-2049.

[11] Barles G., Buckdahn R., and Pardoux, E., (1997). BSDEs and integral-partial differential equations, Stochastics Stochastics Rep., 60, pp. 57-83.

[12] Barles G. and Jakobsen E. R., (2002). On the convergence rate of approximation schemes for Hamilton-Jacobi-Bellman equations, M2AN Math. Model. Numer. Anal., 36, pp. 33-54.

[13] Barles G. and Souganidis P., (1991). Convergence of approximation schemes for fully nonlinear second order equations, Asymptotic Anal., 4, pp. 271-283.

[14] Barndorff-Nielsen, O. E. (1998). Processes of Normal Inverse Gaussian Type, Finance Stochast. 2, pp. 41-68.

[15] Barndorff-Nielsen, O.E., Pedersen, J., and Sato, K. (2001). Multivariate subordination, selfdecomposability and stability. Adv. Appl. Probab. 33, pp. 160-187.

[16] Barndorff-Nielsen, O. E., and Shepard, N. (2001). Non-Gaussian Ornstein-Uhlenbeck-Based Models and Some of Their Uses in Financial Economics, J.Roy. Statist. Soc.B, 63, pp. 167-241

[17] Barndorff-Nielsen, O.E., and Shephard, N. (2005). Continuous Time Approach to Financial Volatility. Cambridge University Press. (To appear) 
[18] Barndorff-Nielsen, O.E., and Shiryaev, A.N. (2005). Change of Time and Change of Measure. (In preparation.)

[19] Bates, D. S. (1996a). Jumps and stochastic volatility: the exchange rate processes implicit in Deutschmark options. Review of Financial Studies 9, pp. 69-107.

[20] Bates, D. S. (1996b). Testing option pricing models. In Statistical methods in finance, vol. 14 of Handbook of Statististics, pp. 567-611. North-Holland, Amsterdam.

[21] Bertoin, J. (1996). Lévy Processes. Cambridge University Press.

[22] Bielecki, T. and Rutkowski, M. (2002). Credit Risk: Modeling, Valuation and Hedging. Springer Verlag, Berlin Heidelberg New York

[23] Billingsley, P. (1968). Convergence of Probability Measures. John Wiley and Sons, New York.

[24] Billingsley, P. (1986). Probability and Measure. John Wiley and Sons, New York.

[25] Bingham, N.H., Goldie, C.M. and Teugels, J.L. (1987). Regular Variation, Cambridge University Press, Cambridge.

[26] Björk, T., G. Di Masi, Y. Kabanov and W. Runggaldier (1997). Towards a general theory of bond markets. Finance and Stochastics 1, pp. 141-174.

[27] Björk, T., Y. Kabanov, and W. Runggaldier (1997). Bond market structure in the presence of marked point processes. Mathematical Finance 7, pp. 211-239.

[28] Black, F. and Scholes, M. (1973). The pricing of options and corporate liabilities. Journal of Political Economy 81, pp. 637-654.

[29] Black, F. and Cox, J. (1976). Valuing corporate securities: some effects on bond indenture provisions. Journal of Finance 31, pp. 351-367.

[30] Boyd, S. and Vandenberghe, L. (2004). Convex Optimization, Cambridge University Press.

[31] Brace, A., Dun, T. and Barton, G. (1999). Towards a central interest rate model. Working Paper. FMMA

[32] Brace, A., D. Gatarek and M. Musiela (1997). The market model of interest rate dynamics. Mathematical Finance 7, pp. 127-155.

[33] Brace, A. and Womersley, R. S. (2000). Exact fit to the swaption volatility matrix using semidefinite programming,Working paper, ICBI Global Derivatives Conference .

[34] Breiman, L. (1968). Probability, Addison-Wesley, Reading, Mass., (republished SIAM, Philadelphia, 1992)

[35] Brémaud, P. (1980). Point processes and Queues. Martingale dynamics. Springer Series in Statistics. Springer Verlag, Berlin Heidelberg New York.

[36] Brigo, D. and Mercurio F. (2001). Interest Rate Models: Theory and Practice. Springer.

[37] Brigo D. Mercurio F. and Morini M., (2005). The Libor Model Dynamics: Approximations, Calibration and Diagnostics, European Journal of Operation Research 163, pp. 30-41.

[38] Brigo D. and Mercurio F. (2002). Calibrating LIBOR. Risk Magazine, January issue.

[39] Brigo D., Mercurio F. and Rapisarda, F. (2004). Smile at Uncertainty, Risk, May issue.

[40] Brigo D. and Morini M. (2006). Efficient Analytical Cascade Calibration of the LIBOR market model with Endogenous Interpolation. Accepted for publication in the Journal of Derivatives.

[41] Carr, P., Geman, H., Madan, D. and Yor, M. (2002). The fine structure of asset returns: An empirical investigation J.Bus. 75, pp. 305-332. 
[42] Carr, P., Geman, H., Madan, D. and Yor, M. (2005). Pricing options on realized variance. Finance Stochast. 9, pp. 453-475.

[43] Carr, P., Geman, H., Madan, D. and Yor, M. (2003). Stochastic Volatility for Lévy Processes. Mathematical Finance 13, pp. 345-382.

[44] Carr, P. and Madan, D. (1998). Option Valuation using the Fast Fourier Transform. Journal of Computational Finance 2, pp. 61-73.

[45] Chan, T. (1999). Pricing contingent claims on stocks driven by Lévy processes. Annals of Applied Probability, 9(2), pp. 504-528.

[46] Chambers, J. M., Mallows, C.L., and Stuck, B.W. (1976) A method for simulating stable random variables. J. amer. Statist. Assoc. 71 pp. 340-344.

[47] Cont, R. (2001). Inverse problems in financial modeling: theoretical and numerical aspects of model calibration., Lecture Notes, Princeton University.

[48] Cont, R. and Tankov P. (2004). Financial modelling with Jump Processes. CRC Press.

[49] Cont, R. and Tankov P. (2004). Nonparametric calibration of jump-diffusion option pricing models, J. Comput. Finance, 7, pp. 1-49.

[50] Cont, R. and Voltchkova E. (2005). Integro-differential equations for option prices in exponential Lévy models, Finance Stoch., 9 pp. 299-325.

[51] Crandall M. G., Ishii H., and Lions P.-L. (1992). User's guide to viscosity solutions of second order partial differential equations, Bull. Amer. Math. Soc. (N.S.), 27 , pp. 1-67.

[52] Crandall M. G. and Lions P.-L. (1984). Two approximations of solutions of Hamilton-Jacobi equations, Math. Comp., 43, pp. 1-19.

[53] d'Aspremont, A. (2002) Interest Rate Model Calibration and Risk-Management using Semidefinite Programming, PhD thesis, Ecole Polytechnique.

[54] d'Aspremont, A. (2003) Interest Rate Model Calibration using Semidefinite Programming. Applied Mathematical Finance 10, pp. 183-213.

[55] d'Aspremont, A. (2005) Risk-Management Methods for the Libor Market Model Using Semidefinite Programming. J Computational Finance 8, pp. 77-99.

[56] Davydov, D. and Linetsky, V. (2002) Structuring, Pricing and Hedging Double Barrier Step Options, J. Comput. Finance, 5-2, pp. 55-87.

[57] Doob, J. L., (1953) Stochastic Processes, Wiley.

[58] Doob, J. L., (1954) Semimartingales and subharmonic functions, Trans. Amer. Math. Soc. 77, pp. $86-121$.

[59] Duffee, Gregory R., (1998). The relation between treasury yields and corporate bond yield spreads, Journal of Finance 53, pp. 2225-2241.

[60] Duffee, Gregory R., (1999). Estimating the price of default risk, The Review of Financial Studies 12, pp. 187-226.

[61] Duffie, D. and Ming Huang, (1996). Swap rates and credit quality, Journal of Finance 51, pp. 921-949.

[62] Duffie, D. and Rui Kan, (1996). A yield-factor model of interest rates, Mathematical Finance 6, pp. 379-406.

[63] Duffie, D. and Singleton, K. (1999). Modeling term structures of defaultable bonds, Review of Financial Studies 12, pp. 687-720. 
[64] Duffie, D. and Singleton, K. (1997). An econometric model of the term structure of interest-rate swap yields, Journal of Finance 52, pp. 1287-1381.

[65] Duffie, D. and Lando D. (2001). Term structure of credit spreads with incomplete accounting information, Econometrica, 69, pp. 633-664.

[66] Duffie, D. and Protter, P. (1992). From discrete to continuous-time finance: weak convergence of the financial gain processes. Mathematical Finance, 2(1), pp. 1-15.

[67] Dzhaparidze, K. and Valkeila, E. (1990). On the Hellinger type distances for filtered experiments. Probability Theory and related Fields 85, pp. 105-117.

[68] Eberlein, E. (2001). Application of generalized hyperbolic Lévy motions to finance. In O. E. Barndorff-Nielsen, T. Mikosch, and S. Resnick (Eds.), Lévy Processes: Theory and Applications, pp. 319-337. Birkhäuser Verlag.

[69] Eberlein, E., Jacod, J. and Raible, S. (2005). Lévy term structure models: no-arbitrage and completeness. Finance and Stochastics 9, pp. 67-88.

[70] Eberlein, E. and Kluge, W. (2006). Exact pricing formulae for caps and swaptions in a Lévy term structure model. Journal of Computational Finance 9 (2), pp. 99-125

[71] Eberlein, E. and Kluge, W. (2006). Valuation of floating range notes in Lévy term structure models. Mathematical Finance 16, pp. 237-254.

[72] Eberlein, E., Kluge, W. and Papapantoleon, A. (2006). Symmetries in Lévy term structure models. International Journal of Theoretical and Applied Finance 9 (6), pp. 967-986.

[73] Eberlein, E., Keller, U. and Prause, K. (1998). New Insights into Smile, Mispricing and Value at Risk, J. Business 71, pp. 371-406.

[74] Eberlein, E. and Özkan, F. (2005). The defaultable Lévy term structure: Ratings and restructuring. Preprint Nr. 71, Freiburg Center for Data Analysis and Modeling, University of Freiburg.

[75] Eberlein, E. and Raible, S. (1999). Term structure models driven by general Lévy processes. Mathematical Finance 9, pp. 31-53.

[76] El Karoui, N., Jeanblanc-Picqué, M. and Shreve, S. E. (1998). On the robustness of the BlackScholes equation, Mathematical Finance 8, pp. 93-126.

[77] El Karoui, N. and Quenez, M. (1995). Dynamic programming and pricing of contingent claims in an incomplete market, Siam Journal of Control and Optimization 33, pp. 29-66.

[78] Engl, H. W., Hanke, M. and Neubauer, A. (1996). Regularization of inverse problems. Vol. 375 of Mathematics and its Applications, Kluwer Academic Publishers Group, Dordrecht.

[79] Embrechts, P., and Maejima, M. (2002). Self-Similar Processes. Princeton Series in Applied Mathematics. Princeton, NJ, Princeton University Press.

[80] Esche, F., and Schweizer, M. (2003). Minimal entropy preserves the Lévy property: How and why, Preprint, Technical University Berlin.

[81] Ethier, S. N. and Kurtz, T. G. (1986). Markov Processes. Characterization and Convergence. John Wiley and Sons, New York.

[82] Fitzsimmons, P. (1987). A Converse to a Theorem of P. Lévy, Ann. Probab. 15, pp. 1515-1523.

[83] Fu, M. C., Madan, D. and Wang, T. (1999). Pricing Continuous Time Asian Options. Comparison of Monte Carlo and Laplace Transform Inversion Methods. J. of Computational Finance, 2, 2, pp. 49-74

[84] Gihman, I. I. and Skorohod, A. V. (1982). Stochastic differential equations and their applications. Kiev. Naukova dumka. 
[85] Glasserman, P. and Kou, S. (2001). The term structure of simple forward rates with jump risk. Preprint, Columbia University.

[86] Goll, T., and Kallsen, J. (2000). Optimal portfolios for logarithmic utility Stochastic Processes and their Applications, 89(1), pp. 31-48.

[87] Grigelionis, B. (1978). Additive Markov Processes, Lithuanian Math. J. Vol.18.3, pp. 340-342.

[88] Grigelionis, B. (1999). Processes of Meixner Type, Lithuanian Math. J. 39, pp. 33-41.

[89] Grigelionis, B. and Mikulevicius, R. (1981). Weak Convergence of semimartingales, Lithuanian Math. J. Vol.21.3, pp. 213-224.

[90] Grigelionis, B. and Mikulevicius, R. (1981). Weak Convergence of sthochastic point processes, Lithuanian Math. J. Vol.21.4, pp. 297-301.

[91] Grigelionis, B. and Mikulevicius R. (1983). On stably weak convergence of semimartingales and of point processes, Theory Proab. Appl., Vol.28.2, pp. 337-350.

[92] Halmos, P. R. (1950) Measure theory, Van Nostrand Pricenton, NJ.

[93] Harrison, J.M. and Kreps, D. M. (1979). Martingales and Arbitrage in Multiperiod Security Markets. Journal of Economic Theory, 20, pp. 381-408.

[94] Harrison, J. M. and Pliska S. (1981). Martingales and Stochastic Integrals in hte Theory of Continuous Trading. Stochastic processes and Their Applications, 11, pp. 215-260.

[95] He, S. W., Wang, J. G. and Yan, J. A. (1992). Semimartingale Theory and Stochastic Calculus. Science Press, Beijing.

[96] Heath, D., Jarrow, R. and Morton, A. (1992). Bond pricing and the term structure of interest rates: A new methodology for contingent claims valuation. Econometrica 60, pp. 77-105.

[97] Hoover, D. N. (1991) Convergence in distribution and Skorokhod convergence for the general theory of processes. Probability Theory and Related Fields, 89(3), pp. 239-259.

[98] Jacod, J. (1975). Multivariate point processes: predictable projection, Radon-Nikodym derivative, representation of martingales. Z.Wahrsch. Verw. Geb. 31 pp. 235-253.

[99] Jacod, J. (1979). Calcul stochastique et problémes de martingales. Lecture Notes in Mathematichs 714. Springer.

[100] Jacod, J. (1985). Théorèmes limite pour les processus. Ecole d'été de St-Flour XIII 1983. Lecture Notes in Mathematics 1117, Springer.

[101] Jacod, J. and Shiryaev, A. N. (1987). Limit Theorems for Stochastic Processes. Springer.

[102] Jakubowski, A., Mémin, J. and Pagès, G. (1989) Convergence en loi des suies d'integrales stochastiques sur l'espace $D^{1}$ de Skorokhod. Probability Theory and Related Fields, 81(1), pp. 111-137.

[103] Jamshidian, F. (1997). LIBOR and swap market models and measures. Finance and Stochastics 1, pp. 293-330.

[104] Jamshidian, F. (1999). Libor market model with semimartingales. Working paper, NetAnalytic Ltd.

[105] Jarrow, R., Lando, D., and Turnbull, S. (1997). A Markov Model tor the Term Structure of Credit Spreads. Review of Financial Studies 10, 48 pp. 1-523.

[106] Jarrow, R., and Turnbull, S. (1995). Pricing Options on Financial Securities Subject to Default Risk. Journal of Finance 5-0, pp. 53-86.

[107] Jeanblanc, M., Pitman, J. and Yor, M. (2001). Self-Similar Processes with Independent Increments Associated with Lévy and Bessel Processes, Stochast. Proc. Appl. 100, pp. 223-232. 
[108] Kallenberg, O. (1997). Fundations of Modern Probability. Springer Verlag, Berlin.

[109] Kallsen, J. and Shiryaev, A. N. (2001). Time change representation of stochastic integrals. Theory of Probability and Its Applications. Russian Edition; English Version: http://www.stochastik.unifreiburg.de/homepages/kallsen/.

[110] Kallsen, J. and Shiryaev, A. N. (2002). The cumulant process and Esscher's change of measure. Finance and Stochastics 6, pp. 397-428.

[111] Karatzas, I. and Shreve, S. E. (1987). Limit theorems for stochastic processes. Springer Verlag, Berlin.

[112] Karmarkar, N. K. (1984). A new polynomial-time algorithm for linear programming, Combinatorica 4, pp. 373-395.

[113] Khintchine, A. Y. (1938). Limit Laws of Sums of Independent Random Variables, ONTI, Moscow, Russia.

[114] Knight, F. B. (2001). On the Path of an Inert Object Impinged on One Side by a Brownian Particle, Probab. Theory Related Fields 121, pp. 577-598.

[115] Kolmogorov, A.N. (1950). Foundations of the Theory of Probability, Chelsea Pub. New York (German original 1933)

[116] Konikov, M., and D. Madan (2002). Option Pricing using Variance Gamma Markov Chains, Rev. Derivatives Res. 5, pp. 81-115.

[117] Kou, S. G. (2002). A jump diffusion model for option pricing. Management Science. Vol. 48, pp. 1086-1101.

[118] Kou, S. G. Petrella G. and Wang H. (2005). Pricing path-dependent options with jump risk via Laplace transforms. Kyoto Economic Review. Vol. 74, pp. 1-23.

[119] Kou, S. G. and Wang, H (2004). Option pricing under a double exponential jump diffusion model. Management Science. Vol. 50, pp. 1178-1192.

[120] Kurtz, T. G. and Protter, P. E. (1991) Weak limit theorems for stochastic integrals and stochastic differential equations. Annals of Probability, 19(3), pp. 1035-1070.

[121] Kurtz, T. G. and Protter, P. E. (1996) Weak convergece of stochastic integrals and differential equations. Lecture notes in Mathematics 1627, 1-41 Edit. Springer Verlag, Berlin.

[122] Lamperti, J. (1962). Semi-stable Stochastic Processes, Trans. Am. Math. Soc. 104, pp. 62-78.

[123] Lévy, P. (1937) Théorie de l'Addition des Variables Aléatoires. Paris, Gauthier-Villars.

[124] Lindvall, T. (1973). Weak convergence of probability measures and random functions in the function space $\mathrm{D}([0, \infty[)$ J. Appl. Probab., Vol. 10, pp. 109-121.

[125] Liptser, R. Sh. and Shiryaev, A. N. (1977). Statistics of Random processes, I: General theory. Springer Verlag.

[126] Liptser, R. Sh. and Shiryaev, A. N. (1989). Theory of martingales. Mathematics and its applications, Kluwer Academic Publishers.

[127] Lobo,M., Vandenberghe, L., Boyd, S. and Lebret, H. (1998). Applications of second-order cone programming, Linear Algebra and its Applications 284, pp. 193-228. Special Issue on Linear Algebra in Control, Signals and Image Processing.

[128] Loève, M. (1977). Probability theory, vol. 1 and 2, 4th ed. New York, Springer

[129] Longstaff, F. A., Santa-Clara, P. and Schwartz, E. S. (2000). The relative valuation of caps and swaptions: Theory and empirical evidence., Working Paper, The Anderson School at UCLA. 
[130] Madan, D. (2001). Financial modeling with discontinuous price processes. In Barndorff-Nielsen,O. E., Mikosch, T. and Resnick, S.(eds), Lévy processes - theory and applications. Birkhauser, Boston.

[131] Madan, D.B., Carr, P. and Chang, E.C. (1998). The variance gamma process and option pricing. European Finance Review 2, pp. 79-105.

[132] Madan, D.B. and Milne, F. (1991). Option pricing with V.G. martingale components. Mathematical Finance 1(4), pp. 39-55.

[133] Maejima, M., and Sato, K. (2003). Semi-Lévy processes, semi-selfsimilar additive processes, and semi-stationary Ornstein-Uhlenbeck type processes. J. Math. Kyoto Univ. 43, pp. 609-639.

[134] Maejima, M., Sato, K., and Watanabe, T. (2000). Distributions of selfsimilar and semiselfsimilar processes with independent increments. Statist. Probab. Letters 47, pp. 395-401.

[135] Maejima, M., Suzuki, K., and Tamura, Y. (1999). Some multivariate infinitely divisible distributions and their projections. Probab. Theory Math. Statist. 19, pp. 421-428.

[136] Maruyama, G. (1970). Infinitely divisible processes. Theory Probab. Appl. 15, pp. 1-22.

[137] Mandelbrot, B. B. (1963). New methods in statistical economics, Journal of Political Economy, 71, pp. 421-440.

[138] Marsaglia, G. and Tsang, W.W. (2000). A Simple Method for Generating Gamma Variables, ACM Trans. Math. Soft. 26(3) pp. 363-372.

[139] Maruyama, G. (1970). Infinitely divisible processes. Theory Probab. Appl. 15, pp. 1-22.

[140] Merton, R. (1976). Option pricing when underlying stock returns are discontinuous. Journal of Financial Economics 3, pp. 125-44.

[141] Merton, R. (1974). On the pricing of corporate debt: the risk structure of interest rates. Journal of Finance 29, pp. 449-470.

[142] Miltersen, K. R., K. Sandmann, and D. Sondermann (1997). Closed form solutions for term structure derivatives with log-normal interest rates. Journal of Finance 52, pp. 409-430.

[143] Miyahara, Y., and Fujiwara, T. (2003). The minimal entropy martingale measures for geometric Lévy processes. Finance and Stochastics 7, pp. 509-31.

[144] Morozov, V. (1966). On the solution of functional equations by the method of regularization. Soviet Mathematics Doklady 7, pp. 414-17.

[145] Musiela, M. and Rutkowski, M. (1997). Continuous-time term structure models: forward measure approach. Finance and Stochastics 1, pp. 261-291.

[146] Musiela, M. and Rutkowski, M. (2004). Martingale Methods in Financial Modelling (2nd ed.). Springer.

[147] Nemirovskii, A. S. and Yudin, D. B. (1979). Problem complexity and method efficiency in optimization, Nauka (published in English by John Wiley, Chichester, 1983).

[148] Nesterov, Y. and Nemirovskii, A. (1994). Interior-point polynomial algorithms in convex programming, Society for Industrial and Applied Mathematics, Philadelphia.

[149] Nualart, D. and Schoutens, W. (2000). Chaotic and predictable representations for Lévy processes. Stochastic Processes and their Applications 90(1), pp. 109-122.

[150] Nualart, D. and Schoutens W. (2001). Backwards Stochastic Diferential Equations and FeynmanKac Formula for Lévy Processes, with applications in finance. Bernoulli, 7(5), pp. 761-776.

[151] Pardoux, E., Pradeilles, F. and Rao, Z. (1997). Probabilistic interpretation fo a system of semi-linear parabolic partial differential equations. Ann. Inst. Henri Poincaré. Vol. 33, n. 4, pp. 467-490 
[152] Pedersen, J., and Sato, K.-I. (2003). Cone-parameter convolution semigroups and their subordination. Tokyo J. Math. 26, pp. 503-525

[153] Pelsser, A. (2000). Efficient Methods for Valuing Interest Rate Derivatives. Springer Verlag

[154] Petrella, G. and Kou, S. G. (2004) Numerical pricing of discrete barrier and lookback options via Laplace transforms. J. of Computational Finance, 8. pp. 1-37

[155] Pfaff, T. (1982). Quick consistency of quasi maximum likelihood estimators. Annals of Statistics, Vol. 10, n3, pp. 990-1005

[156] Prause, K. (1997). Modelling financial data using generalized hyperbolic distributions. FDM Preprint 48, University of Freiburg.

[157] Press,W. H., Teukolsky, S. A., Vetterling,W. T., and Flannery, B. P. (1992). Numerical Recipes in C: the art of scientific computing. Cambridge University Press.

[158] Prigent, J.-L. (1999). Incomplete markets: Convergence of option values under the minimal martingale measure. Advances in Applied Probability, 31(4), pp. 1058-1077.

[159] Protter, P. (1995). Stochastic Integration and Differential Equations (2. ed.). Springer.

[160] Raible, S. (2000). Lévy processes in finance: theory, numerics, and empirical facts. Ph. D. thesis, University of Freiburg.

[161] Rebonato, R. (1998). Interest-Rate Options Models, Financial Engineering,Wiley.

[162] Rebonato, R. (1999). On the simultaneous calibration of multi-factor log-normal interest rate models to black volatilities and to the correlation matrix., QUARCH Working paper.

[163] Rocha-Arteaga, A., and Sato, K.-I. (2003). Topics in Infinitely Divisible Distributions and Lévy Processes. Aportaciones Matematicas, Investigacion 17, Sociedad Matematica Mexicana.

[164] Samperi, D. (2002). Calibrating a diffusion pricing model with uncertain volatility: regularization and stability. Mathematical Finance 12, pp. 71-87.

[165] Sato, K.-I. (1991). Self-similar processes with independent increments. Probab. Th. Rel. Fields 89, pp. 285-300.

[166] Sato, K.-I. (1998). Multivariate distributions with selfdecomposable projections. J. Korean Math. Soc. 35 , pp. $783-791$.

[167] Sato, K.-I. (1999). Lévy Processes and Infinitely Divisible Distributions. Cambridge University Press.

[168] Sato, K.-I. (2000). Density transformation in Lévy processes. MaPhySto Lecture Notes No. 7 , University of Aarhus.

[169] Sato, K.-I. (2004). Stochastic integrals in additive processes and application to semi-Lévy processes. Osaka J. Math. 41, pp. 211-236.

[170] Slominski, L. (1989). Stability of strong solutions of stochastic differential equations. Stochastic processes and their Applications, 31(2), pp. 173-202.

[171] Schmidt, T. and Stute, W. (2004). Credit risk - a survey. Contemporary Mathematics 336, pp. $75-115$.

[172] Schönbucher, Philipp J. (1998). Term structure modelling of defaultable bonds. The Review of Derivatives Studies, Special Issue: Credit Risk, 2, pp. 161-192.

[173] Schönbucher, Philipp J. (2002). A Tree implementation of a Credit Spread Model for Credit Derivatives. J. of Computational Finance, Vol. 6, No. 2, pp. 1-38. 
[174] Schönbucher, Philipp J. (2003). Credit Derivatives Pricing Models. Wiley.

[175] Schönbucher, Philipp J. (2003). Information-driven default contagion. Working paper, D-Math, ETH Zurich, December.

[176] Schönbucher, Philipp J. and Schubert, D. (2001). Copula-dependent default risk in intensity models. Working paper, Department of Statistics, Bonn University.

[177] Schoutens, W. (2003). Lévy Processes in Finance. Pricing Financial Derivatives. Wiley.

[178] Schoutens, W. (2000). The Meixner Process in Finance. Eurandom Report 2001-002, Eurandom, Eindhoven.

[179] Sibeux, C. and Vostrikova, L. (1996). Uniform convergence of semimartingales and minimum distance estimators. LAREMA Prepublication n. 29.

[180] Skorohod, A.V. (1991). Random Processes with Independent Increments. Kluwer Academic Pub., Dordrecht, Netherlands.

[181] Sturm, J. F. (1999). Using sedumi 1.0x, a matlab toolbox for optimization over symmetric cones, Optimization Methods and Software 11, pp. 625-653.

[182] Todd, M. and Yildirim, E. A. (2001). Sensitivity analysis in linear programming and semidefinite programming using interior-points methods, Mathematical Programming 90(2), pp. 229-261.

[183] Toh, K. C., Todd, M. J. and Tütüncü, R. H. (1996). SDPT3 - a matlab software package for semidefinite programming, Technical report, School of Operations Research and Industrial Engineering, Cornell University.

[184] Vostrikova, L. Yu. (1983). Functional limit theorems for the disorder problem. Stochastics, Vol. 9, N. 1-2, pp. 103-104.

[185] Vostrikova, L. Yu. (1987). Functional limit theorems for the likelihood ratio processes. Ann. Universitatis Scientarum Budapestisnenses de R. Eötvös nominatae, VI pp. 145-182.

[186] Vostrikova, L. Yu. (1988). On weak convergence of likelihood ratio processes of general statistical parametric models. Stochastics 23, pp. 277-298

[187] Vostrikova, L. Yu. (1991). Divergence processes and weak convergence of likelihood ratio processes. Séminaire de Probabilités de Rennes I, pp. 134-146.

[188] Winkel, M. (2001). The recovery problem for time-changed Lévy processes. MaPhySto Research Report.

[189] Wong, E. and Zakai, M. (1965) On the relationship between ordinary and stochastic differential equations. Int. J. Eng. Science 3, pp. 213-229.

[190] Wong, E. and Zakai, M. (1965) On the convergence of ordinary integrals to stochastic integrals. Ann. Math. Stat. 36. pp. 1560-1564.

[191] Zhou, C. (1997). A jump-diffusion approach to modeling credit risk and valuing defaultable securities. Working paper, Federal Reserve Board, Washington DC. 